\title{
Measurement of Inclusive Radiative B-Meson Decay B -> X_s gamma
}

\author{
V. E. Ozcan \\ Stanford Linear Accelerator Center \\ Stanford University \\ Stanford, CA 94309
}

SLAC-Report-795

Prepared for the Department of Energy under contract number DE-AC02-76SF00515

Printed in the United States of America. Available from the National Technical Information Service, U.S. Department of Commerce, 5285 Port Royal Road, Springfield, VA 22161. 
This document, and the material and data contained therein, was developed under sponsorship of the United States Government. Neither the United States nor the Department of Energy, nor the Leland Stanford Junior University, nor their employees, nor their respective contractors, subcontractors, or their employees, makes an warranty, express or implied, or assumes any liability of responsibility for accuracy, completeness or usefulness of any information, apparatus, product or process disclosed, or represents that its use will not infringe privately owned rights. Mention of any product, its manufacturer, or suppliers shall not, nor is it intended to, imply approval, disapproval, or fitness of any particular use. A royalty-free, nonexclusive right to use and disseminate same of whatsoever, is expressly reserved to the United States and the University. 


\title{
MEASUREMENT OF INCLUSIVE RADIATIVE $B$-MESON DECAY $B \rightarrow X_{s} \gamma$
}

\author{
A DISSERTATION \\ SUBMITTED TO THE DEPARTMENT OF APPLIED PHYSICS \\ AND THE COMMITTEE ON GRADUATE STUDIES \\ OF STANFORD UNIVERSITY \\ IN PARTIAL FULFILLMENT OF THE REQUIREMENTS \\ FOR THE DEGREE OF \\ DOCTOR OF PHILOSOPHY
}

Veysi Erkcan Özcan

December 2005 
(c) Copyright by Veysi Erkcan Özcan 2006 All Rights Reserved 
I certify that I have read this dissertation and that, in my opinion, it is fully adequate in scope and quality as a dissertation for the degree of Doctor of Philosophy.

\section{Vera Lüth Principal Co-Advisor}

I certify that I have read this dissertation and that, in my opinion, it is fully adequate in scope and quality as a dissertation for the degree of Doctor of Philosophy.

\section{Robert Siemann}

(SLAC and Department of Applied Physics) Principal Co-Advisor

I certify that I have read this dissertation and that, in my opinion, it is fully adequate in scope and quality as a dissertation for the degree of Doctor of Philosophy.

Joanne L. Hewett

(SLAC) 
I certify that I have read this dissertation and that, in my opinion, it is fully adequate in scope and quality as a dissertation for the degree of Doctor of Philosophy.

David W.G.S. Leith

(SLAC)

Approved for the University Committee on Graduate Studies. 


\section{Foreword}

Radiative decays of the $B$ meson, $B \rightarrow X_{s} \gamma$, proceed via virtual flavor changing neutral current processes that are sensitive to contributions from high mass scales, either within the Standard Model of electroweak interactions or beyond. In the Standard Model, these transitions are sensitive to the weak interactions of the top quark, and relatively robust predictions of the inclusive decay rate exist. Significant deviation from these predictions could be interpreted as indications for processes not included in the minimal Standard Model, like interactions of charged Higgs or SUSY particles.

The analysis of the inclusive photon spectrum from $B \rightarrow X_{s} \gamma$ decays is rather challenging due to high backgrounds from photons emitted in the decay of mesons in $B$ decays as well as $e^{+} e^{-}$annihilation to low mass quark and lepton pairs. Based on 88.5 million $B \bar{B}$ events collected by the BABAR detector, the photon spectrum above $1.9 \mathrm{GeV}$ is presented. By comparison of the first and second moments of the photon spectrum with QCD predictions (calculated in the kinetic scheme), QCD parameters describing the bound state of the $b$ quark in the $B$ meson are extracted:

$$
m_{b}=(4.45 \pm 0.16) \mathrm{GeV} / c^{2} \quad \mu_{\pi}^{2}=(0.65 \pm 0.29) \mathrm{GeV}^{2}
$$

These parameters are useful input to non-perturbative QCD corrections to the semileptonic $B$ decay rate and the determination of the CKM parameter $\left|V_{u b}\right|$.

Based on these parameters and heavy quark expansion, the full branching fraction is obtained as:

$$
\mathcal{B}\left(B \rightarrow X_{s} \gamma\right)^{E_{\gamma}>1.6 \mathrm{GeV}}=(4.05 \pm 0.32(\text { stat }) \pm 0.38(\text { syst }) \pm 0.29(\text { model })) \times 10^{-4}
$$


This result is in good agreement with previous measurements, the statistical and systematic errors are comparable. It is also in good agreement with the theoretical Standard Model predictions, and thus within the present errors there is no indication of any interactions not accounted for in the Standard Model. This finding implies strong constraints on physics beyond the Standard Model. 


\section{Acknowledgements}

There are many [ialope without whom this thesis would not be here today. However, there are two people I must mention first, because without them I would not even dream about taking the long journey of which this thesis is just a cobblestone. My late grandfather, Rahmi Tunçağıl and my uncle Yılmaz Bektaşer were the ones who encouraged my interests in science as a little kid. They treated a 10-year-old boy as an adult and discussed with him science, philosophy and theology. When that boy started writing his first thesis, they took him seriously; I remember my grandfather telling me it should even have an acknowledgements page. And now, this second thesis of that boy is dedicated to these two individials.

I do not know how to thank my advisors Vera Lüth and Colin Jessop. Throughout my graduate career, they have always been helpful and patient. I vividly remember my first day at SLAC, talking to Colin: his enthusiasm for doing things together then and all throughout the past six years. Another moment I remember as vividly is Christmas 2005 when Vera had invited me to her family gathering, I had felt as a part of her family that day. Anyone who has the chance of working closely with her feels this sense of extended family feeling; Vera really tries to help you achieve the best for you. She is so caring, patient and understanding, and at the same time constructively critical; without her this thesis would not possible.

I would like to thank each member of my research group, with whom I have worked closely in the last four years for the analysis presented here, Francesca Bucci, Alan Eisner, Colin Jessop, James Libby, Ruth Schmitz, Jane Tinslay and John Walsh. I learned so many things working together with them. I learned how to be meticulous without loosing sight of the big picture, how to be patient, but also ready to move 
on. It has been a wonderful experience to work with them. I must mention that Francesca and Ruth are also very close friends with whom we have shared much more than work.

My years at Stanford would be empty without my friends. Thanks to all members of Stanford Arkadash and Yore folk dance groups, and my friends from the Turkish Student Association at Stanford, I always felt as if I was back home in Turkey. I must thank Serkan Apaydın, Utkan Demirci, Ali Özer Ercan, Onur Fidaner and Nesrin Özalp in particular. Among many other things at Stanford, I will miss the monday-night dancing in Leslie Gordon's classes.

Similarly SLAC would not be the same without the wonderful people there. All members of group $\mathrm{C}$ deserve many thanks, in particular Rainer Bartoldus, Philip Bechtle, Jochen Dingfelder, Mandeep Gill, Amanda Weinstein and my past and present officemates Mark Allen, Aron Soha, Hiro Tanaka and Josh Thompson. Outside of group C, friends Melissa Berry, Hojeong Kim, and my adopted family Aslı, Belis, Mark and Tülay Dönszelmann deserve my thanks for their support and for the fun. Additionally, I must mention that I am grateful to our administrative assistants, Lilian DePorcel, Judy Meo, Mika Stratton and Anna Pacheco at SLAC, and Claire Nicholas and Paula Perron at Department of Applied Physics, for covering me up everytime I was late in returning some sort of paperwork. Similarly my thesis reading committee, JoAnne Hewett, David Leith and Robert Siemann were extremely accomodating although I was quite late in turning in my final draft. I feel grateful for their useful comments and encouragement.

Finally, the largest thanks go to my friends Nicolas Berger, Tetiana Hryn'ova, Murat Korkmaz, Jörg Stelzer, Kartal Toker and Erhan Yenilmez. I am grateful to them for making my life at Stanford meaningful. Similar thanks to my family in Turkey, and my patient wife Feyza, for all the support they provided and the unconditional love I felt constantly regardless of the two continents and an ocean lying in between Stanford and Ankara. 


\section{Contents}

Foreword v v

Acknowledgements vii

1 Theorv and Experimental Challenge 1

1.1 The Standard Model . . . . . . . . . . . . . . . . . . 1

1.2 Electroweak Interactions $\ldots \ldots \ldots \ldots \ldots$

1.3 Quantum Chromodvnamics . . . . . . . . . . . . . . . . 3

$1.4 \quad B \rightarrow X_{\mathrm{s}} \gamma$ Branching Fraction $\ldots \ldots \ldots \ldots \ldots$

1.5 Supersvmmetrv ........................ 6

1.6 Heavv Quark Expansion . . . . . . . . . . . . . . . . 7

$1.7 \quad B \rightarrow X_{8} \gamma$ Photon Spectrum $\ldots \ldots \ldots \ldots$

1.8 Signal Models . . . . . . . . . . . . . . . . . . . . . . . . . 12

1.9 Experimental Techniques . . . . . . . . . . . . . . . . . . 15

2 The BABAR Experiment 20

2.1 The PEP-II $B$ Factory $\ldots \ldots \ldots \ldots \ldots \ldots \ldots$

2.2 The BABAR Tracking Svstem . . . . . . . . . . . . . . . . . . . . . 22

2.2.1 The BABAR Magnet Svstem . . . . . . . . . . . . . . 23

2.2 .2 Silicon Vertex Tracker . . . . . . . . . . . . . . . . . 23

2.2 .3 Drift Chamber . . . . . . . . . . . . . . . . 26

2.2.4 Overall Performance of the Tracking Svstem . . . . . . . . 28

2.3 Ring-Imaging Cherenkov Detector . . . . . . . . . . . . . . . . . . . . . . 29

2.4 Electromagnetic Calorimeter . . . . . . . . . . . . . . . . . . . . . 31 
2.5 Instrumented Flux Return $\ldots \ldots \ldots \ldots \ldots$

2.6 The BABAR Triggen . . . . . . . . . . . . . . . . . . . . . 37

2.6 .1 Level 1 Trigger Svstem . . . . . . . . . . . . . . . . . 37

2.6.2 Level 3 Trigger Svstem . . . . . . . . . . . . . . . . . . 38

2.7 Data Sets and Simulation $\ldots \ldots \ldots \ldots$

3 Event Selection $\quad 42$

3.1 Selection of High-Quality Particle Candidates $\ldots . . . . . . .443$

3.2 Event Preselection . . . . . . . . . . . . . . . . . . . 43

3.3 Selection of the Signal Photon . . . . . . . . . . . . . 44

3.3.1 Photon Qualitv Criteria . . . . . . . . . . . . . . . 44

3.3.2 Shower Shape and Isolation Requirements . . . . . . . . . . . 49

$3.3 .3 \pi^{0}$ and $n$ Vetoes . . . . . . . . . . . . . . . . . 51

3.4 Selection of the Photon Energy Range . . . . . . . . . . . 52

3.5 Event Topologv . . . . . . . . . . . . . . . . . . . . . . . . 54

3.6 Removal of Multiple-Candidate Events . . . . . . . . . . . . . 58

3.7 Lepton Tagging . . . . . . . . . . . . . . . . . . . . . . . . . . 58

3.7 .1 Lepton Identification . . . . . . . . . . . . . . . . . . . . 59

3.7.2 Kinematic Requirements on Lepton Candidates . . . . . . . 61

3.8 Optimization of the Selection Criteria . . . . . . . . . . . . 65

3.9 Requirement on Effective Multiplicity . . . . . . . . . . . . . . 67

3.10 Efficiency vs. Photon Energv . . . . . . . . . . . . . . . . . 68

3.11 Expectated Signal and Backgrounds after Selection . . . . . . . . 75

$\begin{array}{lll}4 & B \bar{B} \text { Background } & \mathbf{7 8}\end{array}$

4.1 Components of the $B \bar{B}$ Backgrounds . . . . . . . . . . . . 78

4.2 Backoround from $\pi^{0}$ and $n$ Decavs . . . . . . . . . . . . . 80

4.2.1 Selecting Samples of Inclusive $\pi^{0}(\eta)$ Events . . . . . . . . . . 80

4.2.2 Yields from $\pi^{0}$ Mass Distributions . . . . . . . . . . . . . . 81

4.2 .3 Yields from $n$ Mass Distributions _... . . . . . . . . 86

4.2.4 Energy Spectra and Correction Factors ... . . . . . . . . . . 91

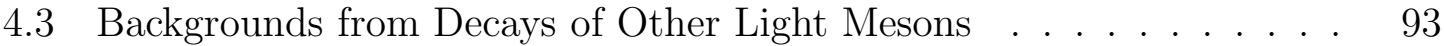


4.4 Background from Electrons . . . . . . . . . . . . . . . . . 94

4.4.1 Electron Inefficiencv in Bhabha Events . . . . . . . . . . . 95

4.4.2 Inefficiencv due to Multihadronic Environment . . . . . . . . 96

4.4.3 Corrections to Electrons in $B \bar{B}$ Simulation . . . . . . . . . 99

4.5 Hadronic Component of the Background . . . . . . . . . . . . . 101

4.5 .1 Antinucleon Production . . . . . . . . . . . . . . . 102

4.5.2 Studies of Antinucleon Energv Deposition . . . . . . . . . 105

4.5.3 Studies of Shower Shape Variables. . . . . . . . . . . . . . 107

4.5.4 Corrections to the Antinucleons in $B \bar{B}$ Simulation . . . . . . 109

4.6 Final-State Radiation from Light Ouarks . . . . . . . . . . . . . . . 109

4.7 Overall Corrections to $B \bar{B}$ Simulation . . . . . . . . . . . . . . . 112

5 Photon Spectrum 118

5.1 Scaling of Photon $E^{*}$ in Off-resonance Data . . . . . . . . . 118

5.2 Data in Control Regions . . . . . . . . . . . . . . . . . . . 119

5.3 Signal Yields . . . . . . . . . . . . . . . . . . . . . . 120

6 Svstematic Corrections and Uncertainties 123

6.1 Photon Selection . . . . . . . . . . . . . . . . . . . . . . 124

6.1.1 Shower Identification and Selection . . . . . . . . . . . . 124

$6.1 .2 \pi^{0}$ and $n$ Vetoes . . . . . . . . . . . . . . . . 127

6.2 Event Topologv Selection . . . . . . . . . . . . . . . . . . . . 128

6.2.1 Fisher Discriminant in Data and Simulation . . . . . . . . . 129

6.2.2 Altering the Fragmentation Model . . . . . . . . . . . . . . 133

6.3 Lepton Tagoing . . . . . . . . . . . . . . . . . . . . . . 136

6.3.1 Corrections to Lepton Identification _ . . . . . . . . . . 137

6.3.2 Corrections on the Semileptonic B Decavs with Charm . . . . 138

6.3 .3 Other Sources of Tag Leptons . . . . . . . . . . . . . . . 140

6.3.4 Lepton Corrections on Subtracted $B \bar{B}$ Background . . . . . . 141

6.4 Data Sample Normalization . . . . . . . . . . . . . . . . . . . 142

6.5 Simulation Based $B \bar{B}$ Subtraction . . . . . . . . . . . . . . . . 143

6.6 Signal Monte Carlo Simulation . . . . . . . . . . . . . . . . . . . . 144 
$\begin{array}{lll}7 & \text { Results } & 146\end{array}$

7.1 Model Dependence of Selection Efficiencv . . . . . . . . . . . . . 146

7.2 Partial Branching Fractions in $\Upsilon(4 S)$ Frame . . . . . . . . . . . 151

7.3 Truncated Moments in the B-Meson Rest Frame . . . . . . . . . . . . 153

7.4 Correlations . . . . . . . . . . . . . . . . . . . . . . . 156

7.5 Extraction of HQE Parameters $\ldots \ldots \ldots \ldots \ldots$

8 Interpretation of Results 161

8.1 Photon Spectrum . . . . . . . . . . . . . . . . . . . . 161

8.2 Moments of the Photon Spectrum . . . . . . . . . . . . . . . . 161

8.3 Total Branching Fraction . . . . . . . . . . . . . . . . . . . . . 164

8.4 Future Prospects . . . . . . . . . . . . . . . . . . . . . . . 164

$\begin{array}{ll}\text { A Signal Model Parameters } & 167\end{array}$

A.1 The Kinetic Scheme. . . . . . . . . . . . . . . . . . 167

A.2 The Shape Function Scheme . . . . . . . . . . . . . . . . . . . 169

\begin{tabular}{ll}
\hline B VCS Samples & 171
\end{tabular}

$\begin{array}{ll}\text { Bibliography } & 173\end{array}$ 


\section{List of Tables}

$1.1 K^{*}(892) \gamma$ fraction for Kagan-Neubert signal model $\ldots \ldots \ldots \ldots . . . \quad 15$

2.1 Monte Carlo and real data sets $\ldots \ldots \ldots \ldots . \ldots . \ldots 40$

3.1 Good track and good photon identification criterial . . . . . . . . . 43

3.2 Effiencies of selection criteria for $B \rightarrow X_{8} \gamma$ and $B \rightarrow K^{*} \gamma \ldots \ldots$

3.3 Effiencies of selection criteria for $q \bar{q}$ continuum background . . . . . . 46

3.4 Effiencies of selection criteria for $\tau^{+} \tau^{-}$continuum background . . . 47

3.5 Effiencies of selection criteria for $B \bar{B}$ background $\ldots \ldots \ldots . . \ldots 48$

3.6 Energv dependent $\pi^{0}$ and $n$ vetoes. . . . . . . . . . . . . 53

3.7 Fisher discriminant parameters $\ldots \ldots \ldots \ldots \ldots \ldots$

3.8 Efficiencies of possible $N_{\text {mult }}$ requirements vs. $X_{\text {c signal multiplicity }}$. 69

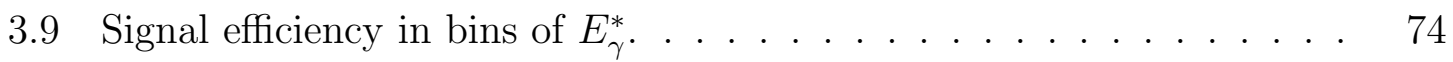

$3.10 \mathrm{MC}$ expectations for signal and backgrounds after selection criteria . 77

4.1 Breakdown of $\mathrm{MC} B \bar{B}$ backgrounds by photon origin . . . . . . $\quad 79$

4.2 Selection of $\pi^{0}$ and $\eta$ candidates for $B \bar{B}$ background corrections . . . 81

$4.3 \pi^{0}$ mass fit validation $\ldots \ldots \ldots \ldots \ldots$

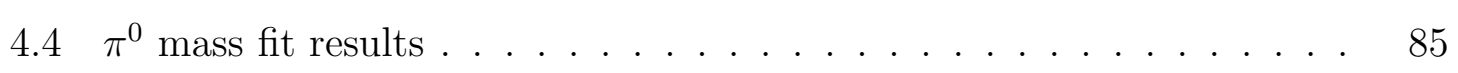

$4.5 \pi^{0}$ mass fit validation for untagged $B \bar{B}$ Monte Carlo events . . . . . . 85

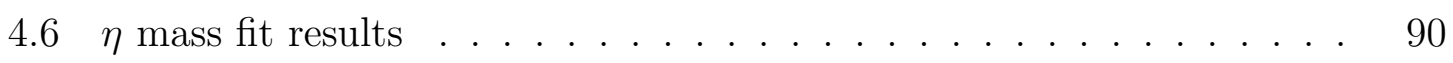

$4.7 \eta$ mass fit validation for untagged $B \bar{B}$ Monte Carlo events . . . . . 90

$4.8 \pi^{0}$ correction factors to $B \bar{B}$ simulation $\ldots \ldots \ldots \ldots \ldots . \ldots . \ldots 92$

$4.9 \eta$ correction factors to $B \bar{B}$ simulation $\ldots \ldots \ldots \ldots \ldots \ldots \ldots$ 


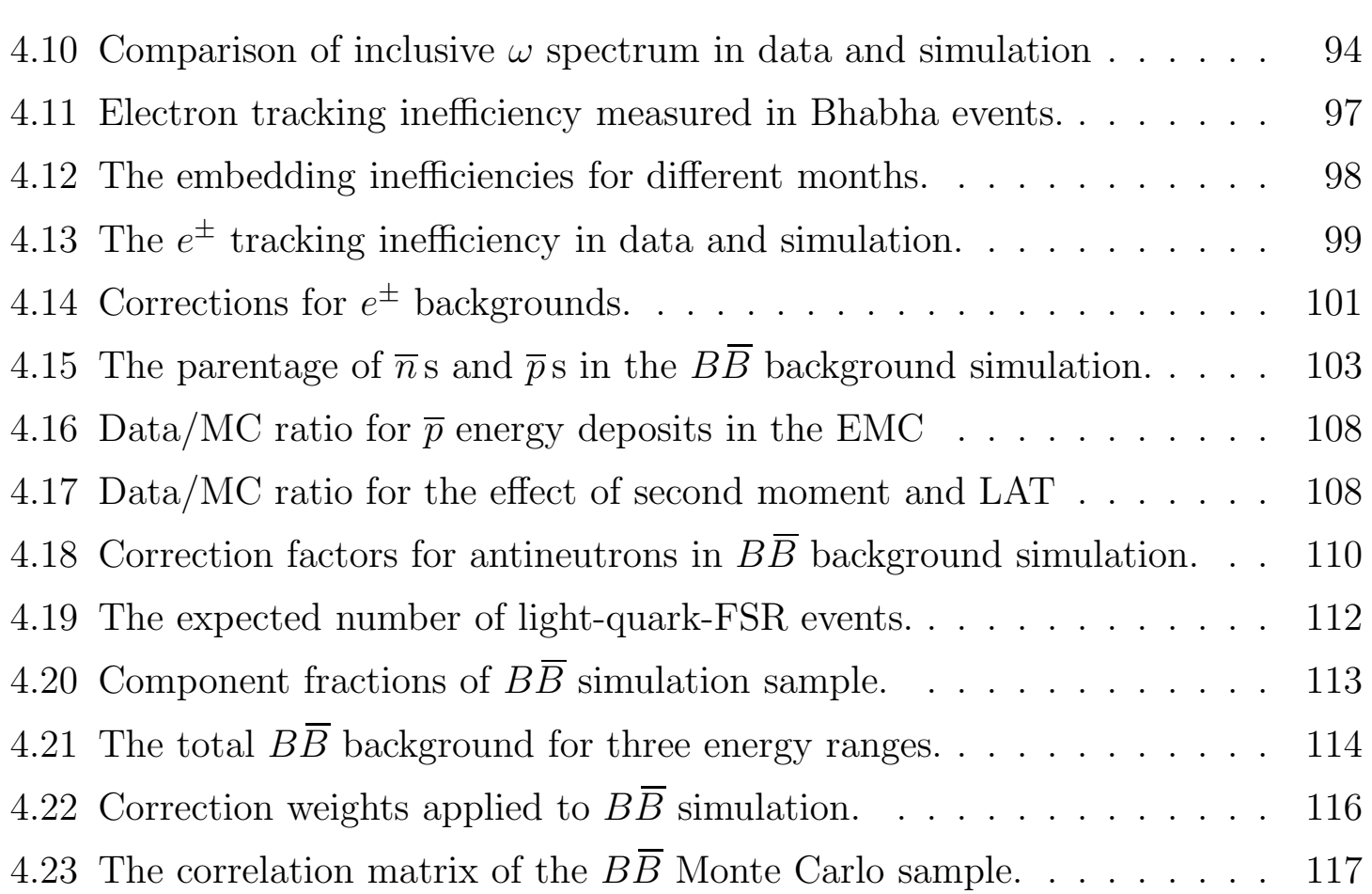

5.1 The backoround estimation compared to data in the control regions. 119

5.2 Unblinded data and signal vields after background subtraction . . . . 121

5.3 Extracted signal vields in ranges of $E^{*}$. . . . . . . . . . . . . . . 122

6.1 Svstematic uncertainties ........................... 124

6.2 Svstematic uncertainties associated with photon selection . . . . . . . 125

6.3 Measured ratio of low-energy photons, data to simulation . . . . . . . 127

6.4 Dependence of signal efficiency on multiplicitv bv energv bin . . . . . 134

6.5 Dependence of efficiencv on $X_{\odot} n_{\pi_{0}} / n_{\pi^{ \pm}}$ratio for MC simulation. . . 135

6.6 Corrections to semileptonic $B$ decavs . . . . . . . . . . . . . . . . . . 139

6.7 Svstematic uncertainties on the $B \bar{B}$ background estimate. . . . . . . 143

7.1 Efficiencies for alternative signal models as compared to default . . . 147

7.2 Mean value of the true $E^{*}$ in bins of reconstructed $E^{*}$. . . . . . . . . 148

7.3 Mean $E_{*}^{*}$ vs. selection efficiency for different signal models . . . . . . 149

7.4 Partial branching fractions . . . . . . . . . . . . . . . . 151

7.5 Example derivation of the $\Delta_{c u t}$ correction to $E_{*}^{*}$ mean . . . . . . . . 154 
7.6 First moments of the measured photon spectrum . . . . . . . . . . 155

7.7 Centralized second moments of the measured photon spectrum . . . . 155

7.8 Correlation matrix for statistical errors on measured moments . . . 157

7.9 Correlation matrix for svstematic errors on measured moments . . . 158

7.10 Correlation matrix for model-dependence errors on measured moments 159

8.1 Moments of the measured photon spectrum . . . . . . . . . 162

8.2 Comparison of measured branching fraction with other experiments. . 165

A.1 Parameters of models based on Benson-Bigi-Uraltsev calculations . . 168

A.2 Parameters of models based on LNP calculations . . . . . . . . 170 


\section{List of Figures}

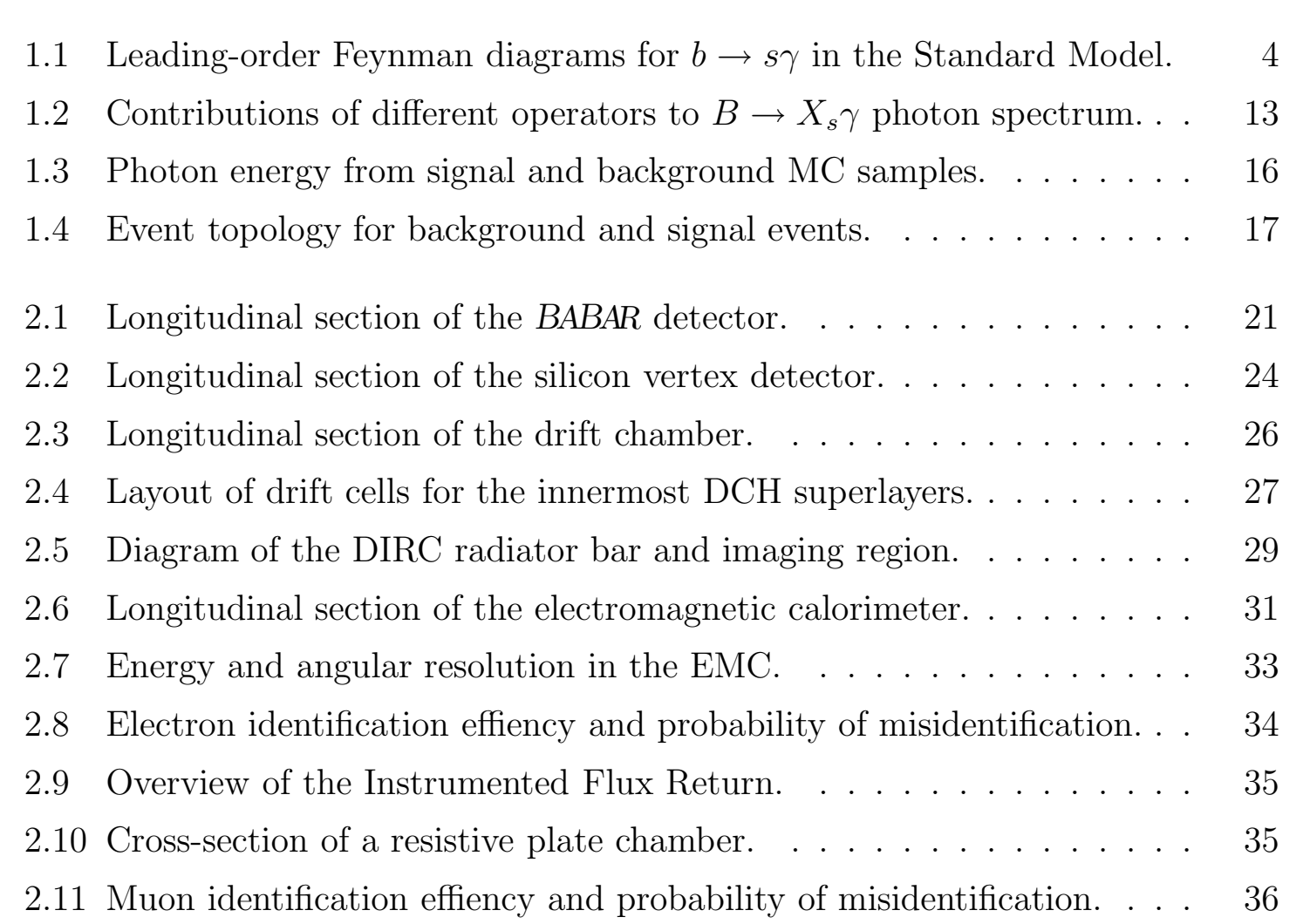

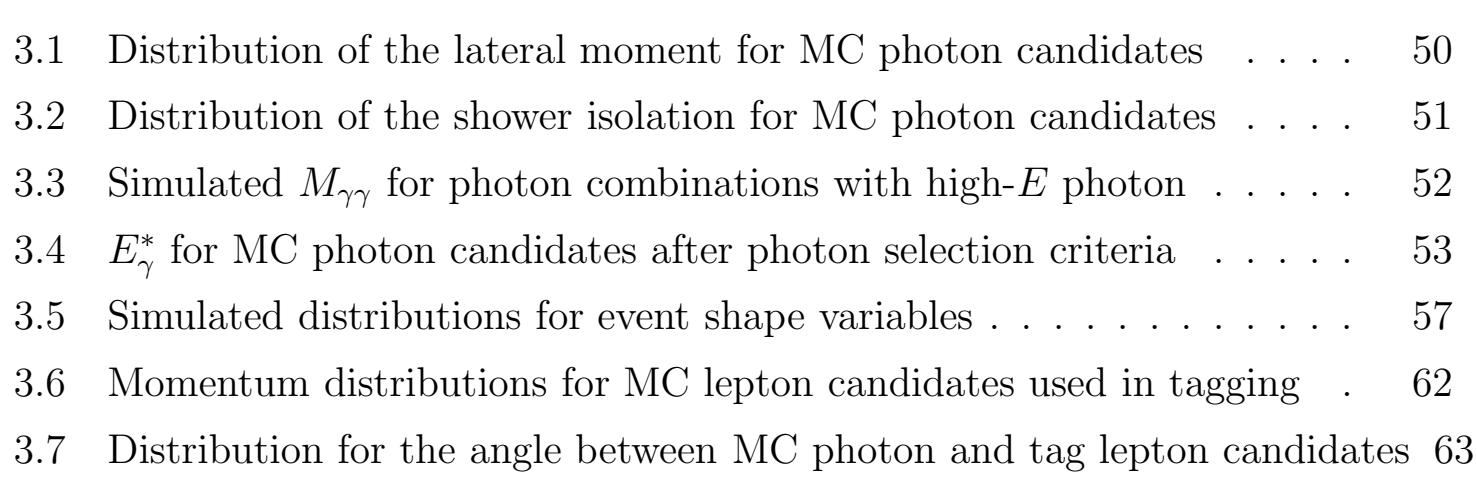


3.8 Missing energy distributions for tagged events from MC simulation . 65

3.9 Sample optimization plots for electron tagging criterial . . . . . . . 66

3.10 Acceptance component of signal efficiencv vs. true $E^{*} \ldots \ldots \ldots . .70$

3.11 True vs. Reconstructed $E_{*}^{*}$ for KN480 signal MC after acceptance . . 71

3.12 Overall selection efficiencv for signal vs. reconstructed $E^{*} \ldots \ldots \ldots$. . . . 72

$3.13 \mathrm{KN} 480$ model overall efficiencv for signal vs. reconstructed $E_{*}^{*} \ldots \ldots 73$

$3.14 E^{*}$ spectrum for MC signal and backgrounds after all selection criteria 76

4.1 Mass fits to Monte Carlo truth-matched candidates . . . . . . . . 84

$4.2 \pi^{0}$ mass fits in simulated $B \bar{B}$ events . . . . . . . . . . . . 87

$4.3 \pi^{0}$ mass fits in on-resonance data events $\ldots \ldots \ldots \ldots$

$4.4 \pi^{0}$ mass fits in off-resonance data events . . . . . . . . . . . 89

$4.5 \pi^{0}$ and $\eta$ c.m.-energv spectra in data and $B \bar{B}$ simulation . . . . . . 91

4.6 Data/MC ratio for $p / \bar{p}$ inclusive cross section from $\Upsilon(4 S) \ldots \ldots$

4.7 The c.m.momentum of antinucleons in the $B \bar{B}$ simulation. . . . . . 104

4.8 Calorimeter response to $\bar{p}$ interactions in data and simulation . . . . 106

4.9 Shower energy divided by total available energy in $\bar{p}$ interactions . . . 107

4.10 The theoretical light quark FSR spectrum . . . . . . . . . . . 111

5.1 Photon spectrum before efficiency corrections. . . . . . . . . . . . 122

6.1 Fisher discriminant distributions in the $\pi^{0}$ control sample. . . . . . . 131

6.2 Fisher discriminant distributions in the continuum. . . . . . . . . 132

6.3 Signal efficiencr vs. possible shifts in Fisher discriminant output. . . 133

6.4 Svstematic change in selection efficiencv vs. PID efficiencies. . . . . 137

7.1 Efficiencv vs. mean reconstructed energy for signal models . . . . . . 149

7.2 Efficiencv-corrected $E_{*}^{*}$ spectrum. . . . . . . . . . . . . . 152

7.3 HQE parameters from fits to the moments in the kinetic scheme .. . 160

8.1 Comparison of measured moments with other experiments. . . . . 163

8.2 Comparison of measured branching fraction with other experiments. . 165

B.1 Energy and polar angle distributions for VCS photons. . . . . . . 172 
xviii 


\section{Chapter 1}

\section{Theory and Experimental Challenge}

\subsection{The Standard Model}

The Standard Model of particle physics is a quantum field theory in four-dimensional Minkowski space that provides an elegant framework to understand and make precise predictions about three of the four fundamental interactions of nature. Tested extensively in the last three decades, it has been shown to be the most successful theory of matter up to the energy scales that experiments have been able to probe. Despite its triumphs however, it is considered to be the effective form of a more fundamental theory, due to both aesthetic reasons, such as the fact that it depends on 18 a priori arbitrary parameters [1], and practical deficiencies, such as the absence of neutrino oscillations.

The fundamental constituents of matter in the Standard Model are spin- $\frac{1}{2}$ fermions, which interact through the exchange of spin-1 gauge bosons. The fermions are categorized into two broad groups, depending on whether they carry color charges or not: quarks $\left(u_{i}=u, c, t\right.$ and $\left.d_{i}=d, s, b\right)$ and leptons $\left(\nu_{i}=\nu_{e}, \nu_{\mu}, \nu_{\tau}\right.$ and $\left.\ell_{i}=e^{-}, \mu^{-}, \tau^{-}\right)$. Hence quarks interact with the carriers of the color (strong) force, gluons. While only quarks and charged leptons have electric charge, and thus couple to electromagneticforce-carrier photons, all fermions including neutrinos have weak charge and therefore 
couple with $W^{ \pm}$and $Z^{0}$ bosons.

\section{$1.2 \quad$ Electroweak Interactions}

In the Standard Model, electromagnetic and weak interactions are governed by a unified chiral gauge theory of gauge group $S U(2)_{L} \times U(1)_{Y}$. While right-handed fermion fields are $\mathrm{SU}(2)$ singlets, left-handed fermions of $i^{\text {th }}$ generation are represented by $\mathrm{SU}(2)$ doublets, $\psi_{i}=\left(\begin{array}{c}\nu_{i} \\ \ell_{i}^{-}\end{array}\right)$or $\left(\begin{array}{c}u_{i} \\ d_{i}^{\prime}\end{array}\right)$, where $d_{i}^{\prime} \equiv \sum_{j} V_{i j} d_{j}$. Here $V_{i j}$ is a $3 \times 3$ unitary matrix known as the Cabibbo-Kobayashi-Maskawa matrix [4].

There are three vector fields, $W^{i}, i=1,2,3$, associated with the $S U(2)_{L}$ symmetry and another, $B$, associated with $U(1)$. The corresponding coupling constants are denoted by $g$ and $g^{\prime}$. With the introduction of a complex scalar doublet, named the Higgs field, the $S U(2)_{L} \times U(1)_{Y}$ symmetry is spontaneously broken into $U(1)_{e m}$ symmetry of the electromagnetic interaction. Three degrees of freedom from the Higgs scalar are absorbed by the gauge bosons which acquire mass and become the neutral and charged gauge bosons of the weak interaction: $Z \equiv-B \sin \theta_{W}+W^{3} \cos \theta_{W}$ and $W^{ \pm} \equiv \frac{1}{\sqrt{2}}\left(W^{1} \mp i W^{2}\right)$. The field $A \equiv B \cos \theta_{W}+W^{3} \sin \theta_{W}$ remains massless and is identified as the photon, where $\theta_{W} \equiv \tan ^{-1}\left(g^{\prime} / g\right)$ is known as the weak mixing angle. The Lagrangian, after the mentioned symmetry breaking, is given by:

$$
\begin{aligned}
\mathcal{L}_{E W} & =\sum_{i} \bar{\psi}_{i}\left(i \gamma^{\mu} \partial_{\mu}-m_{i}-\frac{g m_{i} H}{2 M_{W}}\right) \psi_{i} \\
& -\frac{g}{2 \sqrt{2}} \sum_{i} \bar{\psi}_{i} \gamma^{\mu}\left(1-\gamma^{5}\right)\left(T^{+} W_{\mu}^{+}+T^{-} W_{\mu}^{-}\right) \psi_{i} \\
& -e \sum_{i} \mathrm{q}_{i} \bar{\psi}_{i} \gamma^{\mu} \psi_{i} A_{\mu}-\frac{g}{2 \cos \theta_{W}} \sum_{i} \bar{\psi}_{i} \gamma^{\mu}\left(g_{V}^{i}-g_{A}^{i} \gamma^{5}\right) \psi Z_{\mu},
\end{aligned}
$$

where $q_{i}$ is the electric charge of $\psi_{i}$ in units of the positron charge $e=g \sin \theta_{W}, T^{+}$and $T^{-}$are the weak isospin raising and lowering operators, and $g_{V}^{i} \equiv t_{3, i}-2 \mathrm{q}_{\mathrm{i}} \sin ^{2}\left(\theta_{\mathrm{W}}\right)$ and $g_{A}^{i} \equiv t_{3, i}$ are the vector and axial weak couplings of the fermions to the $Z^{0}$ boson. $t_{3}$, the third component of the weak isospin, is $t_{3}=+\frac{1}{2}$ for the up-type quarks $\left(u_{i}\right)$ and neutrinos $\left(\nu_{i}\right)$. For the down-type quarks $\left(d_{i}\right)$ and charged leptons $\left(\ell_{i}\right), t_{3}=-\frac{1}{2}$. 


\subsection{Quantum Chromodynamics}

Quantum chromodynamics (QCD) is the non-Abelian gauge theory of the strong interaction in the Standard Model. Its Lagrangian is given by (up to gauge-fixing terms):

$$
\begin{aligned}
\mathcal{L}_{Q C D} & =\sum_{q} \bar{q}\left(i \gamma^{\mu} D_{\mu}-m_{q}\right) q-\frac{1}{4} G_{a}^{\mu \nu} G_{a \mu \nu}, \\
G_{a}^{\mu \nu} & =\partial^{\mu} G_{a}^{\nu}-\partial^{\nu} G_{a}^{\mu}-g_{s} f_{a b c} G_{b}^{\mu} G_{c}^{\nu}
\end{aligned}
$$

where $g_{s}$ is the coupling constant, and $D_{\mu}=\partial_{\mu}+i g_{s} T_{a} G^{a}$ is the covariant derivative with $T_{a}$ the generators and $f_{a b c}$ the structure constants of the $S U(3)$ algebra. Each quark field of a specific flavor, $q(x)$ is represented by a color-triplet of Dirac spinors, and the $G_{a}^{\mu}(x)$ are the eight massless Yang-Mills (gluon) fields. The quark mass terms are a result of the Yukawa couplings to the Higgs field.

The running of the effective QCD coupling $\alpha_{S}=g_{s}^{2} / 4 \pi$ is governed by a negativedefinite $\beta$-function and thus implies an interesting feature of QCD: asymptotic freedom. At short distance scales, QCD vacuum is effectively screening the color charge, allowing quarks to behave as free particles. In this regime, QCD can be treated perturbatively.

On the other hand, QCD has a very different characteristic at long distances. In this soft regime, confinement binds quarks always into color-neutral hadrons; no free quarks have ever been observed. The dynamics of these soft degrees of freedom is non-perturbative, making precise quantitative predictions difficult and requiring various approximation techniques such as the $1 / N$ expansion, chiral perturbation theory, lattice calculations. Fortunately, the lowest order coefficient in the $\beta$-function is numerically large, so that even for as low a normalization scale as $\mu \sim 1 \mathrm{GeV}$, the coupling constant is small enough to let perturbative effects be decoupled in this non-perturbative soft regime. 


\section{$1.4 \quad B \rightarrow X_{s} \gamma$ Branching Fraction}

Flavor change in the Standard Model is possible only in weak decays involving a $W^{ \pm}$ boson; flavor-changing neutral currents (FCNCs) are absent at the tree level. This makes the transitions such as $b \rightarrow s \gamma$, which must proceed through loop diagrams (Figure 1.1), particularly sensitive to physics effects beyond the Standard Model. Since the early 1980s, when its rate was studied as a function of then-to-be-discovered top-quark mass [2], this rare decay has been an essential tool for the phenomologists, studied over and over, constraining a multitude of different fundamental models [3].
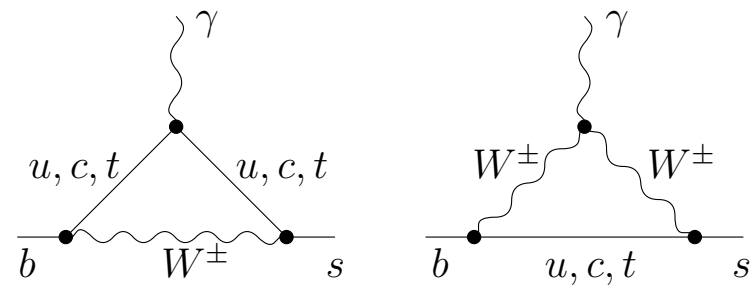

Figure 1.1: Leading-order Feynman diagrams, penguin loops, for $b \rightarrow s \gamma$ transition in the Standard Model.

At the hadron level, $b \rightarrow s \gamma$ is the underlying process for many decays such as $B \rightarrow K^{*}(892) \gamma, B_{s} \rightarrow f \gamma$ or $\Lambda_{b} \rightarrow \Lambda \gamma$. Inclusive decay rates, in which all the final states $X_{s} \gamma$ from the decay of a given hadron are added, can be computed with high precision. As such, the computation of the inclusive $B \rightarrow X_{s} \gamma$ decay rate has been one of the most detailed undertakings in perturbative QCD. This is possible because of the fact that non-perturbative effects, $\Delta^{\text {nonpert. }} \sim \mathcal{O}\left(\Lambda_{Q C D}^{2} / m_{b}^{2}\right)$, are small and under control due to heavy mass expansion techniques (Section 1.6) and quarkhadron duality [5]:

$$
\begin{array}{r}
\Gamma\left(B \rightarrow X_{s} \gamma\right)=\Gamma\left(b \rightarrow X_{s}^{\text {parton }} \gamma\right)+\Delta^{\text {nonpert. }} \\
\Gamma\left(b \rightarrow X_{s}^{\text {parton }} \gamma\right)=\Gamma(b \rightarrow s \gamma)+\Gamma(b \rightarrow s \gamma g)+\cdots
\end{array}
$$

In order to compute the perturbative decay width $\Gamma\left(b \rightarrow X_{s}^{\text {parton }} \gamma\right)$, the suitable framework is an effective low-energy theory with five quarks, obtained by integrating out the heavy particles, i.e. the gauge bosons and the top quark in the Standard Model. The Lagrangian for this effective theory is given by the operator product 
expansion [6]:

$$
\mathcal{L}_{e f f}(b \rightarrow s \gamma)=\mathcal{L}_{Q C D \times Q E D}(u, d, s, c, b)+\frac{4 G_{F}}{\sqrt{2}} V_{t b} V_{t s}^{*} \sum_{i=1}^{8} C_{i}(\mu) \mathcal{O}_{i}(\mu)
$$

where $\mathcal{O}_{i}$ are the local operators that can be considered as the vertices of the effective theory and $C_{i}$ are the Wilson coeffients, which play the role of coupling constants. $G_{F}=\frac{g^{2}}{4 \sqrt{2} M_{W}^{2}}$ is the Fermi constant. The eight operators are given by:

$$
\mathcal{O}_{i}= \begin{cases}\left(\bar{s} \Gamma_{i} c\right)\left(\bar{c} \Gamma_{\underline{\underline{L}}}^{\prime} b\right), & i=1,2 \\ \left(\bar{s} \Gamma_{i} b\right) \sum_{q}\left(\bar{q} \Gamma_{\underline{i}}^{\prime} q\right) & i=3,4,5,6 \quad(q=u, d, s, c, b) \\ \frac{e}{16 \pi^{2}} m_{b} \bar{s} \sigma^{\mu \nu} P_{R} b F_{\mu \nu} & i=7 \\ \frac{g_{s}}{16 \pi^{2}} m_{b} \bar{s} \sigma^{\mu \nu} T^{a} P_{R} b G_{\mu \nu}^{a} & i=8 .\end{cases}
$$

Here, $\Gamma_{i}$ and $\Gamma_{i}^{\prime}$ denote various combinations of the color and Dirac matrices and $P_{R}=\left(1+\gamma_{5}\right) / 2$ [7]. Note that the expansion neglects operators of dimension more than six, since those would be suppressed by higher powers of $1 / m_{W}$.

The computation of the $b \rightarrow X_{s}^{\text {parton }} \gamma$ decay rate is performed in three steps:

Matching. This is the computation of the Wilson coefficients $C_{i}\left(\mu_{0}\right)$ at $\mu_{0} \simeq m_{W}$, also referred to as the initial conditions, by requiring the equality of the Standard Model and effective theory Green functions at external momenta that are much smaller than the masses of the decoupled particles [8].

Mixing. The computed initial conditions are evolved from $\mu_{0}$ down to $\mu=\mu_{b} \sim$ $m_{b}$ using the renormalization group equations, $\mu \frac{d}{d \mu} C_{i}(\mu)=C_{j}(\mu) \gamma_{i j}(\mu)$, where the anomalous dimension matrix $\gamma_{i j}$ is determined from UV divergences in the effective theory [9].

Matrix elements. This step basically includes Bremsstrahlung and virtual corrections. The matrix elements $\left\langle s \gamma\left|\mathcal{O}_{i}(\mu)\right| b\right\rangle$ and $\left\langle s \gamma g\left|\mathcal{O}_{i}(\mu)\right| b\right\rangle$ are calculated at the scale $\mu=\mu_{b}[10]$.

During the past few years, all three steps were completed at the next-to-leading-log (NLL) precision, i.e. resumming all the terms of the form $\alpha_{S}\left(\mu_{b}\right) \alpha_{S}^{n}\left(\mu_{b}\right) \log ^{n}\left(\mu_{b} / \mu_{0}\right)$. After applying electroweak corrections to order $\alpha_{e m}^{\text {on-shell }} \alpha_{S}^{n}\left(\mu_{b}\right) \log ^{n}\left(\mu_{b} / \mu_{0}\right)$ [11] (Here 
$\alpha_{e m}^{\text {on-shell }}=1 / 137$ is the on-shell value of the fine structure constant) and estimating the non-perturbative corrections, the branching fraction is given as [12]:

$$
\mathcal{B}\left(B \rightarrow X_{s} \gamma\right)^{E_{\gamma}>1.6 \mathrm{GeV}}=\left(\left.3.61_{-0.40}^{+0.24}\right|_{\frac{m_{c}}{m_{b}}} \pm 0.02_{\mathrm{CKM}} \pm 0.24_{\text {param. }} \pm 0.14_{\text {scale }}\right) \times 10^{-4}
$$

The largest error is due to the uncertainty in the charm-quark mass. It has recently been shown that this uncertainty could be reduced by about a factor of two by computing the NNLL terms [13]. The second largest uncertainty is due to the uncertainties on the various parameters such as $\alpha_{S}, m_{t}$ etc. Finally, there is a sizeable normalization scale uncertainty, which is estimated by varying the low-energy scale in the range $m_{b} / 2<\mu_{b}<2 m_{b}$.

\subsection{Supersymmetry}

As mentioned earlier, despite its successes, there are reasons to believe that the Standard Model is the low-energy effective form of a more fundamental theory. Towards this end, many extensions have been hypothesized and studied. While some of these extensions are quite ambitious, building on new mathematical frameworks in an attempt to cover gravity as well as the strong and electroweak interactions, others are more modest, trying to fix the perceived deficiencies of the Standard Model, by proposing new broken symmetries within the quantum field theory framework.

Supersymmetry (SUSY) is one such proposed symmetry, considered by many to be the most likely candidate to explain the physics at the $\mathrm{TeV}$ scale. Its appeal is due to the elegant solution it brings to the naturalness problem in the Standard Model. The radiative corrections to the self-energy of fundamental scalar particles, like the Higgs boson, are quadratically divergent. Even if these divergences are renormalized away, there remain additive corrections proportional to the masses of the contributing virtual particles. Apart from the fact that this constitutes a fine-tuning issue, the lack of a custodial symmetry protecting the Higgs mass is unsatisfying.

The solution to this problem in SUSY is to assign superpartners to the Standard Model particles, a boson for each fermion and a fermion for each boson, such that the quadratically-divergent contributions to the Higgs self-energy cancel [14]. Although 
this solution effectively doubles the number of particles, their introduction amazingly provides an answer to another unresolved issue of today's particle physics: gauge coupling unification at the grand-unified-theory scale. Additionally, the lightest among these supersymmetric particles is stable in $R$-parity conserving SUSY models and would thus be a likely candidate for dark matter. Finally, string theories provide solid arguments in favor of SUSY.

SUSY is also very interesting experimentally. While there are alternative solutions to the Higgs naturalness problem, such as Technicolor, which suggests that Higgs or the longitudinal $W$ and $Z^{0}$ bosons might be composite particles [15], most of the simple models based on these solutions have already been ruled out by experiments [4]. On the other hand, SUSY phenomology is very rich and has not been throughly tested yet. SUSY signatures are expected at the LHC experiments [16].

$B \rightarrow X_{s} \gamma$ decay is one of the few measurable processes accessible today for probing the SUSY parameter space in the accelerator environment [17]. If the supersymmetry were not broken, contibutions to $B \rightarrow X_{s} \gamma$ would cancel out and the decay rate would be zero. Sizeable contributions to the decay amplitude are possible with the introduction of charged Higgs bosons replacing the $W^{ \pm}$boson in the SM penguin loop or with new chargino- or gluino-mediated loops [18].

\subsection{Heavy Quark Expansion}

As mentioned briefly in Section 1.3, there are several techniques that are used to calculate QCD in the non-perturbative regime. Heavy quark expansion (HQE) is such a technique constructed to calculate various properties of hadrons containing a single heavy quark, bottom or charm (the top quark, with a $t \rightarrow b+W^{+}$decay width of $\Gamma \approx 1 \mathrm{TeV}$, decays before it can form a hadron). It is particularly useful for studying hadrons with a $b$ quark, since the scale of typical momentum transfers in soft-QCD is of order $\Lambda_{Q C D}$ (few hundred MeV) and thus much smaller than the mass of the $b$ quark, $m_{b} \sim 4.8 \mathrm{GeV} / c^{2}$.

The QCD Lagrangian is formulated at very short distances, i.e. at a very high normalization point $\mu=M_{0} \gg m_{Q}$, where $M_{0}$ is the mass of an ultraviolet regulator, 
and $m_{Q}$ is the mass of a generic heavy quark, $Q$. On the other hand, in order to describe the low-energy properties of a heavy-flavor hadron, $H_{Q}$, a normalization scale $\mu \ll m_{Q}$ is actually more appropriate. At such a scale, the heavy quark can be treated as a non-relativistic quantum-mechanical object.

The well-established technique of separating long and short-distance physics to a construct a low-energy effective theory is the Wilsonean operator product expansion (OPE). An application of this technique has been discussed in Section 1.4 in the context of calculating the partonic decay width $\Gamma\left(b \rightarrow X_{s}^{\text {parton }} \gamma\right)$. In general, the QCD Lagrangian can be evolved down to a desired scale $\mu$, by integrating out the field fluctuations with frequencies above that scale and absorbing them into coefficients $C_{i}(\mu)$. This standard OPE approach can be modified such that the heavy quark field, which appear in the hadron, is not integrated out even though $\mu \ll m_{Q}$.

Neglecting the masses of the light quark fields $(u, d$ and $s)$, Equation 1.2 can be written as the sum of light and heavy contributions:

$$
\mathcal{L}_{Q C D}=-\frac{1}{4} G_{a}^{\mu \nu} G_{a \mu \nu}+\sum_{q=u, d, s} \bar{q} i \gamma^{\mu} D_{\mu} q+\sum_{Q} \bar{Q}\left(i \gamma^{\mu} D_{\mu}-m_{Q}\right) Q
$$

where the last term associated with the heavy quark fields has been explicitly separated out from the other light terms. After the evolution down to the low scale of $\mu$, the form of this Lagrangian changes, and a series of operators of higher dimension appears. The heavy quark part of the Lagrangian takes the form [19]:

$\mathcal{L}_{\text {heavy }}=\sum_{Q}\left\{\bar{Q}\left(i \gamma^{\mu} D_{\mu}-m_{Q}\right) Q+\frac{c_{G}}{2 m_{Q}} \bar{Q} \frac{i}{2} \sigma_{\mu \nu} G_{\mu \nu} Q+\sum_{\Gamma, q} \frac{d_{Q q}^{(\Gamma)}}{m_{Q}^{2}} \bar{Q} \Gamma Q \bar{q} \Gamma q\right\}+\mathcal{O}\left(\frac{1}{m_{Q}^{3}}\right)$

where $c_{G}$ and $d_{Q q}^{(\Gamma)}$ are coefficient functions, $\Gamma$ matrices are possible structures for fourfermion operators and $G_{\mu \nu} \equiv g_{s} G_{\mu \nu}^{a} T^{a}$. All masses and couplings are functions of the normalization $\mu$, and so are the coefficient functions.

Therefore the Lagrangian is represented as an expansion in the inverse powers of the heavy-quark mass, with operators of dimension five and higher, which are due to hard gluon contributions, suppressed by the expansion parameter. However, further 
simplification is possible if the first (tree-level) term, $\mathcal{L}_{\text {heavy }}^{0}=\bar{Q}\left(i \gamma^{\mu} D_{\mu}-m_{Q}\right) Q$, is also studied carefully. The background gluon field $A_{\mu}$, which appears in the covariant derivative $D_{\mu}$, is weak compared to scale $m_{Q}$. This means that the heavy-quark field $Q(x)$ has a mechanical time-dependent factor associated with the rest energy $m_{Q}$, which can be factored out as:

$$
Q(x)=\mathrm{e}^{-i m_{Q} v_{\mu} x^{\mu}} \tilde{Q}(x), \quad v_{\mu}=p_{\mu} / M_{H_{Q}} .
$$

Here $v_{\mu}$ is the four-velocity and $M_{H_{Q}}$ is the mass of the hadron $H_{Q}$. Introducing the momentum operator $\pi_{\mu}$ for the rescaled field $\tilde{Q}$, through the relation:

$$
i D_{\mu} Q(x)=\mathrm{e}^{-i m_{Q} v_{\mu} x^{\mu}}\left(m_{Q} v_{\mu}+i D_{\mu}\right) \tilde{Q}(x) \equiv \mathrm{e}^{-i m_{Q} v_{\mu} x^{\mu}}\left(m_{Q} v_{\mu}+\pi_{\mu}\right) \tilde{Q}(x)
$$

the tree-level term $\mathcal{L}_{\text {heavy }}^{0}$ simply becomes [19]:

$$
\begin{aligned}
\mathcal{L}_{\text {heavy }}^{0} & =\sum_{Q} \varphi_{Q}^{+}\left\{\left(i D_{0}-m_{Q}\right)-\frac{1}{2 m_{Q}}\left(\vec{\pi}^{2}+\vec{\sigma} \vec{B}\right)\right. \\
& \left.-\frac{1}{8 m_{Q}^{2}}[-(\vec{D} \vec{E})+\vec{\sigma} \cdot\{\vec{E} \times \vec{\pi}-\vec{\pi} \times \vec{E}\}]+\mathcal{O}\left(1 / m_{Q}^{3}\right)\right\} \varphi_{Q},
\end{aligned}
$$

where $\vec{\sigma}$ denote the Pauli matrices. $E_{i}=G_{i 0}$ and $B_{i}=-(1 / 2) \epsilon_{i j k} G_{j k}$ are the background chromoelectric and chromomagnetic fields. The $\varphi_{Q}$ are now non-relativistic fields, connected to the rescaled fields $\tilde{Q}$ by the Foldy-Wouthuysen transformation [20]:

$$
\varphi_{Q}(x)=\left(1+\frac{\vec{\pi}^{2}+\vec{\sigma} \vec{B}}{8 m_{Q}^{2}}+\cdots\right) \frac{1+\gamma_{0}}{2} \tilde{Q}(x)
$$

The non-relativistic Lagrangian of Equation 1.8 is very similar to the non-relativistic expansions of quantum electrodynamics studied extensively in the last century [20]. As such, within its framework, many quantities associated with a given hadron (or a family of hadrons) can be computed relatively easily as functions of a few parameters (matrix elements of a few operators), values of which can be determined from experiment. 
As an example, it can be shown that the mass of a given heavy hadron can be expressed to order $1 / m_{Q}$ as [19]:

$$
M_{H_{Q}}=m_{Q}+\bar{\Lambda}+\frac{\left(\mu_{\pi}^{2}-\mu_{G}^{2}\right)_{H_{Q}}}{2 m_{Q}}+\cdots
$$

where $\mu_{\pi}^{2}$ and $\mu_{G}^{2}$ are the matrix elements for the dimension-5 operators in Equation 1.8. $\bar{\Lambda}$ is parameter associated with those terms in the effective Lagrangian that are entirely due to the light degrees of freedom; it can be considered as the effective mass of the light cloud around the heavy quark. It is interesting to note that $\mu_{\pi}^{2}=\left\langle H_{Q}\left|\vec{\pi}^{2}\right| H_{Q}\right\rangle$ is a measure of the kinetic energy of the heavy quark due to its Fermi motion inside the hadron, and $\mu_{G}^{2}=-\left\langle H_{Q}|\vec{\sigma} \vec{B}| H_{Q}\right\rangle$ relates to the amount of chromomagnetic field produced by the light cloud at the position of the heavy quark.

Noting that it is the chromomagnetic effects that separate the otherwise degenerate states $B$ and $B^{*}$, one can relate the masses of these two mesons using Equation 1.10, $M_{B^{*}}-M_{B} \simeq 2 \mu_{G}^{2} / 3 m_{b}$, where $\mu_{G}^{2} \equiv \mu_{G}^{2}(B)=-3 \mu_{G}^{2}\left(B^{*}\right)$. This relation can be used to determine the value of $\mu_{G}^{2}$ to order $1 / m_{b}$ from the measured masses of the two mesons and can be used in the computations for predicting other properties of the $B$-meson family.

\section{7 $\quad B \rightarrow X_{s} \gamma$ Photon Spectrum}

Due to experimental constraints to be discussed in the next section, it is not possible to measure the total branching fraction down to $E_{\gamma}>1.6 \mathrm{GeV}$, for which the theoretical predictions exist. Therefore extrapolations are necessary from the higher-energy part of the photon spectrum. Fortunately, the differential decay rate as a function of photon energy is fundamentally indifferent to the underlying physics processes, so this extrapolation can not directly harm the searches for physics beyond the Standard Model. On the other hand, the uncertainties on the calculation of the spectrum might significantly undermine the advantages of the high precision that have been achieved in the computation of the total decay rate.

Without the strong interactions, the photon from the $B \rightarrow X_{s} \gamma$ decay would 
be monochromatic with $E_{\gamma}^{b-\text { rest }}=m_{b} / 2$ in the rest frame of the $b$-quark. However, QCD strongly modifies this simple picture in two ways. First, real gluon emission, as discussed in Section 1.4, makes the decay multi-body. Second, due to its confinement inside the $B$ meson, the $b$ quark has a Fermi momentum, which leads to a dispersion of the photon spectrum.

The first effect can be computed by using the operator product expansion. However, towards the parton-model end-point of the spectrum, i.e.. for $E_{\gamma} \simeq m_{b} / 2$, the OPE cannot be used to predict the differential decay rate, because it fails to converge reasonably, being really an expansion in powers of $\Lambda_{Q C D} /\left(m_{b}-2 E_{\gamma}\right)$ and not $\Lambda_{Q C D} / m_{b}$.

The early predictions for the photon spectrum were made using a phenomenological spectator model [21] to implement the dispersion due to the Fermi motion. More recently, the predictions for these bound-state effects have been put on to firmer ground by using shape functions (or heavy-quark distribution functions) that are motivated by the resummation of an infinite number of leading-twist corrections meant to remedy the singularities encountered in the OPE [22]. Shape functions share many similarities with the parton distributions used in deep inelastic scattering.

Therefore in the past few years, the common methodology for computing the photon spectrum has been to calculate the parton-level spectrum using OPE and then convoluting this parton-level calculation with a shape function. The exact form of the shape function is not known yet, therefore one assumes some sort of well-behaved form for it, such as exponential or Gaussian, and require that its moments agree with various properties of the $B$ meson as computed in HQE. It should be mentioned that the shape function should be a universal, i.e. process-independent characteristic of the $B$ meson governing the inclusive decay spectra with massless partons in the final state, so it should be the same for $B \rightarrow X_{s} \gamma$ and $B \rightarrow X_{u} \ell \nu$.

One example of such a full spectrum computation, which has been used as a common ansatz in the experimental community, is by Kagan and Neubert [23]. This computation uses the next-to-leading-logarithmic precision calculations for the second, seventh and eighth components of the OPE for the parton-level spectrum. These 
are three of the largest contributors to the $b \rightarrow X_{s}^{\text {parton }} \gamma$ decay rate. (The contributions from the operators $\mathcal{O}_{3,4,5,6}$ are very small, because their Wilson coefficients $\left|C_{3,4,5,6}\left(\mu_{b}\right)\right|<0.07$, whereas $\left|C_{1,2,7,8}\left(\mu_{b}\right)\right| \simeq(0.5,1,0.3,0.15)$.) Then a shape function is assumed of some exponential form, which has a zero first moment and a second moment equal to $\frac{1}{3} \mu_{\pi}^{2}$, in accordance with HQE [24].

The resulting photon spectrum, as divided into its contributions from various OPE components, is shown in Figure 1.2. It should be emphasized that except for the very small $\left|C_{8}\left\langle\mathcal{O}_{8}\right\rangle\right|^{2}$ contribution, the different components have very similar spectral shapes, clearly indicating that the shape of the spectrum is not sensitive to underlying physics process, as mentioned earlier.

In the last two years, the Kagan and Neubert calculation has been superseded by two new calculations [25, 26], which are meant to address some flaws identified with its low-energy behaviour. Some details about these can be found in Appendix A The extrapolation of the experimental results is performed using fits to these more accurate calculations, while Kagan and Neubert calculation is still used in signal modelling.

\subsection{Signal Models}

A common feature for the theoretical computations of the photon spectra is that they predict a smooth energy distribution that monotonously increases from $1.6 \mathrm{GeV}$ up to around $2.5 \mathrm{GeV}$ and then monotonously falls off towards the kinematic endpoint at $E_{\gamma}=m_{B} / 2$, as seen in Figure 1.2. In reality, however, the $X_{s}$ system does not simply stand for a continuum of massless states, but for a mixture of $K^{*}$ resonances and various non-resonant states with net strangeness [27]. In particular, the lowest mass state $K^{*}(892)$, which will be referred to simply as $K^{*}$, results in a clear peak at the lower end of the $X_{s}$-invariant-mass spectrum.

While the sum of the contributions from the higher-mass states can be approximated by the continuous theoretical spectrum, the $B \rightarrow K^{*} \gamma$ mode needs a special 

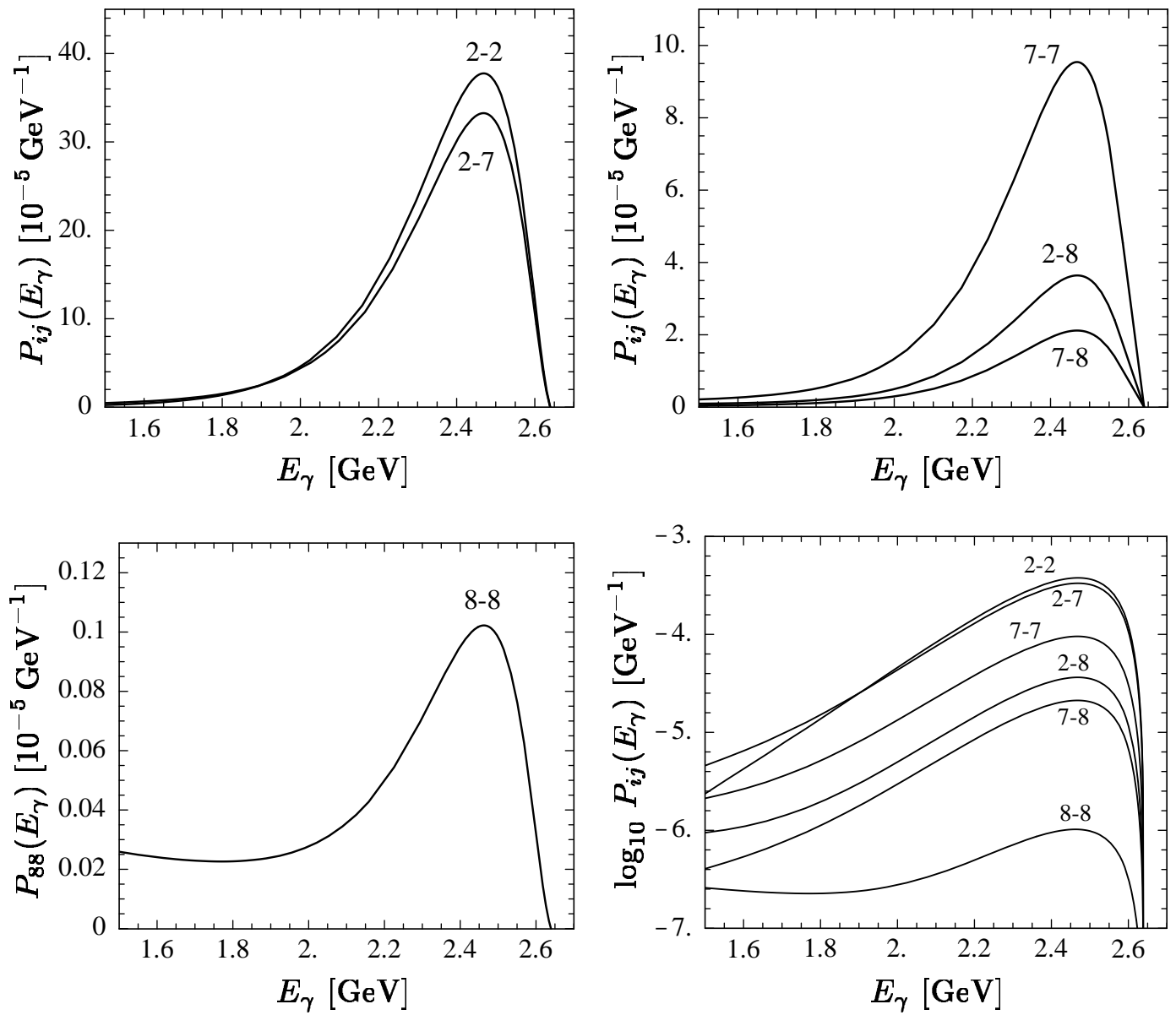

Figure 1.2: Contributions from various operators to $B \rightarrow X_{s} \gamma$ photon energy spectrum in the $B$-meson rest frame [23]. The label $i-j$ refers to the contribution to the decay amplitude associated with the combination $\mathcal{O}_{i} \mathcal{O}_{j}^{*}$, taking the corresponding Wilson coefficients into account. 
treatment in Monte Carlo simulations and in comparisons of theory with experiments. The present established method is known as the Kagan and Neubert prescription [23]. In this method, the theoretical $m_{X_{s}}$ spectrum below a certain mass cutoff, $m_{X_{s}}<m_{\text {cutoff }}$, is replaced by a relativistic Breit-Wigner distribution for the $K^{*}$. This explicit $K^{*}$ contribution is normalized to have the same integral as the part of the theoretical spectrum that is removed.

Following the results of an earlier BABAR measurement that reconstructs exclusive final states [28], the mass cutoff is chosen as $m_{\text {cutoff }}=1.1 \mathrm{GeV} / c^{2}$. For each theoretical photon spectrum considered, the $K^{*} \gamma$ fraction, $f_{K^{*}}$, is defined as the integral of the predicted photon energy distribution above $E_{\gamma}>2.525 \mathrm{GeV}$ divided by the total integral above $E_{\gamma}>1.6 \mathrm{GeV}$. The simple two-body kinematics of the $B \rightarrow X_{s} \gamma$ decay relate the invariant mass of the $X_{s}$ system to the energy of the photon through the relation $m_{X_{s}}=\sqrt{m_{B}^{2}-2 m_{B} E_{\gamma}}$.

The branching fractions for the exclusive decays $B^{0} \rightarrow K^{* 0} \gamma$ and $B^{ \pm} \rightarrow K^{* \pm} \gamma$ have been measured for $m_{X_{s}}<1.1 \mathrm{GeV}$ in the data sample used for the inclusive analysis [29]:

$$
\begin{gathered}
\mathcal{B}\left(B^{ \pm} \rightarrow K^{* \pm}(892) \gamma\right)=(3.87 \pm 0.28(\text { stat }) \pm 0.26(\text { syst })) \times 10^{-5} \\
\mathcal{B}\left(B^{0} \rightarrow K^{* 0}(892) \gamma\right)=(3.92 \pm 0.20(\text { stat }) \pm 0.24(\text { syst })) \times 10^{-5}
\end{gathered}
$$

The average of these two values (the inclusive measurement does not make a distinction between charged and neutral $B$ mesons) and the Kagan and Neubert prescription gives a natural normalization for any arbitrary theoretical spectrum. For instance for the Kagan and Neubert calculation with input parameters $m_{b}=4.8 \mathrm{GeV} / c^{2}$ and $\mu_{\pi}^{2}=0.30 \mathrm{GeV}^{2}$, the $K^{*} \gamma$ fraction is $f_{K^{*}}=15.17 \%$ and thus the assumed total branching fraction is $2.6 \times 10^{-4}$. It must be clear that this normalization, apart from being used as a basic guideline in Monte Carlo simulation studies, is completely arbitrary and its use has no effects on the values extracted in the experiment.

Any given theoretical calculation with the $K^{*} \gamma$ component added in accordance to the Kagan and Neubert prescription and the above described normalization will be referred to as a signal model. For instance the "KN480 model" is the model described in the previous paragraph. It is taken as the default signal model in this experiment. 


\begin{tabular}{|c|c|c|c|}
\hline Model & $m_{b}\left(\mathrm{GeV} / c^{2}\right)$ & $\mu_{\pi}^{2}\left(\mathrm{GeV}^{2}\right)$ & $f_{K^{*}}$ \\
\hline KN455 & 4.55 & 0.70 & 0.0731 \\
KN460 & 4.60 & 0.60 & 0.0832 \\
KN465 & 4.65 & 0.52 & 0.0964 \\
KN480 & 4.80 & 0.30 & 0.1517 \\
\hline
\end{tabular}

Table 1.1: The input parameters $\left(m_{b}\right.$ and $\left.\mu_{\pi}^{2}\right)$, and the assumed fraction of $K^{*}(892) \gamma, f_{K^{*}}$, relative to the total $B \rightarrow X_{s} \gamma$ branching fraction for $E_{\gamma}>1.6 \mathrm{GeV}$, for various parameterizations of the Kagan and Neubert $(\mathrm{KN})$ signal model.

Many other models are used for systematic studies. Table 1.1 lists some of these, others are given in Appendix @. To simplify the discussions, each model has been assigned a mnemonic that starts with the first letters of the names of the theorists whose calculations have been used (BBU for Benson, Bigi, Uraltsev and LNP for Lange, Neubert, Paz).

\subsection{Experimental Techniques}

It is clear that both the calculation of the parton level $b \rightarrow s \gamma$ and its connection to the meson level $B \rightarrow X_{s} \gamma$ present great theoretical challenges. These challenges are met by equally difficult ones on the experimental side. Although the hard photon in the final state is a distinctive signature of the decay, there are many other processes with equally energetic photon final states, which overwhelm the $B \rightarrow X_{s} \gamma$ signal.

The first observation of a $b$-penguin decay was the exclusive $B \rightarrow K^{*} \gamma$ in 1993 [30]. Inclusive $B \rightarrow X_{s} \gamma$ measurements have so far been possible only at the $Z$-pole [31] or at the $\Upsilon(4 S)$ resonance. Despite the large difference in the c.m. energies, the principal background processes and the techniques used to reduce them are very similar.

Since $B \rightarrow X_{s} \gamma$ is a rare decay (with a branching fraction less than $0.1 \%$ ), it is not surprising to see that the signal photons are overwhelmed by background from a multitude of background processes (Figure 1.3). These backgrounds can be organized into two major categories. 


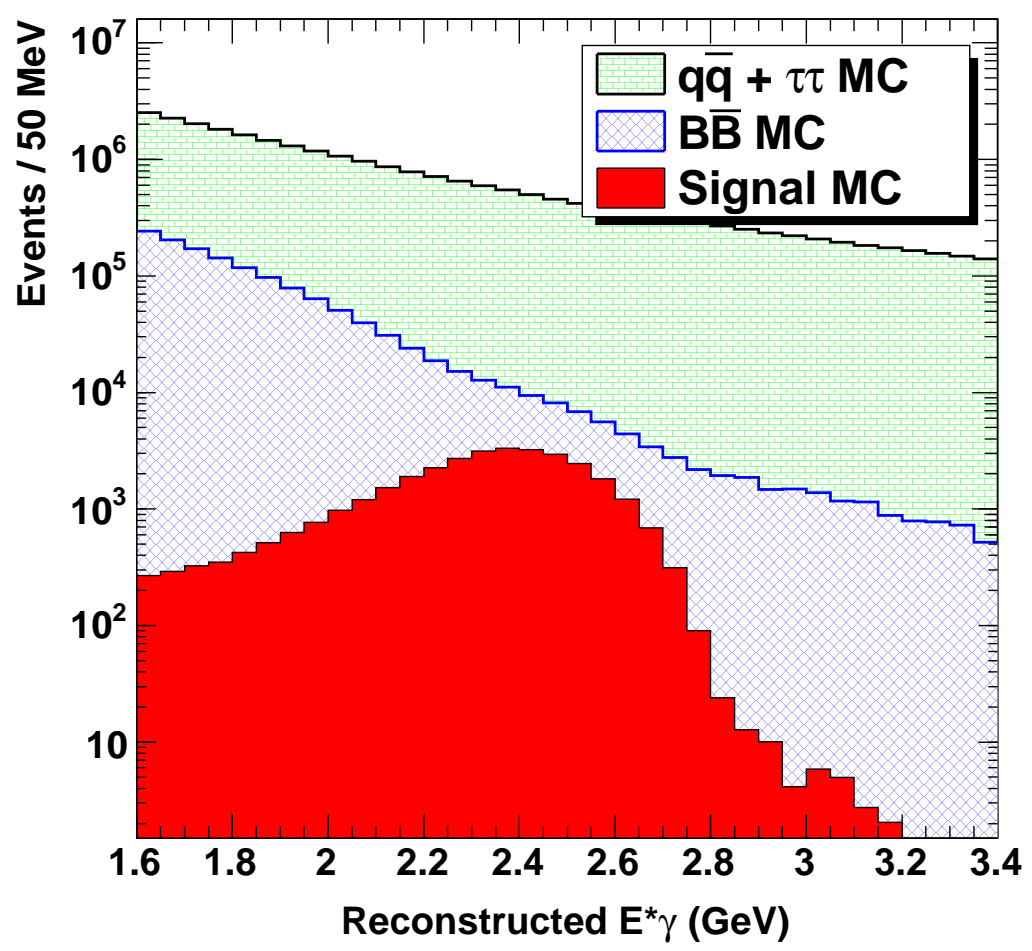

Figure 1.3: Simulated photon energy in the $e^{+} e^{-}$center-of-mass frame at $\Upsilon(4 S)$ resonance. Plotted are photon candidates from signal (red), other $B \bar{B}$ (blue), $q \bar{q}$ and $\tau^{+} \tau^{-}$(green) Monte Carlo events. Additional backgrounds from (radiative) Bhabha scattering, radiative $\mu^{+} \mu^{-}$and other QED processes are not shown. 
The $b \bar{b}$ backgrounds (which will be referred to as $B \bar{B}$ backgrounds, since $B$-mesons are the only $b$-containing hadrons for experiments such as BABAR running at the $\Upsilon(4 S)$ resonance) consist of photons from various other $b$-quark decays. Most of these backgrounds are from energetic $\pi^{0}$ or $\eta$ mesons that originate in prompt decays or from secondary decays such as $B \rightarrow D^{*} \rho^{ \pm}, \rho^{ \pm} \rightarrow \pi^{0} \pi^{ \pm}$. These backgrounds are usually difficult to separate from the signal events since their signatures in the detectors closely resemble the signal events. (A signal event is a $B \bar{B}$ event in which at least one of the $B$ mesons decays to $X_{s} \gamma$.)

All the remaining backgrounds (except for the almost negligible beam-generated and cosmic ray backgrounds) are from lighter processes. Both at the $Z$-pole and at the $\Upsilon(4 S)$ resonance where the measurements have been possible, the $b$-quark is the heaviest fundamental fermion that can be produced, so any collision that does not result in a $b \bar{b}$ pair, has to progress with lighter particles appearing at the parton level. Even when a $\tau^{+} \tau^{-}$pair is created (with $\tau$ lepton being the heaviest fundamental fermion after the $b$-quark), the resulting event tends to be more jet-like due to the extra kinetic energy available to each of the $\tau$ leptons. The resulting differences in the event topology are particularly distinguishing for experiments running at the $\Upsilon(4 S)$ resonance, where the $B$-mesons are almost at rest in the center-of-mass frame of the collision (Figure 1.4).
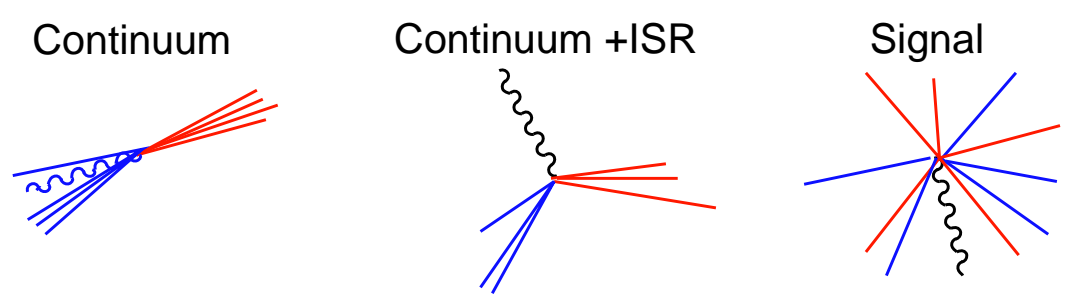

Figure 1.4: The event topology for background and signal events [32]. Continuum background events are shown without (left) and with (middle) initial state radiation. The $B \bar{B}$ events (including the signal events) have an isotropic topology (right).

Similar to $B \bar{B}$ backgrounds, the main sources of background photons in $q \bar{q}$ continuum processes are the decays of the $\pi^{0}$ and $\eta$ mesons. However there is also an 
important contribution from initial state radiation, where a high energetic photon is created before the creation of the $q \bar{q}$ pair. It is more difficult to separate these ISR events from the $B \rightarrow X_{s} \gamma$ signal, since the energy available to the $q \bar{q}$ jets after the radiation is lower. For $\tau^{+} \tau^{-}$events, the event topology after ISR can look particularly isotropic, but fortunately, these events are not too common. Furthermore, they tend to have low multiplicity (the total number of particles in an event) and can thus be further suppressed. The same can also be said for the QED-continuum backgrounds, which mostly consist of radiative $e^{+} e^{-}$(Bhabha) and $\mu^{+} \mu^{-}$events.

A common technique to all experiments for reducing the backgrounds significantly is known as the $\pi^{0}(\eta)$ veto. In this technique every signal-candidate photon is paired with any other photon in the event and is vetoed if the combination has an invariant mass close to the nominal mass of a $\pi^{0}$ or $\eta$ meson. Since the $\pi^{0}$ and $\eta$ decays constitute a large part of both $B \bar{B}$ and continuum backgrounds, a factor of two or better improvement in the overall signal-to-background ratio is not uncommon with the veto technique.

While the improvements from the event-topology and particle-multiplicity requirements, and the light-meson vetoes are significant, they are far from reducing the total amount of backgrounds to the level of the signal. Depending on the additional strategy applied, various experiments can be organized into two broad groups:

Sum-of-exclusive modes. In this method the $B \rightarrow X_{s} \gamma$ decay tree is fully reconstructed from the detected particles. Each photon with high enough energy is combined with a list of other particles with net strangeness and the invariant mass of the combination is required to be agree with the nominal mass of a $B$-meson (or any other $b$-containing hadron for the $Z$-pole experiments). These kinematic requirements significantly reduce the backgrounds. The residual backgrounds can be estimated using fits to the kinematic distributions.

Fully-inclusive measurements. No requirements are imposed on the $X_{s}$ system. The backgrounds are measured in independent data samples or estimated using Monte Carlo simulation and then subtracted.

While the first strategy seems to be particularly straight-forward and less prone to systematic biases, the precision that can be achieved with it has a major limiting 
factor. While many of the different decay modes can be added, there are missing modes that are very difficult to reconstruct. Unfortunately, there are no accurate theoretical models that predict the fraction of all the different $X_{s}$ states that have not been detected. Estimates have to be made using the data itself, which introduces significant systematic errors.

The second strategy is the one chosen here. Unfortunately the technique is not applicable by itself directly, since it requires additional samples of signal-free data for background subtraction and this may not be available, or as in the case of BABAR experiment, might be limited in amount. Therefore in order to achieve good postsubtraction precision, the fully-inclusive strategy is commonly coupled with some other means of reducing the backgrounds. In this analysis, this will be done using a lepton tagging method. 


\section{Chapter 2}

\section{The BABAR Experiment}

The BABAR detector is a multi-layer particle detector designed mainly to study timedependent $C P$-violating asymmetries in the decay of neutral $B$ mesons to $C P$ eigenstates. Built and operated by an international collaboration of more than 550 physicists and engineers, it has recorded over 250 million $e^{+} e^{-} \rightarrow \Upsilon(4 S)$ events since 1999.

To achieve high precision in the time-dependent $C P$ analyses, the design requires large solid angle coverage, micron-precision vertex location, good kaon-pion separation at multi-GeV momenta and high accuracy electromagnetic calorimetry. The versatility afforded by such features and the considerable size of the data sample allows a wealth of other scientific avenues to be explored. Precision measurements of bottom and charm mesons and of $\tau$ leptons, and searches for exotic states and rare processes are possible.

In this chapter, the PEP-II collider and the main features of the BABAR detector will be introduced. Further details can be found elsewhere [33].

\subsection{The PEP-II $B$ Factory}

The PEP-II $B$ Factory, is an asymmetric-energy electron-positron collider, located in the Positron-Electron Project (PEP) tunnel at the Stanford Linear Accelerator Center, Stanford, CA. Constructed by a collaboration from SLAC, LBNL and LLNL, it has been providing data to the BABAR experiment since 1999 . 


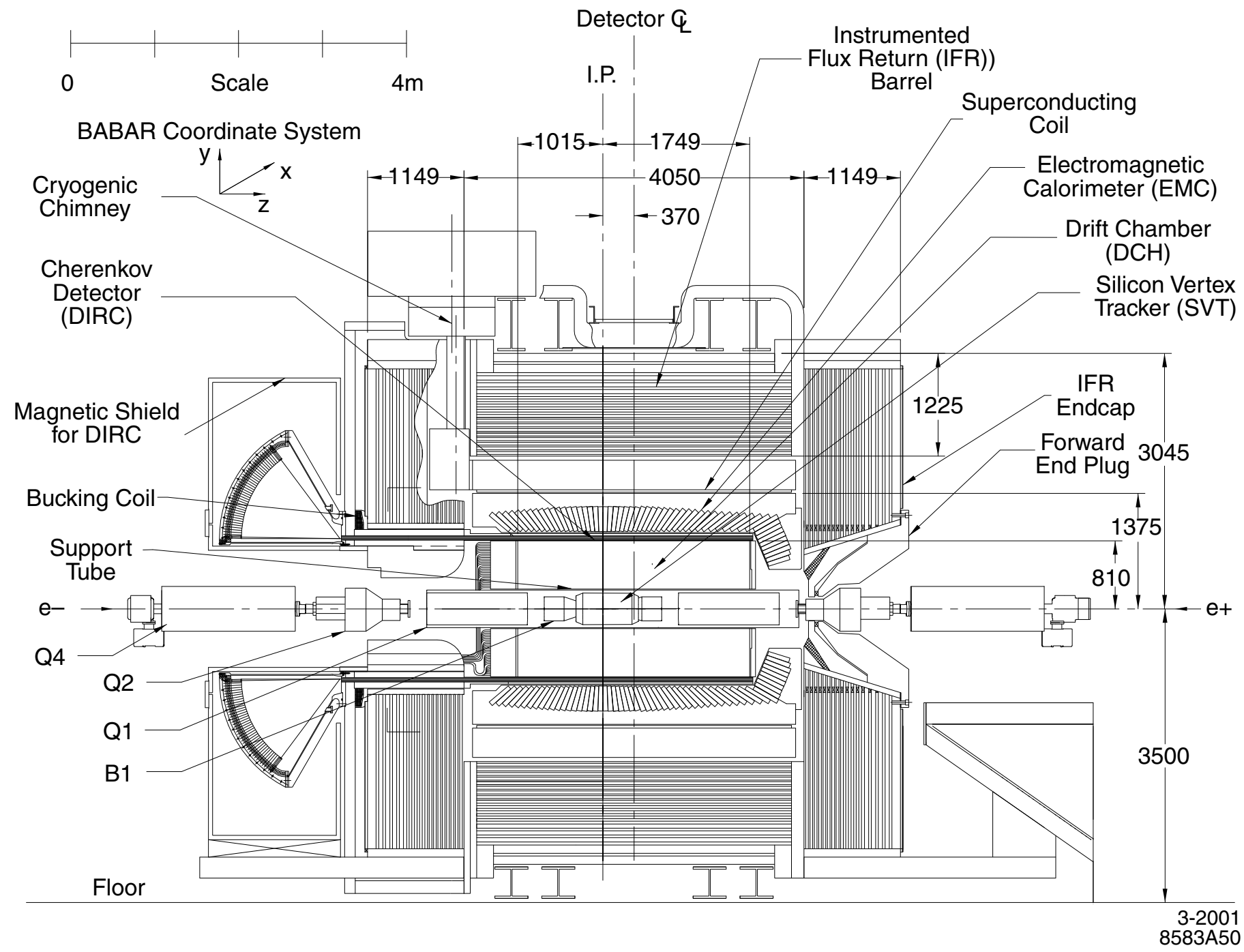

Figure 2.1: Longitudinal section and the coordinate system of the BABAR detector [33. Dimensions are given in millimeters. 
The collider consists of two 2.2-km-circumference storage rings, into which electrons and positrons from the SLAC linac are injected. The high-energy ring (HER) stores the $\sim 1.5 \mathrm{~A}$ electron beam at $9.0 \mathrm{GeV}$. The low-energy ring (LER) operates at a record high circulating current of $\sim 2.5 \mathrm{~A}$, and houses the $3.1 \mathrm{GeV}$ positrons. The asymmetry in beam energies leads to a Lorentz boost to the center-of-mass (c.m.) frame of $\beta \gamma=0.56$, which makes it possible to reconstruct the decay vertices of the two $B$ mesons that are produced. Since inclusive measurements does not require the reconstruction the $B$-meson vertices, this feature is not directly relevant for them.

The c.m. energy of $10.58 \mathrm{GeV}$ corresponds to the mass of the $\Upsilon(4 S)$ resonance. Since this resonance decays exclusively into $B^{+} B^{-}$and $B^{0} \bar{B}^{0}$ pairs 34, the crosssection for $B \bar{B}$ production is approximately $1.1 \mathrm{nb}$. Therefore, at the design luminosity of $3 \times 10^{33} \mathrm{~cm}^{-2} \mathrm{~s}^{-1}$, PEP-II can deliver on the order of $10^{8} \mathrm{~B}$ mesons per year. To achieve and surpass this goal, the main impediments have been the electron cloud around the positron bunches and the self-blowup of the positron beam during high-current conditions. These problems have been found to be inter-related and put under control by tailoring the bunch patterns, synchrotron-radiation scrubbing of the vacuum surfaces and winding axial-field solenoids around the accessible sections of the LER vacuum chamber. With these and other improvements, instantaneous luminosities of up to $9.2 \times 10^{33} \mathrm{~cm}^{-2} \mathrm{~s}^{-1}$ have been reached [36].

Throughout the data taking, the beam energies (which have a few MeV spread), the direction of the beams relative to detector central axis (which is $20 \mathrm{mrad}$ off), the location of the beam-beam interaction point (IP, which is approximately $37 \mathrm{~cm}$ offset from the detector center) and the amount of beam backgrounds are carefully tracked and recorded. In addition, about one-tenth of the data, referred to as off-resonance data, are taken at a c.m. energy $40 \mathrm{MeV}$ below the $\Upsilon(4 S)$ resonance (hence lower than the threshold for $B \bar{B}$ production) to allow for studies of non-resonant background.

\subsection{The BABAR Tracking System}

The BABAR tracking system consists of a silicon strip detector and a central drift chamber located inside a $1.5 \mathrm{~T}$ magnetic field. 


\subsubsection{The BABAR Magnet System}

A strong and uniform magnetic field is essential for the identification and momentummeasurements of charged particles. For the BABAR experiment, a $1.5 \mathrm{~T}$ field is maintained by a magnet system that consists of:

- a liquid-helium-cooled super-conducting solenoid made up of niobium-titanium Rutherford cable co-extruded with aluminum stabilizer, with an operating current of about $4.6 \mathrm{kA}$,

- a segmented flux return of $\sim 55 \mathrm{~cm}$-thick steel, which also serves as the hadron absorber for hadron/muon separation (section 2.5), and,

- a water-cooled copper bucking coil, which provides approximately $98 \%$ compensation for the stray fields that might otherwise disturb the operation of DIRC photomultipliers and the fields of the PEP-II dipole (B1) and quadrupole (Q1, Q2, Q4, Q5) magnets (Figure 2.1).

The magnetic field has been mapped out with Hall and NMR magnetometers and parameterized with a polynomial of degree up to 40 in $r$ and $z$. It is very uniform in the tracking volume, with the azimuthal component never exceeding $1 \mathrm{mT}$. For a high-momentum particle, the variation of the field transverse to the trajectory is always less than $2.5 \%$. (The right-handed coordinate system, with the $z$-axis defined as the principle axis of the drift chamber (DCH), is shown in Figure 2.1])

\subsubsection{Silicon Vertex Tracker}

The Silicon Vertex Tracker (SVT) is the innermost component of the BABAR detector, which allows precise reconstruction of charged particle trajectories and decay vertices near the interaction region. It has been designed to satisfy the geometric and mechanical constraints of the PEP-II B1 magnets and the support tube (all components inside the tube should have long mean-time-to-failure, since SVT is inaccessible during normal operations), the resolution requirements for time-dependent $C P$ asymmetry measurements (spatial z-resolution on the order of $100 \mu \mathrm{m}$ ) and the demand 
for providing standalone tracking for particles with low transverse momentum (the drift chamber alone cannot reliably measure $p_{T}$ below $120 \mathrm{MeV}$ ). Finally, these design goals have to be achieved in a high-radiation environment.

The SVT consists of 52 modules of double-sided silicon sensors organized into 5 concentric layers around the beam axis. The three innermost layers (at radii of 32, 40 and $54 \mathrm{~mm}$ ) run parallel to the beam pipe, whereas the modules in the two outer layers are arranged in an arch to increase the crossing angle for particles near the edge of acceptance (Figure 2.2). Each module is made up of four to eight $300 \mu \mathrm{m}$-thick high-resistivity $(6-15 \mathrm{k} \Omega \mathrm{cm})$ n-type silicon wafers, with $\mathrm{n}^{+}$and $\mathrm{p}^{+}$strips running orthogonally on opposite sides. Strips that run parallel to the beam are used for $\phi$ measurements, whereas the $z$-measuring strips are oriented transversely. The readout pitch varies between $50-210 \mu \mathrm{m}$, with the $\phi$-strips twice more densely readout than the $z$-strips. The total active silicon area is $0.96 \mathrm{~m}^{2}$, covering $90 \%$ of the solid angle in the c.m. frame.

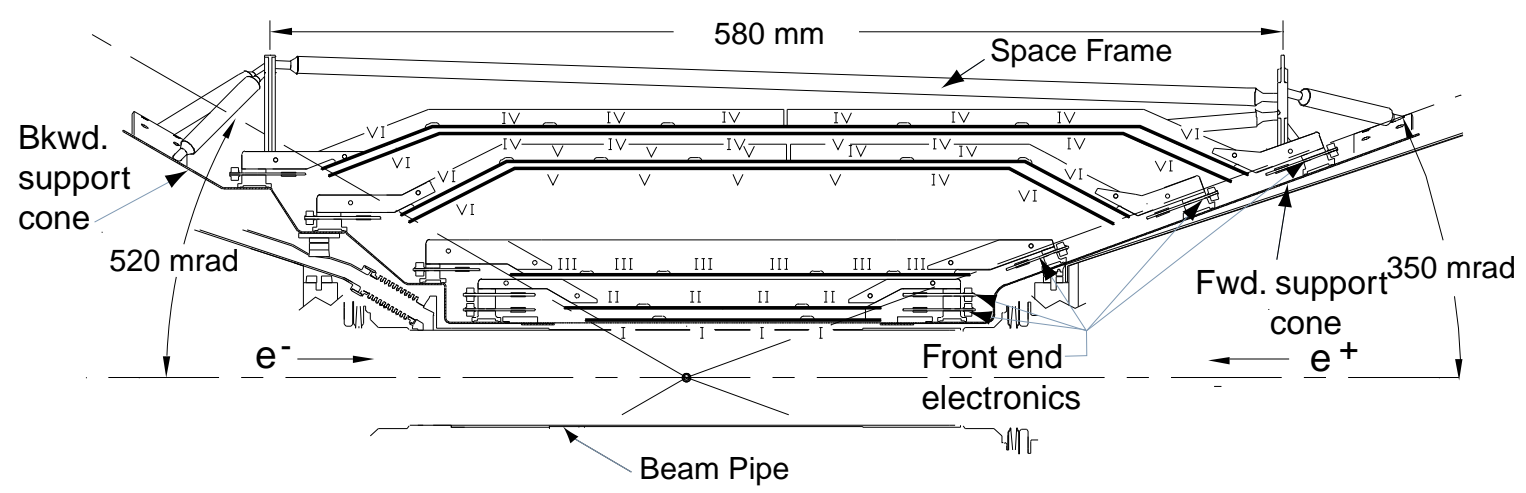

Figure 2.2: Longitudinal section of the silicon vertex detector [33. The roman numerals label the types of sensor sections with different geometrical shapes or surface areas. Type VI sections are trapezoidal in shape, whereas the others are rectangular.

For the readout, the signal from the strips are routed by flexible fanout circuits to custom-design CMOS ICs (AToM chip), which are capable of time-over-threshold measurement with a signal-to-noise ratio greater than 15 even for minimum ionizing particles (MIPs). Even though only about half of the strips are read-out (every 
other strip is frequently left floating in order to improve resolution at high Lorentz and incidence angles) and some strips are electrically ganged, the total number of channels is close to 150,000 . With so many channels, it is essential to locate the readout electronics entirely outside the detector volume. The mechanical support is provided by a carbon-fiber skeleton.

For the stable operation of the SVT, various parameters are carefully monitored and recorded. Temperature and humidity are maintained by external cooling of the beam pipe with chilled water and a flow of dry air through the support tube. The calibration of the electronics is performed daily, using the test charge injection circuitry of the AToM chips. The relative position of the SVT sensors with respect to each other is quite stable and has been determined from an optical survey during the assembly of the SVT. However, as needed on special occasions, for instance after magnet quenches and detector access, the local alignment is reassessed by fitting tracks from $e^{+} e^{-} \rightarrow \mu^{+} \mu^{-}$events and cosmic rays. Finally, the overall location of the SVT with respect to the global coordinate system defined by the DCH changes due to diurnal temperature variations etc. To account for this, the SVT is surveyed every $2-3$ hours and new calibration constants are determined by minimizing the difference between track parameters obtained with SVT-only and DCH-only fits.

The SVT has performed well since its commissioning. The combined hardware and software hit reconstruction efficiency of $97 \%$ has not deteriorated during the past years. Initially, 9 out of 208 readout sections were found to be defective, but during a 2002 shutdown, 5 of these defective sections were identified to be due to poor connections and fixed. Certain components have already accumulated more than $2 \mathrm{MRad}$ of radiation and there are increases in inter-strip capacitance and leakage currents along with shifts in the depletion voltage. However, tests with ${ }^{60} \mathrm{Co}$ source and $1 \mathrm{GeV}$ electron beam indicate that the SVT is likely to perform satisfactorily up to $5 \mathrm{MRad}$, even after Si type inversion [37.

The SVT has successfully achieved its goals. Along both $\phi$ and $z$ directions, the spatial hit resolution for all the modules varies between 10 to $40 \mu \mathrm{m}$, depending on the track incidence angle. For MIP tracks with at least four SVT hits, the mean $\mathrm{d} E / \mathrm{d} x$ resolution is approximately $14 \%$. 


\subsubsection{Drift Chamber}

As the other component of the BABAR tracking system, the drift chamber (DCH) complements the SVT by providing precise measurements of the charged particle momenta and by tracking the daughters of long-lived particles, like $K_{S}^{0}$, whose decay vertices fall outside the SVT volume. Moreover, DCH ionization loss $(\mathrm{d} E / \mathrm{d} x)$ measurements play an important role in particle identification, particularly for particles with low momenta and for particles that fall outside the DIRC acceptance.

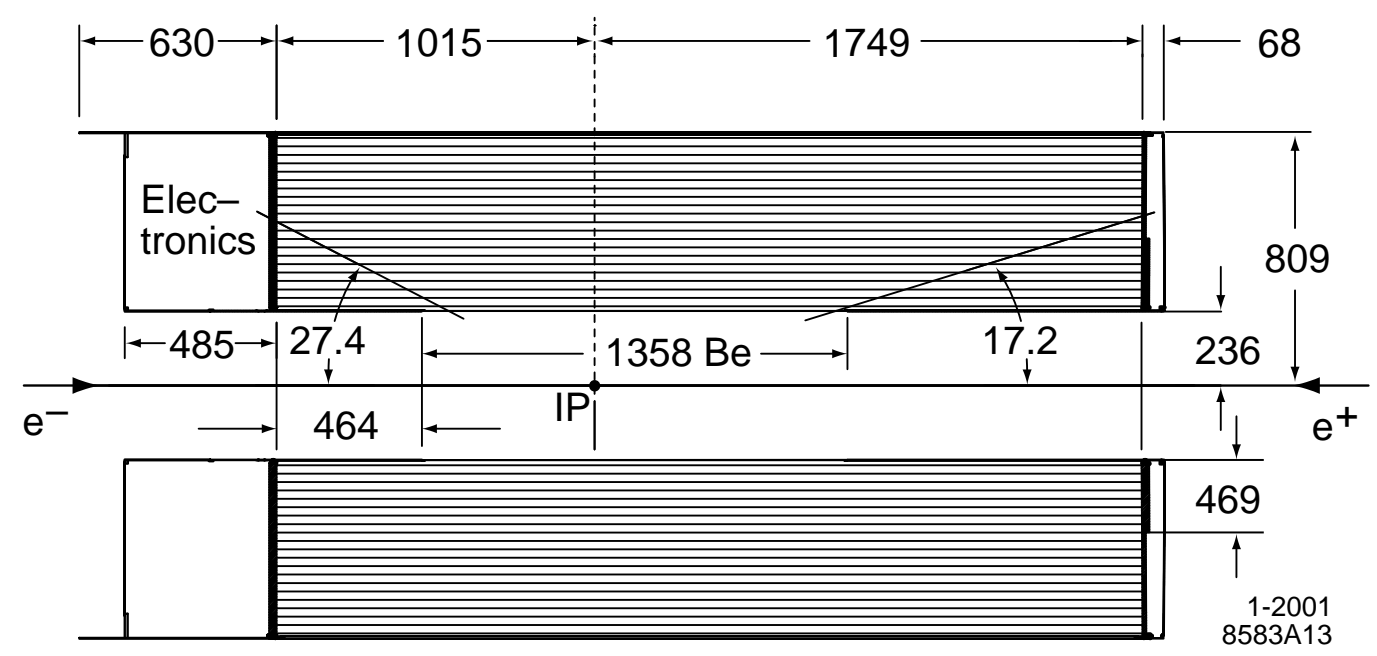

Figure 2.3: Longitudinal section of the drift chamber 33. Note that the chamber center is offset by $370 \mathrm{~mm}$ from the interaction point (IP). Dimensions are given in millimeters.

The DCH has a cylindrical structure, mostly constructed from aluminum, except for the $9 \mathrm{~mm}$-thick carbon-fiber-composite outer wall and the mid-section of the inner wall, which is made of $1 \mathrm{~mm}$-thick beryllium. It is almost $3 \mathrm{~m}$ long and positioned asymmetrically with respect to the IP (Figure 2.3), designed such that a high-momentum track on the edge of the SVT polar angle acceptance will pass through at least half of its 40 layers.

The layers of the DCH consist of 1.7 to $2 \mathrm{~cm}$-wide hexagonal drift cells. Each one of the 7104 cells has a gold-plated tungsten-rhenium sense wire at the center surrounded 


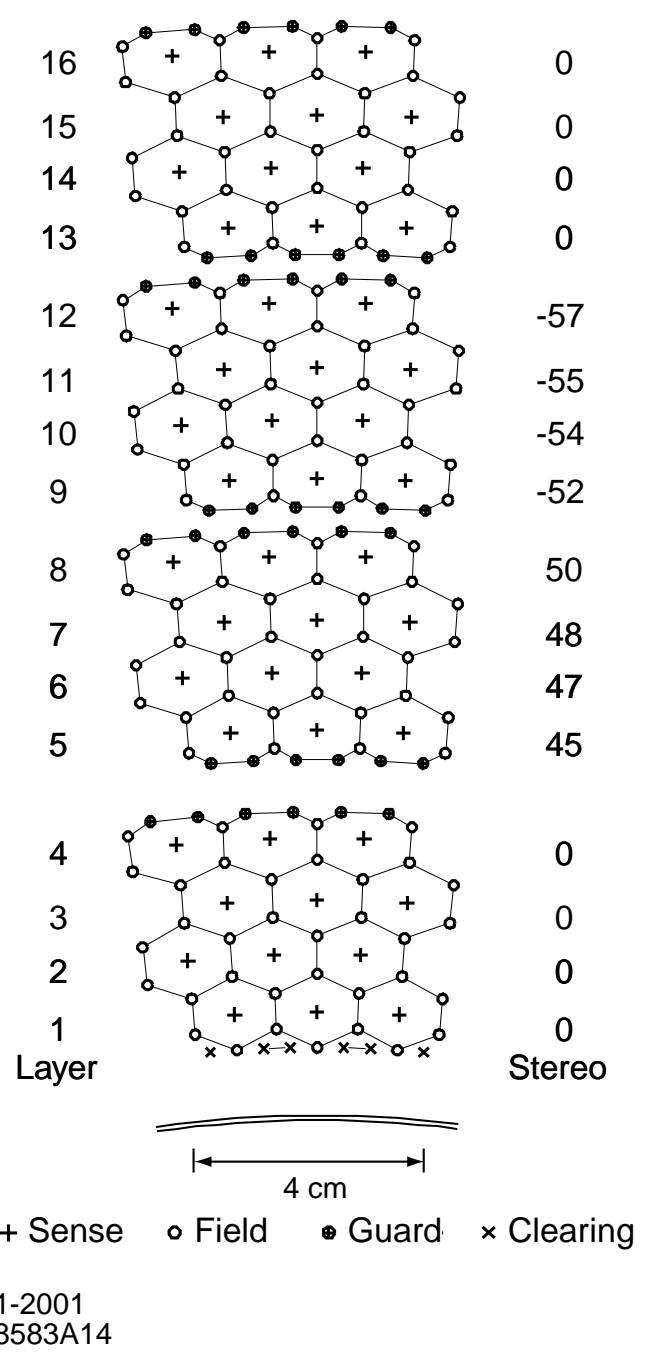

Figure 2.4: Schematic layout of the four innermost superlayers of the DCH [33]. The lines between field wires have been added in order to improve the visualization of drift cells. Stereo angles of each layer (the angle between the direction of the sense wire and the beam axis) are tabulated (in mrad) on the right side. Also shown are the beryllium inner wall and the clearing wires, which are used to collect charges from photon conversion in the walls. by six gold-plated aluminum field wires. The field wires are grounded, whereas the sense wires are operated at a high voltage (1900, 1930 or $1960 \mathrm{~V}$ in different runs periods). The layers are organized into 10 superlayers, with same wire orientation and equal number of cells in each layer of a superlayer. The stereo angles of the superlayers are alternated between axial (A) and stereo pairs (U,V), in the order AUVAUVAUVA, in order to obtain longitudinal position information (Figure 2.4).

The chamber is filled with a 80:20 helium: isobutane gas mixture, slightly above the atmospheric pressure. This choice of light gas mixture and the choice of aluminum for the field wires keep multiple scattering inside the $\mathrm{DCH}$ at a minimum. A small amount of water vapor $(3500 \mathrm{ppm})$ is added to prevent electrical discharges and damage to the wires.

The electronics for the chamber, both the service boards providing the electrostatic potentials for the wires and the front-end readout electronics, are located on the rear endplate. The DCH FEE consist of custom amplifier/digitizer boards, which perform 4-bit time and 6-bit charge measurements, and readout interface boards, which control the flow of data and trigger information into specific I/O modules.

For the reconstruction of the tracks, a crucial step is the translation of measured drift 
time into drift distance. The precise determination of the time-to-distance relation is performed with tracks from $e^{+} e^{-}$and $\mu^{+} \mu^{-}$events, where the drift distance for a given DCH signal is estimated by computing the track's distance of closest approach to the wire producing the signal. To avoid bias, the hit on the wire in consideration is not used in the reconstruction of the track. After corrections for the angle-dependent effects, the measured position resolution is as low as $100 \mu \mathrm{m}$ for drift distances of 3-7 mm. (It gets worse rapidly in the closer vicinity of the sense or the field wires).

The specific energy loss, $\mathrm{d} E / \mathrm{d} x$, for a charged particle transversing the $\mathrm{DCH}$ is computed as a truncated mean of the lowest $80 \%$ of the integrated charge deposit measurements from individual cells. Various corrections are applied on these measurements to account for changes in gas gain, differences in cell geometry, space charge build-up etc. After these corrections, studies of tracks from Bhabha scattering events yield an RMS resolution of $7.5 \%$.

\subsubsection{Overall Performance of the Tracking System}

Offline reconstruction starts by helix fits to DCH hits identified by the L3 tracking algorithm (section 2.6.2) for tracks originating from the IP. Then various sophisticated algorithms, which also identify tracks not originating from around the IP, are applied consecutively, at each step improving the measurement of the event start time $t_{0}$ and progressively cleaning the tracking environment. SVT track segments are added when they are found consistent with extrapolations from the DCH tracks. Kalman filter methods are used to improve fits. Remaining SVT hits are analyzed by complementary algorithms to identify SVT-only tracks.

Each reconstructed track is defined by five parameters measured at the point of closest approach (POCA) to the $z$-axis: $d_{0}$ and $z_{0}$ are the radial (in the $x-y$ plane) and longitudinal (along the $z$-axis) coordinates of this POCA, $\phi_{0}$ the azimuthal angle, $\lambda$ the dip angle relative to transverse plane, and $\omega=1 / p_{T}$ is the curvature. $d_{0}$ and $\omega$ can be set negative depending on the charge of the particle and its direction.

For transverse momenta above $200 \mathrm{MeV} / c$, the mean track reconstruction efficiency in the DCH is around $98 \%$, and exceeds $85 \%$ for the SVT. The FWHM resolutions 
for the track parameters, as determined from cosmic rays passing near the IP, are

$$
\begin{gathered}
\sigma_{d_{0}}=23 \mu \mathrm{m} \quad \sigma_{z_{0}}=29 \mu \mathrm{m} \quad \sigma_{\phi_{0}}=0.43 \mathrm{mrad} \quad \sigma_{\tan \lambda}=0.53 \times 10^{-3} \\
\sigma_{p_{T}} / p_{T}=(0.13 \pm 0.01) \% \cdot p_{T}(\mathrm{GeV} / c)+(0.45 \pm 0.03) \%
\end{gathered}
$$

where the position and angle resolutions are averages for tracks with momenta above $p_{T}$ of $3 \mathrm{GeV} / c$. The linear dependence of the $p_{T}$ resolution also holds at lower momenta.

\subsection{Ring-Imaging Cherenkov Detector}

The Detector of Internally Reflected Cherenkov Light (DIRC) is a third generation ring-imaging Cherenkov detector, designed to provide $3 \sigma$ or greater separation even for high-momentum pions and kaons from rare two-body decays of the $B$ mesons, like $B^{0} \rightarrow K^{ \pm} \pi^{\mp}$. It is meant to satisfy this design goal in a high background environment and without introducing too much material in front of the EMC, in order to minimize the degradation of the performance and the cost of the calorimeter.

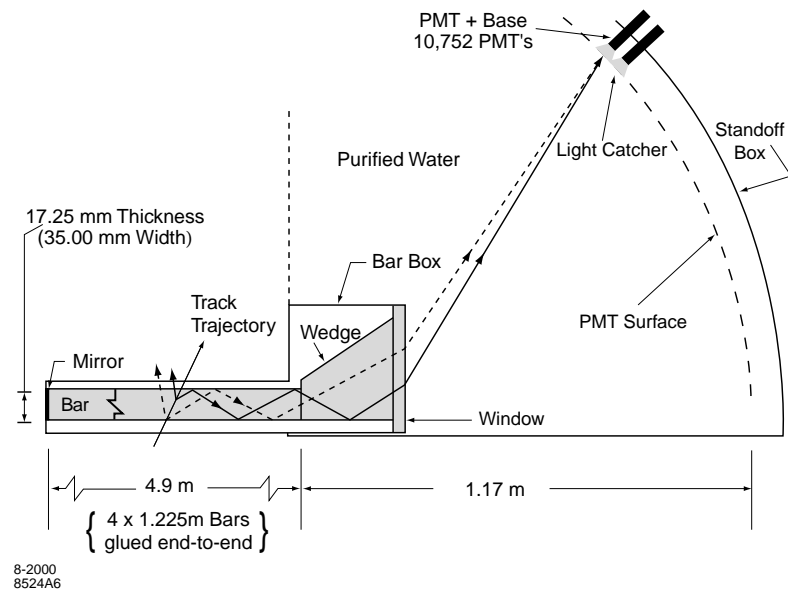

Figure 2.5: Diagram of the DIRC radiator bar and imaging region 33, illustrating the working principle of the detector.

The DIRC is based on the principle that fused silica that acts as the Cherenkov radiator can also serve as a light guide (Figure 2.5). Silica has high resistance to ionizing radiation, long attenuation length and low chromatic dispersion, and most importantly a high mean index of refraction $(n=1.473)$. When particles with $\beta \approx 1$ pass through a thin bar of fused silica, most Cherenkov photons lie within the total internal reflection limit and are transported to the ends of 
the bar. By placing a mirror on one end, and instrumenting the other with a large array of photomultiplier tubes (PMTs), it is possible to determine both the Cherenkov angle, $\theta_{c}$, and the azimuthal angle of a photon around the track direction.

The detector has a total of 144 such silica bars mounted in 12 bar boxes. All bar boxes open into a water-filled conic expansion region called a standoff box, which is instrumented by 12 sectors of 896 PMTs each. All PMTs are surrounded by light catcher cones, raising the total light collection fraction to about $90 \%$. The operating high voltage is varied for groups of PMTs to account for differences in their gains. The standoff box is located outside the flux return of the magnet system and enclosed by a steel shield (Figure 2.1). The field in the PMT region is below $1 \mathrm{G}$.

Water, with a chromaticity index matching that of silica and a reasonably close index of refraction $(n \approx 1.346)$ proves to be an inexpensive material to fill the standoff box. It is purified with filters and a reverse osmosis unit, de-gassed, de-ionized and exposed to UV radiation to prevent bacteria growth.

The DIRC front-end electronics (FEE), designed to measure the arrival time of each detected photon to an accuracy limited by the intrinsic PMT transit time spread (1.5 ns), consists of 168 boards processing 64 inputs each. While only one out of 64 channels is selected by a multiplexer to be digitized by an 8-bit flash ADC (ADC information is not needed for event reconstruction), custom made TDC ICs with $0.5 \mathrm{~ns}$ binning process all the channels. Input buffering and selective readout allow the selection of hits within \pm 300 ns of trigger signals.

Two types of calibration, a daily light pulser calibration and an online data stream calibration based on expected and measured arrival times for reconstructed tracks from collision data, are essential for the operation. Voltage supplies for the PMTs and the FEE, pH-value, resistivity and temperature of the water in the standoff box are carefully monitored.

The average $\theta_{c}$ resolution for a single photon, as measured from the difference between measured and expected angle for reconstructed tracks from $\mu^{+} \mu^{-}$events, is about $10 \mathrm{mrad}$. Measured number of photoelectrons vary between 20 and 65 for small polar and large polar angles, yielding an overall $2.5 \mathrm{mrad}$ resolution for single muon tracks. Using this information and the difference between expected Cherenkov angles 
of pions and kaons, $4.2 \sigma \mathrm{K} / \pi$ separation is expected at $3 \mathrm{GeV} / c$ momentum. When combined with the energy loss information from the tracking system, the mean kaon selection efficiency can be as high as $96 \%$ for a pion misidentification rate of $2 \%$, as determined from kinematically identified $D^{0} \rightarrow K^{-} \pi^{+}$decays.

\subsection{Electromagnetic Calorimeter}

Located outside the DIRC, the electromagnetic calorimeter (EMC) is the component of the detector essential for the analysis described in this thesis. With excellent efficiency, energy and position resolution over a wide energy range from $20 \mathrm{MeV}$ to $9 \mathrm{GeV}$, it not only allows the identification and measurement of 2-3 GeV photon showers from the $B \rightarrow X_{s} \gamma$ process, but also the much lower energy showers from the decays of $\pi^{0}$ and $\eta$ mesons, allowing reconstruction, analysis and rejection of these main sources of background. Moreover, by identifying electrons, the EMC contributes to the lepton tagging of $B$ mesons for continuum background suppression and measurement of $C P$-violating asymmetry.

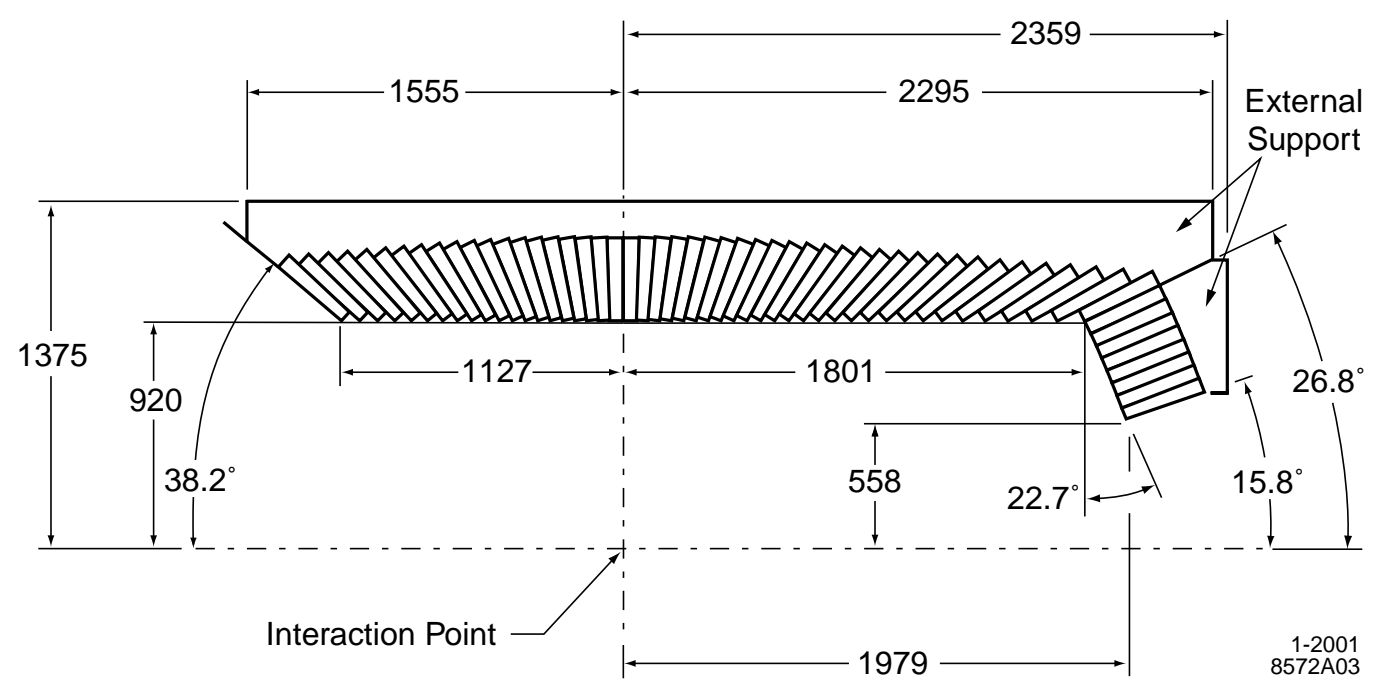

Figure 2.6: Top half of the longitudinal section of the electromagnetic calorimeter 33. Dimensions are given in millimeters.

The EMC is a hermetic, finely segmented, total-absorption calorimeter, composed of 6580 thallium-doped cesium iodide ( $\mathrm{CsI}(\mathrm{Tl})$ ) crystals, organized into a cylindrical 
barrel and a conical forward endcap (Figure 2.6). The barrel contains 5760 crystals arranged in 48 distinct rings with 120 crystals each. The endcap consists of eight rings: three rings of 120 , three rings of 100 and two rings of 80 crystals. In the c.m. system, $90 \%$ of the solid-angle is covered.

The crystals have a tapered trapezoidal longitudinal cross-section. Their transverse dimensions, comparable to the Molière radius of CsI $(3.8 \mathrm{~cm}[38)$, vary for each of the 56 rings, in order to achieve hermeticity. The typical area of the front face is $4.7 \times 4.7 \mathrm{~cm}^{2}$ and that of the back face is $6.1 \times 6.0 \mathrm{~cm}^{2}$. The length increases from 16.0 to 17.5 radiation lengths $\left(X_{0}\right)$ as the polar angle decreases, so as to limit the shower leakage for increasingly higher energy particles.

An important feature of the $B A B A R$ detector, mentioned briefly in the earlier sections, is the choice of materials and structural design to keep multiple scattering and pre-showering to a minimum level. In this spirit, the EMC crystals are supported at the outer radius. A particle from the IP encounters $0.3-0.6 X_{0}$ of material to reach the EMC. (The only exception is the three innermost rings of the endcap, which are shadowed by the SVT support structure and FEE and by the B1 dipole magnet. The principle purpose of these rings is to enhance shower containment for particles close to the acceptance limit.)

The CsI(Tl) scintillation light is read out by two silicon photodiodes connected to the back face of each crystal. The signal from each diode, on the order of 5000 photoelectrons $/ \mathrm{MeV}$, is amplified by a low-noise pre-amplifier, before being transferred to the on-detector FEE. Here a custom IC decides which of the signals from the two diode-preamplifier pairs to further process, or whether to take an average of the two. The signal is digitized by a 10-bit ADC in four different ranges of energy, dynamically spanning 0-13 GeV with no significant impact on the resolution.

Regular calibrations are essential to the operation, since the light yield shows about $12 \%$ RMS variation among crystals and is time-dependent (degrades slowly due to radiation damage). The electronics are calibrated with injected charge to the preamplifier input. At opposite ends of the energy range, two techniques are used to relate the pulse height in each crystal to the actual deposited energy: A $6.13 \mathrm{MeV}$ radioactive source provides the absolute calibration and the resolution $((5.0 \pm 0.8) \%)$ 
at the low energy, whereas at high end of the spectrum, the electrons from Bhabha scattering are used. In addition, the light yield is regularly monitored by a light-pulser system.

Typical electromagnetic showers spread over many crystals; on average 25 crystals are showered by the photons from the $B \rightarrow X_{s} \gamma$ decay. Such clusters are identified by the presence of at least one seed crystal with an energy deposit above $10 \mathrm{MeV}$ surrounded by crystals that are measuring at least $1 \mathrm{MeV}$ or neighboring (including corners) others with at least $3 \mathrm{MeV}$. Occasionally two or more particles are close enough to each other that their showers merge into one cluster. For this reason, once identified, every cluster is searched for local energy maxima. A pattern recognition algorithm then distributes a fraction of the energy from each crystal among multiple showers. A center-of-gravity method with logarithmic weights calculates the positions (centroids) of the showers, and a track extrapolation algorithm matches them to tracks from the drift chamber.

Once reconstructed, shower energies are additionally calibrated to account for the losses due the leakage out of the crystals and the absorption in the inter-crystal material. Corrections are derived from mass measurements in $\pi^{0} \rightarrow \gamma \gamma$ decays, from radiative Bhabha events and from single-photon Monte Carlo simulations.
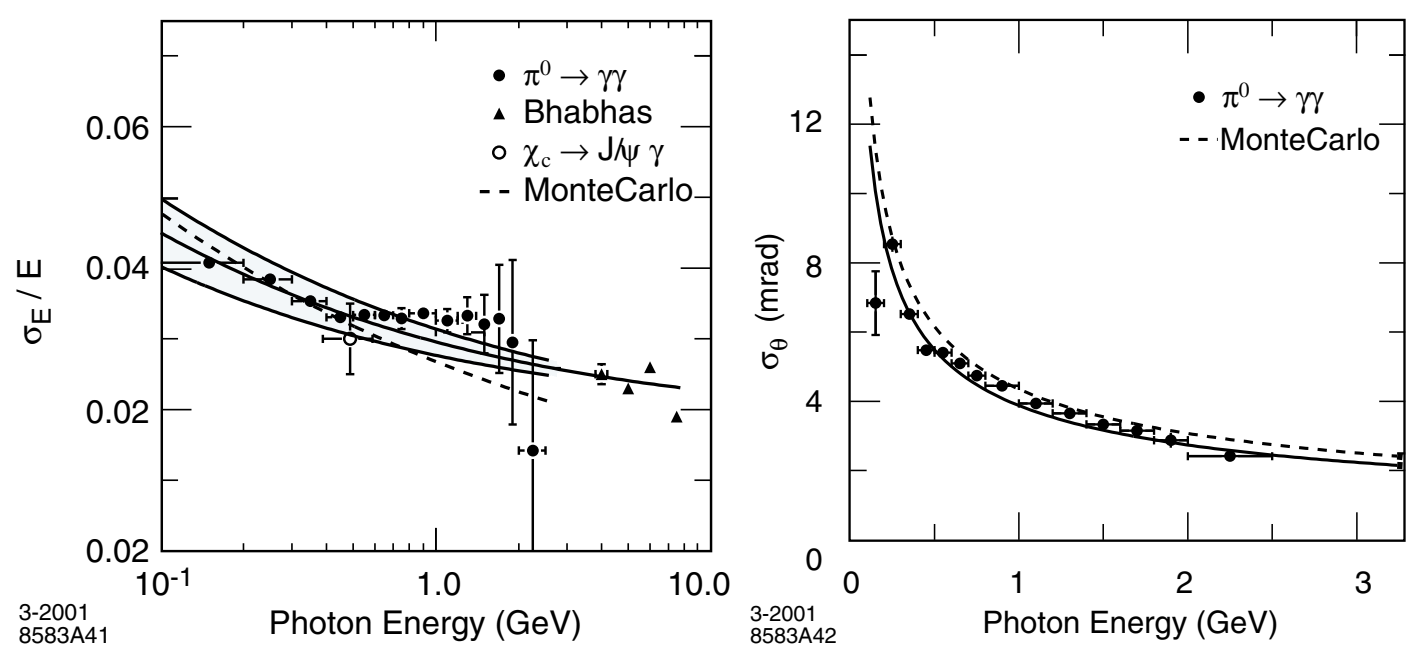

Figure 2.7: The energy and angular resolution for electromagnetic showers in the EMC, measured from various processes. 33. 
The energy resolution of the calorimeter is determined from various processes. The measurements from the radioactive source and from the Bhabha scattering are supplemented by the measurement of the photon energy from $\chi_{c 1} \rightarrow J / \psi \gamma$ decays and the measurements of the mass resolution of $\pi^{0}$ and $\eta$ mesons decaying symmetrically, i.e. into two photons of approximately equal energy. The results are plotted in Figure 2.7, where the solid curve is a fit to the function:

$$
\frac{\sigma_{E}}{E}=\frac{(2.32 \pm 0.30) \%}{\sqrt[4]{E(\mathrm{GeV})}} \oplus(1.85 \pm 0.12) \%
$$

Symmetrically-decaying $\pi^{0}$ and $\eta$ mesons are also useful for the determination of the angular resolution. The result, presented in Figure 2.7, varies between approximately $12 \mathrm{mrad}$ at low energies and $3 \mathrm{mrad}$ at high energies. A fit to an empirical parametrization of the energy dependence yields:

$$
\sigma_{\theta, \phi}=\left(\frac{3.87 \pm 0.07}{\sqrt{E(\mathrm{GeV})}}+0.00 \pm 0.04\right) \mathrm{mrad}
$$

Finally, another important measure of the calorimeter performance is the identification of electrons. The identification efficiency is measured using $e^{+} e^{-} \rightarrow e^{+} e^{-} \gamma$ and $e^{+} e^{-} \rightarrow e^{+} e^{-} e^{+} e^{-}$events. For the selection criteria used in the $B \rightarrow X_{s} \gamma$ analysis, this efficiency is as high as $90 \%$, with less than $0.2 \%$ misidentification probability for pions (Figure 2.8).
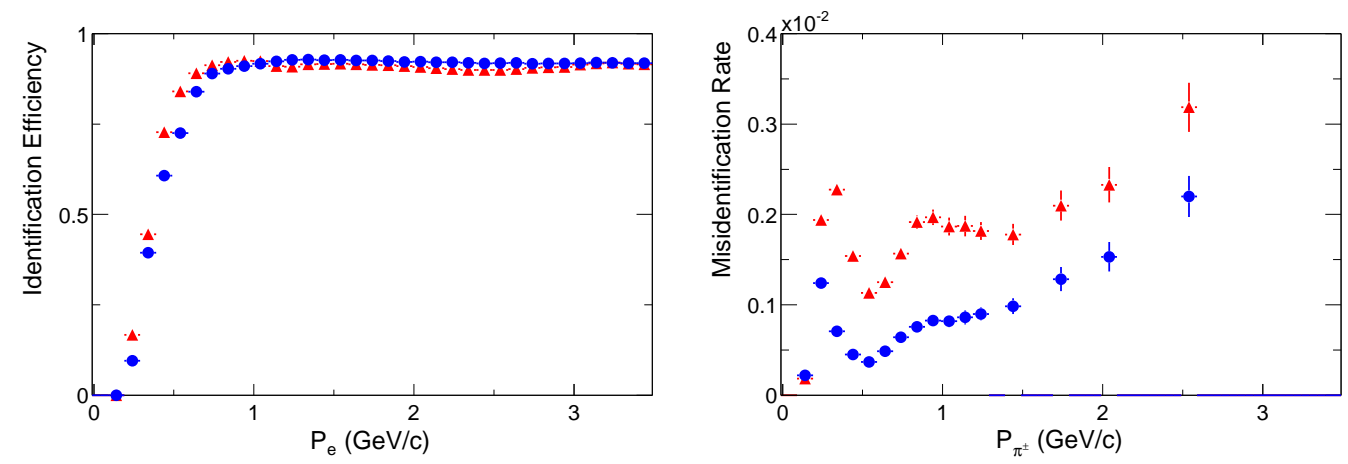

Figure 2.8: Electron identification efficiency (left) and misidentication probability of pions as electrons (right), as a function of the momentum of the particle. Blue circles (red triangles) indicate negatively(positively)-charged particles. 


\subsection{Instrumented Flux Return}

Designed to identify muons and neutral hadrons with good efficiency and high background rejection, the Instrumented Flux Return (IFR) is the outermost part of the $B A B A R$ detector. It is composed of resistive plate chambers (RPCs) installed in the gaps between the 18 plates of the steel flux return (Figure 2.9). Additionally, between the EMC and the magnet cryostat are two extra layers of cylindrical RPCs, to detect particles exiting the calorimeter.

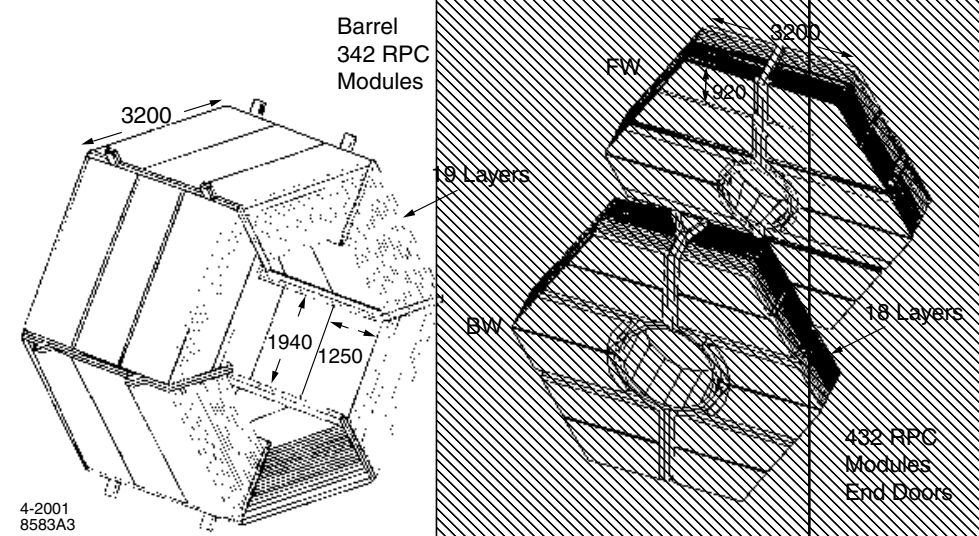

Figure 2.9: An orthographic projection of the barrel and endcap sections of the IFR [33. The system consists of a total of 806 RPC modules, whose shape and size conform to the mechanical partitioning of the steel flux return, with very little dead space. Dimensions are given in millimeters.

As illustrated in Figure 2.10, each RPC layer consists of two bakelite sheets kept $2 \mathrm{~mm}$ apart by $0.8 \mathrm{~cm}^{2}$ polycarbonate spacers positioned about every $10 \mathrm{~cm}$. External surfaces of these sheets are coated with graphite and a $7.6 \mathrm{kV}$ potential is applied to one of them, while the other is grounded. On the outside of the graphite coating, insulated by mylar films, are $2-4 \mathrm{~cm}$ - wide aluminum strips

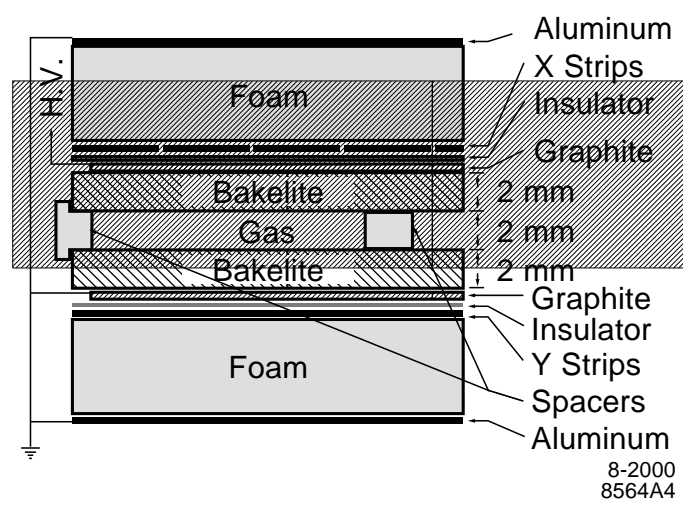

Figure 2.10: Cross-section of a planar RPC with the schematics of the high voltage connection [39]. 
capacitively reading-out the streamer signals from ionizing particles. The orientation of the strips on the two sides of a chamber is orthogonal. This allows three-dimensional reconstruction in the IFR and the association of signals to tracks reconstructed in the DCH or to neutral clusters from the EMC.

In total, there are close to 53,000 strips in the IFR. These channels are serviced by 3300 front-end cards, each of which shapes 16 input signals and sets a bit for those above a fixed threshold. Additionally, a fast OR of the 16 signals is used by TDC boards to extract time information.

Discrimination of muons from charged hadrons relies on a number of quantities measured in the IFR. These include the total number of interaction lengths traversed from the IP to the outermost associated IFR hit, the mean and the variance of the number of hit strips per layer, and the $\chi^{2}$ for the geometric match between a projected track and the position of the hits. At the time of commissioning, $80 \%$ of the 1.5 -to$3.0 \mathrm{GeV} / c$ muons from $\mu \mu \gamma$ and $\mu \mu e e$ final states could be identified correctly for a certain set of tight selection criteria.

Unfortunately, the average RPC efficiency has declined steadily since 1999. The initial efficiency of approximately 85\%, had decreased to about $65 \%$ by June 2002 . Various reasons have been identified for the observed degradation, primarily the overheating of the RPCs in the summer of 2000 and problems with the linseed oil that was used to smooth the gap-side surfaces of the bakalite electrodes [40].
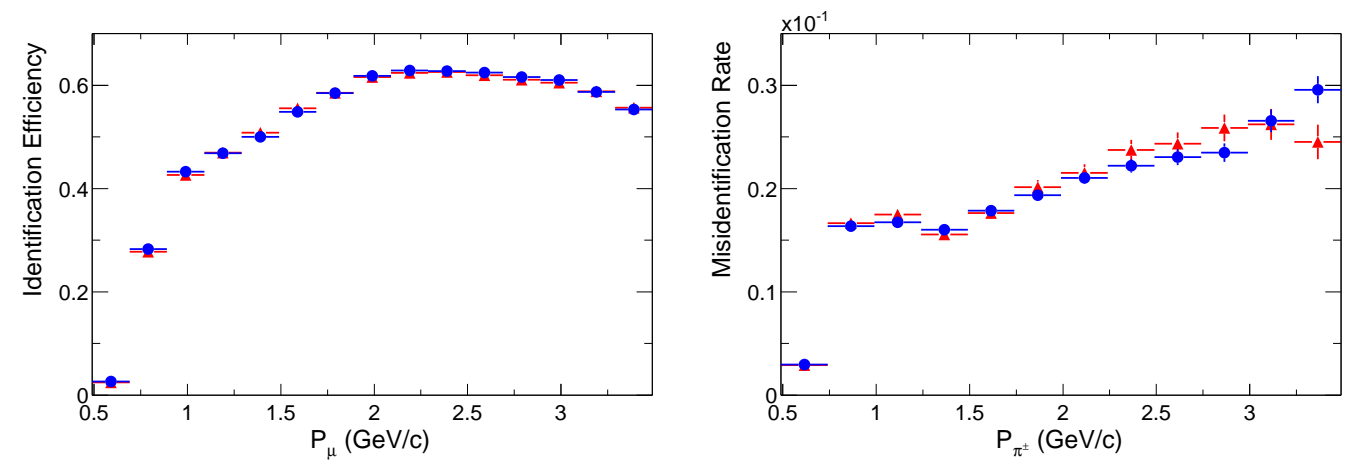

Figure 2.11: Muon identification efficiency (left) and misidentication probability of pions as muons (right), as a function of the momentum of the particle, averaged over the data sample used for this thesis. Blue circles (red triangles) indicate negatively(positively)-charged particles. 


\subsection{The BABAR Trigger}

At the design luminosity of $3 \times 10^{33} \mathrm{~cm}^{-2} \mathrm{~s}^{-1}$, PEP-II delivers $B \bar{B}$ events at a rate of about $3 \mathrm{~Hz}$. It also delivers a similar rate of $\tau^{+} \tau^{-}$and $\mu^{+} \mu^{-}$events, and three times more $q \bar{q}$ events, making it a $\tau^{ \pm}$and charm factory as well as a $B$ Factory. However, there beam backgrounds contribute another $20 \mathrm{KHz}$ of events that have to be rejected so that the event rate is at a manageable level for data acquisition and storage. This task is performed by a two-level trigger system, which reduces the total event rate to about $100 \mathrm{~Hz}$, while keeping essentially all the events of interest.

\subsubsection{Level 1 Trigger System}

The first level of the trigger system, the Level 1 (L1) trigger, is implemented in hardware. Three specialized processors, one for the DCH, one for the EMC and a third one for the IFR, independently process the data from their respective detector systems to generate trigger primitives, i.e. summary data that is subsequently processed by the global trigger (GLT), before a $L 1$ Accept is issued to initiate event readout.

One bit for each of the 7104 DCH cells, sampled every 269 ns, are the inputs to the drift chamber trigger (DCT). Using a look-up-table, each group of eight contiguous cells spanning all four layers of a superlayer is first tested for possible straight-line track segments. The identified segments are then mapped onto the DCH geometry in terms of 320 supercells, 10 radial superlayers each divided into 32 sectors in $\phi$. With this map, moving radially outwards from the innermost superlayer, segments are connected into short(long) track candidates, reaching superlayer 5(10). In parallel, the same track segments are independently checked for high-transverse-momentum track candidates $\left(p_{T} \gtrsim 0.8 \mathrm{GeV} / c\right)$ starting from superlayers 7 and 10 moving in an azimuthal wedge towards the IP. Finally DCT passes three types of trigger primitives to the GLT: 16-bit $\phi$-maps for identified short, long or high- $p_{T}$ track candidates.

The inputs to the EMT are the sums of all crystal energies above a threshold of $20 \mathrm{MeV}$ calculated in 280 EMC towers, defined as $8 \times 3(\theta \times \phi)$ arrays of crystals in the barrel, and azimuthal wedges of 19-22 crystals in the endcap. The EMT handles these inputs in $40 \phi$-sectors, summing up energies of certain towers in each sector 
and comparing with adjustable thresholds. The output is in the form of five types of trigger primitives with 20-bit $\phi$-maps.

The DCT and EMT independently satisfy all the trigger requirements with high efficiency, thereby providing a high degree of redundancy. The instrumented flux return trigger (IFT) on the other hand, is mainly used for $\mu^{+} \mu^{-}$and cosmic ray events. The IFR is partitioned into ten sectors, and data from selected layers of each sector is used for the trigger decision. IFT is mostly for diagnostic purposes.

GLT combines the trigger primitives from the DCT, EMT and IFT into 24 trigger lines and delivers them to a Fast Control and Timing System, which can optionally mask or prescale any of these. If a valid trigger remains after this step, the L1 Accept signal is issued to the detector components. Between the sending of the raw data to the $\mathrm{L} 1$ trigger and the initiation of the event readout just about $10 \mu \mathrm{s}$ is taken, within the limits of $12.8 \mu$ s latency buffers installed on the FEE circuitry of all the detector components.

\subsubsection{Level 3 Trigger System}

L1 Accept is issued at a typical rate of $1 \mathrm{KHz}$. At this rate, access to complete event information is possible and the Level 3 (L3) trigger can be implemented as software running on an online computer farm. Its task is to improve the rejection of beam backgrounds and to scale down the rate of Bhabha events.

Many of the beam-induced background events from the L1 are due to charged particles produced in material close to the IP. For instance, a flange of the beam pipe is the cause of many L1 tracks with $\left|z_{0}\right|$ close to $20 \mathrm{~cm}$. In order to reject these events, L3 drift chamber algorithm performs a fast pattern recognition followed by track fitting. Track segments from L1 are searched in a look-up-table populated with hit parameters from Monte Carlo generated tracks with $p_{T}>250 \mathrm{MeV} / c$ that originate within $2 \mathrm{~cm}$ of the IP in the $\mathrm{x}-\mathrm{y}$ plane, and within $10 \mathrm{~cm}$ along the $\mathrm{z}$. Matching segments and their associated hits are then fit by an iterative algorithm, which drops hits with high residuals and adds new segments as necessary. With this algorithm, the achieved $z_{0}$ resolution is better than $1 \mathrm{~cm}$, significantly improving the performance of 
the following L3 filters.

For the identification and rejection of Bhabha events, DCH data must be complemented with precise measurements of the calorimeter showers. To achieve this and to further reduce the background events passing the EMT, the L3 EMC-based trigger selects individual crystal signals within a $1.3 \mu$ s window around the event time. Employing a fast look-up-table, it reconstructs clusters out of these signals. Clusters with an energy above $100 \mathrm{MeV}$ are retained, and centroid position, average time and lateral shower profile for particle identification are calculated.

The combined effiency of the L1 and L3 triggers is better than $99.9 \%$ for generic $B \bar{B}$ events. About $97 \%$ of $q \bar{q}$ and $92 \%$ of $\tau^{+} \tau^{-}$events are also retained. Additionally, prescaled samples of (radiative) Bhabha, two-photon, cosmic ray and random trigger events are also accepted for purposes of calibration, diagnostics and offline luminosity measurement.

\subsection{Data Sets and Simulation}

Since its commissioning, the BABAR detector has collected about $300 \mathrm{fb}^{-1}$ of data. In this analysis, only $91 \mathrm{fb}^{-1}$ of this data, collected between March 2000 and June 2002, are used. As mentioned in section [1.9, the extraction of the $B \rightarrow X_{s} \gamma$ signal requires a detailed study of the detector response and the backgrounds with Monte Carlo (MC) simulation, and the precision tuning of the Monte Carlo samples have yet been performed for this part of the data sample.

The production of the MC samples is performed in two steps. The first step is the event generation, where a phenomenological simulation is carried out for the physics processes of interest starting with the initial $e^{+} e^{-}$pair. For different final states, different simulation packages are used. Bhabha events are generated by BHWIDE [41, $\tau^{+} \tau^{-}$events by KoralB [42], and $B \bar{B}$ and $q \bar{q}$ events using EvtGen [43] and Jetset [44]. In EvtGen, QED radiative effects are simulated by PHOTOS for final states with electrons or muons [45].

In the second step, GEANT4 package is used to propogate the particles from the generators inside the detector [46]. Energy loss in the detector, multiple scattering, 
Bremsstrahlung are some of the interactions simulated. The detector hits from GEANT are then converted to objects that mimic the electronic output of the detector, and mixed with corresponding real output from the background events accepted on random trigger signals. Finally, regular L1 and L3 trigger requirements are applied and event reconstruction is performed.

For each Monte Carlo event, the output from both steps of the production are retained in the event database. This allows the recovery of true values of the reconstructed quantities, for studies of detector resolution and inefficiencies. The procedure of connecting these true and reconstructed values is referred to as MC truth matching. It is performed by comparing the total number (energy) of shared tracking-system (calorimeter) hits between the true and reconstructed charged (neutral) particle candidates in each simulated event. It is remarkably accurate, especially for high energy particles with many detector hits [47].

\begin{tabular}{|l|r|r|c|}
\hline Data Set & Events & Cross-Section [4] or $\mathcal{B}$ & Luminosity \\
\hline \hline$u \bar{u}, d \bar{d}, s \bar{s}$ & 124230000 & $2.09 \mathrm{nb}$ & $59.4 \mathrm{fb}^{-1}$ \\
$c \bar{c}$ & 61417500 & $1.30 \mathrm{nb}$ & $47.2 \mathrm{fb}^{-1}$ \\
$\tau^{+} \tau^{-}$ & 66264000 & $0.94 \mathrm{nb}$ & $70.5 \mathrm{fb}^{-1}$ \\
$B^{0} \bar{B}^{0}$ & 101278351 & $0.543 \mathrm{nb}$ & $186.5 \mathrm{fb}^{-1}$ \\
$B^{+} B^{-}$ & 102989673 & $0.543 \mathrm{nb}$ & $189.7 \mathrm{fb}^{-1}$ \\
\hline$B^{0} \rightarrow K^{* 0}(892) \gamma$ & 111000 & $3.90 \pm 0.43 \times 10^{-5}$ & $2621 \mathrm{fb}^{-1}$ \\
$B^{ \pm} \rightarrow K^{* \pm}(892) \gamma$ & 117000 & $3.90 \pm 0.43 \times 10^{-5}$ & $2762 \mathrm{fb}^{-1}$ \\
$B^{0} \rightarrow X_{s}^{0} \gamma$ (KN480) & 83992 & $2.18 \pm 0.24 \times 10^{-4}$ & $354.8 \mathrm{fb}^{-1}$ \\
$B^{ \pm} \rightarrow X_{s}^{ \pm} \gamma$ (KN480) & 80829 & $2.18 \pm 0.24 \times 10^{-4}$ & $341.4 \mathrm{fb}^{-1}$ \\
$B^{0} \rightarrow X_{s}^{0} \gamma$ (flat) & 513999 & & \\
$B^{ \pm} \rightarrow X_{s}^{ \pm} \gamma$ (flat) & 615867 & & \\
\hline On-Resonance & \multicolumn{3}{|c|}{$n_{B \bar{B}}=88477212 \pm 1.1 \%$} \\
Off-Resonance & & $81.5 \pm 1.2 \mathrm{fb}^{-1}$ \\
\hline
\end{tabular}

Table 2.1: Monte Carlo and real data sets. The $B \rightarrow X_{s} \gamma$ samples exclude $B \rightarrow K^{*} \gamma$ events and have no events in which the photon energy exceeds $2.525 \mathrm{GeV}$, in accordance with the Kagan and Neubert prescription (Section (1.8).

Table 2.1 lists the major data sets (both real and simulated) used in the $B \rightarrow X_{s} \gamma$ analysis. Other samples have also been used for various purposes; for instance Bhabha 
samples were used for precise determination of tracking inefficiencies, etc. For each type of data set, its size and the corresponding integrated luminosity are tabulated.

The integrated luminosity for the MC samples are calculated from the number of events and the cross-section for the relevant process. The integrated luminosity for the real data samples are measured by counting the $\mu^{+} \mu^{-}$events in each sample and correcting this count for detection efficiency. This value is validated by a similar studies with (radiative) Bhabha and $\gamma \gamma$ events [49].

Additionally, for the on-resonance data sample, the total number of $B \bar{B}$ pairs, $n_{B \bar{B}}$, is determined [50]. In the off-resonance sample, the ratio of the number of multihadronic events to the number of $\mu^{+} \mu^{-}$events is measured. This ratio is then used with the number of $\mu^{+} \mu^{-}$events in the on-resonance sample to calculate the number of non- $B \bar{B}$ multihadronic events, which is subtracted from the total number of multihadronic events in the on-resonance sample to obtain $n_{B \bar{B}}$ [51].

Three types of signal Monte Carlo samples are used. The first type consists of $B \bar{B}$ events in which at least one of the $B$-mesons exlusively decays in the $B \rightarrow K^{*} \gamma$ channel. The other two types of simulated signal samples consist of $B \rightarrow X_{s} \gamma$ events with photons constrained to have energies below $2.525 \mathrm{GeV}$ in the rest frame of the $B$-meson. (The value of this cutoff has been selected to conform with the Kagan and Neubert prescription discussed in Section [1.8) One sample of $B \rightarrow X_{s} \gamma$ events is generated such that the resulting photon energy spectrum is given by the KN480 model. The other sample of $B \rightarrow X_{s} \gamma$ events is generated such that the final photon energy distribution is flat. By simply assigning weights to each event in this sample, the flat distribution of photon energies can be shaped into any arbitrary distribution predicted by various signal models. This weighting is done in $E_{\gamma}$ using look-up tables with $13 \mathrm{MeV}$ granularity for KN models and $10 \mathrm{MeV}$ granularity for BBU and LNP models. 


\section{Chapter 3}

\section{Event Selection}

The selection of events is the first stage of the analysis. The goal for this stage is to identify a set of criteria that rejects background processes while retaining as many $B \rightarrow X_{s} \gamma$ signal events as possible. Various distinguishing features of the signal are studied using Monte Carlo simulation to be later looked for in the data.

Some of those features, like the presence of a high momentum lepton discussed in Section 3.7. are very powerful in rejecting the backgrounds, but are present only in a subset of the signal events. Therefore a figure of merit, based on the expected statistical significance of the total inclusive branching fraction, is defined to quantify the performance of the selection criteria. An optimization process, detailed in Section 3.8, has been carried out to maximize this figure of merit.

In order not to introduce any experimenter bias, it is essential that the identification and the optimization of the selection criteria be done with Monte Carlo samples only. This blind approach of keeping the signal regions of the data hidden is carried out until the analysis is essentially complete. Obviously, this adds another level of complication to the choice of the criteria: The selection has to be robust against possible systematic differences between data and simulation. 


\subsection{Selection of High-Quality Particle Candidates}

Before the actual selection of events, each event is scanned for high-quality charged particle and photon candidates. Every track and neutral shower, i.e. a shower not associated to any track, satisfying the criteria listed in Table 3.1 is flagged as a good track or a good photon.

\begin{tabular}{|l|l|l|}
\hline Particle & Parameter & Criterion \\
\hline Good track & Transverse momentum & $p_{T}>0.1 \mathrm{GeV} / c$ \\
& Momentum & $p<10 \mathrm{GeV} / c$ \\
& Number of DCH hits & $n_{D C H}>11$ \\
& DOCA to IP in $x y$ plane & $d_{0}^{I P}<1.5 \mathrm{~cm}$ \\
& DOCA to IP along $z$ & $\left|z_{0}^{I P}\right|<10 \mathrm{~cm}$ \\
\hline Good photon & Energy & $E>30 \mathrm{MeV}$ \\
& Lateral moment & $L A T<0.8$ \\
\hline
\end{tabular}

Table 3.1: The criteria satisfied by tracks and neutral showers to be identied as good tracks and good photons. DOCA stands for distance of closest approach. Lateral moment is defined in section 3.3.2.

The main purpose of this selection is to improve the studies of event topology, which rely on the distribution all particles inside the detector, by reducing the number of tracks and showers from cosmic rays, from SVT or calorimeter noise, from lowenergy interactions of hadrons, etc. Their effect on particles from physics events of interest are negligible. About $97 \%$ of all charged particles originating from the IP leave good tracks [52]. Of the electromagnetic showers that satisfy the $30 \mathrm{MeV}$-energy criteria, virtually all are flagged as good photons, whereas about $10 \%$ of the showers from hadronic interactions in the EMC are rejected [53].

\subsection{Event Preselection}

The event selection starts by the basic requirement that a photon candidate in the right energy range be identified by the calorimeter. For this purpose, a good photon with c.m. energy of $1.5<E_{\gamma}^{*}<3.5 \mathrm{GeV}$ is searched for. Such a candidate is present in approximately $78 \%$ of the signal events, with most of the remaining $22 \%$ lost when the 
signal photon falls outside the calorimeter acceptance or is mis-reconstructed. More than $97 \%$ of all other $B \bar{B}$ events, and about seven-eighths of $q \bar{q}$ events are rejected by this requirement.

The search for the photon candidate does not require any significant processing power, hence choosing it as a preselection criterion has the added advantage of reducing the computational load of the full analysis chain. To further increase the computational benefit, three other preselection requirements are imposed: that there be at least three good tracks in the event, that the normalized second Fox-Wolfram moment calculated in the c.m. frame using tracks be less than 0.9 , and that no track have more than $2.5 \mathrm{GeV} / \mathrm{c}$ momentum in the c.m. frame. These are meant mainly to reduce QED continuum events, which tend to have low track multiplicity, with tracks of high momentum from the IP emitted back-to-back in the c.m.frame. The limit on the highest track momentum provides some reduction of $q \bar{q}$ backgrounds as well, especially for light quarks.

The photon spectrum after the preselection is plotted in Figure 1.3.

\subsection{Selection of the Signal Photon}

After the preselection, the event selection proceeds in three steps. The first step rejects events based on criteria applied to the high-energy photon candidates, which have been identified during preselection. In this step, when multiple candidates exist in an event (2.5\% of the preselected events), the candidates are independently subjected to the requirements. An event is discarded if no candidates satisfy the criteria.

\subsubsection{Photon Quality Criteria}

To improve the accuracy of the measurement and to avoid error due to noise, the photon candidates are initially required to satisfy the following quality criteria:

- Fiducial polar acceptance. To ensure that the shower is well-contained in the EMC, the centroid of the candidate is required to be about 4 (1.5) crystal widths away from the forward (backward) edge of the calorimeter. This constraint also 


\begin{tabular}{|c|c|c|c|c|c|}
\hline \multirow[b]{2}{*}{ Sec. } & \multirow[b]{2}{*}{ Description } & \multicolumn{4}{|c|}{ Signal } \\
\hline & & $X_{s}^{0} \gamma$ & $X_{s}^{+} \gamma$ & $K^{* 0} \gamma$ & $K^{*+} \gamma$ \\
\hline$\overline{33.2}$ & "Photon with $1.5 \mathrm{GeV}<E_{\gamma}^{*}<3.5 \mathrm{GeV}$ & $\overline{0.78}$ & 0.78 & 0.79 & 0.79 \\
\hline \multirow[t]{2}{*}{3.2} & QED continuum rejection & 0.94 & 0.95 & 0.92 & 0.93 \\
\hline & Cumulative Preselection & 0.736 & 0.743 & 0.720 & 0.731 \\
\hline 3.3 .1 & $-0.74<\cos \theta_{\gamma}<0.93$ & $\overline{0.97}$ & 0.97 & 0.97 & $\overline{0.97}$ \\
\hline 3.3 .1 & No problematic channels & 0.98 & 0.98 & 0.98 & 0.98 \\
\hline 3.3 .1 & $\mathrm{n}_{\text {Crystals }} \geq 4$ & 1.00 & 1.00 & 1.00 & 1.00 \\
\hline 3.3 .2 & Second Moment $<0.002 \mathrm{rad}^{2}$ & 0.98 & 0.98 & 0.98 & 0.98 \\
\hline 3.3 .2 & $L A T<0.45$ & 1.00 & 1.00 & 1.00 & 1.00 \\
\hline 3.3 .2 & Bump Isolation $>25 \mathrm{~cm}$ & 0.91 & 0.90 & 0.91 & 0.91 \\
\hline 3.3 .3 & $\pi^{0}$ veto & 0.94 & 0.94 & 0.97 & 0.97 \\
\hline \multirow[t]{2}{*}{3.3 .3} & $\eta$ veto & 0.94 & 0.94 & 0.98 & 0.98 \\
\hline & Cumulative Photon Selection & 0.74 & 0.74 & 0.80 & 0.80 \\
\hline 3.4 & $22.0 \mathrm{GeV}<E_{\gamma}^{*}<2.7 \mathrm{GeV}$ & $\overline{0.91}$ & $\overline{0.91}$ & $\overline{0.92}$ & $\overline{0.91}$ \\
\hline 3.5 & 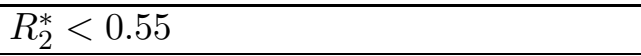 & 0.97 & 0.97 & 0.92 & 0.94 \\
\hline \multirow[t]{2}{*}{3.5} & Fisher Discriminant $>0.575$ & 0.61 & 0.59 & 0.80 & 0.75 \\
\hline & Cumulative Event Shape & 0.59 & 0.57 & 0.74 & 0.70 \\
\hline \multirow[t]{2}{*}{3.6} & Single Photon Candidate & 1.00 & 1.00 & 1.00 & 1.00 \\
\hline & Cumulative before Tag & 0.291 & 0.284 & 0.393 & 0.373 \\
\hline 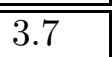 & Electron Tag $p_{e}^{*}>1.25 \mathrm{GeV} / c$ & 0.039 & 0.041 & 0.042 & 0.044 \\
\hline 3.7 & $\cos \theta_{\gamma e}>-0.7$ & 0.874 & 0.879 & 0.904 & 0.911 \\
\hline 3.7 & Muon Tag $p_{\mu}^{*}>1.5 \mathrm{GeV} / c$ & 0.021 & 0.022 & 0.025 & 0.024 \\
\hline 3.7 & $\cos \theta_{\gamma \mu}>-0.7$ & 0.804 & 0.795 & 0.794 & 0.826 \\
\hline \multirow[t]{2}{*}{3.7} & $E_{\text {miss }}^{*}>0.8 \mathrm{GeV}$ & 0.972 & 0.969 & 0.941 & 0.944 \\
\hline & Cumulative Lepton Tag & 0.050 & 0.052 & 0.054 & 0.056 \\
\hline \multirow[t]{2}{*}{ 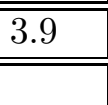 } & $\overline{N_{\text {mult }} \geq 4.5}$ & 0.995 & 0.998 & 0.990 & 0.993 \\
\hline & TOTAL Efficiency & 0.0144 & 0.0146 & 0.0212 & 0.0208 \\
\hline
\end{tabular}

Table 3.2: Selection efficiencies for $B \rightarrow X_{s} \gamma$ (from Kagan-Neubert model with $m_{b}=4.80 \mathrm{GeV} / c^{2}$ ) and $B \rightarrow K^{*} \gamma$, from signal Monte Carlo samples. Efficiency for each criterion is given in the order it is applied. 


\begin{tabular}{|c|c|c|c|c|}
\hline \multirow[b]{2}{*}{ Sec. } & \multirow[b]{2}{*}{ Description } & \multicolumn{3}{|c|}{$q \bar{q}$ background } \\
\hline & & $\pi^{0} / \eta$ & ISR & other \\
\hline 3.2 & Photon with $1.5<E_{\gamma}^{*}<3.5 \mathrm{GeV}$ & \multicolumn{3}{|c|}{0.124} \\
\hline \multirow[t]{2}{*}{3.2} & QED continuum rejection & \multicolumn{3}{|c|}{0.799} \\
\hline & Cumulative Preselection & \multicolumn{3}{|c|}{0.099} \\
\hline 3.3 .1 & $-0.74<\cos \theta_{\gamma}<0.93$ & 0.96 & 0.91 & 0.95 \\
\hline 3.3 .1 & No problematic channels & 0.97 & 0.98 & 0.97 \\
\hline 3.3 .1 & $\mathrm{n}_{\text {Crystals }} \geq 4$ & 1.00 & 1.00 & 1.00 \\
\hline 3.3 .2 & Second Moment $<0.002 \mathrm{rad}^{2}$ & 0.94 & 0.97 & 0.39 \\
\hline 3.3 .2 & $L A T<0.45$ & 0.99 & 1.00 & 0.91 \\
\hline 3.3 .2 & Bump Isolation $>25 \mathrm{~cm}$ & 0.46 & 0.93 & 0.58 \\
\hline 3.3 .3 & $\pi^{0}$ veto & 0.53 & 0.95 & 0.84 \\
\hline \multirow[t]{2}{*}{3.3 .3} & $\eta$ veto & 0.65 & 0.94 & 0.83 \\
\hline & Cumulative Photon Selection & 0.14 & 0.71 & 0.14 \\
\hline 3.4 & $22.0 \mathrm{GeV}<E_{\gamma}^{*}<2.7 \mathrm{GeV}$ & 0.31 & 0.36 & 0.30 \\
\hline 3.5 & " & 0.75 & 0.97 & 0.78 \\
\hline \multirow[t]{2}{*}{3.5} & Fisher Discriminant $>0.575$ & 0.25 & 0.15 & 0.23 \\
\hline & Cumulative Event Shape & 0.19 & 0.15 & 0.18 \\
\hline \multirow[t]{3}{*}{3.6} & Single Photon Candidate & 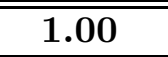 & 1.00 & 0.99 \\
\hline & \multirow{2}{*}{ Expected Events, $80.5 \mathrm{fb}^{-1}$} & 173075 & 95670 & 32073 \\
\hline & & \multicolumn{3}{|c|}{300818} \\
\hline 3.7 & Electron Tag $p_{e}^{*}>1.25 \mathrm{GeV} / c$ & 0.0053 & 0.0049 & 0.0047 \\
\hline 3.7 & $\cos \theta_{\gamma e}>-0.7$ & 0.0553 & 0.2188 & 0.1030 \\
\hline 3.7 & Muon Tag $p_{\mu}^{*}>1.5 \mathrm{GeV} / c$ & 0.0063 & 0.0045 & 0.0062 \\
\hline 3.7 & $\cos \theta_{\gamma \mu}>-0.7$ & 0.0307 & 0.1180 & 0.0576 \\
\hline \multirow[t]{2}{*}{3.7} & $E_{\text {miss }}^{*}>0.8 \mathrm{GeV}$ & 0.7664 & 0.8867 & 0.6876 \\
\hline & Cumulative Lepton Tag & 0.00037 & 0.00142 & 0.00058 \\
\hline \multirow[t]{5}{*}{3.9} & $N_{\text {mult }} \geq 4.5$ & 1.000 & 1.000 & 1.000 \\
\hline & \multirow{2}{*}{ TOTAL Efficiency } & $2.93 e-07$ & $5.26 \mathrm{e}-06$ & $4.13 \mathrm{e}-07$ \\
\hline & & \multicolumn{3}{|c|}{$8.01 \mathrm{e}-07$} \\
\hline & \multirow{2}{*}{ Expected Events, $80.5 \mathrm{fb}^{-1}$} & 65 & 135 & 19 \\
\hline & & \multicolumn{3}{|c|}{219} \\
\hline
\end{tabular}

Table 3.3: Selection efficiencies and expected number of events in $80.5 \mathrm{fb}^{-1}$ for $q \bar{q}$ continuum background, determined from Monte Carlo samples. The background is divided into its principal components $\left(\pi^{0} / \eta \rightarrow \gamma \gamma\right.$ decays, photons from initial-state radiation, and other). Efficiency for each criterion is given in the order it is applied. 


\begin{tabular}{|c|c|c|c|c|}
\hline \multirow[b]{2}{*}{ Sec. } & \multirow[b]{2}{*}{ Description } & \multicolumn{3}{|c|}{$\overline{\tau^{+} \tau^{-} \text {background }}$} \\
\hline & & $\pi^{0} / \eta$ & ISR & other \\
\hline 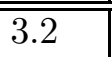 & "Photon with $1.5<E_{\gamma}^{*}<3.5 \mathrm{GeV}$ & \multicolumn{3}{|c|}{0.154} \\
\hline \multirow[t]{2}{*}{3.2} & QED continuum rejection & \multicolumn{3}{|c|}{0.167} \\
\hline & Cumulative Preselection & \multicolumn{3}{|c|}{0.026} \\
\hline 3.3 .1 & $-0.74<\cos \theta_{\gamma}<0.93$ & 0.96 & 0.91 & 0.88 \\
\hline 3.3 .1 & No problematic channels & 0.97 & 0.98 & 0.96 \\
\hline 3.3 .1 & $\mathrm{n}_{\text {Crystals }} \geq 4$ & 1.00 & 1.00 & 1.00 \\
\hline 3.3 .2 & Second Moment $<0.002 \mathrm{rad}^{2}$ & 0.97 & 0.99 & 0.57 \\
\hline 3.3 .2 & $L A T<0.45$ & 1.00 & 1.00 & 0.97 \\
\hline 3.3 .2 & Bump Isolation $>25 \mathrm{~cm}$ & 0.33 & 0.95 & 0.48 \\
\hline 3.3 .3 & $\pi^{0}$ veto & 0.49 & 0.97 & 0.93 \\
\hline \multirow[t]{2}{*}{3.3 .3} & $\eta$ veto & 0.95 & 0.98 & 0.97 \\
\hline & Cumulative Photon Selection & 0.14 & 0.80 & 0.20 \\
\hline 3.4 & $22.0 \mathrm{GeV}<E_{\gamma}^{*}<2.7 \mathrm{GeV}$ & $\overline{0.34}$ & 0.35 & $\overline{0.32}$ \\
\hline 3.5 & $\bar{R}_{2}^{*}<0.55$ & 0.05 & 0.81 & 0.07 \\
\hline \multirow[t]{2}{*}{3.5} & Fisher Discriminant $>0.575$ & 0.22 & 0.05 & 0.25 \\
\hline & Cumulative Event Shape & 0.01 & 0.04 & 0.02 \\
\hline \multirow[t]{3}{*}{3.6} & Single Photon Candidate & 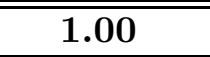 & 1.00 & 0.90 \\
\hline & \multirow{2}{*}{ Expected Events, $80.5 \mathrm{fb}^{-1}$} & 860 & 2375 & 168 \\
\hline & & \multicolumn{3}{|c|}{3403} \\
\hline 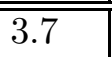 & Electron Tag $p_{e}^{*}>1.25 \mathrm{GeV} / c$ & 0.011 & $\overline{0.021}$ & 0.034 \\
\hline 3.7 & $\cos \theta_{\gamma e}>-0.7$ & 0.125 & 0.163 & 0.400 \\
\hline 3.7 & Muon Tag $p_{\mu}^{*}>1.5 \mathrm{GeV} / c$ & 0.007 & 0.013 & 0.007 \\
\hline 3.7 & $\cos \theta_{\gamma \mu}>-0.7$ & 0.000 & 0.111 & 1.000 \\
\hline \multirow[t]{2}{*}{3.7} & $E_{\text {miss }}^{*}>0.8 \mathrm{GeV}$ & 1.000 & 1.000 & 1.000 \\
\hline & Cumulative Lepton Tag & 0.0013 & 0.0048 & 0.0204 \\
\hline \multirow[t]{5}{*}{3.9} & $N_{\text {mult }} \geq 4.5$ & 0.000 & 0.800 & 1.000 \\
\hline & \multirow{2}{*}{ TOTAL Efficiency } & $<4.02 \mathrm{e}-08$ & $1.08 \mathrm{e}-06$ & $4.92 \mathrm{e}-07$ \\
\hline & & \multicolumn{3}{|c|}{$1.66 \mathrm{e}-07$} \\
\hline & \multirow{2}{*}{ Expected Events, $80.5 \mathrm{fb}^{-1}$} & 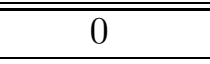 & 9 & 3 \\
\hline & & \multicolumn{3}{|c|}{13} \\
\hline
\end{tabular}

Table 3.4: Selection efficiencies and expected number of events in $80.5 \mathrm{fb}^{-1}$ for $\tau^{+} \tau^{-}$continuum background, determined from Monte Carlo samples. The background is divided into its principal components $\left(\pi^{0} / \eta \rightarrow \gamma \gamma\right.$ decays, photons from initial-state radiation, and other). Efficiency for each criterion is given in the order it is applied. 


\begin{tabular}{|c|c|c|c|c|c|}
\hline \multirow[b]{2}{*}{ Sec. } & \multirow[b]{2}{*}{ Description } & \multicolumn{2}{|c|}{$B^{0} B^{0}$} & \multicolumn{2}{|c|}{$B^{+} B^{-}$} \\
\hline & & $\pi^{0} / \eta$ & other & $\pi^{0} / \eta$ & other \\
\hline 3.2 & Photon with $1.5<E_{\gamma}^{*}<3.5 \mathrm{GeV}$ & \multicolumn{2}{|c|}{0.024} & \multicolumn{2}{|c|}{0.023} \\
\hline 3.2 & QED continuum rejection & \multicolumn{2}{|c|}{0.972} & \multicolumn{2}{|c|}{0.971} \\
\hline & Cumulative Preselection & \multicolumn{2}{|c|}{0.0234} & \multicolumn{2}{|c|}{0.0231} \\
\hline 3.3 .1 & $-0.74<\cos \theta_{\gamma}<0.93$ & 0.97 & 0.96 & 0.97 & 0.95 \\
\hline 3.3 .1 & No problematic channels & 0.98 & 0.98 & 0.98 & 0.98 \\
\hline 3.3 .1 & $\mathrm{n}_{\text {Crystals }} \geq 4$ & 1.00 & 0.99 & 1.00 & 0.99 \\
\hline 3.3 .2 & Second Moment $<0.002 \mathrm{rad}^{2}$ & 0.96 & 0.40 & 0.96 & 0.44 \\
\hline 3.3 .2 & $L A T<0.45$ & 1.00 & 0.91 & 1.00 & 0.93 \\
\hline 3.3 .2 & Bump Isolation $>25 \mathrm{~cm}$ & 0.65 & 0.70 & 0.64 & 0.73 \\
\hline 3.3 .3 & $\pi^{0}$ veto & 0.42 & 0.83 & 0.40 & 0.84 \\
\hline \multirow[t]{2}{*}{3.3 .3} & $\eta$ veto & 0.69 & 0.82 & 0.71 & 0.82 \\
\hline & Cumulative Photon Selection & 0.175 & 0.159 & 0.169 & 0.189 \\
\hline 3.4 & $20 \mathrm{GeV}<E_{\gamma}^{*}<2.7 \mathrm{GeV}$ & 0.112 & 0.130 & 0.108 & $\overline{0.114}$ \\
\hline 3.5 & $\bar{R}_{2}^{*}<0.55$ & 0.98 & 1.00 & 0.98 & 1.00 \\
\hline \multirow[t]{2}{*}{3.5} & Fisher Discriminant $>0.575$ & 0.46 & 0.26 & 0.47 & 0.27 \\
\hline & Cumulative Event Shape & 0.45 & 0.26 & 0.46 & 0.27 \\
\hline \multirow[t]{3}{*}{3.6} & "Single Photon Candidate & 1.00 & 0.99 & 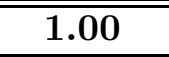 & 0.99 \\
\hline & \multirow{2}{*}{ Expected Events, $80.5 \mathrm{fb}^{-1}$} & 6468 & 1658 & 6594 & 1328 \\
\hline & & \multicolumn{2}{|c|}{8127} & \multicolumn{2}{|c|}{7921} \\
\hline 3.7 & Electron Tag $p_{e}^{*}>1.25 \mathrm{GeV} / c$ & 0.048 & 0.036 & 0.056 & 0.048 \\
\hline 3.7 & $\cos \theta_{\gamma e}>-0.7$ & 0.64 & 0.69 & 0.59 & 0.63 \\
\hline 3.7 & Muon Tag $p_{\mu}^{*}>1.5 \mathrm{GeV} / c$ & 0.025 & 0.014 & 0.026 & 0.019 \\
\hline 3.7 & $\cos \theta_{\gamma \mu}>-0.7$ & 0.54 & 0.62 & 0.49 & 0.63 \\
\hline \multirow[t]{2}{*}{3.7} & $E_{\text {miss }}^{*}>0.8 \mathrm{GeV}$ & 0.98 & 0.77 & 0.98 & 0.79 \\
\hline & Cumulative Lepton Tag & 0.043 & 0.026 & 0.044 & 0.034 \\
\hline \multirow[t]{5}{*}{ 13.9 } & $N_{\text {mult }} \geq 4.5$ & 0.998 & 1.000 & \begin{tabular}{c|}
0.994 \\
\end{tabular} & 1.000 \\
\hline & \multirow{2}{*}{ TOTAL Efficiency } & $9.07 \mathrm{e}-06$ & $3.25 \mathrm{e}-06$ & $8.47 \mathrm{e}-06$ & $4.48 \mathrm{e}-06$ \\
\hline & & \multicolumn{2}{|c|}{$7.32 \mathrm{e}-06$} & \multicolumn{2}{|c|}{$7.57 \mathrm{e}-06$} \\
\hline & \multirow{2}{*}{ Expected Events, $80.5 \mathrm{fb}^{-1}$} & 280 & 43 & 290 & 45 \\
\hline & & \multicolumn{2}{|c|}{323} & \multicolumn{2}{|c|}{335} \\
\hline
\end{tabular}

Table 3.5: Selection efficiencies and expected number of events in $80.5 \mathrm{fb}^{-1}$ for $B \bar{B}$ background, determined from Monte Carlo samples. The background is divided into its principal components $\left(\pi^{0} / \eta \rightarrow \gamma \gamma\right.$ decays and other; the latter includes backgrounds from hadrons interacting in the EMC). 
reduces the frequency of the background showers from charged particles by keeping the location of the deposit well within the tracking coverage.

- Crystal quality. The candidate shower should have hits from at least four crystals (the partial weights from crystals shared with other showers are added) [54]. None of the crystals in the cluster should be dead or noisy, nor be served by a faulty read-out module or noisy power supply.

\subsubsection{Shower Shape and Isolation Requirements}

The fine segmentation of the calorimeter provides means of separating electromagnetic showers from hadronic showers. Electromagnetic showers deposit most of their energy in a few crystals, with smaller lateral spread. Hadronic showers tend to have wider and more irregular shower profiles. To quantify and exploit these differences, various variables have been defined in the literature. They range in complexity from relatively straight-forward variables such as the ratio of the energy deposited in a few central crystals to the total energy, to more advanced ones in which the energy deposited in each crystal is assigned a weight depending on its location. The choice of the variables is a compromise between better rejection power and the systematic biases that might arise from the imperfect modelling of the interactions in the calorimeter.

The determination of the most useful technique should take the specific nature of the $B \rightarrow X_{s} \gamma$ photon and the backgrounds into account. The optimization for the best background rejection without any significant loss in signal photon has been carried out simultaneously with the studies of systematic uncertainties. Monte Carlo simulated photons are compared to pure samples of photons from virtual Compton scattering events. The best performance is achieved for a combination of two criteria:

- Two-dimensional second moment, $\sum_{i} f_{i} \cdot\left[\left(\Delta \theta_{i}\right)^{2}+\left(\Delta \phi_{i}\right)^{2}\right]$, is required to be less than $0.002 \mathrm{rad}^{2}$. Here $f_{i}$ is the fraction of a candidate's total energy contributed by crystal $i$ and $\left(\Delta \theta_{i}, \Delta \phi_{i}\right)$ is the position of that crystal relative to the candidate's centroid (Section 2.4). 
- The lateral moment ( $L A T)$ should be less than 0.45. This parameter, first introduced at the Argus experiment, is similar to the second moment, but treats the two most-energetic crystals distinctly, to exploit the fact that electromagnetic showers deposit largest portion of their energy in 2-3 crystals [55]:

$$
L A T=\frac{\sum_{i=3}^{n} E_{i} \cdot r_{i}^{2}}{\left(E_{1}+E_{2}\right) \cdot r_{0}^{2}+\sum_{i=3}^{n} E_{i} \cdot r_{i}^{2}}
$$

Here, $E_{i}$ is the energy measured in $i^{\text {th }}$ crystal, ordered such that $E_{i}>E_{i+1} \cdot r_{i}$ is the distance from the $i^{\text {th }}$ crystal to the center of the shower (not its centroid), which is defined as the ordinary center-of-gravity. Note that the two crystals with the highest energies are given extra weight, $r_{0}^{2}$, by setting $r_{0}=5 \mathrm{~cm}$, the average width of a crystal. Although highly correlated with the second moment, $L A T$ still provides considerable extra rejection as illustrated in Figure 3.1.
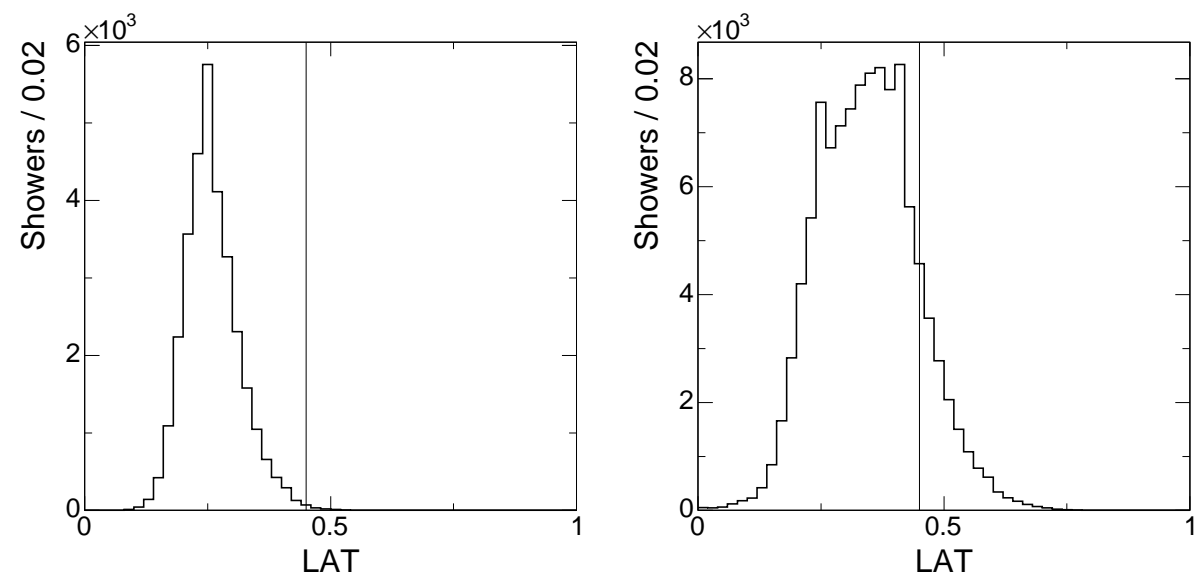

Figure 3.1: MC simulation of the lateral moments of the high-energy photon candidates, after the requirement on the second moment and the photon quality criteria have been applied. Photon candidates from signal events (left) and hadronic showers from the $B \bar{B}$ background (right) are shown. The vertical lines indicate the maximum value of the lateral moment accepted.

These criteria are effective in reducing certain backgrounds from $\pi^{0}$ decays as well. In the decays of $\pi^{0}$ mesons with energies above $3 \mathrm{GeV}$, the two photons may result in a single cluster that cannot be separated into two showers. Most such clusters, referred to as merged $\pi^{0} s$, have elongated shower profiles and are rejected by the shower shape requirements. 
One final requirement on the showers is that they be separated from any other shower in the EMC by at least $25 \mathrm{~cm}$. This isolation requirement is helpful against all types of backgrounds except the photons from continuum ISR (Figure 3.2).
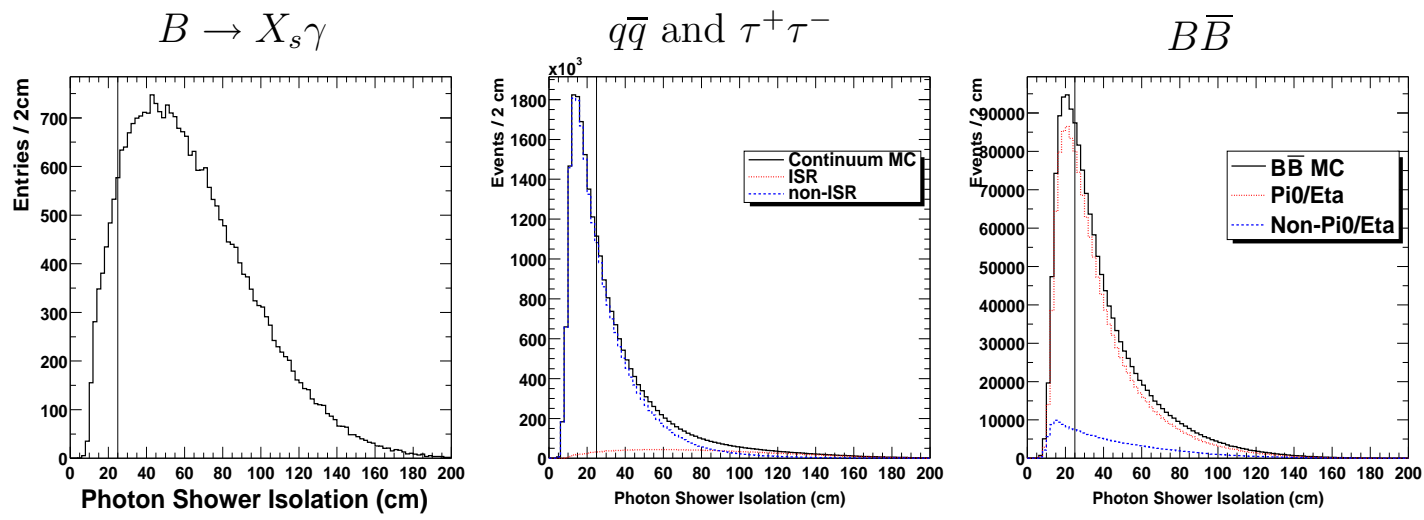

Figure 3.2: Distribution of the distance $(\mathrm{cm})$ at the EMC between a highenergy photon candidate and the nearest other shower, from MC simulation. The vertical lines indicate the minimum distance required for a candidate.

\subsection{3 $\pi^{0}$ and $\eta$ Vetoes}

The decays of $\pi^{0} \rightarrow \gamma \gamma$ and $\eta \rightarrow \gamma \gamma$ are a principle source of background photons. This background can be reduced by eliminating any photon candidate that is part of a visible photon pair with mass close to the mass of $\pi^{0}$ or $\eta$ mesons. Any high-energy photon candidates are vetoed if, when combined with any other photon in the event fulfilling a minimum energy requirement, they fall within the $\pi^{0}(\eta)$ mass window $115(508)<M_{\gamma \gamma}<155(588) \mathrm{MeV}$. Figure 3.3 shows the invariant mass distributions for all possible combinations of such photons with the high-energy photon.

Most of the background stems from this source, and the most problematic part of it is found in non-radiative $B \bar{B}$ events which dominate at lower photon energies but are less significant in the higher range of the signal spectrum. To best reduce this particular background, for $E_{\gamma}^{*}<2.3 \mathrm{GeV}$, more stringent requirements are applied on the minimum laboratory energy of the second $\pi^{0}(\eta)$ daughter photon, as tabulated in 

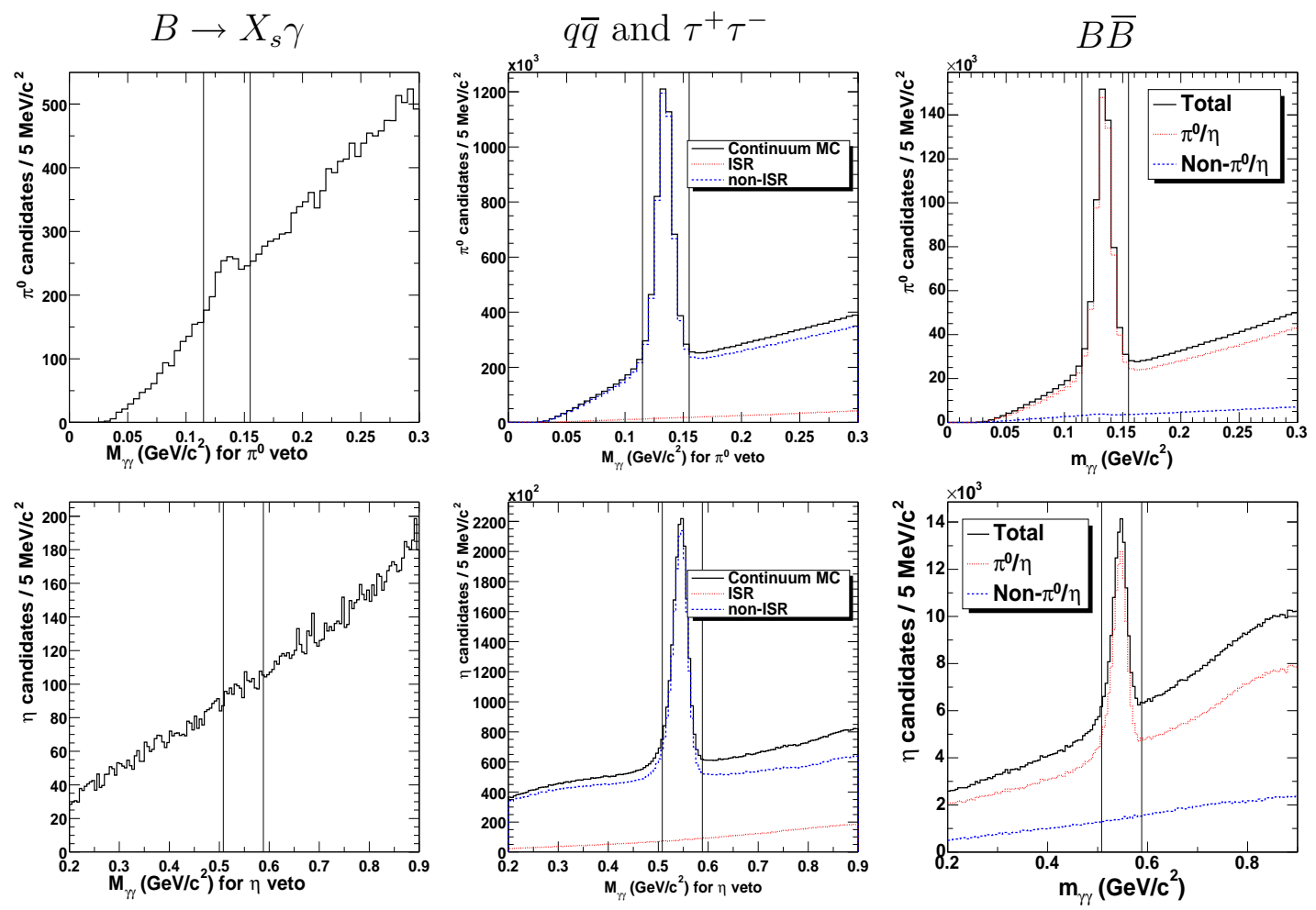

Figure 3.3: The $M_{\gamma \gamma}$ distribution for all combinations of the high-energy photon with all other photons meeting the minimum energy requirement, from MC simulation in the $\pi^{0}$ (top) and $\eta$ (bottom) regions. The vertical lines indicate the mass windows employed in the vetoes.

Table 3.6. This method improves the expected statistical significance of the branching fraction measurement by about $9 \%$ over an $E_{\gamma}^{*}$-independent treatment.

\subsection{Selection of the Photon Energy Range}

One extra requirement is applied to the photon candidates, but it is best treated independently of the others. Figure 3.4 shows the $E_{\gamma}^{*}$ distribution for all high-energy photon candidates after the photon selection cuts of Section 3.3. With rapidly rising backgrounds from $B \bar{B}$ events below $2.2 \mathrm{GeV}$ and only continuum backgrounds above 


\begin{tabular}{|l|c|c|}
\hline & $<2.3 \mathrm{GeV}$ & $\geq 2.3 \mathrm{GeV}$ \\
\hline \hline$\pi^{0}$ veto & $E_{\gamma 2} \geq 40 \mathrm{MeV}$ & $E_{\gamma 2} \geq 80 \mathrm{MeV}$ \\
\hline$\eta$ veto & $E_{\gamma 2} \geq 175 \mathrm{MeV}$ & $E_{\gamma 2} \geq 275 \mathrm{MeV}$ \\
\hline
\end{tabular}

Table 3.6: The minimum $E_{\gamma 2}$ requirements for reconstructing a $\pi^{0}$ or $\eta$ candidate with the high-energy photon in the different regions of $E_{\gamma}^{*}$.

$2.8 \mathrm{GeV}$ (approximate kinematic limit of the signal distribution), at this stage it becomes useful to restrict the analysis to a signal-enriched part of the energy spectrum. This choice relates mainly to the optimization process of Section 3.8, and is revisited after the full assessment of the expected systematic errors (Section 5.3).

For the lower boundary of the region, $E_{\gamma}^{*}>2.0 \mathrm{GeV}$ is chosen. This choice is motivated by the size of the systematic uncertainties from $B \bar{B}$ background substraction that was observed in previous $B \rightarrow X_{s} \gamma$ measurements (particularly [56]). For the upper boundary, the requirement is $E_{\gamma}^{*}<2.7 \mathrm{GeV}$, because of the very small amount of signal expected this close to the kinematic limit. The effect of the upper boundary by itself on the signal efficiency at this stage of the event selection is about $93 \%$ for the $B \rightarrow K^{*} \gamma$ modes, but $99.7 \%$ for the KN480 samples.
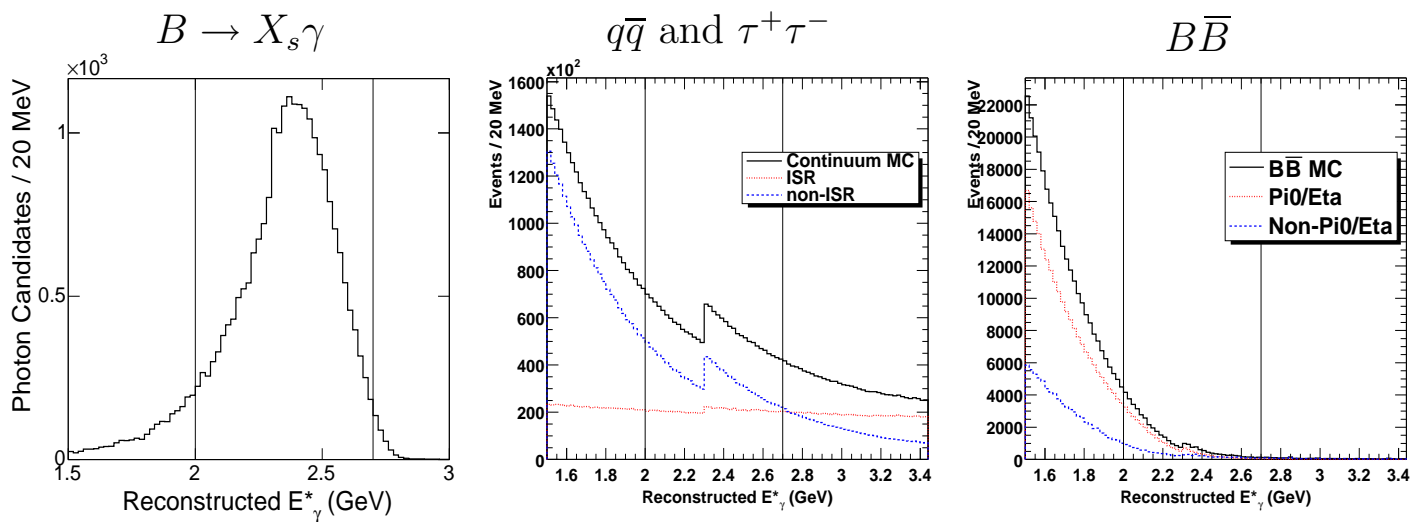

Figure 3.4: The $E_{\gamma}^{*}$ distribution of the high-energy photon, from MC simulation, after the photon selection criteria are applied. The vertical lines indicate the range chosen at this stage of the event selection (Section 3.4). This range is revised later (Section [5.3). 


\subsection{Event Topology}

In the c.m. frame, the event topology for $B \bar{B}$ decays differ from the continuum decays. Since the $B$ mesons are almost at rest in this frame, signal and $B \bar{B}$ background events are isotropic, whereas the particles in continuum backgrounds tend to form two roughly collinear jets recoiling against each other. The only exception to this is the ISR component of the continuum backgrounds, where the photon recoils against the rest frame of the jets (or the rest frame of the high-momentum tracks for background processes like $\left.e^{+} e^{-} \gamma, \mu^{+} \mu^{-} \gamma\right)$.

To distinguish these different topologies, a set of variables are used. These variables are computed using all the good tracks and the good photons as defined in Section 3.1. With the exception of the $R_{2}^{\prime} / R_{2}^{*}$, they are all calculated in the c.m. frame.

$R_{2}^{*} \quad$ The normalized second Fox-Wolfram moment is defined as the ratio of the second and zeroth Fox-Wolfram moments, $H_{l}=\sum_{i, j} \frac{\left|P_{i}\right|\left|P_{j}\right|}{E_{\text {total }}^{2}} \cdot P_{l}\left(\cos \theta_{i j}\right)$, where $P_{i, j}$ and $\theta_{i j}$ are the momenta of and the opening angle between particles $i$ and $j$, $E_{\text {total }}=\sum_{i} E_{i}$ is the total visible energy and $P_{l}(x)$ are the Legendre polynomials [57]. $R_{2}^{*}$ takes a value between 0 and 1 , with lower values indicating more spherical events.

$E_{f 1}^{*}-E_{f 9}^{*} \quad$ Nine $10^{\circ}$ cones are defined around the candidate photon in the c.m. frame, from 0 to $90^{\circ}$. $E_{f i}^{*}$ is the energy flow through the $i^{\text {th }}$ cone, i.e. the energy sum of all particles, whose momentum vectors lie within $10(i-1)$ to $10 i$ degrees with respect to the direction of the photon. The high-energy photon itself is excluded from the sum.

$E_{b 1}^{*}-E_{b 9}^{*} \quad$ The same as for $E_{f}^{*}$ except that the cones are 180 to $90^{\circ}$ from the direction of the candidate photon, i.e. $E_{b 1}^{*}$ covers the range $170-180^{\circ}$.

$R_{2}^{\prime} / R_{2}^{*} \quad$ The ratio of $R_{2}^{\prime}$ and $R_{2}^{*}$. Here $R_{2}^{\prime}$ is the normalized second Fox-Wolfram moment computed in the recoil frame, in which the momenta of the beams and the candidate photon satisfy the relation $\vec{p}\left(e^{+}\right)+\vec{p}\left(e^{-}\right)-\vec{p}(\gamma)=0$. In this frame, the two-jet system of an ISR event would be at rest. Unlike $R_{2}^{*}$, the momentum and the energy of the photon candidate are excluded from the calculation of $R_{2}^{\prime}$. 
Most of these variables do not have a particularly strong discrimination power, but all have some. It is possible to choose a few of them and apply selection criteria one-by-one. However, because of the correlations, such a method is not as efficient as combining them (or subsets of them) through a multivariate technique. (A improvement of $18 \%$ is observed in the figure of merit of Section 3.8 compared to [56.)

The particular multivariate technique chosen in this analysis is the Fisher discriminant [58]. In this technique, a vector of input variables $\boldsymbol{x}$ is projected on to an output variable, $y=F(\boldsymbol{x})$, such that for two classes of events (signal and continuum background), the ratio of the between-class variance of $y$ to its within-class variance is maximized. The transformation is a linear function $F(\boldsymbol{x})=\boldsymbol{c} \cdot \boldsymbol{x}+d$, where the Fisher coefficients are given by $\boldsymbol{c}=N\left(\overline{\boldsymbol{x}}_{\boldsymbol{s i g}}-\overline{\boldsymbol{x}}_{\boldsymbol{b k g} \boldsymbol{g}}\right)^{\mathrm{T}} \cdot \boldsymbol{W}^{-1}$, with $N$ a normalization factor, $\boldsymbol{W}$ the within-class covariance matrix and $\overline{\boldsymbol{x}}_{\boldsymbol{s i g} \boldsymbol{g} \boldsymbol{k \boldsymbol { k }}}$ the mean value vectors for input variables in signal and background classes.

This technique, being a linear discriminant method, is selected over non-linear alternatives like neural nets, particularly because of its relative transparency and the corresponding ease in implementation and systematic studies. The BABAR package used for the computation of the coefficients [59] also returns a value for the arbitrary offset $d$, so that the likelihood for an event to be signal or background will be equal at $y=0$.

Various different sets of input variables have been studied. The best performance has been observed for a discriminant incorporating the photon-candidate-dependent variables listed above $\left(R_{2}^{\prime} / R_{2}^{*}\right.$ and the flow cone variables $)$, combined with a separate selection criterion on $R_{2}^{*}$. Since the shape variables primarily discriminate between signal and continuum background, only samples for those two categories have been employed. Samples of 3354 signal (about 5:1 mix of $X_{s} \gamma$ and $K^{*} \gamma$ ) and continuum (a properly weighted mix of $q \bar{q}$ and $\tau^{+} \tau^{-}$) Monte Carlo events which already satisfy the photon selection and $E_{\gamma}^{*}$ criteria and the lepton tag requirement (except for the criterion on missing energy), and which contain only one high-energy photon candidate, are used. (The tag requirements of Section 3.7 are applied, since they have significant effects on event topology.) The computed Fisher parameters are listed in Table 3.7.

With these coefficients (and the offset value), a Fisher discriminant output value 


\begin{tabular}{|c|c|c|}
\hline Input variable & Coefficient & Coeff $* \Delta($ mean $)$ \\
\hline 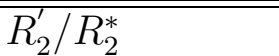 & -0.8627 & 0.731 \\
\hline$E_{f 1}^{*}\left(0-10^{\circ}\right)$ & $-0.8715 \mathrm{GeV}^{-1}$ & 0.097 \\
\hline$E_{f 2}^{*}\left(10-20^{\circ}\right)$ & $-0.7637 \mathrm{GeV}^{-1}$ & 0.094 \\
\hline$E_{f 3}^{*}\left(20-30^{\circ}\right)$ & $-0.4282 \mathrm{GeV}^{-1}$ & 0.017 \\
\hline$E_{f 4}^{*}\left(30-40^{\circ}\right)$ & $-0.2881 \mathrm{GeV}^{-1}$ & -0.013 \\
\hline$E_{f 5}^{*}\left(40-50^{\circ}\right)$ & $-0.1552 \mathrm{GeV}^{-1}$ & -0.012 \\
\hline$E_{f 6}^{*}\left(50-60^{\circ}\right)$ & $-0.1226 \mathrm{GeV}^{-1}$ & -0.014 \\
\hline$E_{f 7}^{*}\left(60-70^{\circ}\right)$ & $-0.0567 \mathrm{GeV}^{-1}$ & -0.005 \\
\hline$E_{f 8}^{*}\left(70-80^{\circ}\right)$ & $-0.0016 \mathrm{GeV}^{-1}$ & -0.000 \\
\hline$E_{f 9}^{*}\left(80-90^{\circ}\right)$ & $0.0343 \mathrm{GeV}^{-1}$ & 0.001 \\
\hline$E_{b 1}^{*}\left(170-180^{\circ}\right)$ & $0.1380 \mathrm{GeV}^{-1}$ & 0.035 \\
\hline$E_{b 2}^{*}\left(160-170^{\circ}\right)$ & $0.0756 \mathrm{GeV}^{-1}$ & 0.030 \\
\hline$E_{b 3}^{*}\left(150-160^{\circ}\right)$ & $-0.0664 \mathrm{GeV}^{-1}$ & -0.010 \\
\hline$E_{b 4}^{*}\left(140-150^{\circ}\right)$ & $-0.1387 \mathrm{GeV}^{-1}$ & 0.003 \\
\hline$E_{b 5}^{*}\left(130-140^{\circ}\right)$ & $-0.2529 \mathrm{GeV}^{-1}$ & 0.058 \\
\hline$E_{b 6}^{*}\left(120-130^{\circ}\right)$ & $-0.1587 \mathrm{GeV}^{-1}$ & 0.040 \\
\hline$E_{b 7}^{*}\left(110-120^{\circ}\right)$ & $-0.0512 \mathrm{GeV}^{-1}$ & 0.010 \\
\hline$E_{b 8}^{*}\left(100-110^{\circ}\right)$ & $0.0319 \mathrm{GeV}^{-1}$ & -0.005 \\
\hline$E_{b 9}^{*}\left(90-100^{\circ}\right)$ & $0.0344 \mathrm{GeV}^{-1}$ & -0.003 \\
\hline "Offset" $(d)$ & & \\
\hline
\end{tabular}

Table 3.7: The coefficients of the Fisher discriminant and the additive offset. For each input variable, $x_{i}$, its corresponding coefficient, $c_{i}$, and a simple measure of its separating power (the product of the coefficient with the difference between the means of the variable in signal and background samples, $\left.c_{i} \cdot\left(\bar{x}_{i, s i g}-\bar{x}_{i, b k g}\right)\right)$ are given. This measure cannot account for the correlations or the shapes of the distributions. 

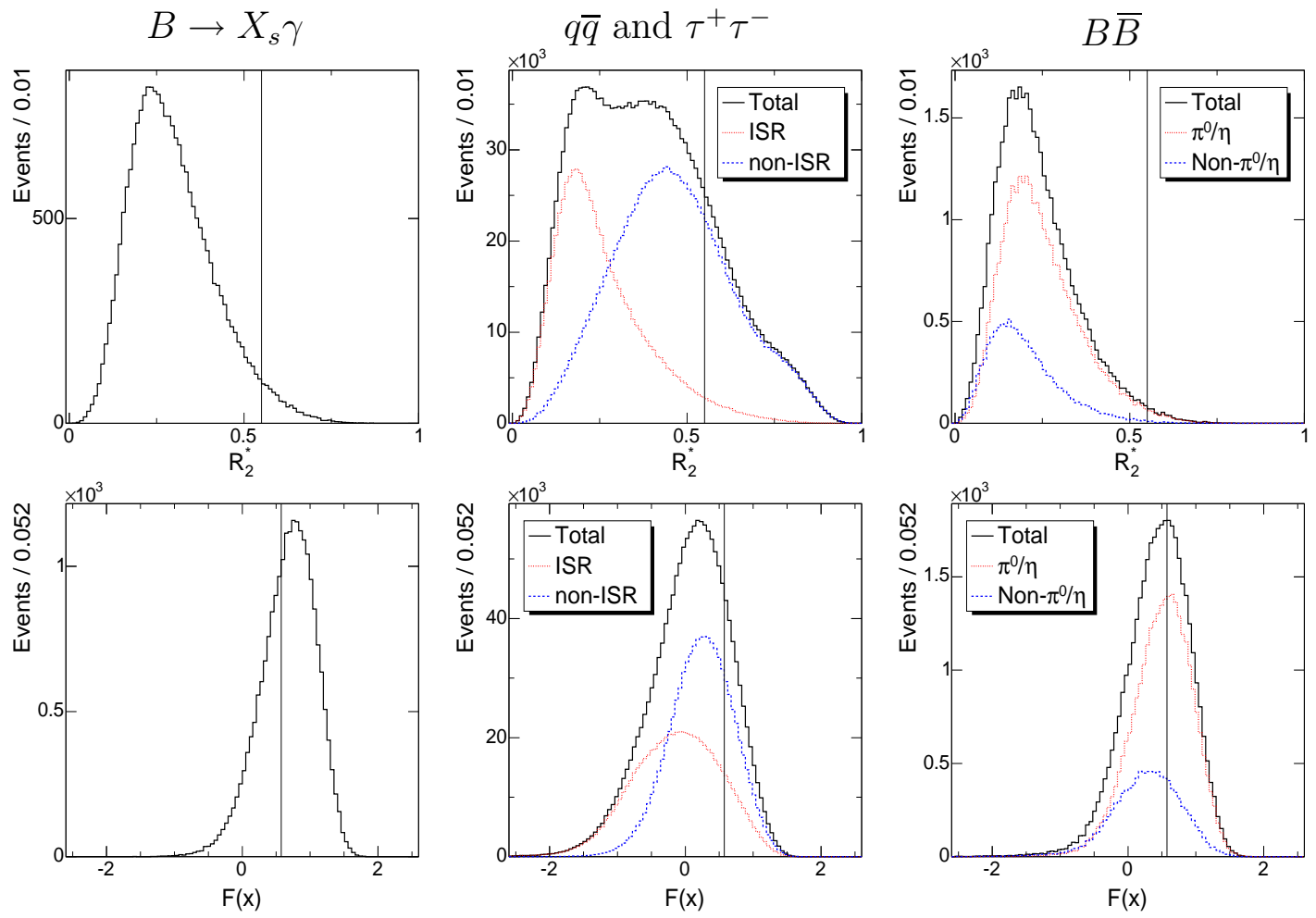

Figure 3.5: The $R_{2}^{*}$ (top) and Fisher discriminant distributions from MC simulation of signal and background events, after previous selection criteria (that includes the requirement on the $R_{2}^{*}$ for the Fisher discriminant plot). The vertical lines indicate the edge of the accepted region.

is calculated for each event. The full optimization (Section 3.8) indicates the best choice for the restrictions to be applied on this output and normalized Fox-Wolfram moment: $R_{2}^{*}<0.55$ and $F(\boldsymbol{x})>0.575$. The distributions of both quantities, after the earlier requirements have already been applied, are shown in Figure 3.5. Two small features of these distributions deserve to be pointed out here: First, the continuumbackground $R_{2}^{*}$ distribution does not have a sharp cutoff at 0.9 , although the plotted events satisfy an earlier preselection requirement on $R_{2}^{*}$ (Section 3.2). This is a result of the addition of the neutral particles (particularly the high-energy photon candidate) into the computation of $R_{2}^{*}$. Second, although the offset $(d)$ mentioned above has been used, both the signal and background Fisher discriminant distributions have positive 
mean values. If the signal and continuum distributions are normalized to the same area and overlayed, they intersect at around 0.4, not at zero, which would be expected because of the offset. The reason is for this apparent disagreement is the simple fact that Figure 3.5 shows events before the lepton tagging requirements, and it is largely the requirement on the angle between the photon and the high-momentum lepton that significantly changes the average event topology and shifts the distribution to lower values.

\subsection{Removal of Multiple-Candidate Events}

As mentioned in Section 3.3, 2.5\% of the signal Monte Carlo events have multiple photon candidates after the preselection. Photon selection criteria reduce this fraction by a factor of five, and the restriction of the energy range by almost an order of magnitude. With the requirements on the event topology, very few (less than $0.05 \%$ ) events remain with multiple photon candidates. Those events are simply discarded.

As an additional exercise, the minimum $E_{\gamma}^{*}$ requirement of $2.0 \mathrm{GeV}$ (Section 3.4) can be taken out the analysis chain. This manner of assessing the multi-candidate fraction is particularly important for the extraction of the full energy spectrum. Fortunately, when this exercise is done, the obtained value is $0.15 \%$, essentially negligible for the spectrum analysis.

\subsection{Lepton Tagging}

Approximately $20 \%$ of $B$-meson decays are semileptonic with a prompt electron or muon in the final state [4. A significant portion of these leptons can have high momenta, $p^{*} \gtrsim 1 \mathrm{GeV}$. Leptons of high momentum do appear also in the final states of $q \bar{q}$ events, but with a much lower frequency. Therefore, in a given sample of multihadronic events collected at the $\Upsilon(4 S)$ resonance, it is possible to greatly enhance the likelihood of finding a $B \bar{B}$ event, by restricting the sample to tagged events, i.e. a subsample of events with at least one high-momentum lepton.

Such a restriction is obviously very costly in terms of available statistics. It can be 
shown that to be meaningful, the tagging efficiency in signal events should be higher than the squareroot of the tagging efficiency in continuum events. This requires not only a careful optimization of the minimum accepted lepton momentum, but also further kinematic constraints on the tag [60]. But before going into the details of those constraints, it is useful to discuss the lepton identification algorithms. The identification should have high efficiency and purity.

\subsubsection{Lepton Identification}

All lepton candidates are selected from the good tracks (Section [3.1), but with tighter requirements on the distance of closest approach to the interaction point: $d_{0}^{I P}<0.4 \mathrm{~cm}$ and $\left|z_{0}^{I P}\right|<4 \mathrm{~cm}$. Unlike some other charged particles that might originate away from the IP (for example the pions from the decays of the $K_{S}^{0}$ mesons), the leptons of interest for tagging are those from the prompt decays of the $B$ mesons.

The following criteria are applied for the identification of the electrons [61:

- $\mathrm{d} E / \mathrm{d} x$ as measured by the tracking system is required to be within $-2.2 \sigma$ to $+7.0 \sigma$ of its mean value for electrons.

- The Cherenkov angle measured by the DIRC should be within $3 \sigma$ of the electron hypothesis. This requirement is applied only if there are at least 10 Cherenkov photons associated with the track.

- An EMC shower of at least 3 crystals should be matched to the track.

- The ratio of the energy of the associated shower to the momentum of the track should be in the range $0.89<E / p<1.2$.

- The lateral profile for the EMC shower should be consistent with the profile for electromagnetic showers. For the particular purpose of electron identification, the following shower shape criteria have been found most useful:

The lateral moment (Section 3.3.2) should be less than 0.6.

The Zernike moment $(4,2)$ should be in the range $-10<A_{42}<0.11$. These moments, first introduced at the ZEUS experiment [62, are particularly sensitive 
to the irregularities in the shapes of the hadronic showers. They are defined as: $A_{n m}=\sum_{r_{i} \leq R_{0}}^{n} f_{i} \cdot F_{n m}^{Z}\left(\frac{r_{i}}{R_{0}}\right) \cdot e^{-i m \phi_{i}}$, where $R_{0}=15 \mathrm{~cm}, f_{i}$ is the fraction of the shower's total energy contributed by crystal $i$, and $F_{n m}^{Z}(x)$ are the Zernike functions, with $f_{42}(x)=4 x^{4}-2 x^{2}$. For the computation of the Zernike moments, like the lateral moment, the position of a crystal is not measured with respect to the shower centroid, but relative to the ordinary center-of-gravity with linear weights.

- In addition to the requirements on the lateral shower shape, it is possible to exploit the longitudinal properties of the showers associated with charged tracks. Electrons shower shortly after entering the calorimeter, while hadronic interactions tend to reach their maximum deeper inside the crystals. Therefore the azimuthal distance (as measured from the IP) between the shower's position, $\phi_{S}$, and the track's entrance point to the EMC, $\phi_{T}^{E M C}$, is usually longer for hadrons. An requirement dependent on the particle's charge and transverse momentum is made on this distance: $-0.02<q \cdot\left(\phi_{T}^{E M C}-\phi_{S}\right)<\left(0.10 e^{-1.9 p_{T}}+0.03\right) \mathrm{rad}$.

The muon candidates are subjected to the following criteria 63]:

- The energy deposited in the EMC should be consistent with a MIP, i.e. in the range $50-400 \mathrm{MeV}$.

- The number of IFR layers with hits associated with the track should be $N_{L} \geq 2$.

- The track is required to have transversed at least 2.2 nuclear interaction lengths in the detector, $N_{\lambda}>2.2$.

- The difference between the $N_{\lambda}$ and the expected number of interaction lengths that would be transversed by a muon of the measured momentum should not be more than 1.0 .

- The average number of hits per IFR layer and the RMS of the distribution should be less than 8.0 and 4.0, respectively. 
- The $\chi^{2}$ per degree of freedom of a poynomial fit to the clusters of IFR hits should be less than 3.0. Additionally, the $\chi^{2}$ per degree of freedom of a match between the extrapolated track from the inner detectors and those IFR clusters should be less than 5.0.

- For tracks that transverse at least a portion of the IFR forward endcap, a continuity criterion is applied in order to reduce the pion misidentification rate due to artificially high $N_{\lambda}$ caused by machine backgrounds: At least $30 \%$ of the layers between the innermost and outermost hit layers should record hits.

- Finally, a tight kaon veto is applied on the muon candidates, based on the track's measured $\mathrm{d} E / \mathrm{d} x$ and Cherenkov information [64].

The efficiencies and the misidentification probabilities (from pions) of these criteria were presented earlier (Figures 2.8 and 2.11). Electrons can be selected with high efficiency and purity for momenta above approximately $0.75 \mathrm{GeV} / c$. Muons can be identified with reasonable efficiency (60\%) above momenta of approximately $1.0 \mathrm{GeV} / c$. However the pion misidentification rate is an order of magnitude higher for muons than electrons. The kaon misidentification rate is negligible in both cases above $1.0 \mathrm{GeV} / c$.

Unfortunately, the predictions of the simulation are not satisfactory enough to give particle identification (PID) efficiencies and misidentification rates that are consistent with the data. Therefore, these rates are corrected by substituting the measured values based on data control samples into the simulation. The effects of this correction are readily included in the selection efficiency tables (Tables 3.2 3.5) and all the plots and discussions in this and following chapters. Section 6.3.1 describes the correction and the relevant systematic errors associated with it.

\subsubsection{Kinematic Requirements on Lepton Candidates}

Figure 3.6 shows the simulated c.m. momentum distributions for the highest-momentum electrons and muons (if any) satisfying the requirements noted above. 

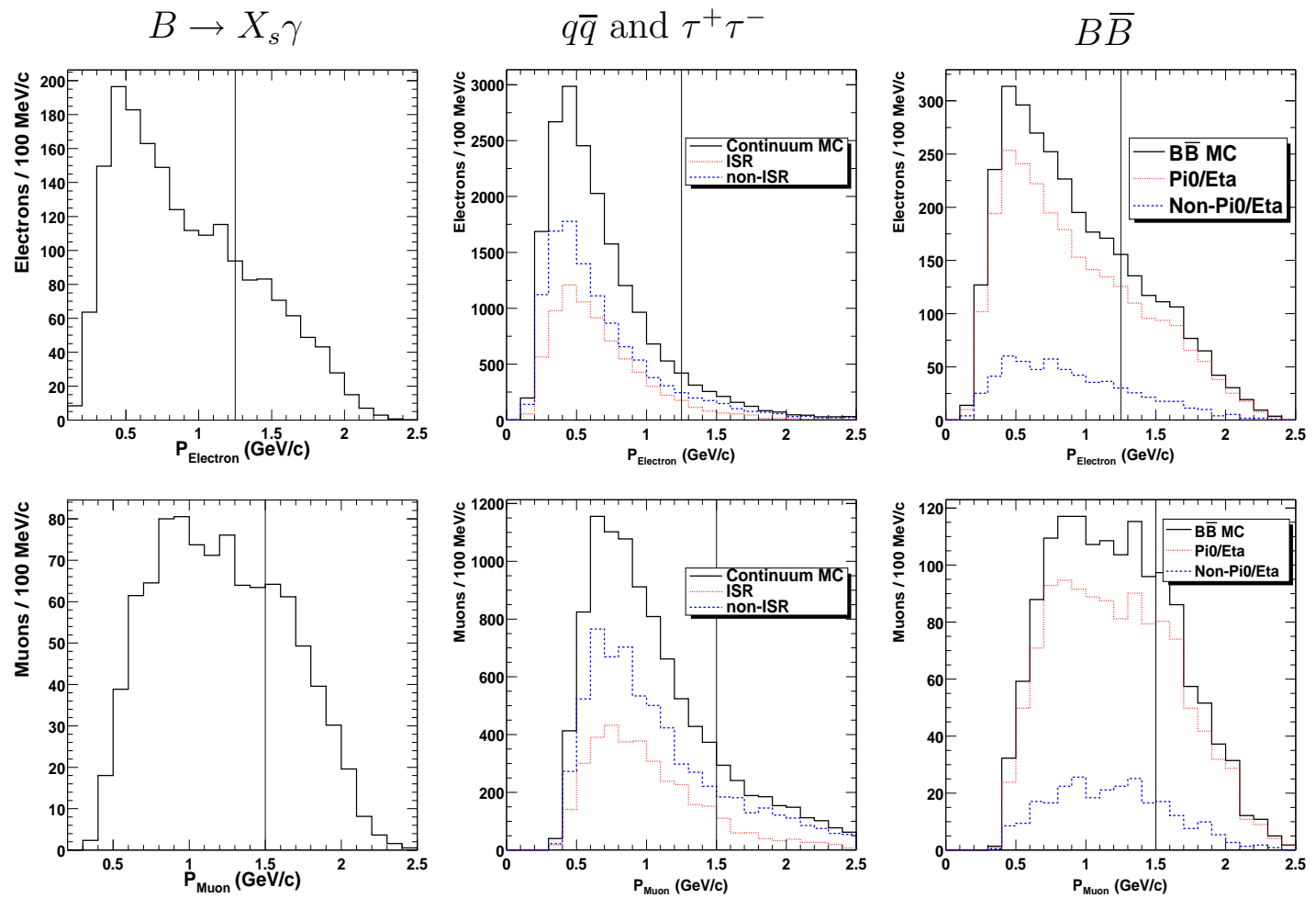

Figure 3.6: The c.m.-frame momentum distributions from MC electron (top) and muon (bottom) candidates. When multiple candidates are present in an event, the one with the highest momentum is plotted. The vertical lines indicate the minimum momentum required.

To best exploit the differences between the simulated continuum and $B \bar{B}$ lepton spectra, the following restrictions on the minimum momenta are found to be most useful: $p_{e}^{*}>1.25 \mathrm{GeV}$ and $p_{\mu}^{*}>1.5 \mathrm{GeV}$. If both an electron and a muon candidate are present in an event satisfying their respective requirements, the electron is given preference. The comparison of the spectra might suggest an upper bound on the momenta as well, particularly for muons around $2.2 \mathrm{GeV} / c$, but after the angle and missing-energy requirements below, no events survive in that region.

The tagging lepton candidate can be used to construct a new shape variable, by considering the angle between its momentum and that of the high-energy photon in c.m. frame. Figure 3.7 shows that the cosine of this angle gives significant additional 
discrimination. $\cos \theta_{\gamma e}\left(\cos \theta_{\gamma \mu}\right)>-0.7$ is required.
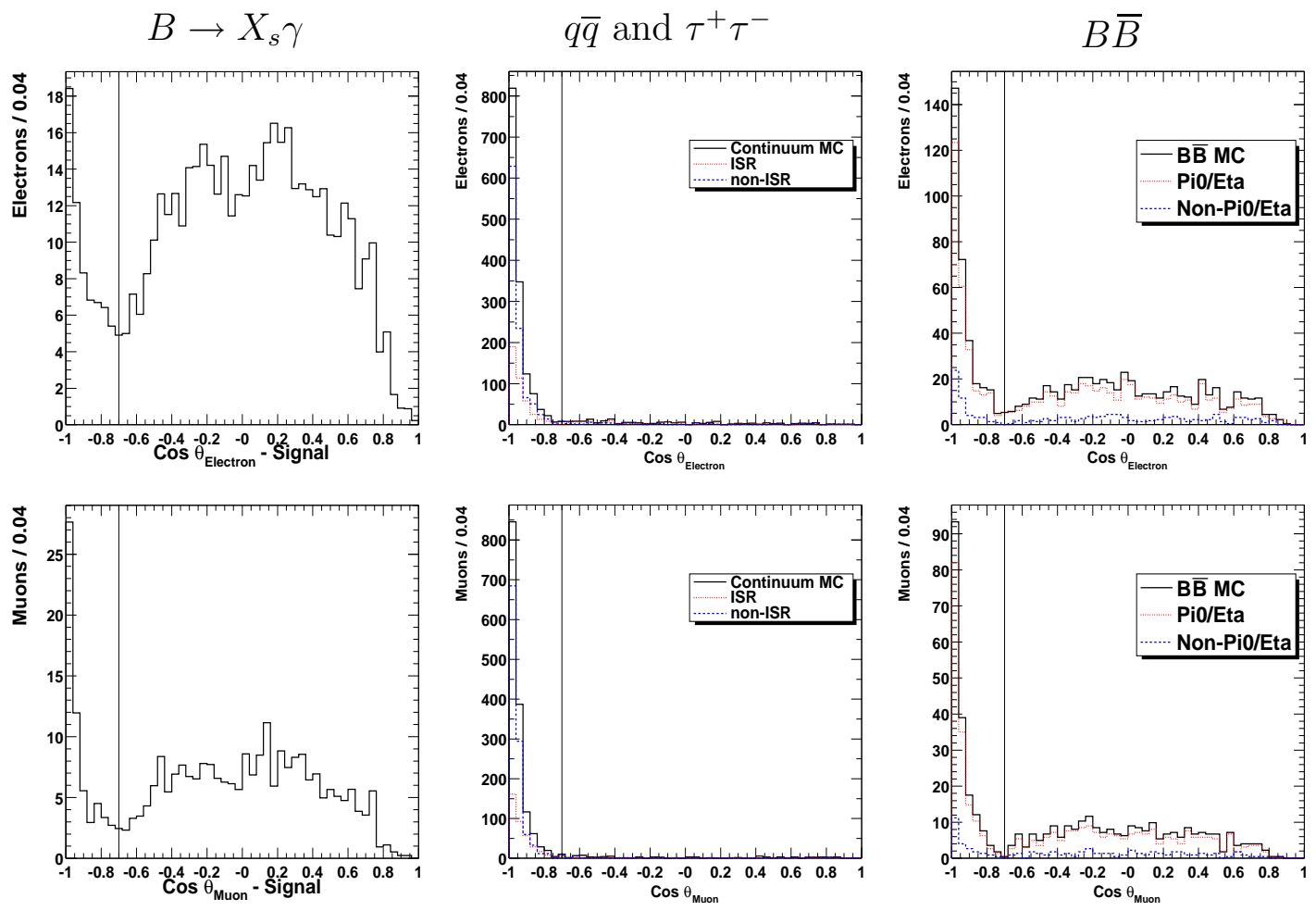

Figure 3.7: The cosine of the angle between the high-energy photon and the tag lepton from MC electron (top) and muon (bottom) candidates. The vertical lines indicate the minimum $\cos \theta_{\gamma l}$ value accepted.

Some commentary is called for as to why these distributions look as they do. For $B \bar{B}$ events, when the photon and the lepton candidates originate from different $B$ mesons, the distribution of $\cos \theta_{\gamma l}$ is expected to be isotropic. However, when they are from the same $B$ meson, they will tend to be aligned, moving away from each other, creating a surplus in the distribution around $\cos \theta_{\gamma l}=-1$. It might be surprising to see this surplus for signal events as well, where a high-momentum lepton has to originate from the other $B$ meson. This is observed particularly for muons and is due to pions from the $X_{s}$ misidentified as lepton candidates.

The remaining features of the $B \bar{B}$ background and signal distributions are shaped by the earlier selection criteria: The region of $\cos \theta_{\gamma l}$ near +1 is depleted by the shower 
isolation requirement. The event shape requirements are more likely to remove events, as the tag direction gets closer to a line along or opposite to the photon direction, since such events would be more jet-like.

The simulated continuum background is seen to peak strongly toward $\cos \theta_{\gamma l}=$ -1 . In the c.m. frame, this would be expected to peak toward $\cos \theta_{\gamma l}= \pm 1$ as a consequence of the jet-like topology. The peak in the forward direction is absent since phase space considerations imply that the probability of two high-momentum particles occurring in the same jet $\left(\cos \theta_{\gamma l}=+1\right)$ is less than the probability of them occurring in different jets $\left(\cos \theta_{\gamma l}=-1\right)$. Moreover, the shower isolation requirement strongly suppresses the $\cos \theta_{\gamma l}=+1$ peak.

After the directional requirement, the main source of lepton candidates in signal events are real leptons from semileptonic decays of the $B$ meson. This motivates a final tagging criterion: $E_{\text {miss }}^{*}>0.8 \mathrm{GeV}$, where $E_{m i s s}^{*}$ is the missing energy in the $\mathrm{cm}$ frame defined as the difference between the energy of the beams and the total energy of all good tracks and photons. Due to the energy of the undetected neutrino, most of the signal events satisfy this criterion. As shown in Figure 3.8, the continuum background events are more likely to fail, since lepton candidates from misidentification and from the decays of the neutral mesons still play an important role.

The composition of the tagged events after these criteria are:

For signal and $B \bar{B}$ events, almost all the tagging leptons arise from the decay $B \rightarrow X_{c} \ell \nu$. The rate of such events are corrected in the Monte Carlo based on independent measurements of the electron spectrum (Section 6.3.2). The fraction of fake tags, i.e. non-lepton charged particles mis-identified as electrons or muons, is very low.

Among the various continuum backgrounds, QED processes have much higher tagging efficiency than $q \bar{q}$ processes. This increases their fraction in total continuum background significantly. For the $q \bar{q}$ events, fake tags, especially fake muon tags from misidentified pions, play an important role (about one-fourth of electron and threefifths of muon tags). Finally, $c \bar{c}$ events are 3.5 times more likely to have a tag than light-quark $q \bar{q}$ events, so they contribute more tagged events, even though they make 

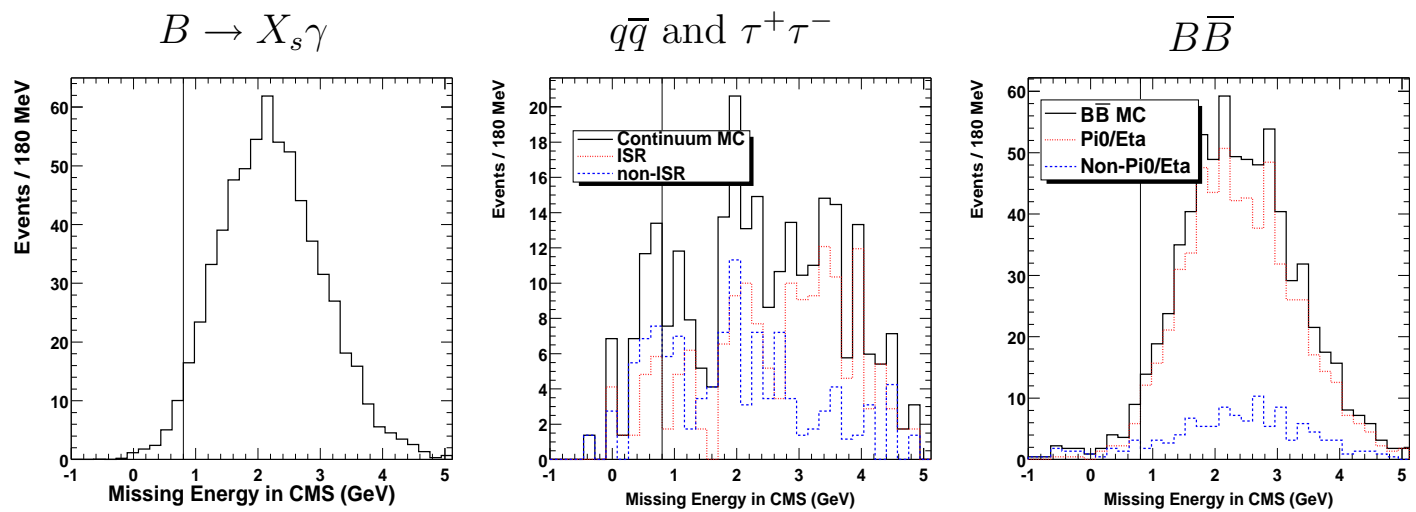

Figure 3.8: The c.m.-frame missing energy distribution (in $\mathrm{GeV}$ ) from $\mathrm{MC}$ signal and background events passing all other requirements. The vertical lines indicate the minimum accepted value.

up only one-third of the total $q \bar{q}$ background before the tagging. Note that although only $5 \%$ of signal events are retained after tagging, the total suppression of the $q \bar{q}$ backgrounds is by a factor of more than 1300 .

\subsection{Optimization of the Selection Criteria}

As mentioned briefly earlier, most of the requirements to be applied have been subjected to an optimization procedure. The goal is to maximize the expected statistical significance of the branching fraction to be measured before the analysis chain is applied to actual data. For this purpose, a figure of merit is defined: $Q=\mathrm{S}^{2} /\left(\mathrm{S}+\mathrm{B}^{\prime}\right)$, where $\mathrm{S}$ and $\mathrm{B}^{\prime}$ are the signal and effective background expectations for an integrated luminosity of $80.5 \mathrm{fb}^{-1}$, as calculated from the selection criteria efficiencies measured in the available Monte Carlo samples listed in Table 2.1.

What separates this figure of merit from the more common $S^{2} /(S+B)$, is the effective background term, B'. This quantity is defined as the sum of the expectations for $B \bar{B}$ and continuum backgrounds, $\mathrm{B}$ and $\mathrm{C}$ respectively, but with the expected continuum events scaled by a factor: $\mathrm{B}^{\prime} \equiv \mathrm{B}+\mathrm{C} /(1-f)$, where $f$ is the fraction of on-resonance running. This scaling is necessary, because the amount of off-resonance 
data, which is used to subtract the remaining continuum backgrounds after the event selection step, is of limited size.

The optimization procedure is iterative. Based on previous measurements ([56] in particular), an initial set of selection criteria is chosen. Then at each step, all criteria are applied to the Monte Carlo signal $\left(K^{*} \gamma\right.$ and $X_{s} \gamma$ using KN480) and background ( $B \bar{B}$ plus continuum) samples, except for the particular selection criterion under study. The extreme value accepted for the variable in question is varied, and the figure of merit plotted (Figure [3.9). When there is a clear maximum in $Q$, the requirement imposed on that variable is set to the maximizing value. If the plotted $Q$ has a broad maximum, the particular value chosen is the one with highest signal efficiency. It is also possible to drop a criterion from the analysis chain, if the maximum for $Q$ indicates so.
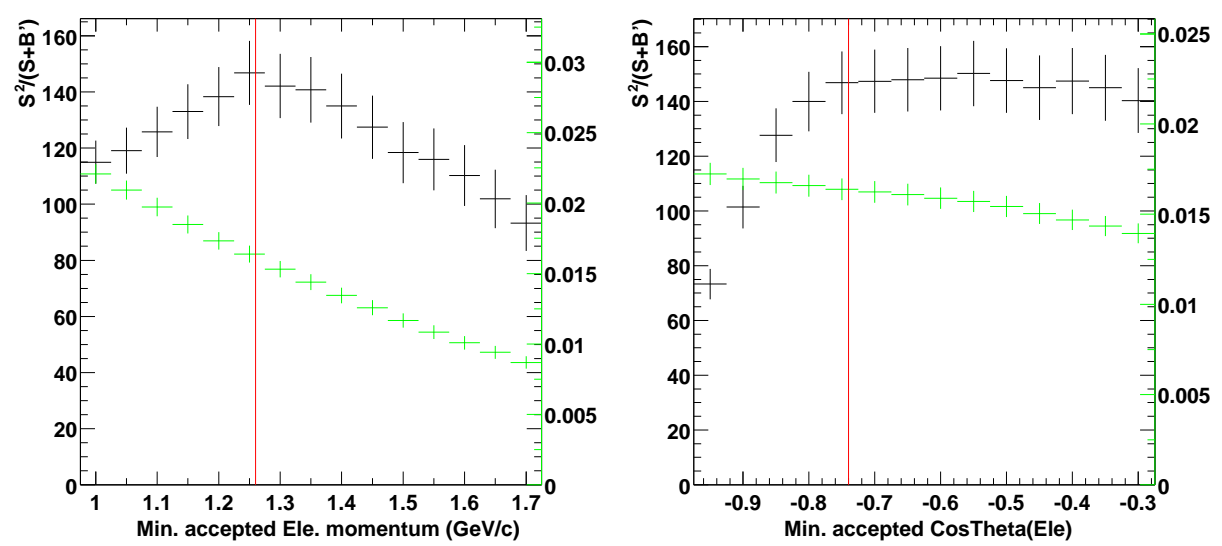

Figure 3.9: Two examples of the optimization plots: for the momentum (left) and angle (right) requirements on the electron tags. Each plot shows how the figure of merit, $Q$ (black points, left scale) and the signal efficiency (green points with smaller errors, right scale) vary with the minimum accepted value, using Monte Carlo samples. For each plot, all other criteria are applied at their nominal values.

Once all the criteria are optimized one-by-one, the cycle is repeated until a stable set of criteria are obtained. This set (except for the requirements on event topology and the related tag quantity of total missing energy) is applied once more to the Monte Carlo samples to obtain the input events used in the computation of the Fisher 
discriminant coefficients (Section 3.5). If there are significant changes in the coefficients, the optimization restarted. Only when the Fisher discriminant coefficients are also stable, the optimization process is terminated, and a final set of verification plots is produced.

For the present analysis, the optimization is performed in the $E_{\gamma}^{*}$ range of 2.0$2.7 \mathrm{GeV}$. The set of quantities subject to this optimization process consists of:

- Minimum distance of shower isolation (Section 3.3.2).

- Minimum energies for second photons used in the $\pi^{0}$ and $\eta$ vetoes, separately for each parent and each $E_{\gamma}^{*}$ range (Table 3.6]).

- Extreme values required for event shape quantities $R_{2}^{*}$ and Fisher discriminant (Section 3.5).

- Required minima on for all tagging variables: Momenta and angles for electrons and muons separately and the total missing energy (Section 3.7).

It is important to note that, apart from this optimization process, certain alternatives have also been studied, in order not to end up with a local maximum of the figure of merit. One such alternative has been mentioned in Section 3.5 the Fisher discrimant requirement has been compared to a method in which individual requirements are applied on the various event topology variables. Another case is studied by dropping the lepton tag criteria: Even with the extra systematic uncertainty introduced because of the corrections to the semileptonic decay rates, a tagged sample has a better performance than an untagged sample.

\subsection{Requirement on Effective Multiplicity}

Optimization process was carried out, as described, using Monte Carlo simulations for signal and background samples. The off-resonance data were not used, not only because of the simulation's superior statistics, but more fundamentally because the off-resonance data are later used to subtract the actual continuum backgrounds. Using those data in the optimization would constitute a direct bias. 
However, it is known that the available simulations of $q \bar{q}$ and $\tau^{+} \tau^{-}$events do not represent all components of the continuum background. In particular, despite the suppression that is provided by the preselection and event topology requirements, a substantial missing contribution from two-photon (and/or QED) processes can be expected. This contribution will be strongly enhanced by the lepton (especially electron) tagging, enough so that it might significantly worsen the statistical precision of the tagged analysis, compared to the precision expected purely from the simulations.

These processes have been studied in the searches for $B^{0} \rightarrow l^{+} l^{-}$decays [65], where they constitute a major background. In that context, it has been determined that a requirement on the minimum effective multiplicity, $N_{\text {mult }}$, defined as the sum of the total number of good tracks plus half the number of good photons with $E>80 \mathrm{MeV}$ reconstructed in the event, provides a good way to reduce the QED continuum backgrounds. It allows a higher efficiency with better rejection than a tighter requirement on the number of tracks only.

Thus a post-optimization criterion on this quantity is adopted as a final step in the event selection. However, it is important to make sure that such a criterion does not introduce extra model-dependence to the signal efficiency. Table 3.8 shows the efficiency vs. $X_{s}$ multiplicity for several choices of a minimum accepted $N_{\text {mult }}$ value. Based on this table, a conservative choice is made: $N_{m u l t} \geq 4.5$. As can be seen in table 3.2 the resulting effects on the overall signal efficiency are very small.

\subsection{Efficiency vs. Photon Energy}

Although the optimization procedure has been global with respect to $E_{\gamma}^{*}$ (the only exception being the separate $\pi^{0}(\eta)$ vetoes for two energy regions), the resulting efficiencies need to be studied as a function of $E_{\gamma}^{*}$ in order to extract the $E_{\gamma}^{*}$ spectrum rather than just the overall $B \rightarrow X_{s} \gamma$ branching fraction. While this study can be carried out with the KN480 Monte Carlo samples, they suffer from depleted statistics toward the edges of the spectrum. Moreover, it is preferable to re-measure the efficiencies on a sample that is independent of the original one utilized for determination of the selection criteria [66]. Therefore, the efficiency figures that are used 


\begin{tabular}{|c|c|c|c|c|}
\hline \multirow{2}{*}{$\begin{array}{c}X_{s} \\
\text { Multiplicity }\end{array}$} & \multirow[b]{2}{*}{ Events } & \multicolumn{3}{|c|}{ Efficiency of $N_{\text {mult }}$ requirement } \\
\hline & & $\geq 4.5$ & $\geq 5.0$ & $\geq 5.5$ \\
\hline 2 & 1091 & 0.994 & 0.968 & 0.914 \\
\hline 3 & 2785 & 0.997 & 0.987 & 0.958 \\
\hline 4 & 2119 & 0.999 & 0.997 & 0.986 \\
\hline 5 & 1516 & 0.999 & 0.999 & 0.995 \\
\hline 6 & 1001 & 1.000 & 0.998 & 0.998 \\
\hline 7 & 570 & 1.000 & 1.000 & 0.998 \\
\hline$\geq 8$ & 544 & 1.000 & 1.000 & 1.000 \\
\hline
\end{tabular}

Table 3.8: Efficiencies of possible choices of an $N_{\text {mult }}$ requirement vs. true $X_{s}$ signal multiplicity, based on summing the "flat" Monte Carlo samples for the two charge states $\left(B^{ \pm}\right.$and $\left.B^{0}\right) . N_{\text {mult }}$ is defined in the text, while $X_{s}$ multiplicity is defined as the total number of charged and neutral kaons and pions (excluding pions from $K_{S}^{0}$ decay) plus $\eta$ mesons which decay to $\gamma \gamma$, according to Monte Carlo truth. Efficiency is relative to a selection including all other cuts.

in the extraction are measured using the flat Monte Carlo samples, weighted by the KN480-model spectrum as detailed in Section 2.7. The $K^{*} \gamma$ component is also studied vs. $E_{\gamma}^{*}$, since the Lorentz boost to the c.m. frame and the resolution effects can result in some events appearing far from the nominal $E_{\gamma}$. The two components are then combined in the proper proportion according to the constant-cutoff column of Table 1.1

The studies are performed in $100 \mathrm{MeV}$ bins in $E_{\gamma}^{*}$. To properly examine the energy dependence, the requirement on the energy range, $2.0<E_{\gamma}^{*}<2.7 \mathrm{GeV}$, is removed. This has the potential of artificially reducing the efficiency since it increases the probability of multiple-candidate events, which are rejected. However, the impact of this on the efficiency is at the level of $0.1 \%$ after the photon selection and event topology criteria have been applied, and therefore is negligible.

The extraction of the signal requires the efficiency in bins of reconstructed $E_{\gamma}^{*}$. But in order to even compute an $E_{\gamma}^{*}$ value, the photon has to be found in the detector. This is enforced by the acceptance criterion at the preselection step: the requirement that a photon of high enough energy is present in the event. Since it is not possible to compute the efficiency of this acceptance as function of reconstructed $E_{\gamma}^{*}$, its true 

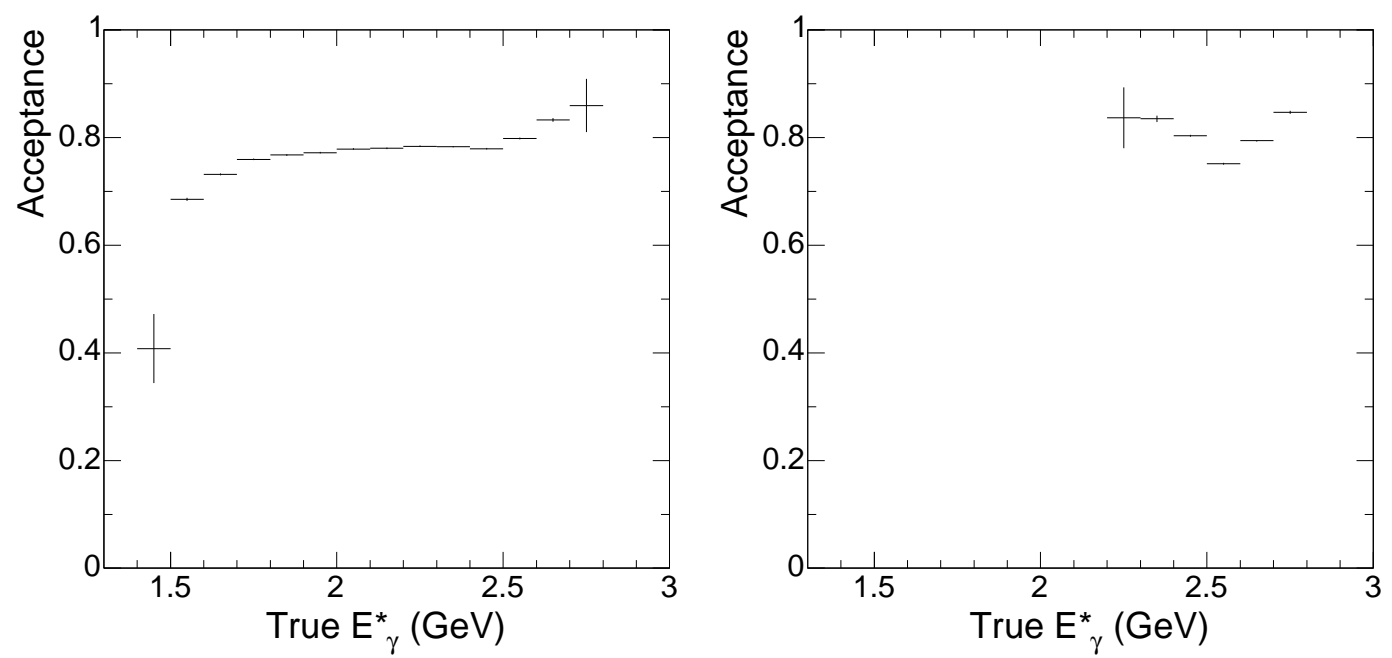

Figure 3.10: Acceptance component of the signal efficiency in bins of true $E_{\gamma}^{*}$, for the KN480-weighted flat $B \rightarrow X_{s} \gamma$ simulation (left) and the $B \rightarrow K^{*} \gamma$ simulation (right). This component includes only the probability of reconstructing a photon with $E_{\gamma}^{*}$ between 1.5 and $3.5 \mathrm{GeV}$, necessarily implying it is in the detector acceptance. For both plots, the $B^{0}$ and $B^{ \pm}$ decays have been added.

(Monte Carlo truth) value has to be used. This is shown in Figure 3.10. For the KN480-weighted flat simulation, the falloff at low values is a reflection of the $1.5 \mathrm{GeV}$ energy minimum, resulting from the low-end tail on reconstructed photon energy resolution. The rise at the highest true $E_{\gamma}^{*}$ (above $2.6 \mathrm{GeV}$ ) has a more subtle source. The $m_{X_{s}}$ cutoff limits $E_{\gamma}$ in the $B$-meson rest frame to $2.525 \mathrm{GeV}$, so that for $E_{\gamma}^{*}$ above this energy the photon must have been emitted forward relative to the $B$ meson direction in the $\Upsilon(4 S)$ frame. In contrast, lower $E_{\gamma}^{*}$ bins are populated by photons emitted in any direction. The polar angle distribution of $B$ mesons from $\Upsilon(4 S)$ decay is proportional to $\sin ^{2} \theta_{B}^{*}$. A photon emitted along the $B$ direction is thus less likely than a random photon to fall into regions (near $\theta^{*}$ of 0 or $\pi$ ) uncovered by the detector. Thus photons with $E_{\gamma}^{*}$ above $2.6 \mathrm{GeV}$ have slightly higher acceptance than the average. Figure 3.10 also shows the acceptance efficiency for $K^{*} \gamma$ events. Here, a similar effect can be observed: photons with $E_{\gamma}^{*}$ appreciably larger or smaller than $E_{\gamma}=2.564 \mathrm{GeV}$ (the $B$-frame photon energy for the central $K^{*}$ mass) tend to be 
emitted respectively along or opposite to the $B$-meson direction in the $\Upsilon(4 S)$ frame, and hence have higher than average acceptance.

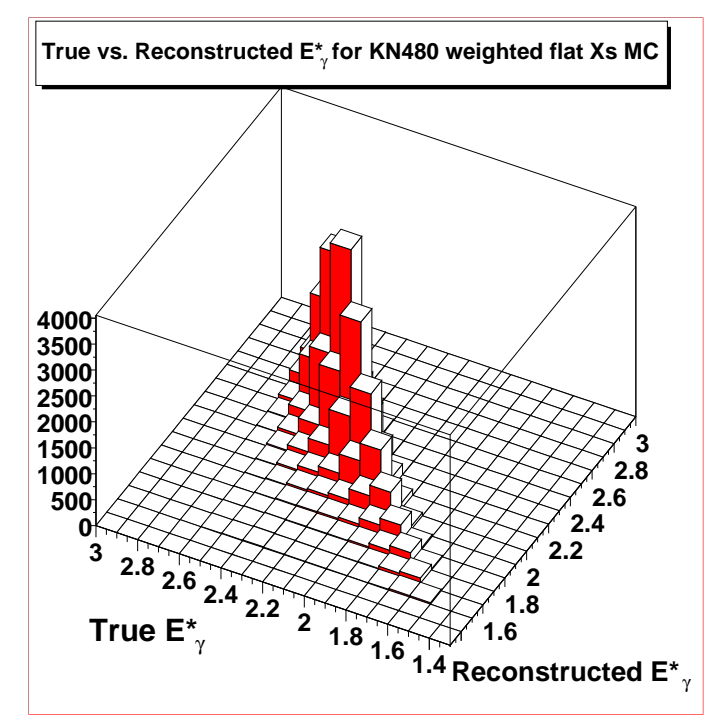

Figure 3.11: True vs. reconstructed $E_{\gamma}^{*}$ correlation plot for the KN480weighted flat Monte Carlo signal simulation, prepared at the acceptance stage, i.e. right after the first preselection criterion.

Figure 3.11 shows the correlation between true and reconstructed $E_{\gamma}^{*}$ for signal photons from events that satisfy the photon presence requirement. Assuming that this correlation is valid for all true signal photons, i.e. that this two-dimensional distribution would have been satisfied by all the signal photons if the detector had $100 \%$ coverage, it is possible to translate the acceptance efficiency from bins of true $E_{\gamma}^{*}$ to bins of reconstructed $E_{\gamma}^{*}$. However, while doing this, it is important to remove the low-end falloff which reflects the $E_{\gamma}^{*}>1.5 \mathrm{GeV}$ minimum. The average acceptance efficiency for true $E_{\gamma}^{*}$ from 2.0 to $2.5 \mathrm{GeV}$ is $78.1 \%$, and is practically constant for the five $100 \mathrm{MeV}$ bins in this energy range (Table 3.9). It is below $2.0 \mathrm{GeV}$ that the falloff seen in Figure 3.10 becomes statistically significant. Hence, in the true-toreconstructed translation, the acceptance efficiency for the $\mathrm{KN}$ component is fixed at $78.1 \%$ for true $E_{\gamma}^{*}$ below $2.0 \mathrm{GeV}$.

Figure 3.12 shows the efficiencies vs. reconstructed $E_{\gamma}^{*}$ after all the requirements, relative to events that satisfy the first preselection criterion. The KN480-weighted flat 

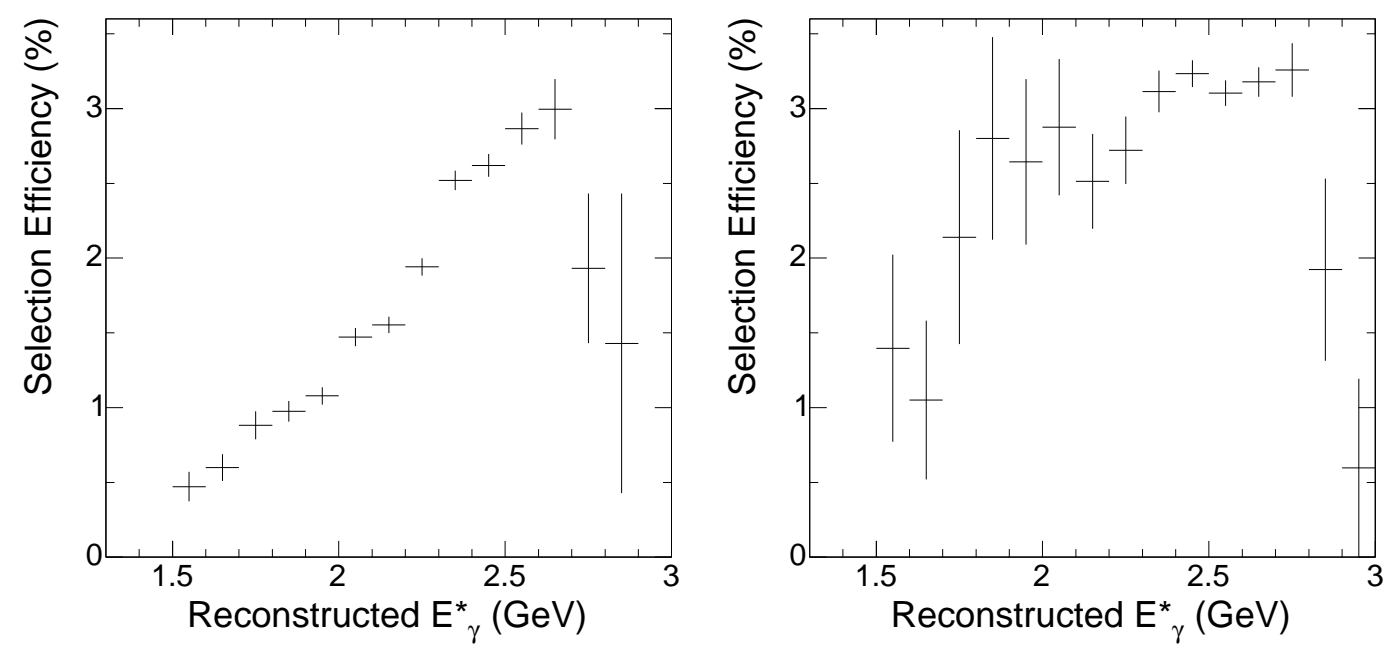

Figure 3.12: Overall selection efficiency for signal in bins of reconstructed $E_{\gamma}^{*}$, for the KN480-weighted flat $B \rightarrow X_{s} \gamma$ simulation (left) and the $B \rightarrow$ $K^{*} \gamma$ simulation (right). This efficiency incorporates all the requirements beyond the first preselection criterion (photon acceptance). For both plots, the two charge states have been added together. Note that nearly all of the $B \rightarrow K^{*} \gamma$ events are contained in the range between 2.3 and $2.8 \mathrm{GeV}$.

MC results and the $K^{*} \gamma$ results are separately shown. (Here as in the previous figure, the two charge states have been combined, each weighted to the same number of $B \bar{B}$ events). The efficiency step at $2.3 \mathrm{GeV}$ is a direct result of the energy-dependent $\pi^{0}(\eta)$ vetoes (Section 3.3.3) - i.e., the choice for reduced efficiency below this energy to better discriminate against backgrounds. But even without that step, a steep rise in efficiency is observed with increasing $E_{\gamma}^{*}$.

The $K^{*}$ component in Figure 3.12 doesn't show the same rise as the KN480 component, and is also slightly higher in some of the region of overlap. However one should keep in mind that the efficiency is at least in part related to the true photon energy. For example, the properties of the remainder of the final state should correlate better with the true energy than with reconstructed energy - which for $K^{*} \gamma$ is much more narrowly confined. Therefore it is not surprising to observe the higher efficiencies for the $K^{*} \gamma$ component. 
With all these necessary components measured, the total efficiency can be calculated. For every reconstructed- $E_{\gamma}^{*}$ bin, the fraction of its contents coming from each true- $E_{\gamma}^{*}$ bin is read off from Figure 3.11. These fractions are multiplied by the acceptance efficiencies from Figure 3.10 (with the caveat about the lower energy bins taken into account), and summed to obtain an acceptance figure for that reconstructed- $E_{\gamma}^{*}$ bin. Finally these are multiplied by the efficiency of the other requirements from Figure 3.12. This process is independently performed for the $X_{s} \gamma$ and $K^{*} \gamma$ components and then combined together. The results are illustrated in Figure 3.13.

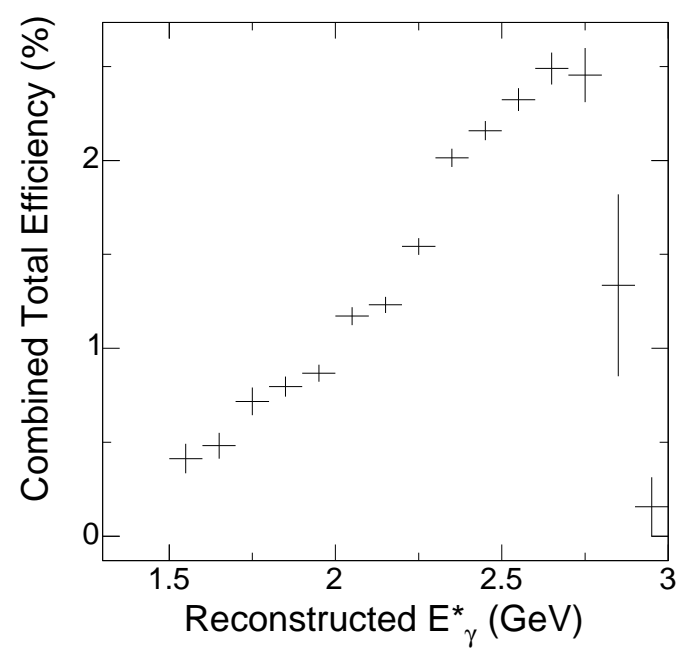

Figure 3.13: Overall efficiency for signal in bins of reconstructed $E_{\gamma}^{*}$, for the default (KN480) signal model. The plot incorporates the effects of all the selection criteria, including the photon acceptance.

Table 3.9 summarizes the step-by-step results in this subsection. It should be noted that all efficiency numbers presented here are efficiencies within a given bin of $E_{\gamma}^{*}$. This is in contrast to the cumulative numbers in Table 3.2, which are relative to the integrated spectra.

It is worth discussing the pronounced rise of selection efficiency with increasing $E_{\gamma}^{*}$. Much of this rise is a consequence of the optimization procedure, and in particular of the computation of the Fisher-discriminant coefficients. Over the range from 2.0 to $2.7 \mathrm{GeV}$, the background input sample (continuum background) to the computation 


\begin{tabular}{|c|c|c|c|c|c|c||c|}
\hline \multirow{2}{*}{$\begin{array}{c}E_{\gamma}^{*} \\
(\mathrm{GeV})\end{array}$} & \multicolumn{2}{|c|}{ Acceptance } & \multicolumn{2}{|c|}{ Selection Criteria } & \multicolumn{3}{c|}{ Total } \\
\cline { 2 - 7 } & $X_{s} \gamma$ & $K^{*} \gamma$ & $X_{s} \gamma$ & $K^{*} \gamma$ & $X_{s} \gamma$ & $K^{*} \gamma$ & Combined \\
\hline \hline $1.5-1.6$ & $68.5 \pm 0.3$ & $0.0 \pm 0.0$ & $0.47 \pm 0.10$ & $1.40 \pm 0.63$ & $0.37 \pm 0.08$ & $1.10 \pm 0.49$ & $0.41 \pm 0.08$ \\
$1.6-1.7$ & $73.1 \pm 0.2$ & $0.0 \pm 0.0$ & $0.60 \pm 0.09$ & $1.05 \pm 0.53$ & $0.47 \pm 0.07$ & $0.83 \pm 0.42$ & $0.48 \pm 0.07$ \\
$1.7-1.8$ & $75.9 \pm 0.1$ & $0.0 \pm 0.0$ & $0.88 \pm 0.09$ & $2.14 \pm 0.72$ & $0.69 \pm 0.07$ & $1.69 \pm 0.57$ & $0.72 \pm 0.07$ \\
$1.8-1.9$ & $76.7 \pm 0.1$ & $0.0 \pm 0.0$ & $0.98 \pm 0.07$ & $2.80 \pm 0.68$ & $0.76 \pm 0.05$ & $2.20 \pm 0.54$ & $0.80 \pm 0.05$ \\
$1.9-2.0$ & $77.2 \pm 0.1$ & $0.0 \pm 0.0$ & $1.08 \pm 0.06$ & $2.64 \pm 0.55$ & $0.84 \pm 0.05$ & $2.08 \pm 0.44$ & $0.87 \pm 0.05$ \\
$2.0-2.1$ & $77.9 \pm 0.1$ & $0.0 \pm 0.0$ & $1.47 \pm 0.06$ & $2.88 \pm 0.46$ & $1.15 \pm 0.05$ & $2.27 \pm 0.36$ & $1.17 \pm 0.05$ \\
$2.1-2.2$ & $78.0 \pm 0.1$ & $0.0 \pm 0.0$ & $1.55 \pm 0.06$ & $2.51 \pm 0.32$ & $1.21 \pm 0.04$ & $1.99 \pm 0.25$ & $1.23 \pm 0.04$ \\
$2.2-2.3$ & $78.4 \pm 0.1$ & $83.6 \pm 5.7$ & $1.94 \pm 0.06$ & $2.72 \pm 0.23$ & $1.52 \pm 0.05$ & $2.16 \pm 0.18$ & $1.54 \pm 0.05$ \\
$2.3-2.4$ & $78.3 \pm 0.1$ & $83.5 \pm 0.6$ & $2.52 \pm 0.07$ & $3.11 \pm 0.14$ & $1.97 \pm 0.05$ & $2.48 \pm 0.11$ & $2.01 \pm 0.05$ \\
$2.4-2.5$ & $77.9 \pm 0.1$ & $80.3 \pm 0.2$ & $2.62 \pm 0.08$ & $3.23 \pm 0.09$ & $2.06 \pm 0.06$ & $2.55 \pm 0.07$ & $2.16 \pm 0.05$ \\
$2.5-2.6$ & $79.8 \pm 0.2$ & $75.1 \pm 0.2$ & $2.87 \pm 0.11$ & $3.10 \pm 0.09$ & $2.29 \pm 0.09$ & $2.40 \pm 0.07$ & $2.32 \pm 0.06$ \\
$2.6-2.7$ & $83.3 \pm 0.3$ & $79.4 \pm 0.2$ & $3.00 \pm 0.20$ & $3.18 \pm 0.10$ & $2.45 \pm 0.17$ & $2.52 \pm 0.08$ & $2.49 \pm 0.08$ \\
$2.7-2.8$ & $86.1 \pm 4.9$ & $84.7 \pm 0.3$ & $1.93 \pm 0.50$ & $3.26 \pm 0.18$ & $1.58 \pm 0.41$ & $2.67 \pm 0.15$ & $2.45 \pm 0.14$ \\
\hline $2.0-2.7$ & $78.5 \pm 0.1$ & $78.2 \pm 0.1$ & $2.25 \pm 0.03$ & $3.13 \pm 0.05$ & $1.77 \pm 0.02$ & $2.46 \pm 0.04$ & $1.88 \pm 0.02$ \\
\hline
\end{tabular}

Table 3.9: Efficiencies (in percent) for $X_{s} \gamma$ and $K^{*} \gamma$ in bins of $E_{\gamma}^{*}$. The acceptance is given as a function of true $E_{\gamma}^{*}$, whereas all the other efficiencies are given as functions of reconstructed $E_{\gamma}^{*}$. In the calculation of "Total" efficiencies, acceptance values are overwritten in the 1.5 to $2.0 \mathrm{GeV}$ range for $X_{s} \gamma$ by $78.1 \% \pm 0.2 \%$ (see text). "Combined" numbers have been computed by weighting the $X_{s} \gamma$ and $K^{*} \gamma$ components according to the default (KN480) signal model (Section [1.8). 
falls by nearly a factor of two, while the signal input sample peaks above $2.3 \mathrm{GeV}$ and is small at the ends of the interval. Thus the background has a much larger fraction of events in the low- $E_{\gamma}^{*}$ region than does the signal. Although the energy of the photon itself is not an input parameter to the discriminant, adding up all the 18 energy cones provides enough information to cause a strong correlation between the value of the Fisher discriminant and $E_{\gamma}^{*}$. Fortunately, this correlation appears to be with the photon energy itself, rather than with the composition of the $X_{s}$ system; systematic studies showing this are described in Section 6.2, where a systematic uncertainty for possible sensitivity to variations in $X_{s}$ fragmentation is assigned. A variation of efficiency purely with photon energy is largely benign.

Finally, it must be noted that the $2.0-2.7 \mathrm{GeV}$ results in this section are slightly different from the efficiencies listed in Table 3.2. The main reason for the difference is that the corrections to the semileptonic decays, which will be discussed later in Section 6.3.2, have now been taken into account. These corrections, applied to the lepton tagging side of the event, increase the signal efficiency by about $8 \%$ and are independent of the photon $E_{\gamma}^{*}$.

\subsection{Expectated Signal and Backgrounds after Se- lection}

Table 3.10 gives the expected number of signal and background events in $100 \mathrm{MeV}$ energy bins after all requirements except the photon energy range selection of 2.0$2.7 \mathrm{GeV}$ have been applied. The figure of merit, $Q$, is also tabulated for each of the energy bins. If these predictions were true and complete, it would be possible to extract the $B \rightarrow X_{s} \gamma$ branching fraction to a statistical precision of $\approx 8 \%$. However, this will not be the case, since the background simulations do not include the QED continuum contributions and the expected number of signal events can be significantly different from the expections derived from the KN480 model. Experience from earlier measurements suggest that these two effects are likely to be in opposite directions. It is important to note that whatever the outcome, once set, the selection criteria given 
in the chapter are not modified for the rest of the analysis. (The only exception is the selection on the photon energy range, which is revisited when the Monte Carlo estimates for the systematic errors are also available.)

Figure 3.14 shows the expected signal and background spectra.

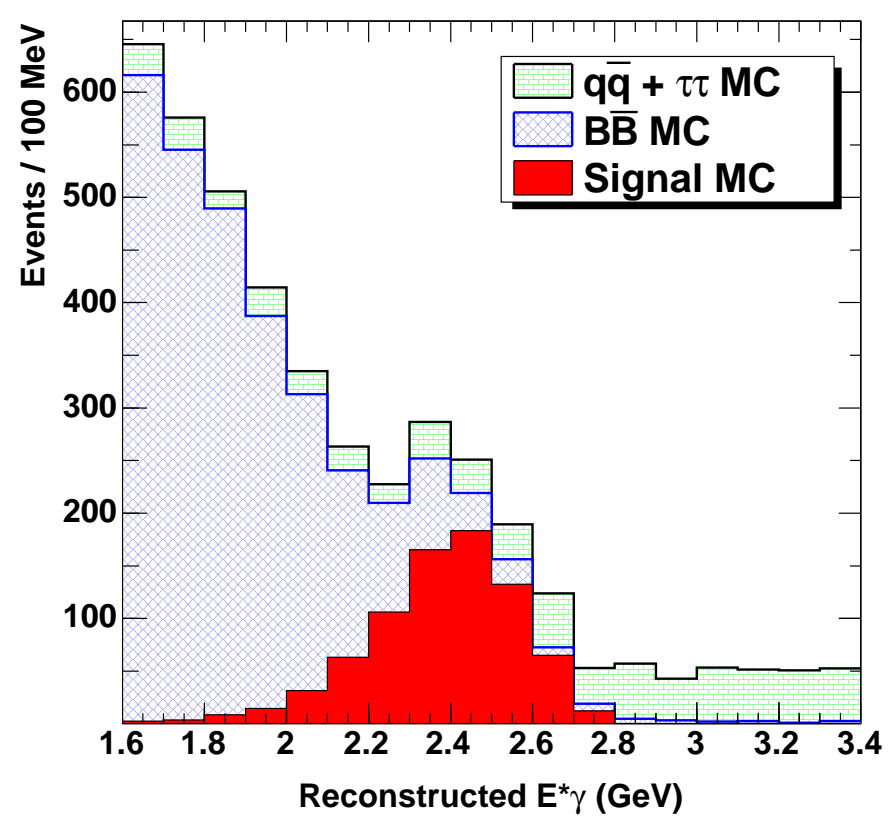

Figure 3.14: The simulated $E_{\gamma}^{*}$ distribution of signal, continuum ( $q \bar{q}$ and $\tau^{+} \tau^{-}$) background and $B \bar{B}$ background events after all selection criteria (except that on $E_{\gamma}^{*}$ itself) have been applied. 


\begin{tabular}{|c|c|c|c|c|}
\hline$E_{\gamma}^{*}(\mathrm{GeV})$ & $q \bar{q}$ and $\tau^{+} \tau^{-}$ & $B \bar{B}$ & Signal & $Q$ \\
\hline $1.6-1.7$ & $27.2 \pm 6.5$ & $590.4 \pm 16.2$ & $2.3 \pm 0.7$ & $0.0 \pm 0.0$ \\
$1.7-1.8$ & $31.5 \pm 6.8$ & $495.0 \pm 14.9$ & $3.5 \pm 0.9$ & $0.0 \pm 0.0$ \\
$1.8-1.9$ & $16.3 \pm 4.9$ & $442.9 \pm 14.0$ & $8.6 \pm 1.4$ & $0.1 \pm 0.0$ \\
$1.9-2.0$ & $26.9 \pm 6.4$ & $318.1 \pm 11.9$ & $14.8 \pm 1.8$ & $0.4 \pm 0.1$ \\
$2.0-2.1$ & $28.8 \pm 6.5$ & $266.2 \pm 10.7$ & $33.7 \pm 2.8$ & $2.0 \pm 0.4$ \\
$2.1-2.2$ & $28.8 \pm 6.7$ & $161.6 \pm 8.4$ & $62.4 \pm 3.8$ & $7.8 \pm 1.3$ \\
$2.2-2.3$ & $19.8 \pm 5.5$ & $86.6 \pm 6.1$ & $108.2 \pm 4.9$ & $30.5 \pm 4.9$ \\
$2.3-2.4$ & $35.3 \pm 7.4$ & $82.4 \pm 6.0$ & $171.8 \pm 6.0$ & $50.0 \pm 6.7$ \\
$2.4-2.5$ & $40.7 \pm 8.0$ & $32.9 \pm 3.8$ & $182.7 \pm 5.8$ & $55.4 \pm 7.6$ \\
$2.5-2.6$ & $30.3 \pm 6.8$ & $23.8 \pm 3.2$ & $132.4 \pm 4.6$ & $39.5 \pm 6.2$ \\
$2.6-2.7$ & $47.4 \pm 8.6$ & $7.8 \pm 1.8$ & $67.9 \pm 2.9$ & $8.8 \pm 1.5$ \\
$2.7-2.8$ & $36.4 \pm 7.5$ & $6.7 \pm 1.7$ & $12.3 \pm 0.7$ & $0.4 \pm 0.1$ \\
$2.8-2.9$ & $52.2 \pm 9.1$ & $4.5 \pm 1.4$ & $0.3 \pm 0.1$ & $0.0 \pm 0.0$ \\
$2.9-3.0$ & $39.8 \pm 7.9$ & $2.7 \pm 1.1$ & $0.0 \pm 0.0$ & $0.0 \pm 0.0$ \\
$3.0-3.1$ & $51.3 \pm 8.9$ & $2.2 \pm 1.0$ & $0.0 \pm 0.0$ & $0.0 \pm 0.0$ \\
$3.1-3.2$ & $48.9 \pm 8.8$ & $2.2 \pm 1.0$ & $0.0 \pm 0.0$ & $0.0 \pm 0.0$ \\
$3.2-3.3$ & $51.2 \pm 9.0$ & $0.9 \pm 0.6$ & $0.0 \pm 0.0$ & $0.0 \pm 0.0$ \\
$3.3-3.4$ & $54.6 \pm 9.1$ & $0.9 \pm 0.6$ & $0.0 \pm 0.0$ & $0.0 \pm 0.0$ \\
$3.4-3.5$ & $25.3 \pm 6.2$ & $0.9 \pm 0.6$ & $0.0 \pm 0.0$ & $0.0 \pm 0.0$ \\
\hline $2.0-2.7$ & $231.1 \pm 18.9$ & $661.3 \pm 16.9$ & $759.1 \pm 12.1$ & $159.4 \pm 9.2$ \\
\hline
\end{tabular}

Table 3.10: Expectated number of signal and background events based on Monte Carlo simulation, in $80.5 \mathrm{fb}^{-1}$ in bins of $E_{\gamma}^{*}$ after all selection criteria (except that on $E_{\gamma}^{*}$ itself) have been applied. Errors listed are purely from Monte Carlo statistics. $Q=\mathrm{S}^{2} /\left(\mathrm{S}+\mathrm{B}^{\prime}\right)$ is the statistical figure of merit for each bin (used in the $2.0<E_{\gamma}^{*}<2.7 \mathrm{GeV}$ range for selection optimization), as defined in section 3.8 . 


\section{Chapter 4}

\section{$B \bar{B}$ Background}

After the event selection criteria are applied, the continuum backgrounds are highly suppressed. The remaining contribution from the continuum backgrounds can be subtracted using the off-resonance continuum data. However, the contribution from the $B \bar{B}$ backgrounds, now dominant at low $E_{\gamma}^{*}$, has to be predicted using the Monte Carlo simulations.

Although the event and detector simulation is quite detailed and is more than adequate for most studies of exclusive decays, the precision desired for performing a subtraction in an inclusive analysis requires a detailed study. Therefore, in the second stage of the analysis, the sources of the $B \bar{B}$ backgrounds are studied in independent control samples in order to correct the Monte Carlo predictions.

It should be emphasized that throughout these studies the signal region of the photon spectrum is blinded in data. All the corrections are derived and tested with absolutely no input from the signal candidates that satisfy the signal selection criteria of Chapter 3] so as to avoid experimenter biases as much as possible.

\subsection{Components of the $B \bar{B}$ Backgrounds}

The $B \bar{B}$ backgrounds consist mostly of photons from energetic $\pi^{0}$ and $\eta$ decays but also contain sizeable components from other sources. A full breakdown of these sources is given in Table 4.1. In order to provide more meaningful statistics, the 
composition is shown for untagged samples, i.e. before the lepton tagging requirements have been applied. The lepton tagging is unlikely to lead to any significant changes in the relative fractions of the different contributions.

\begin{tabular}{|l|l|c|c|}
\hline \multicolumn{2}{|c|}{ Particle } & $2.0<E_{\gamma}^{*}<2.7 \mathrm{GeV}$ & $1.5<E_{\gamma}^{*}<1.9 \mathrm{GeV}$ \\
\cline { 1 - 3 } Truth-match & Parentage & $37,192 \mathrm{MC}$ events & $137,693 \mathrm{MC}$ events \\
\hline Photon & $\pi^{0}$ & 0.640 & 0.607 \\
& $\eta$ & 0.174 & 0.185 \\
& $\omega$ & 0.024 & 0.040 \\
& $\eta^{\prime}$ & 0.011 & 0.012 \\
& $B$ & 0.007 & 0.024 \\
& $J / \psi$ & 0.008 & 0.005 \\
& Other & 0.001 & 0.001 \\
\cline { 2 - 4 } & Total & 0.864 & 0.874 \\
\hline$\pi^{0}$ (merged) & 0.001 & 0.001 \\
$e^{ \pm}$ & 0.036 & 0.091 \\
$\bar{n}(n)$ & 0.077 & 0.023 \\
$\bar{p}(p)$ & 0.005 & 0.002 \\
$K_{L}^{0}$ & 0.001 & 0.003 \\
$\pi^{ \pm}$or $K^{ \pm}$ & 0.001 & 0.001 \\
\hline Unmatched & 0.015 & 0.004 \\
\hline
\end{tabular}

Table 4.1: Breakdown of $B \bar{B}$ backgrounds by the origin of the photon candidate using truth-matching. When the candidate is matched to a true photon, the MC parent for the photon is also given. The fraction of each component is given with respect to the total number of $B \bar{B}$ background events in the combined $B^{0} \bar{B}^{0}$ and $B^{+} B^{-}$MC samples before the lepton tagging requirements, for two ranges of energy: The $B \rightarrow X_{s} \gamma$ signal region of $2.0<E_{\gamma}^{*}<2.7 \mathrm{GeV}$ and the lower-energy $B \bar{B}$ background control region. The only $B$ decays in the simulation that yield prompt photons are radiative semileptonic decays. Unmatched entries are due to real machine and cosmic background particles mixed into the generated $B \bar{B}$ events during the MC production.

The table lists the composition in two distinct energy ranges; the $B \rightarrow X_{s} \gamma$ signal range of $2.0<E_{\gamma}^{*}<2.7 \mathrm{GeV}$ and the $B \bar{B}$-background control region below $1.9 \mathrm{GeV}$. Almost all the events in this low-energy region are from $B \bar{B}$ backgrounds, with very small contribution expected from the $B \rightarrow X_{s} \gamma$ signal. Therefore this region is an 
ideal testing ground to compare the corrected $B \bar{B}$ simulation to the data. The results of this comparison are summarized at the end of this chapter, after each of the various components have been studied and corrected.

\subsection{Background from $\pi^{0}$ and $\eta$ Decays}

The method to correct the $\pi^{0}(\eta)$ component relies on measuring the energy spectrum of $\pi^{0}$ and $\eta$ mesons in simulated $B \bar{B}$ events and comparing it to the data. Possible differences can arise from the simulation deficiencies in the production rate or in the variables that are used to select the events. For the results of the comparison to be directly applicable to the $B \rightarrow X_{s} \gamma$ analysis, $B \bar{B}$ events, from which the energy spectra are extracted, should be subjected to essentially the same selection criteria as described in chapter [3, with the obvious exception of the $\pi^{0}$ and $\eta$ vetoes. Small systematic differences in the veto efficiencies are later studied (Section 6.1.2).

The energy spectrum is extracted by measuring the yields in bins of $\pi^{0}(\eta)$ energy. The yields are determined by fitting the mass distributions separately for on-resonance and off-resonance data and for $B \bar{B}$ MC simulated events. Correction factors are then determined by comparing the $B \bar{B}$ MC yields to the difference in the on-resonance and off-resonance data yields. These correction factors are directly applicable to the $B \bar{B}$ Monte Carlo events in the $B \rightarrow X_{s} \gamma$ analysis.

\subsubsection{Selecting Samples of Inclusive $\pi^{0}(\eta)$ Events}

As mentioned above, the selection criteria applied to the $B \bar{B}$ events from which the yields are extracted should be essentially the same as the $B \rightarrow X_{s} \gamma$ analysis. However, the requirements are slightly modified in order to enrich the sample in $\pi^{0}(\eta)$ mesons. These modifications are mostly related to the identification of the $\pi^{0}(\eta)$ mesons, which is well-modeled in the simulation as verified by independent checks, and thus do not compromise the direct applicability of the final correction factors to the $B \rightarrow X_{s} \gamma$ analysis.

The first modification is the lowering of the minimum energy requirement on the 
high-energy photon, which is now considered to be the primary photon candidate from a $\pi^{0}(\eta)$ meson, to $E_{\gamma}^{*}>1.0 \mathrm{GeV}$. Since the meson yields are determined as a function of the $\pi^{0}(\eta)$ energy, lowering the photon energy threshold gives access to more symmetric $\pi^{0}(\eta)$ decays, thus providing a significant increase in statistics.

This primary photon is combined with the other good photons in the event to reconstruct $\pi^{0}$ and $\eta$ candidates. These candidates are subject to the requirements listed in Table 4.2. The requirements on the mass of the photon pair are chosen to be loose enough to allow sufficiently wide sidebands to permit fitting the $\gamma \gamma$ mass spectrum.

\begin{tabular}{|c|c|}
\hline \multirow[b]{2}{*}{ Parameter } & Criterion \\
\hline & For $\pi^{0}$ candidates \\
\hline c.m.-energy of primary photon & $E_{\gamma}^{*}>1 \mathrm{GeV}$ \\
\hline Energy of $2^{\text {nd }}$ photon & $E_{\gamma 2}>30 \mathrm{MeV}$ \\
\hline Polar angle of $2^{\text {nd }}$ photon & $-0.74<\cos \theta_{\gamma 2}<0.93$ \\
\hline Second moment of $2^{\text {nd }}$ photon & 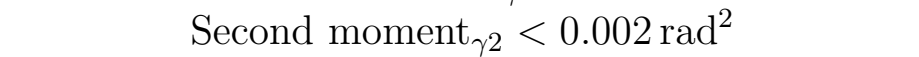 \\
\hline Lateral moment of $2^{\text {nd }}$ photon & $L A T_{\gamma 2}<0.55$ \\
\hline $\begin{array}{l}\text { Mass of } \gamma \gamma \text { combination } \\
\text { c.m.-energy of } \gamma \gamma \text { combination }\end{array}$ & $\begin{array}{c}50<m_{\gamma \gamma}<250 \mathrm{MeV} / c^{2} \quad 400<m_{\gamma \gamma}<700 \mathrm{MeV} / c^{2} \\
1.0<E_{\gamma \gamma}^{*}<3.5 \mathrm{GeV}\end{array}$ \\
\hline
\end{tabular}

Table 4.2: The selection criteria to be satisfied by $\pi^{0}$ and $\eta$ candidates that are used for deriving correction factors for $B \bar{B}$ background simulation.

Once a candidate meson is identified in an event, all the regular selection criteria except the vetoes are applied. However, the statistics of the sample are very limited after the lepton tagging. To improve them, the requirement on the direction of the tag lepton with respect to the high-energy photon is waived and the minimum momentum requirement is loosened: $p_{e}^{*}>1.0 \mathrm{GeV} / c$ and $p_{\mu}^{*}>1.1 \mathrm{GeV} / c$. The effects of this loosening on the derived $\pi^{0}(\eta)$ correction factors will later be addressed in the context of semileptonic corrections to the tagging rate (Section 6.3.4).

\subsubsection{Yields from $\pi^{0}$ Mass Distributions}

In order to extract the $\pi^{0}$ energy spectrum, the $\pi^{0}$ candidates from the above selected data and simulation samples are binned into eight subsamples based on their c.m. 
energy: Seven bins of $200 \mathrm{MeV}$ width for $1.0 \mathrm{GeV}<E_{\pi^{0}}^{*}<2.4 \mathrm{GeV}$ and one wider bin for $2.4 \mathrm{GeV}<E_{\pi^{0}}^{*}<3.0 \mathrm{GeV}$, where the $B \bar{B}$ backgrounds are very low. In each of these bins, a separate fit to the mass distribution is performed. Each of these fits have a component for real $\pi^{0} \mathrm{~s}$ and another component to take into account the random combinations of photons.

For real $\pi^{0}$ decays, the mass distribution has a Gaussian shape, but with extended tails on both ends of the spectrum. These tails are due to the leakage in the calorimeter and the method used to calibrate the energy of the showers to account for it. Therefore to characterize the real- $\pi^{0}$ component of the fit, a modified Crystal Ball (CB) function is used 68]. It consists of the sum of two Gaussian functions above a certain mass threshold and a power law tail below the threshold:

$$
f_{\pi^{0}}(m)= \begin{cases}A_{g}\left[f_{1} G\left(m, \mu_{1}, \sigma_{1}\right)+\left(1-f_{1}\right) G\left(m, \mu_{2}, \kappa \sigma_{1}\right)\right] & \text { for } m>m_{0} \\ A_{p}\left[\frac{p \sigma_{1} / \lambda}{\left(m_{0}-m\right)+p \sigma_{1} / \lambda}\right]^{p} & \text { for } m<m_{0}\end{cases}
$$

where $m_{0} \equiv \mu_{1}-\lambda \sigma_{1}$ is the mass threshold. $G(m, \mu, \sigma)$ is a Gaussian function with mean $\mu$ and width $\sigma$, normalized over the range $m_{0}-\infty$. The first Gaussian function, $G\left(m, \mu_{1}, \sigma_{1}\right)$, is defined as the narrower of the two, with the second one, $G\left(m, \mu_{2}, \kappa \sigma_{1}\right)$, meant to account for the higher-end tail of the mass distribution. The value of $A_{p}$ is fixed by imposing that the function be continuous at $m_{0}$.

Therefore to describe the distribution of real $\pi^{0}$ mesons, there are a total of eight parameters to be determined: $A_{g}, f_{1}, \mu_{1}, \sigma_{1}, \mu_{2}, \kappa, p$ and $\lambda$. Additional parameters are needed for the distribution of the random $\gamma \gamma$ combinations. Unfortunately, given the size of the samples to be fit, it is not possible to determine all of these parameters in a single step. Therefore a strategy of three successive fits is followed, with each step having fewer parameters than the previous one.

Initially, the mass distribution of truth-matched $\pi^{0}$ candidates in the $B \bar{B}$ simulation are fit allowing all parameters but two to vary in the fit (Figure 4.1). The two that are not left free are $\mu_{2}$ and $\kappa$, which are fixed to apriori values of $-0.06 \mathrm{GeV} / c^{2}$ and 9.4, respectively. The fit is not very sensitive to the exact values of these parameters since the wider Gaussian function is essentially a small extension of the narrow 
one, added to improve the quality of the fit on the high-end side of the $\pi^{0}$ mass distribution. However, it is important to note that the $\sigma_{1}$ is still a free parameter and the widths of both Gaussian functions will depend on it. The performance of the fit is validated by comparing the integral of the fitted function to the known number of truth-matched candidates. Table 4.3 shows that the yield from the fit is in very good agreement with the true number of $\pi^{0}$ mesons.

\begin{tabular}{|c|c|c|c|}
\hline \multirow{2}{*}{$\begin{array}{c}E_{\pi^{0}}^{*} \text { bin } \\
(\mathrm{GeV})\end{array}$} & \multicolumn{2}{|c|}{ Number of $\pi^{0}$ candidates } & \multirow{2}{*}{$\begin{array}{c}\text { Ratio } \\
\text { (Fit/True) }\end{array}$} \\
\hline & True & Fitted & \\
\hline $1.0-1.2$ & 69422 & $69386 \pm 629$ & $0.999 \pm 0.009$ \\
\hline $1.2-1.4$ & 143776 & $143676 \pm 821$ & $0.999 \pm 0.006$ \\
\hline $1.4-1.6$ & 124083 & $123980 \pm 560$ & $0.999 \pm 0.005$ \\
\hline $1.6-1.8$ & 96205 & $96011 \pm 435$ & $0.998 \pm 0.005$ \\
\hline $1.8-2.0$ & 72102 & $71923 \pm 476$ & $0.998 \pm 0.007$ \\
\hline $2.0-2.2$ & 46232 & $46066 \pm 373$ & $0.996 \pm 0.008$ \\
\hline $2.2-2.4$ & 13961 & $13917 \pm 153$ & $0.997 \pm 0.011$ \\
\hline $2.4-3.0$ & 2985 & $2949 \pm 64$ & $0.988 \pm 0.022$ \\
\hline
\end{tabular}

Table 4.3: Validation of $\pi^{0}$ mass fits based on Monte Carlo events. The table lists the true and fitted number of $\pi^{0}$ decays, and their ratio, from a sample of truth-matched $\pi^{0}$ mesons, i.e. without the background from random combinatorics.

Along with $\mu_{2}$ and $\kappa$, the parameters $f_{1}$, the relative number of events in the narrow Gaussian, and $\lambda$, the parameter that governs the position of the threshold mass, can be considered as intrinsic parameters of the function $f_{\pi^{0}}$. They relate various components of the lineshape to one another, so that the resulting lineshape adequately describes the $\pi^{0}$ decay process. Therefore, these four parameters are not expected to be different in data and simulation and are fixed for the second step of fits.

The second set of fits is performed to determine the parameters $\mu_{1}, \sigma_{1}$ and $p$. These parameters can be very different for data and simulation, since they describe the precision of the $\mathrm{m}_{\gamma \gamma}$ measurement. However, for the same reason, their values are essentially independent of what sort of event the $\pi^{0}$ candidates originate from: whether the lepton tagging requirements are applied or not, or whether they are from 

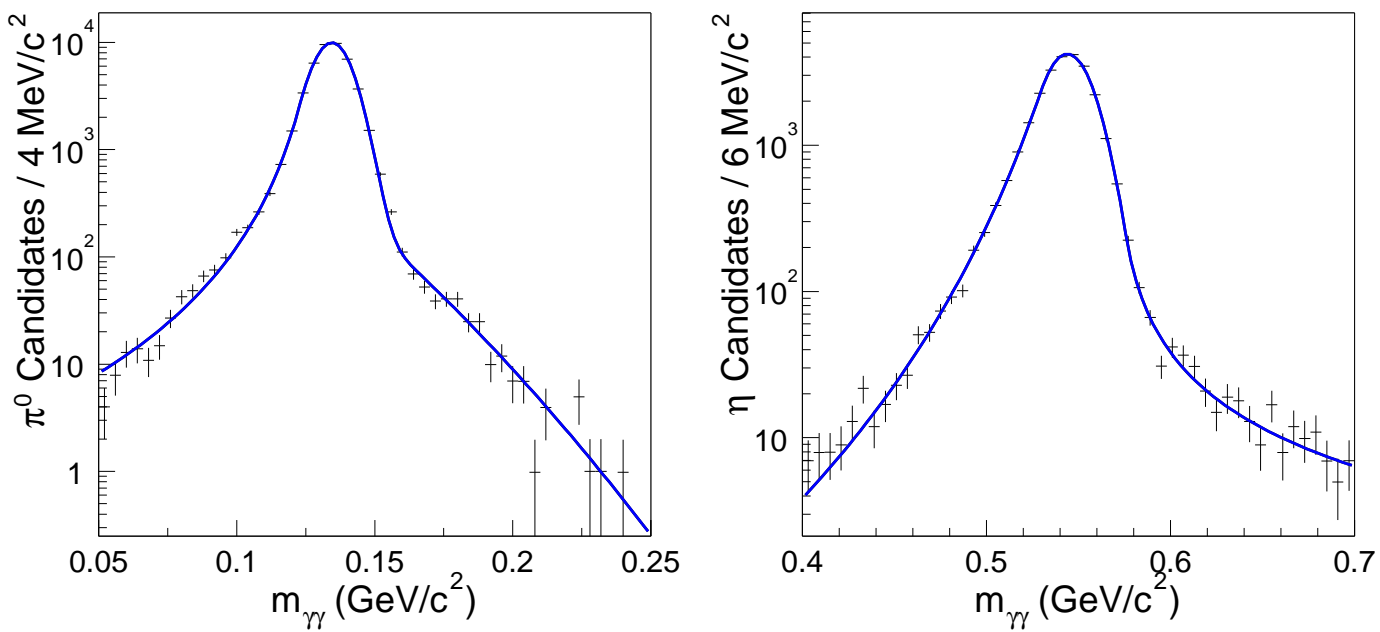

Figure 4.1: Typical mass fits for truth-match Monte Carlo candidates. The left plot shows the results for the $\pi^{0}$ mass fit for $E_{\pi^{0}}^{*}$ bin of $2.0-2.2 \mathrm{GeV}$. The right plot shows the $\eta$ fit results for $E_{\eta}^{*}$ bin of $1.9-2.2 \mathrm{GeV}$.

on-resonance or off-resonance running, there should be only one set of $\mu_{1}, \sigma_{1}$ and $p$ for data and one set for simulation per each bin of $E_{\pi^{0}}^{*}$. Therefore the untagged samples of on-resonance data and $B \bar{B}$ simulation are fit, improving the statistics.

Improving the statistics is particularly useful for these fits, since they now include a background function to account for the random combinations of photon pairs. The default form of this background function is:

$$
f_{\gamma \gamma}(m)=\frac{a m^{b}}{\left(m^{2}+c\right)^{d}}
$$

where $a, b, c$ and $d$ are the free parameters of the fit. However, when the statistics available is very low, this default form is replaced with a simple first- or second-order polynomial.

Selected results from the second step of fits are tabulated in Table 4.4. For the fits to the $B \bar{B}$ MC samples, the agreement between the number of fitted $\pi^{0}$ mesons and the number of truth-matched candidates is still in good agreement, even in the presence of combinatorial background, as can be seen in Table 4.5. Note that the small over-estimation of the yield in the lower energy bins, at the $2-3 \%$ level, does 


\begin{tabular}{|c|c|c|c|c|}
\hline $\begin{array}{c}E_{\pi^{0}}^{*} \text { bin } \\
(\mathrm{GeV})\end{array}$ & \multicolumn{2}{|c|}{ BB MC sample } & \multicolumn{2}{c|}{ On-resonance data sample } \\
\cline { 2 - 5 } & $\mu_{1}\left(\mathrm{MeV} / c^{2}\right)$ & $\sigma_{1}\left(\mathrm{MeV} / c^{2}\right)$ & $\mu_{1}\left(\mathrm{MeV} / c^{2}\right)$ & $\sigma_{1}\left(\mathrm{MeV} / c^{2}\right)$ \\
\hline $1.0-1.2$ & $133.30 \pm 0.04$ & $5.77 \pm 0.04$ & $134.29 \pm 0.05$ & $6.40 \pm 0.05$ \\
$1.2-1.4$ & $133.58 \pm 0.03$ & $5.99 \pm 0.03$ & $134.36 \pm 0.03$ & $6.56 \pm 0.03$ \\
$1.4-1.6$ & $133.73 \pm 0.03$ & $6.38 \pm 0.03$ & $134.49 \pm 0.03$ & $6.85 \pm 0.03$ \\
$1.6-1.8$ & $133.88 \pm 0.03$ & $6.53 \pm 0.03$ & $134.62 \pm 0.03$ & $6.96 \pm 0.03$ \\
$1.8-2.0$ & $134.04 \pm 0.03$ & $6.76 \pm 0.03$ & $134.55 \pm 0.04$ & $7.21 \pm 0.04$ \\
$2.0-2.2$ & $134.49 \pm 0.04$ & $6.68 \pm 0.04$ & $134.76 \pm 0.04$ & $7.37 \pm 0.04$ \\
$2.2-2.4$ & $135.30 \pm 0.07$ & $6.69 \pm 0.07$ & $134.98 \pm 0.06$ & $7.26 \pm 0.06$ \\
$2.4-3.0$ & $135.86 \pm 0.17$ & $6.50 \pm 0.15$ & $135.09 \pm 0.05$ & $7.27 \pm 0.05$ \\
\hline
\end{tabular}

Table 4.4: Some selected results from the untagged $\pi^{0}$ mass fits: the peak position $\left(\mu_{1}\right)$ and the width $\left(\sigma_{1}\right)$ of the core Gaussian.

not affect the analysis, since $\pi^{0}$ mesons with these energies do not contribute photons in the $B \rightarrow X_{s} \gamma$ signal region.

\begin{tabular}{|c|r|r|c|}
\hline $\begin{array}{c}E_{\pi^{0}}^{*} \text { bin } \\
(\mathrm{GeV})\end{array}$ & \multicolumn{1}{|c|}{ Number of $\pi^{0}$ candidates } & \multicolumn{1}{c|}{$\begin{array}{c}\text { Ratio } \\
\text { (Fit/True })\end{array}$} \\
\hline $1.0-1.2$ & 69422 & \multicolumn{1}{|c|}{ Fitted } & \multicolumn{1}{c|}{$71644 \pm 469$} \\
$1.2-1.4$ & 143776 & $146433 \pm 556$ & $1.032 \pm 0.007$ \\
$1.4-1.6$ & 124083 & $126752 \pm 469$ & $1.022 \pm 0.0004$ \\
$1.6-1.8$ & 96205 & $96992 \pm 390$ & $1.007 \pm 0.004$ \\
$1.8-2.0$ & 72102 & $72261 \pm 328$ & $1.002 \pm 0.005$ \\
$2.0-2.2$ & 46232 & $45962 \pm 251$ & $0.994 \pm 0.005$ \\
$2.2-2.4$ & 13961 & $13813 \pm 137$ & $0.989 \pm 0.010$ \\
$2.4-3.0$ & 2985 & $3009 \pm 70$ & $1.008 \pm 0.023$ \\
\hline
\end{tabular}

Table 4.5: Validation of $\pi^{0}$ mass fits for untagged $B \bar{B}$ Monte Carlo events. The right-hand column gives the ratio of fitted candidates to the true number (Table 4.3). These results are for the full fits, including background from random combinations of photon pairs.

Finally, fits are performed on the tagged samples with only the parameters of the background function and the $A_{g}$ parameter free; the other parameters of $f_{\pi}^{0}(m)$ are set to the values determined in the untagged fits. (For the off-resonance data, the parameters from the corresponding untagged on-resonance fits are used.) Figures 4.2 
to 4.4 show these final fits (plotted on log-scale) for all energy bins for $B \bar{B} \mathrm{MC}$, onresonance and off-resonance data. The yields from fits are obtained by integrating $f_{\pi}^{0}(m)$

\subsubsection{Yields from $\eta$ Mass Distributions}

The procedure to extract the $\eta$ energy spectrum is very similar to the method used for $\pi^{0}$ mesons. Fits to the mass distributions are performed in five bins of $\eta$ c.m. energies: $1.0-1.5 \mathrm{GeV}, 1.5-1.7 \mathrm{GeV}, 1.7-1.9 \mathrm{GeV}, 1.9-2.2 \mathrm{GeV}$ and $2.2-2.6 \mathrm{GeV}$. For true $\eta$ mesons, the mass distribution is described by a two-sided CB function:

$$
f_{\eta}(m)= \begin{cases}N_{L}\left[\frac{p_{L} \sigma / \lambda_{L}}{\left(m_{L}-m\right)+p_{L} \sigma / \lambda_{L}}\right]^{p_{L}} & \text { for } m<m_{L} \\ A_{g} G(m, \mu, \sigma) & \text { for } m_{L}<m<m_{R} \\ N_{R}\left[\frac{p_{R} \sigma / \lambda_{R}}{\left(m-m_{R}\right)+p_{R} \sigma / \lambda_{R}}\right]^{p_{R}} & \text { for } m>m_{R}\end{cases}
$$

where $m_{L} \equiv \mu-\lambda_{L} \sigma$ and $m_{R} \equiv \mu+\lambda_{R} \sigma$ are the mass thresholds. The definitions of the various parameters are completely analogous to the $\pi^{0}$ case (Equation 4.11). For the combinatoric background, simple polynomials of first to third order are used, depending on the size of the data samples.

The procedure of successive fits is also applied for the $\eta$ spectra. For the truthmatched $B \bar{B}$ MC fits, all parameters of $f_{\eta}$ are left free. For the untagged fits on $B \bar{B}$ MC and on-resonance data, the $p$ and $\lambda$ parameters of the tails are fixed to their Monte Carlo values. In the final fits on the tagged samples, all parameters of $f_{\eta}(m)$ except $A_{g}$ and the parameters of the background polynomial function are fixed to their untagged values (Table 4.6). The yield in each bin of $E_{\eta}^{*}$, is obtained by integrating $f_{\eta}(m)$.

The performance of the $\eta$ mass fits is quantified in Table 4.7, where the yield from the fits are compared to the number of truth-matched candidates for the $B \bar{B} \mathrm{MC}$ samples. Here the fit overestimate the number of true candidates by a few percent in the lowest energy bins. The $\eta$ mesons in these bins do not contribute photons with energies in the $B \rightarrow X_{s} \gamma$ signal region, so these deviations are only relevant for $B \bar{B}$ control region checks. 

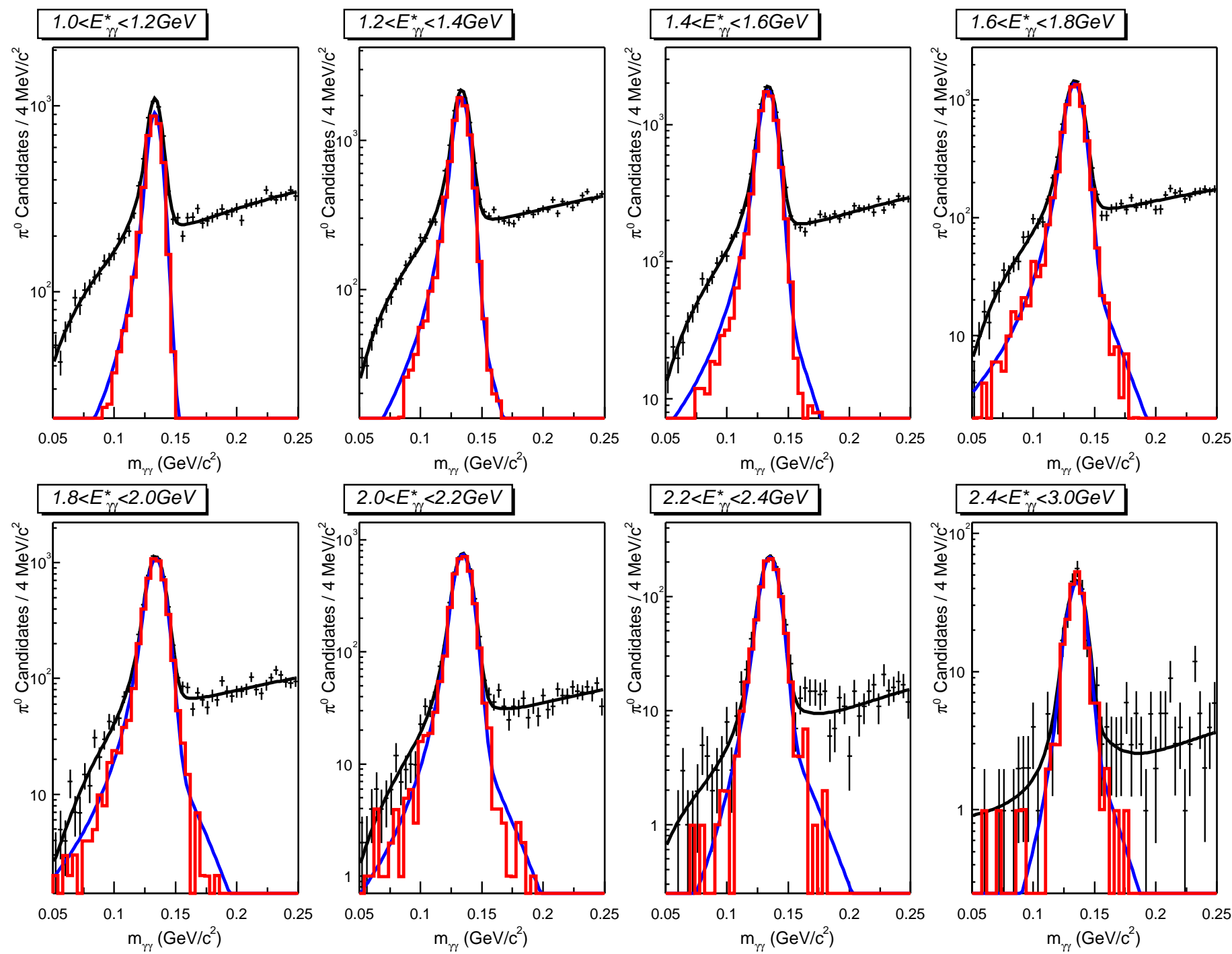



Figure 4.2: Fits to $\gamma \gamma$ mass for tagged $B \bar{B}$ Monte Carlo events in eight bins of $E_{\pi^{0}}^{*}$. The red histogram shows the truth-matched candidates. The $\pi^{0}$-signal part of the total fit is also shown as the lower smooth curve in blue. Statistical errors are shown on the data points. 

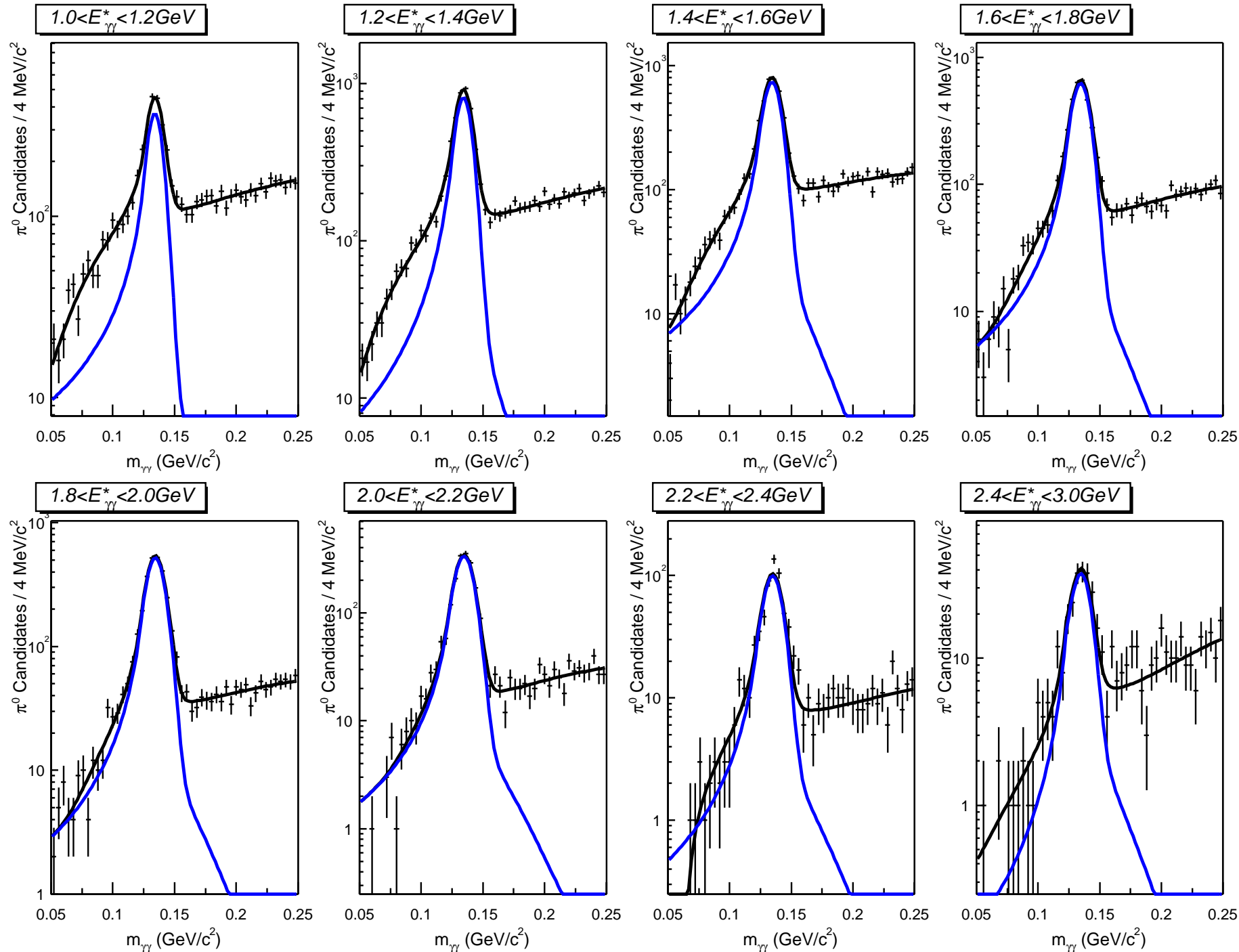

Figure 4.3: Fits to $\gamma \gamma$ mass for the tagged on-resonance data events in eight bins of $E_{\pi^{0}}^{*}$. The $\pi^{0}$-signal part of the total fit is shown as the lower smooth curve in blue. 

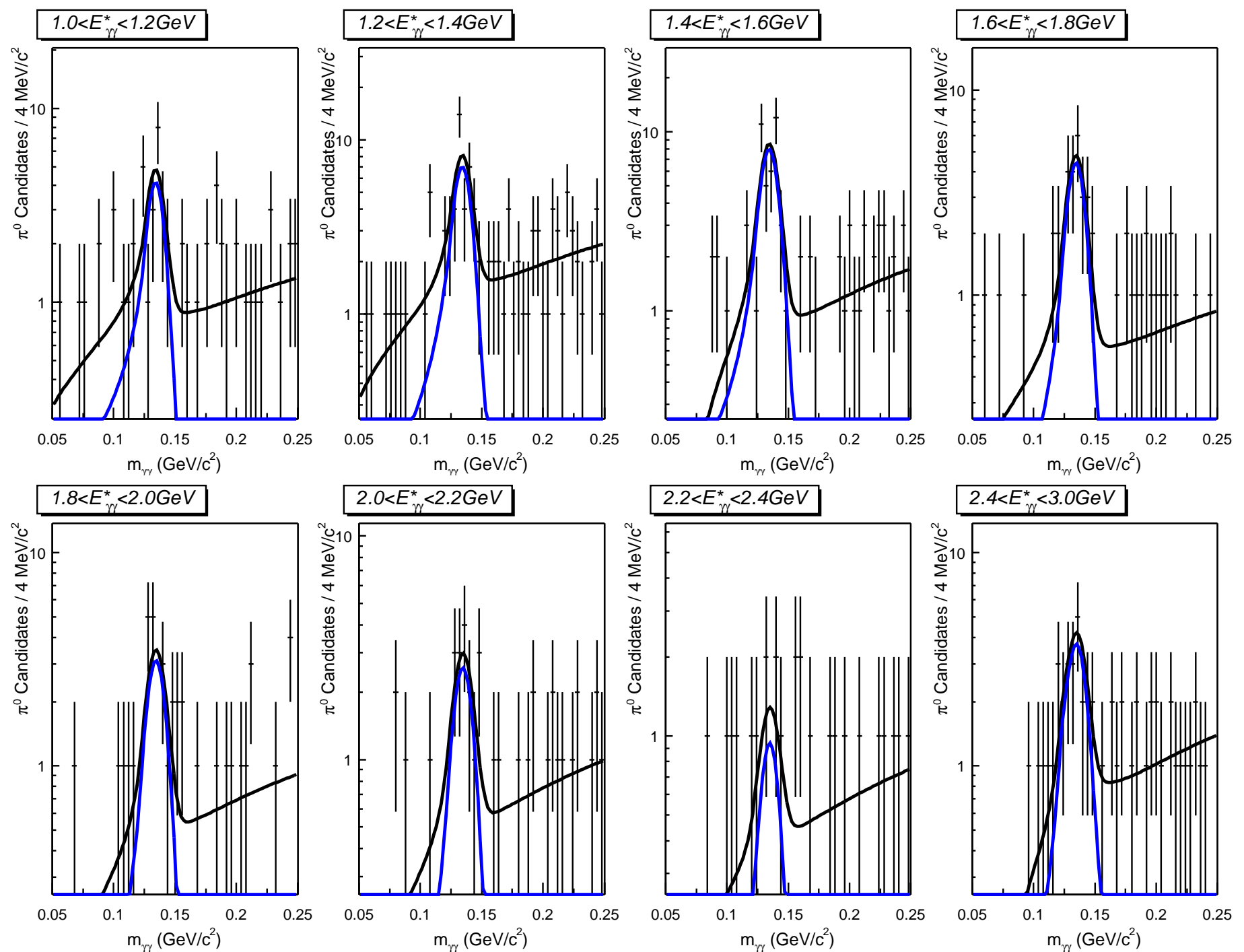

Figure 4.4: Fits to $\gamma \gamma$ mass for the tagged off-resonance data events in eight bins of $E_{\pi^{0}}^{*}$. The $\pi^{0}$-signal part of the total fit is shown as the lower smooth curve in blue. 


\begin{tabular}{|c|c|c|c|c|}
\hline \multirow{2}{*}{$\begin{array}{c}E_{\eta}^{*} \text { bin } \\
(\mathrm{GeV})\end{array}$} & \multicolumn{2}{|c|}{ BB MC sample } & \multicolumn{2}{c|}{ On-resonance data sample } \\
\cline { 2 - 5 } & $\mu\left(\mathrm{MeV} / c^{2}\right)$ & $\sigma\left(\mathrm{MeV} / c^{2}\right)$ & $\mu\left(\mathrm{MeV} / c^{2}\right)$ & $\sigma\left(\mathrm{MeV} / c^{2}\right)$ \\
\hline $1.0-1.5$ & $543.9 \pm 0.3$ & $14.0 \pm 0.3$ & $546.6 \pm 0.3$ & $15.5 \pm 0.3$ \\
$1.5-1.7$ & $543.8 \pm 0.2$ & $13.2 \pm 0.2$ & $546.4 \pm 0.3$ & $16.5 \pm 0.3$ \\
$1.7-1.9$ & $543.8 \pm 0.2$ & $13.6 \pm 0.2$ & $546.3 \pm 0.3$ & $15.5 \pm 0.3$ \\
$1.9-2.2$ & $543.7 \pm 0.2$ & $13.5 \pm 0.2$ & $546.0 \pm 0.2$ & $15.8 \pm 0.2$ \\
$2.2-2.6$ & $545.0 \pm 0.3$ & $13.8 \pm 0.3$ & $545.3 \pm 0.2$ & $16.3 \pm 0.2$ \\
\hline
\end{tabular}

Table 4.6: Some selected results from the untagged $\eta$ mass fits: the peak position $(\mu)$ and the width $(\sigma)$ of the Gaussian portion.

\begin{tabular}{|c|r|r|c|}
\hline $\begin{array}{c}E_{\eta}^{*} \text { bin } \\
(\mathrm{GeV})\end{array}$ & \multicolumn{2}{|c|}{ Number of $\eta$ candidates } & \multirow{2}{*}{$\begin{array}{c}\text { Ratio } \\
\text { (Fit/True })\end{array}$} \\
\hline $1.0-1.5$ & 32493 & \multicolumn{1}{c|}{ Fitted } & \\
$1.5-1.7$ & 29591 & $30735 \pm 677$ & $1.079 \pm 0.021$ \\
$1.7-1.9$ & 26766 & $27399 \pm 340$ & $1.039 \pm 0.014$ \\
$1.9-2.2$ & 26013 & $26468 \pm 294$ & $1.017 \pm 0.013$ \\
$2.2-2.6$ & 8880 & $8985 \pm 165$ & $1.012 \pm 0.019$ \\
\hline
\end{tabular}

Table 4.7: Validation of $\eta$ mass fits for untagged $B \bar{B}$ Monte Carlo events. The right-hand column gives the ratio of fitted candidates to the true number obtained from truth-matching. These results are for the full fits, including background from random combinations of photon pairs. 


\subsubsection{Energy Spectra and Correction Factors}

Figure 4.5 illustrates the yields of $\pi^{0}$ and $\eta$ mesons in $B \bar{B}$ events from data and simulation. As described earlier, the data spectrum is obtained by subtracting the yields in the off-resonance data from those in the on-resonance data. One observes that in the $B \rightarrow X_{s} \gamma$ signal region (for $E_{\gamma}^{*}>2.0 \mathrm{GeV}$ ), the $\pi^{0}$ background is measured to be higher in the data than in the simulation by $5-10 \%$. In contrast, the simulation is overestimating the $\eta$ yield. The ratio of the data to simulation yields are the needed correction factors. These are listed in Tables 4.8 and 4.9 ,
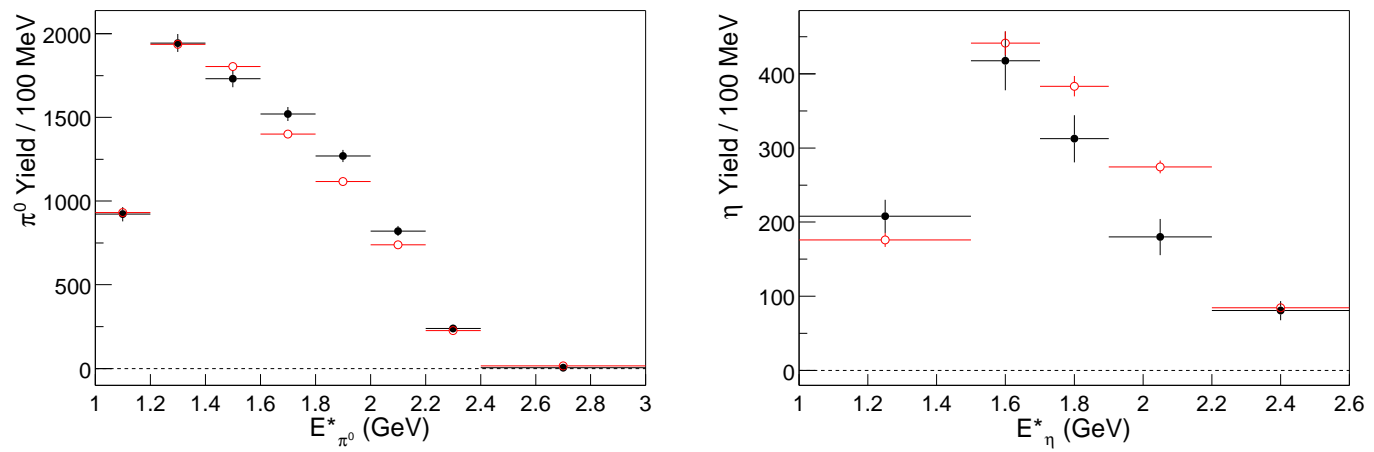

Figure 4.5: c.m.-energy spectra of $\pi^{0}$ (left) and $\eta$ (right) mesons for continuum subtracted data (solid circles in black) and for $B \bar{B}$ MC simulation (open circles in red). The errors are statistical only.

The systematic uncertainties have been calculated for these correction factors. For the $\pi^{0}$ mass fits, the apriori fixed values of $\mu_{2}$ and $\kappa$ are varied until the fit to the truth-matched mass distribution fails to converge. The other two parameters that are determined from fits to truth-matched $\pi^{0}$ candidates and later fixed in the remaining fits, $f_{1}$ and $\lambda$, are recomputed from new fits to data, in which all other parameters except the parameter in question and the total normalization are fixed. The change in the correction factors due to all these variations are taken as systematic errors. Moreover, statistical errors on the MC yields and the differences from unity in the fitted/true ratios in Table 4.5 are treated as additional sources of systematic uncertainties. The sum of all these systematic uncertainties in quadrature, tabulated in Tables 4.8 and 4.9 is smaller than the statistical uncertainty for each energy bin. 


\begin{tabular}{|c|l|}
\hline$E_{\pi^{0}}^{*}(\mathrm{GeV})$ & \multicolumn{1}{|c|}{ Data/MC Ratio } \\
\hline $1.0-1.2$ & $0.989 \pm 0.050 \pm 0.038$ \\
$1.2-1.4$ & $1.004 \pm 0.031 \pm 0.022$ \\
$1.4-1.6$ & $0.959 \pm 0.030 \pm 0.025$ \\
$1.6-1.8$ & $1.085 \pm 0.033_{-0.016}^{+0.011}$ \\
$1.8-2.0$ & $1.138 \pm 0.037_{-0.014}^{+0.013}$ \\
$2.0-2.2$ & $1.111 \pm 0.046_{0.016} \pm 0.016$ \\
$2.2-2.4$ & $1.062 \pm 0.092_{-0.019}^{+0.016}$ \\
$2.4-3.0$ & $0.35 \pm 0.57_{-0.19}^{+0.24}$ \\
\hline
\end{tabular}

Table 4.8: The $\pi^{0}$ correction factors, defined as the ratio of data to simulation yields, derived from the inclusive $\pi^{0}$ analysis. Statistical and systematic uncertainties are given.

\begin{tabular}{|c|c|}
\hline$E_{\eta}^{*}(\mathrm{GeV})$ & Data/MC Ratio \\
\hline $1.0-1.5$ & $1.146 \pm 0.141_{-0.103}^{+0.099}$ \\
$1.5-1.7$ & $0.945 \pm 0.096_{-0.044}^{+0.043}$ \\
$1.7-1.9$ & $0.816 \pm 0.088_{-0.029}^{+0.026}$ \\
$1.9-2.2$ & $0.656 \pm 0.091_{-0.023}^{+0.019}$ \\
$2.2-2.6$ & $0.96 \pm 0.16^{ \pm 0.05}$ \\
\hline
\end{tabular}

Table 4.9: The $\eta$ correction factors, defined as the ratio of data to simulation yields, derived from the inclusive $\eta$ analysis. Statistical and systematic uncertainties are given. 
The same procedure is repeated for determining the systematic errors on the $\eta$ correction factors. $p_{i}$ and $\lambda_{i}$ parameters, which are determined from the fits to the truth-matched $\eta$ candidates, are recomputed from new fits to data, in which only the parameter in question and the total normalization are allowed to vary. The effect of using these recomputed values is propogated to determine the errors. Statistical errors due to finite size of the MC samples and the deviations from unity in the fit/true ratios from Table 4.7 are added in quadrature.

It must be noted that the large uncertainties in the highest energy bins are not important. There are very few $\pi^{0}$ or $\eta$ mesons from those bins that contribute to the backgrounds in the $B \rightarrow X_{s} \gamma$ analysis.

\subsection{Backgrounds from Decays of Other Light Mesons}

Beyond $\pi^{0}$ and $\eta$, the next largest sources of real photons in the Monte Carlo $B \bar{B}$ background are $\omega$ and $\eta^{\prime}$ mesons (Table 4.1). Because their contributions are relatively small, corrections are derived only for their production rates, and possible small discrepancies that might arise from the effects of the analysis selection criteria can be ignored.

The inclusive $\omega$ spectrum in bins of $\omega$ momentum $\left(p_{\omega}^{*}\right)$ in the c.m. frame has been measured in an earlier BABAR analysis, based on a $20 \mathrm{fb}^{-1}$ subsample of the data used in this analysis [69. Table 4.10 lists the ratio of the yields from the measured spectrum to those from the generator-level spectrum in a small sample from $B \bar{B}$ simulation. Since the results in all four bins are consistent with each other, a common correction factor of $0.83 \pm 0.15$ is chosen to correct to the yield of $\omega$ candidates in the $B \bar{B}$ simulation.

Backgrounds from $\eta^{\prime}$ mesons not already accounted for by the $\pi^{0}(\eta)$ correction factors arise from $\rho^{0} \gamma, \pi^{+} \pi^{-} \gamma, \omega \gamma$ and $\gamma \gamma$ decay modes. The $\eta^{\prime}$ inclusive yield in $\Upsilon(4 S)$ events have also been measured by another earlier BABAR analysis, based on a method similar to that used for $\omega$ backgrounds in the same $20 \mathrm{fb}^{-1}$ subsample of the data [70]. By comparing the measured spectrum with the generator-level yields from the $B \bar{B}$ simulation correction factors are derived in two bins of $\eta^{\prime}$ reduced momentum, 


\begin{tabular}{|c|c|}
\hline$p_{\omega}^{*}(\mathrm{GeV} / c)$ & Data/MC Ratio \\
\hline $1.50-1.75$ & $0.84 \pm 0.14$ \\
$1.75-2.00$ & $0.81 \pm 0.15$ \\
$2.00-2.25$ & $0.83 \pm 0.28$ \\
$2.25-3.00$ & $0.49 \pm 0.75$ \\
\hline
\end{tabular}

Table 4.10: The ratio of the yields from the inclusive $\omega$ spectrum in data, as measured by an earlier BABAR analysis [69, to the spectrum predicted by the generator-level yields in $B \bar{B}$ MC simulation.

$x_{\eta}^{\prime} \equiv p_{\eta^{\prime}}^{*} / \sqrt{E_{\text {beam }}^{*}-m_{\eta^{\prime}}^{2}}: 0.49 \pm 0.15$ for $0.10<x_{\eta}^{\prime}<0.39$, and $1.77 \pm 0.58$ for $0.39<x_{\eta}^{\prime}<0.52$. The rationale for this choice of momentum intervals is that the lower region is dominated by $b \rightarrow c \rightarrow \eta^{\prime}$ decays, while the higher region is dominated by direct $B$ decays. Only the upper bin contributes to the signal region for this $B \rightarrow X_{s} \gamma$ analysis, but the lower bin can contribute to the lower- $E_{\gamma}^{*}$ control region.

\subsection{Background from Electrons}

The $B \bar{B}$ simulation has $9.1 \%$ of photon candidates truth matched to electrons and positrons in the control region of $1.5<E_{\gamma}^{*}<1.9 \mathrm{GeV}$. Therefore behind the photons from $\pi^{0}$ and $\eta$ decays, the showers from $e^{ \pm} \mathrm{S}$ constitute the third largest source of backgrounds in the control region. This statement is correct in the signal region of $2.0<E_{\gamma}^{*}<2.7 \mathrm{GeV}$ as well, after corrections described in the next section (Section (4.5) are applied to the antineutrons.

The Monte Carlo matching associates a reconstructed photon candidate to a true electron or positron in two cases, which can be considered as two distinct types of $e^{ \pm}$ backgrounds:

- Bremsstrahlung photons radiated from $e^{ \pm}$as they traverse the detector (particularly the beam pipe and the SVT support tube), are truth matched to the parent $e^{ \pm}$, since the particles created at the GEANT4 step of the simulation do not have true equivalents at the generator step. 
- A shower from the interaction of the $e^{ \pm}$with the calorimeter can satisfy the good photon criteria, if the $e^{ \pm}$track fails to be reconstructed or to be associated with its shower. Truth matching readily associates those showers to their true electrons or positrons.

The simulation of the material distribution in the detector has been verified by earlier BABAR measurements using radiative Bhabha events [73]. The number of radiation lengths in the simulation has been found to agree with its value in the data to better than $3 \%$. Therefore, the amount of $e^{ \pm}$backgrounds from Bremsstrahlung is not significantly different in data and in simulation, and the expected amount from the simulation can be used without any corrections.

However, the same cannot be easily argued for the second type of $e^{ \pm}$backgrounds. Although the BABAR simulation is good to $~ 99 \%$ in predicting the tracking efficiency of good tracks, the uncertainty on the earlier efficiency measurements is comparable to the size of the inefficiency [52. Similarly, the expectations for the inefficiency in track-shower association have been estimated with sizeable uncertainties that leave room for significant discrepancies between simulation and data [71.

The remainder of this section describes a precision-measurement of these inefficiencies and the effects of data-simulation discrepancies: Section 4.4.1 describes the measurement performed in Bhabha events, Section 4.4 .2 discusses the increase in the inefficiencies due to the high-multiplicity environment of multihadronic events, and Section 4.4.3 summarizes the corrections to the $B \bar{B}$ background predictions as a result of the observed differences between the simulation and the data.

\subsubsection{Electron Inefficiency in Bhabha Events}

The combined effect of the tracking and track-shower-association inefficiencies are initially measured using Bhabha events. The measurement sample consist of events passed by the Level 3 trigger for calibration purposes and prescaled to have a roughly flat distribution in track polar angle [72]. The trigger requires only one of the Bhabha tracks to be present, so the sample is suitable for the inefficiency measurement.

However, before proceeding, the sample needs to be tuned to the $B \rightarrow X_{s} \gamma$ 
analysis. At least two showers with $E>2.0 \mathrm{GeV}$ that satisfy the photon quality and shower shape and isolation criteria (Sections 3.3.1 and 3.3.2) are required. Among those, the two with the highest energy are assumed to be from the $e^{+} e^{-}$pair and their c.m.three-momenta at the IP are computed. The computation takes into account the effect of the magnetic field, the c.m.three-vectors at the IP are computed for two hypotheses: that the more (less) energetic shower is from an electron and the less (more) energetic shower is from a positron. The hypothesis with the larger acollinearity at the IP is then rejected, since the tracks from Bhabha events should be back-to-back in in the c.m. frame. To reject $e^{+} e^{-} \rightarrow e^{+} e^{-} e^{+} e^{-}$and $e^{+} e^{-} \rightarrow \gamma \gamma$ events, two criteria studied in earlier BABAR analyses are used [74]: the invariant mass of the $e^{+} e^{-}$candidate should be greater than $9 \mathrm{GeV} / c^{2}$ and the c.m. acollinearity at the IP should be smaller than 21.6 mrad.

The combined tracking and track-shower-association inefficiency is computed as $N_{\text {unassoc }}^{\text {non-Brem }} / N_{\text {assoc }}$, where $N_{\text {assoc }}$ is the total number of showers for which an associated track is present and $N_{\text {unassoc }}^{\text {non-Brem }}$ is the total number of showers to which no track is associated and for which no Bremsstrahlung evidence is present in the SVT or the DCH. (Hard Bremsstrahlung in the SVT support tube leaves high-momentum SVTonly tracks or low-momentum DCH-only tracks.) It is important to note that this computation gives essentially unbiased results, since the L3 tracking efficiency is very close to the tracking efficiency of the off-line reconstruction.

The inefficiency shows a dependence on the polar angle of the track. To account for the fact that the polar angle distribution of $e^{ \pm} \mathrm{s}$ from semileptonic decays are forward peaked due to the Lorentz boost of the $\Upsilon(4 S)$, the final inefficiency figure is computed by taking a weighted average polar angle bins. These weighted inefficiencies are listed in table 4.11, for three years of data taking. The inefficiency is larger in data than in the simulation.

\subsubsection{Inefficiency due to Multihadronic Environment}

Approximately $95 \%$ of $e^{ \pm}$backgrounds originate from semileptonic $B$ decays, the remainder are from the decay of $D$ mesons. The two-prong topology of a Bhabha 


\begin{tabular}{|l|c|c|}
\hline \multirow{2}{*}{ Year $($ DCH HV) } & \multicolumn{2}{|c|}{ Inefficiency $(\%)$} \\
\cline { 2 - 3 } $2000(1900 \mathrm{~V}$ and $1960 \mathrm{~V})$ & $0.190 \pm 0.003$ & $0.046 \pm 0.008$ \\
$2001(1930 \mathrm{~V})$ & $0.113 \pm 0.003$ & $0.048 \pm 0.008$ \\
$2002(1930 \mathrm{~V})$ & $0.102 \pm 0.004$ & $0.015 \pm 0.007$ \\
\hline
\end{tabular}

Table 4.11: The measurement of the combined tracking and track-showerassociation inefficiency by year in data and Monte Carlo simulation. The errors are statistical.

event is very different from the environment of a $B \bar{B}$ event with a semileptonic decay, where the mean charged track multiplicity is 9.5. The high multiplicity environment degrades the performance of the tracking algorithms. To observe the extent of this degradation, the SVT, DCH and EMC signals of properly reconstructed Bhabha tracks are embedded into random multihadronic events, and the reconstruction algorithms are rerun as outlined below:

1. Bhabha events where tracks are associated to both showers are selected in data and MC samples, which satisfy the requirements described in the previous section. The digitized SVT, DCH and EMC signals that constitute the reconstructed track and the shower for the electron and the positron are recorded.

2. A sample of multihadronic events with isotropic topology is selected from onresonance data and $B \bar{B} \mathrm{MC}$ simulation by requiring at least three tracks and $R_{2}^{*}<0.45$. Furthermore, events with high-momentum tracks $\left(P^{*}>2.5 \mathrm{GeV} / c\right)$ and high-energy showers $\left(E^{*}>3.5 \mathrm{GeV}\right)$ are rejected.

3. Embedding. The recorded signals from a Bhabha track are mixed with the signals of a multihadronic event. All the reconstruction algorithms are rerun.

4. The newly reconstructed event is searched for a shower that satisfies the photon selection criteria listed in the previous section and contains at least $50 \%$ of the EMC hits of the embedded $e^{ \pm}$. If such a shower is identified and no track is associated with it, the reconstruction is flagged as inefficient. 
The last two steps are repeated for each multihadronic event in the sample; typically the sample size is around 2000 events. This is considered one embedding experiment. Close to 1200 experiments are performed, one for each Bhabha track selected in the first step, enough to reach a statistical precision comparable to that of the basic inefficiency measurement performed in Bhabha events. The experiments are evenly distributed among various running conditions representative of the three years of data taking.

The resulting inefficiency has a strong dependence on the charged track multiplicity of the multihadron event. Therefore, in the computation of the inefficiency for each experiment, events are reweighted according to their multiplicity such that the multiplicity distribution matches that of simulated $B \bar{B}$ events with a semileptonic decay. The multiplicity-weighted inefficiencies of all experiments in a given month are averaged. Finally, to adjust for the difference in polar angular distributions between embedded Bhabha tracks and the $e^{ \pm} \mathrm{S}$ from semileptonic decays, events are weighted according to the semileptonic angular distribution in truth-matched simulation.

\begin{tabular}{|c|c||c|c|c||c|c|c|}
\hline Month & DCH & \multicolumn{3}{|c||}{ Data } & \multicolumn{3}{c|}{ Simulation } \\
\cline { 3 - 8 } & $\mathrm{HV}(\mathrm{V})$ & $N_{\exp }$ & $N_{\text {eff }}$ & Inefficiency (\%) & $N_{\exp }$ & $N_{\text {eff }}$ & Inefficiency (\%) \\
\hline Mar '00 & 1900 & 134 & 1235 & $0.121 \pm 0.009$ & 116 & 1027 & $0.145 \pm 0.011$ \\
Jun '00 & 1900 & 143 & 1423 & $0.135 \pm 0.008$ & 133 & 1074 & $0.120 \pm 0.009$ \\
\hline Aug '00 & 1960 & 147 & 1352 & $0.103 \pm 0.007$ & 123 & 1075 & $0.096 \pm 0.009$ \\
Oct '00 & 1960 & 124 & 1132 & $0.103 \pm 0.009$ & 128 & 1057 & $0.200 \pm 0.012$ \\
\hline Mar '01 & 1930 & 147 & 1452 & $0.527 \pm 0.016$ & 114 & 1033 & $0.090 \pm 0.008$ \\
Jun '01 & 1930 & 186 & 1327 & $0.113 \pm 0.007$ & 191 & 1061 & $0.182 \pm 0.009$ \\
Oct '01 & 1930 & 125 & 1268 & $0.106 \pm 0.008$ & 129 & 1038 & $0.160 \pm 0.011$ \\
\hline Mar '02 & 1930 & 109 & 1418 & $0.094 \pm 0.008$ & 108 & 1048 & $0.121 \pm 0.010$ \\
May '02 & 1930 & 74 & 1291 & $0.076 \pm 0.009$ & 118 & 1037 & $0.114 \pm 0.010$ \\
\hline
\end{tabular}

Table 4.12: The multiplicity and polar-angle weighted embedding inefficiencies measured in data and simulation for different running periods. $N_{\exp }$ is the number of experiments. $N_{\text {eff }}$ is the average effective number of multihadronic events per experiment after reweighting. The error on the inefficiency is statistical.

Table 4.12 summarizes the results of all the embedding experiments in data and simulation. In general the agreement is quite good between data and simulation, 
and among the different periods. There are three exceptions to this where one experiment biases the average to a large inefficiency: October 2000 simulation, June 2001 simulation and March 2001 data. These outlying experiments have inefficiencies greater than 1\%. Studies of the corresponding Bhabha tracks indicate no obvious anomalies that would lead to them failing the tracking or track-shower association so frequently. Moreover, the presence of the outliers in both data and MC samples lead to the conclusion that they represent rare but real effects, and thus they are not discarded from the final inefficiency computations. Unfortunately, the statistical errors on the inefficiencies can not adequately represent these outlying experiments, therefore a systematic uncertainty is assigned, which is discussed in the following section.

\subsubsection{Corrections to Electrons in $B \bar{B}$ Simulation}

\begin{tabular}{|c|c||c||c|}
\hline \multicolumn{2}{|c||}{ Period } & Data combined & MC combined \\
Year & DCH voltage (V) & inefficiency (\%) & inefficiency (\%) \\
\hline 2000 & 1900 & $0.318 \pm 0.007$ & $0.178 \pm 0.010$ \\
2000 & 1960 & $0.293 \pm 0.006$ & $0.181 \pm 0.010$ \\
2001 & 1930 & $0.365 \pm 0.007$ & $0.193 \pm 0.009$ \\
2002 & 1930 & $0.189 \pm 0.007$ & $0.133 \pm 0.010$ \\
\hline \hline \multicolumn{2}{|l||}{ Luminosity weighted average } & $0.296 \pm 0.004$ & $0.172 \pm 0.006$ \\
\hline
\end{tabular}

Table 4.13: The $e^{ \pm}$tracking inefficiency for data and for simulation by running period. The luminosity weighted average is also given. The errors are statistical only.

Table 4.13 lists the combined inefficiencies from the basic Bhabha measurement and the additional inefficiency from the degradation in the high-multiplicity environment as observed by the embedding study. The observed difference between the data and simulation, $\Delta \epsilon_{e^{ \pm}}=0.124 \pm 0.007 \%$, is used to calculate a correction to the number of high-energy photon candidates truth-matched to $e^{ \pm}$in the $B \bar{B}$ simulation. 
The number of additional showers in each $E_{\gamma}^{*}$ bin, $\Delta N_{\text {shower }}\left(E_{\gamma}^{*}\right)$, is given by:

$$
\Delta N_{\text {shower }}\left(E_{\gamma}^{*}\right)=N_{\mathrm{SL}}\left(p_{e^{ \pm}}^{*}\right) \times \epsilon_{\text {sel }}\left(E_{\gamma}^{*}\right) \times \Delta \epsilon_{e^{ \pm}}
$$

Here $N_{\mathrm{SL}}\left(p_{e^{ \pm}}^{*}\right)$ is the expected number of $B \rightarrow X e \nu$ decays with a lepton of c.m.momentum, $p_{e^{ \pm}}^{*}$, within the limits of the $E_{\gamma}^{*}$ bin and of polar angle $-0.74<\cos \theta_{e^{ \pm}}<$ 0.93. Obtained from simulation and scaled to the on-resonance integrated luminosity, $N_{\mathrm{SL}}\left(p_{e^{ \pm}}^{*}\right)$ is not given as a function of $E_{\gamma}^{*}$, but this is not a significant source of bias, since $p_{e^{ \pm}}^{*} \approx E_{\text {shower }}^{*}$.

$\epsilon_{\text {sel }}\left(E_{\gamma}^{*}\right)$ is the efficiency of the event selection criteria (of chapter 3) on $B \bar{B}$ events where the shower of an $e^{ \pm}$of polar angle $-0.74<\cos \theta_{e^{ \pm}}<0.93$ becomes a background due to failure of tracking or track-shower association. Since the truthmatching does not distinguish Bremsstrahlung and inefficiency related $e^{ \pm}$backgrounds, this quantity is determined from all $e^{ \pm}$background events in the $B \bar{B}$ simulation that satisfy $E_{\text {shower }} / p_{e^{ \pm}}^{\text {true }}>0.85$. This requirement significantly suppresses the fraction of Bremsstrahlung related $e^{ \pm}$backgrounds.

The overall corrections to the $B \bar{B}$ simulation are summarized in Table 4.14, The computed number of additional $e^{ \pm}$showers is given also as a fraction of the number of $e^{ \pm}$background events in the $B \bar{B}$ simulation. In the light of various earlier measurements and validation checks, $50 \%$ of the correction in each bin is conservatively assigned as systematic uncertainty to account for various assumptions made in this study: that the inefficiency is the same for leptons from semileptonic events even though they are of lower momentum than the Bhabha tracks used; that a $p_{e^{ \pm}}^{*}$ bin corresponds to the same bin of $E_{\gamma}^{*}$; that the semileptonic multiplicity is well modeled in the simulation; that the selection efficiencies, $\epsilon_{\text {sel }}\left(E_{\gamma}^{*}\right)$, are accurate and not significantly different in simulation and data; that it is valid to include three outlying embedding experiments discussed in the earlier section in the computation. (If they are dismissed, the corrections are reduced by 35\%.)

One validation check worth mentioning is performed by using the measured inefficiency in the simulation, $0.172 \pm 0.006$, and the selection criteria efficiencies from Table 4.14, to compute the number of ineffiency-caused background events in the 


\begin{tabular}{|c|c|c|c|c|}
\hline $\begin{array}{c}E_{\gamma}^{*} \\
(\mathrm{GeV})\end{array}$ & \multicolumn{1}{c|}{$\begin{array}{c}N_{S L} \\
\left(\times 10^{3}\right)\end{array}$} & $\begin{array}{c}\epsilon_{\text {sel }} \\
(\%)\end{array}$ & $\Delta N_{\text {shower }}$ & $\begin{array}{c}\Delta N_{\text {shower }} / N_{e^{ \pm}-\mathrm{BG}} \\
(\%)\end{array}$ \\
\hline $1.6-1.7$ & $1279.4 \pm 17.7$ & $0.20 \pm 0.03$ & $3.2 \pm 0.5$ & $8.8 \pm 4.4$ \\
$1.7-1.8$ & $1199.8 \pm 15.9$ & $0.31 \pm 0.04$ & $4.7 \pm 0.6$ & $11.7 \pm 5.9$ \\
$1.8-1.9$ & $1024.6 \pm 15.9$ & $0.36 \pm 0.04$ & $4.6 \pm 0.6$ & $15.4 \pm 7.8$ \\
$1.9-2.0$ & $829.9 \pm 14.2$ & $0.48 \pm 0.06$ & $4.9 \pm 0.7$ & $22.7 \pm 11.5$ \\
$2.0-2.1$ & $520.2 \pm 10.6$ & $0.62 \pm 0.07$ & $4.0 \pm 0.5$ & $30.7 \pm 15.5$ \\
$2.1-2.2$ & $246.0 \pm 7.1$ & $0.51 \pm 0.19$ & $1.5 \pm 0.6$ & $39.0 \pm 19.9$ \\
$2.2-2.3$ & $83.2 \pm 3.5$ & $0.53 \pm 0.31$ & $0.6 \pm 0.3$ & $38.6 \pm 20.1$ \\
$2.3-3.5$ & $26.5 \pm 1.8$ & $0.57 \pm 0.23$ & $0.2 \pm 0.1$ & $13.5 \pm 7.1$ \\
\hline
\end{tabular}

Table 4.14: Bin-by-bin corrections to the number of $\mathrm{MC}$ background $e^{ \pm} \mathrm{S}$ due to the data-vs.-simulation differences in the tracking and track-showerassociation inefficiencies. Also shown are the number $N_{S L}$ of $B \rightarrow X e^{ \pm} \nu$ decays in $88.5 \times 10^{6} B \bar{B}$ events with $p_{e^{ \pm}}^{*}$ in the same interval as the $E_{\gamma}^{*}$ bin, and the selection efficiencies $\left(\epsilon_{\text {sel }}\right)$. The fractional correction to the number of background photon candidates from $e^{ \pm}$in the simulation, $N_{e^{ \pm}-\mathrm{BG}}$, is given in the rightmost column. The uncertainties are statistical only in all columns except the last, for which the systematic uncertainty is included.

$B \bar{B}$ simulation. This number should be smaller than the number of $e^{ \pm}$background events reported by the truth-matching, since the latter includes Bremsstrahlungrelated backgrounds as well. The ratio of the two is indeed smaller than unity and rises between 0.10 and 0.54 with increasing $E_{\gamma}^{*}$, consistent with fact that lower $E_{\gamma}^{*}$ bins should have a higher fraction of Bremsstrahlung photons, downfeeding from higher values of $p_{e^{ \pm}}^{*}$.

\subsection{Hadronic Component of the Background}

Most of the $B \bar{B}$ backgrounds are due to electromagnetic interactions in the calorimeter, which are modeled well in the simulation. However, as shown in Table 4.1 a significant background component is also expected from hadrons in the $B \bar{B}$ simulation, even after the shower shape requirements described in Section 3.3.2. The 
hadronic interactions are simulated with Gheisha [75] in the BABAR Monte Carlo production and large uncertainties are associated with them.

The Monte Carlo prediction for background hadrons is dominated by antineutrons. This is not surprising, since charged-hadron contributions are only due to inefficiencies in track reconstruction or track-shower matching and antibaryon annihilation provides an extra $0.94 \mathrm{GeV}$ beyond the energy of the particle, so antineutrons can much more easily populate the high- $E_{\gamma}^{*}$ region of interest.

Possible discrepancies in the simulated antineucleon background can be due to uncertainties in the production cross section, in the shower energy deposition, and in the simulation of the shower shape variables. Each of these are studied in the following sections.

\subsubsection{Antinucleon Production}

Although there are no measurements of the inclusive antineutron spectrum at $B A B A R$, it is possible to measure the spectra for antiprotons and assume that the corrections to the cross sections are applicable to both kinds of antinucleons, to the extent that isospin is conserved in $B$ decays. A $p / \bar{p}$ spectrum measurement has been performed in an earlier BABAR analysis on a small subset $\left(\sim 5 \mathrm{fb}^{-1}\right)$ of the data [76]. Comparison with $B \bar{B}$ MC simulation indicates that the $p / \bar{p}$ production is overestimated in the simulation (Figure 4.6) and that the antineutron yields in each bin of $E_{\gamma}^{*}$ should be reduced by $30-40 \%$.

However, before applying any such corrections, it is important to verify the assumption about the applicability of the $p / \bar{p}$ results to antineutrons. Significant differences to the production rate are expected in weak decays, e.g., the decay of $\bar{\Lambda}$ where branching fractions to $\bar{p} \pi^{+}$and $\bar{n} \pi^{0}$ final states are $64 \%$ and $36 \%$, respectively [4]. Therefore, a study of the antinucleon production in the $B \bar{B}$ background simulation is performed to compare the parentage, momentum distribution, and total production rates.

As shown in Table 4.15, there are three main sources of antinucleons in $B \bar{B}$ events: prompt production from $B$ mesons, strong decays of intermediate $\Delta$ resonances, and 


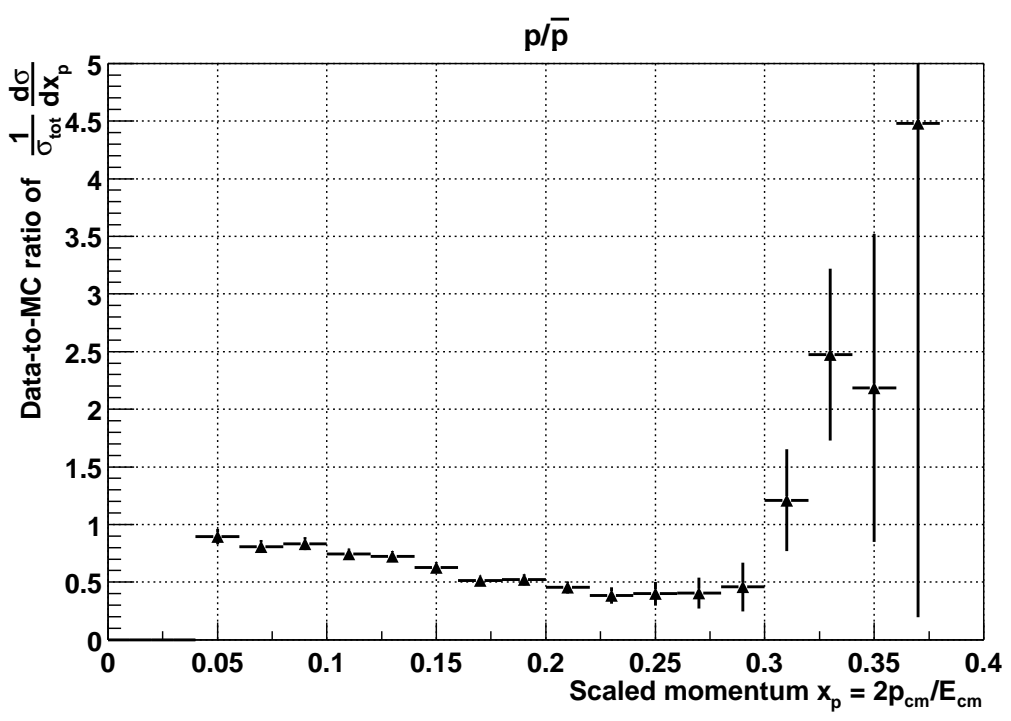

Figure 4.6: Ratio of the differential cross section for $p / \bar{p}$ production from the $\Upsilon(4 S)$ mesons in data to the corresponding cross section in the Monte Carlo event generator for $B \bar{B}$ simulation [76]. The horizontal scale is the scaled $p / \bar{p}$ momentum in the c.m.frame.

\begin{tabular}{|l|c|c|}
\hline Parent & $\bar{n}(\%)$ & $\bar{p}(\%)$ \\
\hline$B$ & $25.0 \pm 0.3$ & $21.8 \pm 0.2$ \\
$\bar{\Lambda}, \bar{\Sigma}^{ \pm}$and $\bar{\Lambda}_{c}$ & $38.5 \pm 0.3$ & $37.5 \pm 0.3$ \\
$\Delta$ & $34.4 \pm 0.3$ & $39.2 \pm 0.3$ \\
Charmonium & $2.1 \pm 0.1$ & $1.5 \pm 0.1$ \\
\hline
\end{tabular}

Table 4.15: The parentage of $\bar{n} \mathrm{~s}$ and $\bar{p} \mathrm{~s}$ in the $B \bar{B}$ background simulation. The fractions are the average of $B^{0} \bar{B}^{0}$ and $B^{+} B^{-}$simulations. 
weak decays of strange and charm antibaryons $\left(\bar{\Lambda}, \bar{\Lambda}_{c}\right.$, and $\left.\bar{\Sigma}^{ \pm}\right)$. There is also a small contribution from charmonium decays to $n \bar{n}$ and $p \bar{p}$ pairs. The parentage fractions are very similar for the two kinds of antinucleons, with the largest relative difference being $15 \%$ for the fraction of $\Delta$ parents.
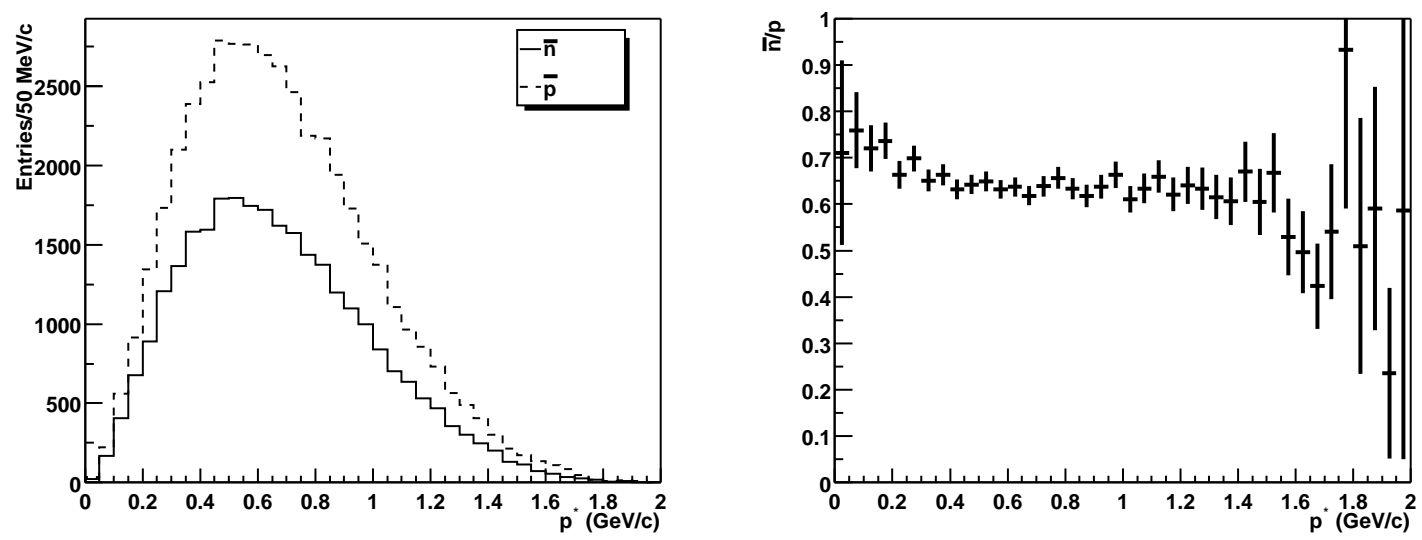

Figure 4.7: The c.m.momentum spectra of antinucleons (left) and their ratio to one another (right) from the $B \bar{B}$ background simulation. The dashed (solid) histogram is for antiprotons (antineutrons). The error bars indicate statistical uncertainties only.

The c.m.-momentum distributions in the $B \bar{B}$ background simulation are shown in Figure 4.7. The difference in the normalizations is dominated by the number of antineutrons produced in the $B^{+} B^{-}$simulation being only half the number of antiprotons. (The $N_{\bar{n}} / N_{\bar{p}}$ ratio in $B^{0} \bar{B}^{0}$ is 0.85 .) Qualitatively, this is not suprising: The most likely parton composition of a $B^{-}$decay where $W^{-}$decays to $\bar{u} d$ is $c d \overline{u u}$, so the creation of a single $d \bar{d}$ pair during hadronization is enough to produce an antiproton, whereas at least two pairs are needed for an antineutron.

However this difference in the absolute yields is not important. The ratio of the c.m.-momentum distributions is approximately constant at 0.65 over the interval $0.2<p_{\bar{p} / \bar{n}}^{*}<1.5 \mathrm{GeV} / c$, which contains over $90 \%$ of the particles. The similarity in the relative composition of the parentage and in the spectral shapes indicate that the use of corrections to $\bar{n}$ production derived from $\bar{p}$ measurements is valid, irrespective 
of the normalization difference.

\subsubsection{Studies of Antinucleon Energy Deposition}

The deficiencies of the GEANT4 implementation of Gheisha in the prediction of the interactions of charged and neutral hadrons with calorimeters have been raised in the literature [77. Although the annihilation of antinucleons at rest is reported to be reasonably well modeled, significant problems are present in the predictions of the energy deposits when the antinucleon carries momenta. Therefore it is imperative to investigate the energy deposition of antineutrons in the calorimeter and derive corrections as a function of their momenta.

Unfortunately, there is no clean data sample of antineutrons with which to do such an investigation. However, it has been reported that the discrepancies observed for protons and neutrons are essentially identical. This is certainly no surprise - Once a hadronic shower is initiated, its further development is not likely to depend on the identity of the initiating nucleon. Moreover, comparisons of antiproton interaction cross sections on protons and deuterons over a wide range of momenta [4] imply that antinucleon-nucleon cross sections are largely independent of the particular kind of particles involved. Therefore a sample of antiprotons can be used instead of antineutrons for the purposes of deriving corrections to hadronic interactions. Antiprotons that do not interact hadronicly loose energy by ionization, but calorimeter deposits from those will have too low energies to be of concern for the $B \rightarrow X_{s} \gamma$ analysis.

Clean samples of antiprotons from $\bar{A}$ decays are available in on-resonance data and in MC simulation $(q \bar{q}$ and $B \bar{B})$ samples, which cover the same antiproton kinematic regions [78. The quantity of interest is the deposited c.m.-energy, $E_{\gamma}^{*}$, as a function of the antiproton c.m.-momentum, $p_{\bar{p}}^{*}$. Figure 4.8 shows the $E_{\gamma}^{*}$ spectra normalized to $p_{\bar{p}}^{*}$, in bins of $p_{\bar{p}}^{*}$. For each bin, the data and simulation histograms have been normalized to have the same area, including the low- $E_{\gamma}^{*} / p_{\bar{p}}^{*}$ region which is not illustrated.

While the details of the comparison vary with $p_{\bar{p}}^{*}$, two general features are apparent: (i) for most of the bins, the simulation overestimates the fraction of the spectrum above the minimum-ionizing peak (consistent with what has been observed by other 


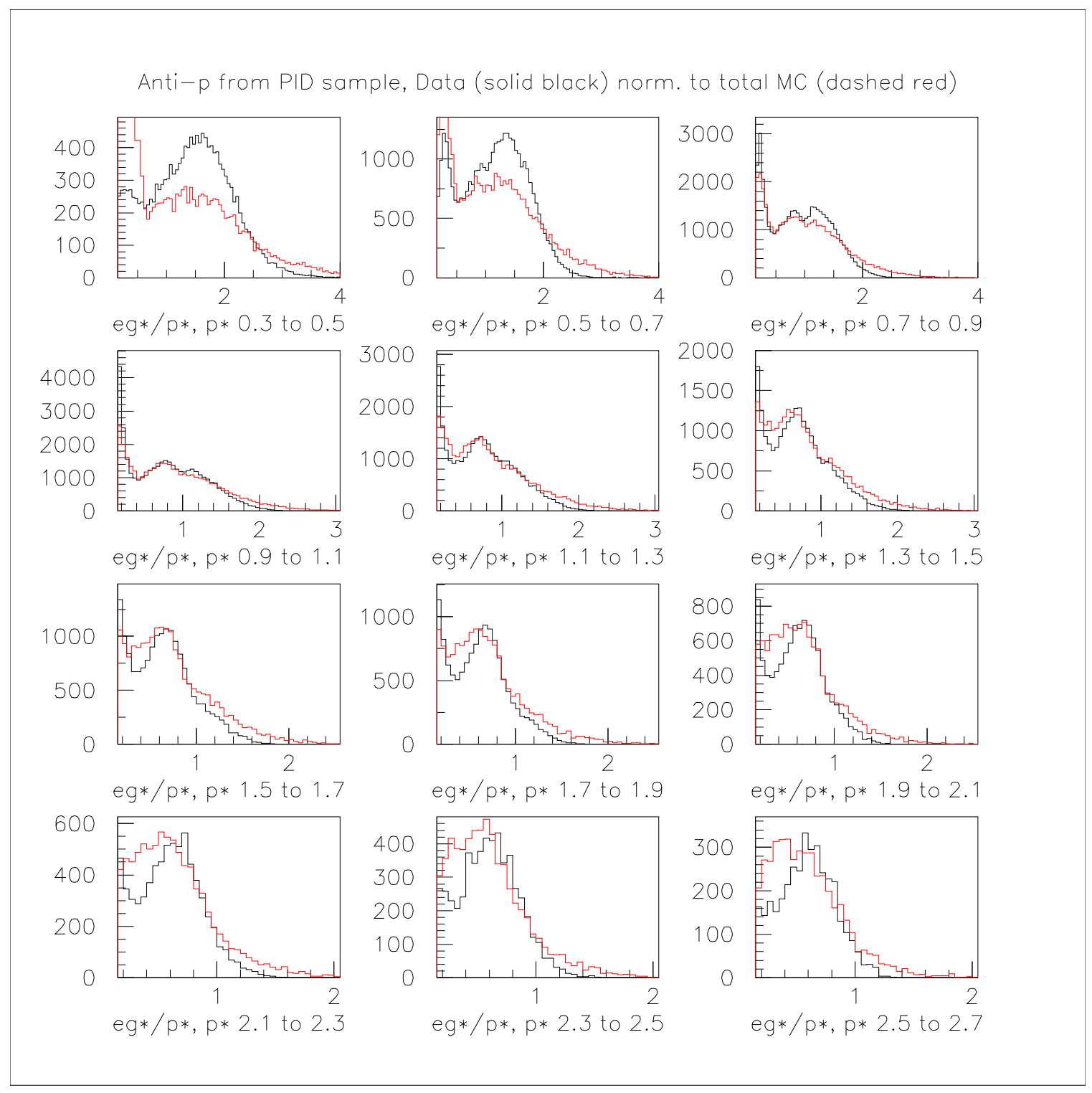

Figure 4.8: Comparison of calorimeter response to antiprotons in control samples from data (solid black) and simulation (dashed red). Plotted are spectra of the ratio of the deposited c.m.-energy, $E_{\gamma}^{*}$, to the antiproton c.m.momentum, $p_{\bar{p}}^{*}$, in bins of $p_{\bar{p}}^{*}$. The data histogram in each bin is normalized to have the same area as the simulation histogram, including the low-end minimum-ionizing peak, which is truncated for purposes of illustration. 
experiments [79]), and (ii) for all bins, the simulation substantially overestimates the high-end tail, to a level that is clearly unphysical; the simulation is predicting energy deposits larger than the maximum available (Figure 4.9).

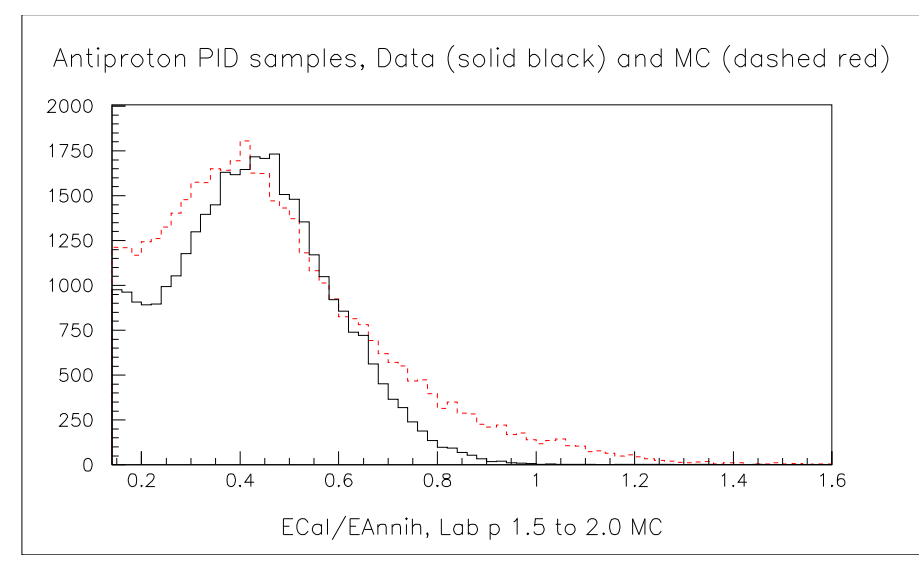

Figure 4.9: Distributions of the ratio of the EMC shower energy to the total available energy $\left(E_{\bar{p}}+m_{p} c^{2}\right)$ for antiproton control samples in data and simulation, plotted for an example antiproton momentum interval of 1.5$2.0 \mathrm{GeV} / c$. The data distribution (solid black) has been normalized to have the same integral as the MC distribution (dashed red) over the full range, including the low-end (minimum-ionizing) region which is not illustrated.

After a check that the simulation has indeed the same predictions for $E_{\gamma}^{*} / p_{\bar{p}}^{*}$ distributions for antineutrons and antiprotons, the differences in the spectra observed in Figure 4.8 can be tranformed into correction factors applicable to both antineutron and antiprotons. This is done as a function of both $E_{\gamma}^{*}$ and $p_{\bar{p}}^{*}$ and the resultant correction factors are listed (rounded to two significant figures) in Table 4.16. The values imply a substantial reduction of the predicted amount of hadronic backgrounds in the $B \bar{B}$ simulation. Fluctuations in the lower $p_{\bar{p}}^{*}$ bins are due to limited statistics.

\subsubsection{Studies of Shower Shape Variables}

As the last step of the corrections to the simulation of hadronic interactions, possible differences in second and lateral moment distributions should be studied. For a given value of $p_{\bar{p}}^{*}$, events with photon candidates of lower moments (the ones that satisfy 


\begin{tabular}{|c|c|c|c|c|c|c|c|c|c|}
\hline$E_{\gamma}^{*}$ & \multicolumn{10}{|c|}{$p_{\bar{p}}^{*}(\mathrm{GeV} / c)$ bin } \\
\cline { 2 - 10 }$(\mathrm{GeV})$ & 0.4 & 0.6 & 0.8 & 1.0 & 1.2 & 1.4 & 1.6 & 1.8 & 2.0 \\
\hline $1.6-1.7$ & 0.10 & 0.44 & 0.81 & 0.91 & 0.86 & 0.83 & 0.97 & 1.00 & 1.12 \\
$1.7-1.8$ & 0.07 & 0.31 & 0.63 & 0.89 & 0.79 & 0.75 & 0.84 & 0.98 & 1.08 \\
$1.8-1.9$ & 0.04 & 0.23 & 0.61 & 0.74 & 0.84 & 0.69 & 0.74 & 0.94 & 0.95 \\
$1.9-2.0$ & 0.04 & 0.13 & 0.43 & 0.73 & 0.68 & 0.71 & 0.72 & 0.96 & 1.02 \\
$2.0-2.1$ & 0.04 & 0.10 & 0.31 & 0.51 & 0.60 & 0.64 & 0.74 & 0.79 & 0.82 \\
$2.1-2.2$ & 0.06 & 0.09 & 0.27 & 0.44 & 0.60 & 0.64 & 0.76 & 0.75 & 0.99 \\
$2.2-2.3$ & 0.04 & 0.05 & 0.17 & 0.34 & 0.52 & 0.44 & 0.59 & 0.77 & 0.94 \\
$2.3-2.4$ & 0.04 & 0.03 & 0.06 & 0.32 & 0.43 & 0.40 & 0.43 & 0.64 & 0.60 \\
$2.4-2.5$ & 0.09 & 0.06 & 0.11 & 0.19 & 0.37 & 0.39 & 0.40 & 0.49 & 0.70 \\
$2.5-2.6$ & 0.06 & 0.04 & 0.05 & 0.14 & 0.25 & 0.26 & 0.43 & 0.46 & 0.44 \\
$2.6-2.7$ & 0.05 & 0.09 & 0.04 & 0.10 & 0.18 & 0.20 & 0.39 & 0.45 & 0.57 \\
$2.7-2.8$ & 0.10 & 0.02 & 0.06 & 0.06 & 0.22 & 0.12 & 0.26 & 0.40 & 0.48 \\
\hline
\end{tabular}

Table 4.16: Data/MC correction factors from antiproton PID control samples, in bins of the c.m.-energy deposited in the calorimeter, $E_{\gamma}^{*}$, vs. antiproton c.m.-momentum, $p_{\bar{p}}^{*}$. For the $p_{\bar{p}}^{*}$ bins, only the center point of the bin is given, labeling an interval within $\pm 100 \mathrm{MeV} / c$ of that point. The data and the simulation subsamples in each $p_{\bar{p}}^{*}$ bin have been normalized to have the same number of entries before the ratios in that bin are computed.

\begin{tabular}{|c|c|c|c|c|c|}
\hline & \multicolumn{5}{|c|}{$E_{\gamma}^{*}(\mathrm{GeV})$} \\
\cline { 2 - 6 } & 1.6 & 1.7 & 1.8 & 1.9 & 2.2 \\
& to & to & to & to & to \\
$p_{\bar{p}}^{*}(\mathrm{GeV} / c)$ & 1.7 & 1.8 & 1.9 & 2.2 & 2.7 \\
\hline $0.3-1.1$ & 0.27 & 0.18 & 0.24 & 0.16 & 0.24 \\
$1.1-1.7$ & 0.73 & 0.74 & 0.79 & 0.51 & 0.42 \\
\hline
\end{tabular}

Table 4.17: Data/MC correction factors for the effect of photon-selection second moment and LAT requirements, from antiproton PID control samples. These are shown in default bins of $E_{\gamma}^{*}$ vs. $p_{\bar{p}}^{*}$, where $E_{\gamma}^{*}$ is the deposited energy in the calorimeter boosted to the c.m. frame. 
the shower shape criteria) tend to have lower $E_{\gamma}^{*}$ than events failing the shower shape criteria, but the simulation disagrees with the data as to the size of this effect. To correct for this disagreement, the ratio of the efficiency of the shower shape criteria in data to that in the simulation can be used as correction factors, as given in Table 4.17. Due to inadequate statistics, larger bins are used. Since there are very few antibaryons in the $B \bar{B}$ simulation with $p_{\bar{p}}^{*}$ above $1.7 \mathrm{GeV} / c$, the still-inadequate statistics for the corrections in this region don't matter; the corrections are derived from below that momentum. The same is done for $E_{\gamma}^{*}$ above $2.7 \mathrm{GeV}$.

\subsubsection{Corrections to the Antinucleons in $B \bar{B}$ Simulation}

In order to compute the overall corrections to the antibaryon (primarily $\bar{n}$ but also $\bar{p}$ ) background, the corrections derived in the last three subsections are all combined. All three corrections are functions of the true antibaryon c.m. momentum, while the latter two are also functions of the apparent photon c.m.energy $\left(E_{\gamma}^{*}\right)$. The average weight over events in a given $E_{\gamma}^{*}$ bin is the correction factor for that bin; results are shown in Table 4.18. Conservative systematic uncertainties of $50 \%$ for the cross sections and $50 \%$ for the combined calorimeter response effects lead to an overall uncertainty of $71 \%$, which far outweighs any statistical uncertainties in the determination of the factors.

\subsection{Final-State Radiation from Light Quarks}

PHOTOS package is used to simulate QED radiative effects in the $B \bar{B}$ simulation for final states with electrons and muons. The photons with $B$-meson parentage given in Table 4.1 are due to the PHOTOS-generated final-state radiation (FSR) in semileptonic decays. However, FSR from light quarks during fragmentation is not simulated by the implementation of Jetset within EvtGen. To account for this not-simulated source of background photons, analytical estimates are made.

A calculation of the quark level FSR process $b \rightarrow c \bar{u} d+\gamma$ is given in [80]. Figure 4.10 shows the normalized spectrum for this process as a function of the photon 


\begin{tabular}{|c|c|}
\hline$E_{\gamma}^{*}(\mathrm{GeV})$ & $\bar{n}$ Correction \\
\hline $1.6-1.7$ & $0.13 \pm 0.09$ \\
$1.7-1.8$ & $0.10 \pm 0.07$ \\
$1.8-1.9$ & $0.11 \pm 0.08$ \\
$1.9-2.0$ & $0.06 \pm 0.04$ \\
$2.0-2.1$ & $0.06 \pm 0.04$ \\
$2.1-2.2$ & $0.05 \pm 0.03$ \\
$2.2-2.3$ & $0.03 \pm 0.02$ \\
$2.3-2.4$ & $0.04 \pm 0.03$ \\
$2.4-2.5$ & $0.03 \pm 0.02$ \\
$2.5-2.6$ & $0.04 \pm 0.03$ \\
$2.6-2.7$ & $0.02 \pm 0.02$ \\
$2.7-2.8$ & $0.02 \pm 0.01$ \\
\hline
\end{tabular}

Table 4.18: The combined corrections for the production rate and the calorimeter response of background antineutrons in the $B \bar{B}$ simulation, in $100 \mathrm{MeV}$ bins of $E_{\gamma}^{*}$.

energy in the $b$-quark rest frame, $E_{\gamma}^{b-\text { rest }}$, for $m_{b}=4.8 \mathrm{GeV} / c^{2}$. The normalization factor, $\Gamma_{0}$, is the tree-level contribution to the process $B \rightarrow X_{s} \gamma$ from the electromagnetic dipole operator, $\mathcal{O}_{7}$, in the effective Hamiltonian (Section 1.4). It is given by:

$$
\Gamma_{0}=\frac{G_{F}^{2}\left|V_{t b} V_{t s}^{*}\right|^{2} \alpha_{\mathrm{em}} C_{7}^{2}}{32 \pi^{4}} m_{b}^{5}=1.17 \times 10^{-16} \mathrm{GeV}
$$

The numerical value of $\Gamma_{0}$ is calculated by taking values of the Fermi constant, $G_{F}$, the CKM factors, $V_{t x}$, and the electromagnetic fine-structure constant, $\alpha_{\mathrm{em}}$, from the Particle Data Group [4] and the Wilson coefficient to be $C_{7}=-0.306$ [81]. The differential spectrum is scaled to a differential branching fraction using a factor $\frac{\Gamma_{0}<\tau_{B}>}{\hbar}$, where $<\tau_{B}>=1.6 \mathrm{ps}$ is the average lifetime of the $B^{+}$and $B^{0}$ mesons [4].

In order to compute the background contribution in bins of reconstructed $E_{\gamma}^{*}$, the theoretical spectrum needs to be transformed. This is done in two stages. First, it is convoluted with the ACCMM Fermi motion model to simulate the effect of the $b$-quark confinement in the $B$ meson [82. This is found to have little effect on the shape of the spectrum within a wide range of input parameters to the ACCMM model. Then 


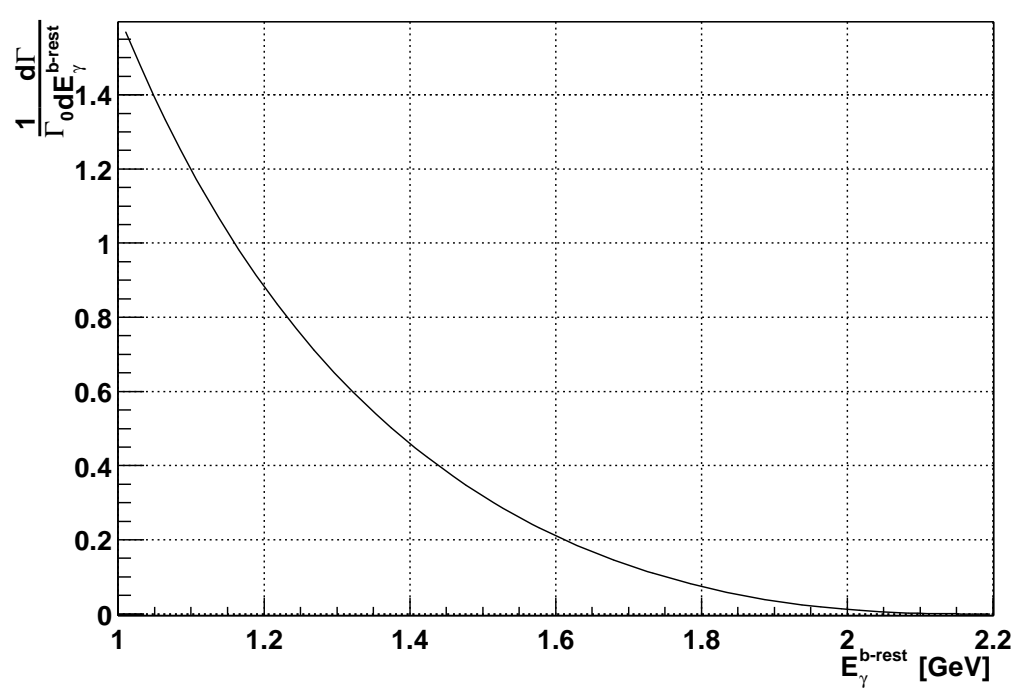

Figure 4.10: The $E_{\gamma}^{b-\text { rest }}$ spectrum for light-quark FSR, normalized to $\Gamma_{0}=1.17 \times 10^{-16} \mathrm{GeV}$.

the effects of the Lorentz boost from the $B$-meson to the $\Upsilon(4 S)$-meson rest frame and of the calorimeter resolution need to be computed. A combined parameterization of these, averaged over all values of $E_{\gamma}^{*}$, is taken from the signal simulation and convoluted with the spectrum. Integration over $100 \mathrm{MeV}$ intervals yields the branching fractions listed in Table 4.19,

These branching fractions are not themselves sufficient to compute the contribution from FSR events to the $B \bar{B}$ background; that requires the efficiency of the selection criteria as well. Since it is not possible to determine the efficiency without a full simulation of the FSR process, an estimate is made based on the the efficiency numbers from signal MC samples (Table 3.9 ) and from $B \bar{B}$ electron background samples (Table 4.14), with the latter adjusted for detector acceptance $(78.1 \%$ as described in Section 3.10) and for semileptonic- $B$-decay corrections (108\%). The average of these two inputs is taken to be the efficiency, with half of the difference assigned as a conservative systematic error.

The number of expected FSR background events in each bin of $E_{\gamma}^{*}$ is given in Table 4.19. The uncertainties on the yields are calculated by assuming that the 


\begin{tabular}{|c|c|c|c|}
\hline$E_{\gamma}^{*}(\mathrm{GeV})$ & $\mathcal{B}\left(\times 10^{-4}\right)$ & Efficiency $(\%)$ & $N_{\mathrm{FSR}}$ \\
\hline $1.6-1.7$ & 0.0468 & $0.33 \pm 0.15$ & $2.7 \pm 1.5$ \\
$1.7-1.8$ & 0.0286 & $0.49 \pm 0.23$ & $2.5 \pm 1.4$ \\
$1.8-1.9$ & 0.0160 & $0.55 \pm 0.25$ & $1.6 \pm 0.8$ \\
$1.9-2.0$ & 0.0079 & $0.64 \pm 0.23$ & $0.9 \pm 0.4$ \\
$2.0-2.1$ & 0.0032 & $0.85 \pm 0.32$ & $0.5 \pm 0.2$ \\
$2.1-2.2$ & 0.0010 & $0.83 \pm 0.40$ & $0.1 \pm 0.1$ \\
\hline
\end{tabular}

Table 4.19: The branching fraction, the selection efficiency and the expected number of background events from light-quark FSR, $N_{\mathrm{FSR}}$, in $100 \mathrm{MeV}$ bins of $E_{\gamma}^{*}$. The uncertainties are mainly systematic, as discussed in the text.

efficiency errors and a 30\% theoretical uncertainty on the spectrum calculation [83] are Gaussian errors.

\subsection{Overall Corrections to $B \bar{B}$ Simulation}

Table 4.20 gives the fractions of the various background components in the Monte Carlo simulation before any corrections are applied. The scaling of each component of the simulation as a result of the corrections described throughout this chapter are tabulated as a function of $E_{\gamma}^{*}$ in Table 4.22 Also included in this table are two additional systematic corrections, $\alpha_{\text {veto }}$ and $\alpha_{\text {semi }}$, which are corrections to the efficiency of the selection criteria on $B \bar{B}$ background events. They are applied to all the components as described in Sections 6.1.2 and 6.3.2.

Uncertainties on the scaling factors in Table 4.22 are systematic, reflecting the underlying uncertainties on the various corrections. The correlation matrix for these uncertainties is given in Table 4.23. The significant correlations among nearby bins are mainly because of the $\pi^{0}, \eta$ corrections, and to a lesser extent $\omega$, and $\eta^{\prime}$ corrections, being applied with respect to parent energies; each interval of parent energy contributes to several $E_{\gamma}^{*}$ bins. The antinucleon, $e^{ \pm}$and veto corrections are made as a function of the energy of the photon candidate.

The unsimulated FSR from light quarks described in Section 4.6 is treated as a special case: the expected number of FSR candidates is applied as an absolute 


\begin{tabular}{|c|c|c|c|c|c|c|c|}
\hline $\begin{array}{c}E_{\gamma}^{*} \\
(\mathrm{GeV})\end{array}$ & $\begin{array}{c}f_{\pi^{0}} \\
(\%)\end{array}$ & $\begin{array}{c}f_{\eta} \\
(\%)\end{array}$ & $\begin{array}{c}f_{\omega} \\
(\%)\end{array}$ & $\begin{array}{c}f_{\eta^{\prime}} \\
(\%)\end{array}$ & $\begin{array}{c}f_{n / p} \\
(\%)\end{array}$ & $\begin{array}{c}f_{e^{ \pm}} \\
(\%)\end{array}$ & $\begin{array}{c}f_{\text {other }} \\
(\%)\end{array}$ \\
\hline $1.6-1.7$ & 62.9 & 18.0 & 5.1 & 1.1 & 2.0 & 6.6 & 4.0 \\
$1.7-1.8$ & 63.7 & 18.0 & 4.2 & 1.0 & 1.8 & 7.8 & 3.0 \\
$1.8-1.9$ & 68.2 & 16.3 & 3.5 & 1.3 & 1.1 & 7.2 & 2.0 \\
$1.9-2.0$ & 67.1 & 18.0 & 2.3 & 0.8 & 2.6 & 6.9 & 2.0 \\
$2.0-2.1$ & 71.8 & 15.7 & 3.3 & 1.6 & 1.8 & 4.4 & 1.0 \\
$2.1-2.2$ & 70.0 & 20.1 & 2.9 & 0.5 & 1.6 & 1.8 & 2.0 \\
$2.2-2.3$ & 71.6 & 18.6 & 1.9 & 0.5 & 4.2 & 0.9 & 2.0 \\
$2.3-2.4$ & 58.2 & 23.9 & 3.8 & 1.6 & 8.2 & 2.2 & 2.0 \\
$2.4-2.5$ & 45.5 & 30.4 & 3.8 & 3.8 & 15.3 & 0.0 & 1.0 \\
$2.5-2.6$ & 56.8 & 17.7 & 0.0 & 3.9 & 19.7 & 0.0 & 1.0 \\
$2.6-2.7$ & 41.0 & 17.5 & 0.0 & 5.9 & 35.5 & 0.0 & 0.0 \\
$2.7-2.8$ & 14.1 & 0.0 & 0.0 & 0.0 & 71.5 & 0.0 & 14.0 \\
$2.8-2.9$ & 11.2 & 0.0 & 0.0 & 0.0 & 66.7 & 0.0 & 22.0 \\
$2.9-3.0$ & 0.0 & 0.0 & 0.0 & 0.0 & 100.0 & 0.0 & 0.0 \\
\hline
\end{tabular}

Table 4.20: The fractions $(f)$ of each component in the $B \bar{B}$ Monte Carlo simulation, after all selection criteria have been applied. 
additive correction. This correction leads to a change in the $B \bar{B}$ background of less than $1 \%$ for all $E_{\gamma}^{*}$.

The total number of expected $B \bar{B}$ background events, after all the corrections have been applied is given in Table 4.21. The values are listed for three alternative energy ranges that are considered for the extraction of the $B \rightarrow X_{s} \gamma$ signal yields (Section 3.4).

\begin{tabular}{|c|c|}
\hline$E_{\gamma}^{*}(\mathrm{GeV})$ & Total $B \bar{B}$ background \\
\hline $1.9-2.7$ & $1053.0 \pm 62.3$ \\
$2.0-2.7$ & $675.3 \pm 53.3$ \\
$2.1-2.7$ & $383.4 \pm 44.8$ \\
$2.2-2.7$ & $200.8 \pm 40.0$ \\
\hline
\end{tabular}

Table 4.21: The total $B \bar{B}$ background and its uncertainty in the four alternative ranges of photon energy. The uncertainty accounts for the correlations amongst the bins in Table 4.23,

Finally, the remaining sources of the $B \bar{B}$ backgrounds that have not been treated so far deserve a few words. The FSR photons from semileptonic $B$-meson decays, listed as photons with $B$ parentage in Table 4.1, are generated in the simulation by the PHOTOS package, as mentioned earlier. PHOTOS estimates are based on leadinglogarithmic $\mathcal{O}(\alpha)$ corrections. Following earlier studies in the literature, a $20 \%$ uncertainty can be assigned on these estimates [84]. Moreover, an independent uncertainty of $\sim 5 \%$ should be considered to account for having not applied the semileptonic$B$-decay corrections on these decays. However, even a combined uncertainty of $25 \%$ is too small to have any significant effects - the uncertainties from already-studied sources of background are much larger.

For the computation of the additional FSR background from light quarks in Section 4.6. $b \rightarrow u \bar{u} d+\gamma$ process has not been taken into consideration. As can be expected, the contribution from this process is much smaller than the $b \rightarrow c \bar{u} d+\gamma$. It becomes comparable only for $E_{\gamma}^{b-\text { rest }} \gtrsim 2 \mathrm{GeV}$ [80]. The contribution to the signal region is estimated to be less than a single event, which is neglected.

Since the background from $J / \psi$ decays is quite small, even if the simulated yields 
were wrong by a factor of two, the effect in the signal region would be minimal. Although a not-yet-explained excess in data has been observed at low $J / \psi$ momenta [85], the inclusive $J / \psi$ production is satisfactory in the simulation. Studies of the $J / \psi$ mass spectrum measured in $\mu^{+} \mu^{-}(\gamma)$ final state show that FSR generated by PHOTOS in $\ell^{+} \ell^{-}$final states is reasonable [86]. Finally, the decay rates for many of the lowmultiplicity radiative $J / \psi$ decays in the $B \bar{B}$ simulation are directly taken from earlier measurements [4].

The merged $\pi^{0}$ component of the background is insignificant. Due to the fine granularity of the calorimeter the photons from $\pi^{0}$ mesons in $B$-meson decays are mostly well separated. In the cases when the two photons merge into one shower, the criteria on the shower profile are very effective in indentifying them as such. The $\pi^{0}$ merging probability has been studied in $\tau^{ \pm}$decays and has been found to be about $16 \%$ higher in data compared to the simulation [87]. Given the size of the contribution, no correction is needed. 


\begin{tabular}{|c|c|c|c|c|c|c||c|c||c|}
\hline $\begin{array}{l}E_{\gamma}^{*} \\
(\mathrm{GeV})\end{array}$ & $\alpha_{\pi^{0}}$ & $\alpha_{\eta}$ & $\alpha_{\omega}$ & $\alpha_{\eta^{\prime}}$ & $\alpha_{\bar{n} / \bar{p}}$ & $\alpha_{e^{ \pm}}$ & $\alpha_{\text {veto }}$ & $\alpha_{\text {semi }}$ & $\alpha_{\text {all }}$ \\
\hline $1.6-1.7$ & $1.07 \pm 0.02$ & $0.77 \pm 0.06$ & $0.83 \pm 0.15$ & $0.92 \pm 0.29$ & $0.13 \pm 0.09$ & $1.09 \pm 0.04$ & $1.03 \pm 0.02$ & $1.08 \pm 0.01$ & $1.094 \pm 0.031$ \\
\hline $1.7-1.8$ & $1.08 \pm 0.02$ & $0.73 \pm 0.06$ & $0.83 \pm 0.15$ & $0.81 \pm 0.25$ & $0.10 \pm 0.07$ & $1.12 \pm 0.06$ & $1.03 \pm 0.02$ & $1.09 \pm 0.01$ & $1.111 \pm 0.032$ \\
\hline $1.8-1.9$ & $1.09 \pm 0.03$ & $0.68 \pm 0.07$ & $0.83 \pm 0.15$ & $0.79 \pm 0.25$ & $0.11 \pm 0.08$ & $1.15 \pm 0.08$ & $1.03 \pm 0.02$ & $1.09 \pm 0.01$ & $1.123 \pm 0.035$ \\
\hline $1.9-2.0$ & $1.08 \pm 0.03$ & $0.69 \pm 0.08$ & $0.83 \pm 0.15$ & $0.91 \pm 0.29$ & $0.06 \pm 0.04$ & $1.23 \pm 0.12$ & $1.03 \pm 0.02$ & $1.11 \pm 0.01$ & $1.124 \pm 0.037$ \\
\hline $2.0-2.1$ & $1.06 \pm 0.04$ & $0.78 \pm 0.09$ & $0.83 \pm 0.15$ & $1.64 \pm 0.54$ & $0.06 \pm 0.04$ & $1.31 \pm 0.15$ & $1.03 \pm 0.02$ & $1.07 \pm 0.01$ & $1.111 \pm 0.045$ \\
\hline $2.1-2.2$ & $1.05 \pm 0.05$ & $0.88 \pm 0.14$ & $0.83 \pm 0.15$ & $1.77 \pm 0.58$ & $0.05 \pm 0.03$ & $1.39 \pm 0.20$ & $1.03 \pm 0.02$ & $1.07 \pm 0.01$ & $1.104 \pm 0.051$ \\
\hline $2.2-2.3$ & $0.96 \pm 0.10$ & $0.92 \pm 0.16$ & $0.83 \pm 0.15$ & $1.77 \pm 0.58$ & $0.03 \pm 0.02$ & $1.39 \pm 0.20$ & $1.03 \pm 0.02$ & $1.11 \pm 0.01$ & $1.055 \pm 0.089$ \\
\hline $2.3-2.4$ & $0.73 \pm 0.27$ & $0.93 \pm 0.16$ & $0.83 \pm 0.15$ & $1.77 \pm 0.58$ & $0.04 \pm 0.03$ & $1.13 \pm 0.07$ & $1.01 \pm 0.01$ & $1.09 \pm 0.01$ & $0.833 \pm 0.174$ \\
\hline $2.4-2.5$ & $0.42 \pm 0.53$ & $0.93 \pm 0.16$ & $0.83 \pm 0.15$ & $1.77 \pm 0.58$ & $0.03 \pm 0.02$ & - & $1.01 \pm 0.01$ & $1.04 \pm 0.01$ & $0.623 \pm 0.257$ \\
\hline $2.5-2.6$ & $0.37 \pm 0.57$ & $0.93 \pm 0.16$ & - & $1.77 \pm 0.58$ & $0.04 \pm 0.03$ & - & $1.01 \pm 0.01$ & $1.08 \pm 0.01$ & $0.520 \pm 0.356$ \\
\hline $2.6-2.7$ & $0.44 \pm 0.51$ & $0.93 \pm 0.16$ & - & $1.77 \pm 0.58$ & $0.02 \pm 0.02$ & - & $1.01 \pm 0.01$ & $1.01 \pm 0.01$ & $0.469 \pm 0.217$ \\
\hline $2.7-2.8$ & $0.34 \pm 0.59$ & - & - & - & $0.02 \pm 0.01$ & - & $1.01 \pm 0.01$ & $1.13 \pm 0.03$ & $0.235 \pm 0.096$ \\
\hline $2.8-2.9$ & $0.96 \pm 0.06$ & - & - & - & $0.03 \pm 0.02$ & - & $1.01 \pm 0.01$ & $1.09 \pm 0.03$ & $0.383 \pm 0.022$ \\
\hline $2.9-3.0$ & - & - & - & - & $0.01 \pm 0.01$ & - & $1.01 \pm 0.01$ & $0.99 \pm 0.03$ & $0.007 \pm 0.005$ \\
\hline
\end{tabular}

Table 4.22: The weights $(\alpha)$ applied to each component of the $B \bar{B}$ MC simulation. The combined weight for all corrections is given in the last column, $\alpha_{\text {all }}$. The correction for the light-quark final state radiation is made independently as an absolute additive correction. 


\begin{tabular}{|c|c|c|c|c|c|c|c|c|c|c|c|c|c|}
\hline$E_{\gamma}^{*}(\mathrm{GeV})$ & 1.6 & 1.7 & 1.8 & 1.9 & 2.0 & 2.1 & 2.2 & 2.3 & 2.4 & 2.5 & 2.6 & 2.7 & 2.8 \\
\hline 1.6 & 1.000 & 0.463 & 0.384 & 0.328 & 0.281 & 0.220 & 0.145 & 0.091 & 0.088 & 0.057 & 0.065 & 0.040 & 0.020 \\
\hline 1.7 & & 1.000 & 0.439 & 0.376 & 0.303 & 0.242 & 0.161 & 0.091 & 0.081 & 0.050 & 0.056 & 0.033 & 0.018 \\
\hline 1.8 & & & 1.000 & 0.425 & 0.336 & 0.249 & 0.152 & 0.091 & 0.087 & 0.059 & 0.064 & 0.038 & 0.015 \\
\hline 1.9 & & & & 1.000 & 0.415 & 0.326 & 0.215 & 0.157 & 0.145 & 0.107 & 0.103 & 0.060 & 0.013 \\
\hline 2.0 & & & & & 1.000 & 0.398 & 0.299 & 0.258 & 0.252 & 0.202 & 0.206 & 0.116 & 0.012 \\
\hline 2.1 & & & & & & 1.000 & 0.384 & 0.235 & 0.191 & 0.118 & 0.139 & 0.060 & 0.009 \\
\hline 2.2 & & & & & & & 1.000 & 0.577 & 0.541 & 0.485 & 0.435 & 0.276 & 0.003 \\
\hline 2.3 & & & & & & & & 1.000 & 0.891 & 0.872 & 0.751 & 0.531 & 0.003 \\
\hline 2.4 & & & & & & & & & 1.000 & 0.892 & 0.752 & 0.569 & 0.030 \\
\hline 2.5 & & & & & & & & & & 1.000 & 0.740 & 0.605 & 0.004 \\
\hline 2.6 & & & & & & & & & & & 1.000 & 0.488 & 0.036 \\
\hline 2.7 & & & & & & & & & & & & 1.000 & 0.001 \\
\hline 2.8 & & & & & & & & & & & & & 1.000 \\
\hline
\end{tabular}

Table 4.23: The correlation matrix of the $B \bar{B}$ Monte Carlo sample between the bins of $E_{\gamma}^{*}$. The rows and columns are labeled by the value of $E_{\gamma}^{*}$ at the lower edge of the bin; all bin widths are $100 \mathrm{MeV}$. 


\section{Chapter 5}

\section{Photon Spectrum}

The $B \rightarrow X_{s} \gamma$ signal is extracted by subtracting the continuum and $B \bar{B}$ backgrounds from the on-resonance data. In this chapter, the steps followed in the subtraction are described, in the chronological order that they have been taken: scaling of the photon energy in the off-resonance data to account for the lower beam energies, subtraction in the control regions of the photon spectrum to validate the procedure, and unblinding of the signal, i.e. the extraction of the signal yields in the selected signal energy range.

\subsection{Scaling of Photon $E_{\gamma}^{*}$ in Off-resonance Data}

Since the off-resonance data is recorded about $40 \mathrm{MeV}$ below the mass of the $\Upsilon(4 S)$ resonance, the energies of the particles in continuum events will also be slightly lower in the off-resonance compared to the on-resonance data. To reduce the impact of this, particle distributions are assumed to be approximately the same in fractional momentum and per-event $E_{\gamma}^{*}$ values in the off-resonance data are scaled by a factor of $1058 / 1054$, i.e. the average ratio of the total c.m.-frame energies, whereever the off-resonance data is used to predict the on-resonance yields [67]. No $1 / s$ scaling is needed for the small difference in the cross sections, since this factor is absorbed into the luminosity measurement described in section 2.7.

All Monte Carlo samples (including continuum simulation) are generated at the $\Upsilon(4 S)$ resonance so no scaling is necessary for them. 


\subsection{Data in Control Regions}

An essential feature of this analysis, as mentioned various times in the earlier chapters, is its blind technique. Signal selection criteria are defined based on simulation and the simulation is corrected using independent control samples. Even the size of the systematic errors, which will be discussed in the following chapter, are initially estimated without any use of the signal region of the data.

In accordance with this blind philosophy, the subtraction of the backgrounds is initially performed in the control regions below and above the signal energy range. Table 5.1 lists the yields from on-resonance data with the total expected background subtracted.

\begin{tabular}{|c|r|c|c||c||c|}
\hline$E_{\gamma}^{*}(\mathrm{GeV})$ & \multicolumn{1}{|c|}{ Continuum } & $B \bar{B}$ & Data & Yield & MC Signal \\
\hline \hline $1.6-1.7$ & $76.5 \pm 25.5$ & $618.5 \pm 24.7$ & 738 & $42.3 \pm 37.3$ & $2.3 \pm 0.7$ \\
$1.7-1.8$ & $85.0 \pm 26.9$ & $550.6 \pm 22.5$ & 670 & $34.1 \pm 37.3$ & $3.5 \pm 0.9$ \\
$1.8-1.9$ & $85.0 \pm 26.9$ & $496.7 \pm 21.8$ & 629 & $46.9 \pm 36.8$ & $8.6 \pm 1.4$ \\
\hline $1.9-2.9$ & \multicolumn{5}{|c|}{ Signal region } \\
\hline $2.9-3.0$ & $42.5 \pm 19.0$ & $0.0 \pm 0.0$ & 75 & $32.5 \pm 20.9$ & $0.0 \pm 0.0$ \\
$3.0-3.1$ & $85.0 \pm 26.9$ & $0.5 \pm 0.4$ & 79 & $-6.5 \pm 28.3$ & $0.0 \pm 0.0$ \\
$3.1-3.2$ & $51.0 \pm 20.8$ & $0.5 \pm 0.5$ & 89 & $37.5 \pm 22.8$ & $0.0 \pm 0.0$ \\
$3.2-3.3$ & $93.5 \pm 28.2$ & $0.3 \pm 0.3$ & 76 & $-17.8 \pm 29.5$ & $0.0 \pm 0.0$ \\
$3.3-3.4$ & $119.0 \pm 31.8$ & $1.0 \pm 0.7$ & 71 & $-49.0 \pm 32.9$ & $0.0 \pm 0.0$ \\
\hline
\end{tabular}

Table 5.1: The background estimation compared to the on-resonance data in the control regions. The background is the sum of the continuum contribution estimated from off-resonance data and $B \bar{B}$ contribution from simulation with all corrections (Table 4.22) applied. The yield from the subtraction of the total background from the on-resonance data, and the expected number of signal events from the default signal model are given. Continuum and expected signal uncertainties are statistical, while $B \bar{B}$ errors also include the systematic uncertainties of the correction factors. The uncertainties in the yields are statistical only; the systematic uncertainties are equal to the $B \bar{B}$ uncertainties.

The high $E_{\gamma}^{*}$ region, $2.0<E_{\gamma}^{*}<3.4 \mathrm{GeV}$, provides a check of the continuum subtraction, since the $B \bar{B}$ background in this region is completely negligible. The total yield after background subtraction is $-3.3 \pm 60.9$ events, consistent with zero. 
However the accuracy of the test is limited by the size of the sample: with all the selection criteria applied, there are only 390 events on-resonance data events in this region. To improve the statistics, various selection criteria are switched on and off and the test is repeated. In each case the subtracted yield is consistent with zero. For example, if the tagging criteria are waived, the yield after the subtraction is $(-0.3 \pm 0.6) \%$ of the number of on-resonance data events.

The comparison in the $B \bar{B}$ background control region, $1.6<E_{\gamma}^{*}<1.9 \mathrm{GeV}$, is more complicated due to the correlations between the predicted $B \bar{B}$ background events across the bins. Moreover, a small signal contribution is expected in this region: even the default signal model, which is known to underestimate the total branching fraction, predicts $17 \pm 2$ events. Ignoring this contribution, the yield after subtraction is $125 \pm 64$ (stat) \pm 54 (syst) events. The significance of this deviation from zero is 1.5 standard deviations. If the tagging criteria are waived to improve the statistics of the sample, this value goes down to 1.1 standard deviations.

\subsection{Signal Yields}

After the results of the tests in both control regions are found to be satisfactory, the signal region of the data is ready to be unblinded. However, there is one last decision to be made, the selection of the signal energy range, which was tentatively set to $2.0<E_{\gamma}^{*}<2.7 \mathrm{GeV}$ as a reasonable starting point (Section 3.4).

For this selection, estimates are made of the expected uncertainty on the branching fraction measurement, which take into account all the systematic uncertainties (these will be discussed in Chapter [6] using the unblinded data, but all of the mentioned effects have first been evaluated with Monte Carlo studies) and the uncertainties associated with the dependence on the signal model used (Chapter 7).

As can be expected, these estimates are highly dependent on the assumed value of the total branching fraction. For instance, while the predictions for the total uncertainty in the $2.0<E_{\gamma}^{*}<2.7 \mathrm{GeV}$ range from the default (KN480) signal model, which assumes $\mathcal{B}\left(B \rightarrow X_{s} \gamma\right)=2.6 \times 10^{-4}$, have been a total uncertainty of about $16 \%$, the KN465 model prediction, which assumes $\mathcal{B}\left(B \rightarrow X_{s} \gamma\right)=4.0 \times 10^{-4}$, is 
approximately $14 \%$.

To resolve this issue, a partial unblinding technique is used. First the higher part of the spectrum is unblinded, $E_{\gamma}^{*}>2.1 \mathrm{GeV}$. Using the observed number of events in this higher end of the spectrum, the assumed branching fraction for the KN465 model is found to be more realistic. With this finding, the energy range range is selected to be $1.9<E_{\gamma}^{*}<2.7 \mathrm{GeV}$. After this selection the lower part of the spectrum is also unblinded. The signal yields are tabulated in Table 5.2 and the whole spectrum is shown in Figure 5.1 .

\begin{tabular}{|c|c|c|c||c|}
\hline$E_{\gamma}^{*}(\mathrm{GeV})$ & Continuum & $B \bar{B}$ & Data & Yield \\
\hline \hline $1.9-2.0$ & $85.0 \pm 26.9$ & $377.9 \pm 18.6$ & 513 & $50.1 \pm 35.1$ \\
$2.0-2.1$ & $34.0 \pm 17.0$ & $292.1 \pm 16.8$ & 423 & $96.9 \pm 26.7$ \\
$2.1-2.2$ & $51.0 \pm 20.8$ & $182.8 \pm 12.7$ & 371 & $137.2 \pm 28.4$ \\
$2.2-2.3$ & $68.0 \pm 24.0$ & $98.2 \pm 10.8$ & 301 & $134.8 \pm 29.6$ \\
$2.3-2.4$ & $85.0 \pm 26.9$ & $66.4 \pm 14.9$ & 341 & $189.6 \pm 32.6$ \\
$2.4-2.5$ & $68.0 \pm 24.0$ & $21.3 \pm 9.3$ & 288 & $198.7 \pm 29.4$ \\
$2.5-2.6$ & $76.5 \pm 25.5$ & $11.5 \pm 8.1$ & 235 & $147.0 \pm 29.8$ \\
$2.6-2.7$ & $59.5 \pm 22.5$ & $3.4 \pm 2.0$ & 151 & $88.1 \pm 25.6$ \\
$2.7-2.8$ & $85.0 \pm 26.9$ & $1.4 \pm 0.9$ & 86 & $-0.4 \pm 28.4$ \\
$2.8-2.9$ & $42.5 \pm 19.0$ & $1.5 \pm 0.8$ & 67 & $23.0 \pm 20.7$ \\
\hline
\end{tabular}

Table 5.2: The unblinded data, the estimated background, and the extracted signal yield. The continuum contribution to the background is estimated from off-resonance data; the $B \bar{B}$ contribution is from simulation with all corrections (Table 4.22) applied. Continuum uncertainties are statistical, while $B \bar{B}$ errors also include the systematic uncertainties of the correction factors. The uncertainties in the signal yields are statistical only; the systematic uncertainties are equal to the $B \bar{B}$ uncertainties.

The total signal yield in the selected energy range of $1.9<E_{\gamma}^{*}<2.7 \mathrm{GeV}$ is $1042.8 \pm 84.3 \pm 62.3$ events (Table 5.3) , where the first uncertainty is statistical and the second one is from the uncertainty on the subtracted $B \bar{B}$ contribution (Table 4.21). 


\begin{tabular}{|l|rllll|}
\hline$E_{\gamma}^{*}(\mathrm{GeV})$ & \multicolumn{5}{|c|}{ Signal Yield (events) } \\
\hline $1.9-2.7$ & 1042.9 & \pm & 84.3 & \pm & 62.3 \\
$2.0-2.7$ & 992.6 & \pm & 76.6 & \pm & 53.3 \\
$2.1-2.7$ & 895.5 & \pm & 71.8 & \pm & 44.8 \\
$2.2-2.7$ & 758.2 & \pm & 65.9 & \pm & 40.0 \\
\hline
\end{tabular}

Table 5.3: Extracted total signal yields in four ranges of $E_{\gamma}^{*}$. The first error is statistical, the second is from $B \bar{B}$ systematics.

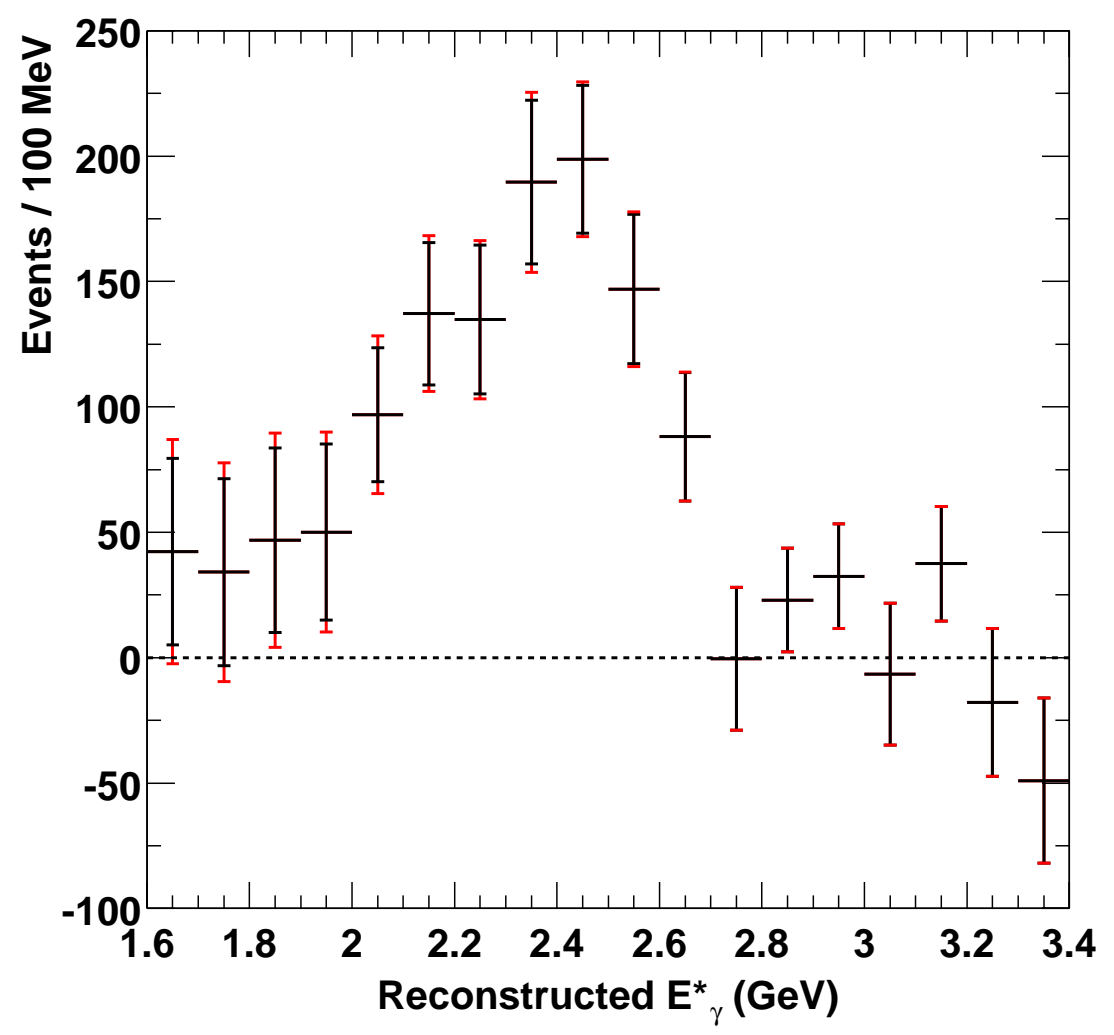

Figure 5.1: Photon energy spectrum after background subtraction, uncorrected for efficiency. Error bars include statistical (dominant) and $B \bar{B}$ systematic uncertainties, added in quadrature. 


\section{Chapter 6}

\section{Systematic Corrections and Uncertainties}

For the inclusive analysis of a rare signal that is heavily overwhelmed by backgrounds, a multitude of different sources of possible systematic biases and uncertainties are unavoidable. From the calibration of the detector to the tuning of the Monte Carlo simulation, many effects have to be studied carefully and quantified. An essential difficulty in this assessment is the blind nature of the analysis. As mentioned in Sections 3.4 and 5.3, all the systematic uncertainties need to be estimated initially without using the signal region of the data, so that the optimized energy range could be selected for the final energy range selection.

After the data in unblinded, the systematic uncertainties are recomputed. The estimates have been found to be quite accurate, therefore in this chapter, only the finalized figures, as summarized in Table 6.1, are given. The photon energy range of $2.0<E_{\gamma}^{*}<2.7 \mathrm{GeV}$ is chosen as a representative energy range to give an appreciation of the magnitudes of various effects considered. On the other hand, it should be emphasized that the computations are done separately for each signal range (i.e. also for $1.9-2.7 \mathrm{GeV}, 2.1-2.7 \mathrm{GeV}$ and $2.2-2.7 \mathrm{GeV}$ ) and used in extracting the results as a function of minimum energy (Chapter [7). 


\begin{tabular}{|l|l|c|}
\hline Section & \multicolumn{1}{|c|}{ Systematic } & Uncertainty \\
\hline \hline 6.1 .1 & Shower detection and selection & $3.4 \%$ \\
6.1 .2 & $\pi^{0}(\eta)$ veto & $0.5 \%$ \\
6.2 & Event topology criteria & $3.3 \%$ \\
6.3 .1 & Lepton identification & $2.2 \%$ \\
6.3 .2 & Semileptonic- $B$-decay correction & $3.0 \%$ \\
6.4 & Normalization & $1.1 \%$ \\
6.5 & $B \bar{B}$ background estimation & $5.7 \%$ \\
6.6 & Signal MC precision & $1.1 \%$ \\
\hline \hline Total & & $8.5 \%$ \\
\hline
\end{tabular}

Table 6.1: Systematic uncertainties, expressed relative to $\mathcal{B}\left(B \rightarrow X_{s} \gamma\right)$ for $2.0<E_{\gamma}^{*}<2.7 \mathrm{GeV}$. The left column gives the subsection in which the estimation of a contribution is discussed in detail. The total is the quadratic sum. Note that the $B \bar{B}$ uncertainty includes an extra component to take into account the correlated signal-background uncertainties from the semileptonic correction.

\subsection{Photon Selection}

\subsubsection{Shower Identification and Selection}

The photon selection and the measurement of its energy are the main components of the inclusive analysis. As such many systematic effects have been carefully reviewed. A summary of the uncertainties are given in Table 6.2.

The photon detection and reconstruction efficiency is measured with $\tau^{+} \tau^{-}$events from a $55 \mathrm{fb}^{-1}$ subsample of the data 88 . The ratio of decays with one and two $\pi^{0}$ mesons in data is compared to the simulation (Equation 6.1 is computed as a function of the $\pi^{0}$ energy).

$$
R=\frac{N^{\text {data }}\left(\tau \rightarrow h^{ \pm} \pi^{0} \pi^{0}\right) / N^{\text {data }}\left(\tau \rightarrow h^{ \pm} \pi^{0}\right)}{N^{M C}\left(\tau \rightarrow h^{ \pm} \pi^{0} \pi^{0}\right) / N^{M C}\left(\tau \rightarrow h^{ \pm} \pi^{0}\right)}
$$

The photon efficiency is then adjusted to obtain $R=1.0$. It is found that for isolated high energy photons no correction is required [89]. The statistical precision of this 


\begin{tabular}{|l|c|}
\hline Source & Uncertainty \\
\hline Shower reconstruction efficiency & $\pm 2.5 \%$ \\
Second moment requirement & $\pm 0.7 \%$ \\
Shower isolation requirement & $\pm 2.0 \%$ \\
Energy scale & $\pm 0.5 \%$ \\
Energy resolution & $\pm 0.5 \%$ \\
\hline Total & $\pm 3.4 \%$ \\
\hline
\end{tabular}

Table 6.2: Systematic effects associated with detection and measurement of the high-energy photon candidates and the corresponding uncertainties in $\mathcal{B}\left(B \rightarrow X_{s} \gamma\right)$.

procedure gives a $2.5 \%$ systematic uncertainty.

A small sample of virtual Compton scattering (VCS) photons (Appendix B) is used to check the shower shape criteria. The efficiencies for the second moment requirement are $98.8 \%$ (with negligible error) and (99.5 \pm 0.02$) \%$ in data and in simulation respectively. No corrections to the signal efficiency are applied for this difference: the effect is largely offset by the correction on the $\pi^{0} / \eta$ vetoes (Section 6.1.2). As for the other shower shape criterion, the lateral moment, although significant discrepancies have been identified in other BaBar analyses [90], these become much less profound after the second moment requirement and mostly integrate out. A total systematic uncertainty of $0.7 \%$ is assigned for these effects.

The possible systematic errors associated with the shower isolation requirement has been assessed in the earlier BABAR analysis of the exclusive $B \rightarrow K^{*} \gamma$ decay [91]. Photons from radiative Bhabha events are embedded into samples of $B \bar{B}$ Monte Carlo and $B \rightarrow D \pi$ data events with a technique very similar to the one used for the determination of the electron efficiencies (Section 4.4.2): the digitized EMC signals for the photon showers are mixed with the signals of the $B \bar{B}$ events, the reconstruction algorithms are rerun and the distance from the embedded photon to the nearest other shower is computed. The resulting distibutions of isolation distance in data and simulation show remarkable agreement: both peak at around $50 \mathrm{~cm}$ and extend up to $200 \mathrm{~cm}$ (very similar to the signal distribution of this quantity as shown in 
Figure 3.2). The efficiency of requiring the isolation to be at least $25 \mathrm{~cm}$ is found to be slightly higher in the simulation and the small difference of $2 \%$ is assigned as the systematic error.

Since the extraction of the photon energy spectrum is one of the primary goals of this analysis, the correct determination of the energy scale is exteremely important. For this reason, multiple methods have been used to independently measure this quantity. These include the measurement of the $\eta$-meson mass in symmetric $\eta \rightarrow \gamma \gamma$ decays, the comparison of the beam energy with the energy of the reconstructed $B$ mesons in exclusive $B \rightarrow K^{*} \gamma$ events, and studies using the VCS samples. Taking into account various small discrepancies observed in these studies, a conservative uncertainty of $\pm 0.5 \%$ is assigned on the photon energy scale (for data relative to simulation). In order to determine the effect of this uncertainty on the branching fraction measurement, the photon energies in the Monte Carlo signal samples are shifted by integer multiples of $\pm 0.5 \%$. The resulting change in the signal selection efficiency shows a rough linear dependence on the amount of shift. Following this linear relationship, a $\pm 0.5 \%$ uncertainty is assigned as the energy scale error. It should be noted that small shifts have also been observed in the fits to the $\pi^{0}$ and $\eta$ mass spectra (as discussed in Section 4.2), but the $\pi^{0}(\eta)$ correction factors fix this problem.

As discussed in Section 2.4, the energy resolution of the calorimeter is determined from many different processes. Further studies have been performed using the exclusive $K^{*} \gamma$ and the VCS samples. The simulation agrees well with the data for all of these studies. To account for the small differences that are observed in the VCS samples, a study similar to the one described in the previous paragraph is done, this time applying a $1 \%$ smearing to the energies of the photons in the signal MC samples. The resulting change in the efficiency is approximately $0.5 \%$ which is assigned as an additional systematic.

Finally the same VCS studies also provide information on the low-end tail of the calorimeter resolution function, which is fit to a Novosibirsk function. The parameter describing the tail of the Novosibirsk function is found to slightly different in VCS data and simulation, however the effects on the signal efficiency can be neglected. 
However, these differences are recorded and are later used in the computation of the corrections to the second moment of the photon spectrum (Section [7.3).

\subsection{2 $\quad \pi^{0}$ and $\eta$ Vetoes}

The veto described in Section 3.3 .3 forms the invariant mass of the high-energy signal photon with any other good photon above a low energy threshold and discards those combinations that fall within a window around the nominal $\pi^{0}$ or $\eta$ mass. In addition to discarding high-energy photon candidates which are truely from $\pi^{0}$ or $\eta$ mesons, this technique also discards a significant number of photon candidates with combinatorics that fall within the veto window.

There are two effects that result in a correction to the veto efficiency and an associated systematic uncertainty. First, the fitted lineshape for the $\pi^{0}$ mesons is found to be slightly narrower in the simulation than in data, causing the estimated number of vetoed events to be overestimated. Second, the spectrum of low-energy showers in the simulation is found to be slightly overestimated compared to data. This also leads to an overestimation of the number of vetoed events. The veto predominantly discards $B \bar{B}$ background; only the second effect is relevant for signal, where it has little impact on the efficiency.

The lineshape is fitted in the $\pi^{0}$ and $\eta$ control samples described in detail in Section 4.2 These fits are used to estimate the relative efficiency of the veto in data and simulation. It is observed that the relative efficiency correction is approximately independent of $E_{\gamma}^{*}$ over the range of interest and that the number of background $B \bar{B}$ events containing a real $\pi^{0}(\eta)$ expected to pass the veto is increased by a factor of $1.02 \pm 0.02(1.01 \pm 0.01)$.

\begin{tabular}{|cccc|c|}
\hline \multicolumn{3}{|c|}{$E_{\gamma}$ bin } & Data/MC \\
\hline 0.03 & to & 0.10 & $\mathrm{GeV}$ & $0.88 \pm 0.02$ \\
0.10 & to & 0.25 & $\mathrm{GeV}$ & $0.95 \pm 0.05$ \\
\hline
\end{tabular}

Table 6.3: The ratio of the number of low-energy photons per event between data and Monte Carlo simulation, vs. photon energy in the lab frame. The difference is negligible above the energies shown. 
The spectrum of low energy photons is measured in the data and the simulation before the veto is applied. Table 6.3 gives the ratio of the two spectra in bins of the photon energy. The low-energy photon spectrum is corrected by randomly discarding a fraction of the low-energy photons that form a combinatoric within the veto window, according to the measured ratio. This results in an increase in the number of $B \bar{B}$ events that pass the veto by a factor of $1.02 \pm 0.01$ for the region $E_{\gamma}^{*}<2.3 \mathrm{GeV}$. The effect in the region $E_{\gamma}^{*}>2.3 \mathrm{GeV}$ is negligible due to the higher low energy photon threshold of $150 \mathrm{MeV}$ used in the veto. The correction is found to be independent of $E_{\gamma}^{*}$ for the region below $2.3 \mathrm{GeV}$.

For the $B \bar{B}$ sample, $80 \%(45 \%)$ of the events rejected by the $\pi^{0}(\eta)$ veto are due to real $\pi^{0}(\eta)$ mesons, the remaining 20\%(55\%) vetoed due to combinatorics. The two corrections weighted for this composition result in a total veto correction of $\alpha_{\text {veto }}=$ $1.03 \pm 0.02$ for $E_{\gamma}^{*}$ below $2.3 \mathrm{GeV}$ and $\alpha_{\text {veto }}=1.01 \pm 0.01$ above $2.3 \mathrm{GeV}$ (Table 4.22).

For the signal, the combinatoric effect results in a correction of 1.015 below $2.3 \mathrm{GeV}$, and no correction above $2.3 \mathrm{GeV}$. This results in an average efficiency correction of $1.005 \pm 0.005$. This correction largely cancels the effect of the second-moment data-simulation discrepancy discussed in the previous subsection (Section 6.1.1). Therefore the signal yields need not be modified, but the uncertainty $(0.5 \%)$ is taken as a systematic error.

\subsection{Event Topology Selection}

The distributions for the event topology variables, particularly the output of the Fisher discriminant, rely on information from all good tracks and EMC showers. Therefore even small differences between data and simulation may cause significant differences in these distributions and a bias in the selection efficiencies. Specifically, the Fisher distribution can be sensitive to details of the $X_{s}$ fragmentation, or to the modeling of the decay of the other $B$ meson, which provides the tagging lepton.

These possibilities are investigated using two methods. In the first method (Section 6.2.1), the real and simulated distributions of the Fisher discriminant output are 
compared for an inclusive $\pi^{0}$ control sample and the impact of any observed differences on the signal efficiency is computed. The second method (Section 6.2.2) relies on measuring the dependence of the signal efficiency on the $X_{s}$ fragmentation model and estimating the size of any biases that it can lead to.

The two methods may in part be measuring the same systematic effect. Most conservatively, the uncertainties determined from both methods are added in quadrature. This results in an overall systematic uncertainty of $3.3 \%$.

\subsubsection{Fisher Discriminant in Data and Simulation}

Inclusive $\pi^{0}$ control samples are used to check the modeling of the Fisher discriminant output in the simulation against the data. This allows the computation of the systematic error before the unblinding of the $B \rightarrow X_{s} \gamma$ signal. The method relies on replacing the signal photon with a $\pi^{0}$-meson as the primary high-energy particle of interest, and defining all the event topology variables with respect to the momentum of the $\pi^{0}$-meson. The Fisher discriminant output, $F(\boldsymbol{x})$, is recomputed with such $\pi^{0}$-centered $R_{2}^{\prime} / R_{2}^{*}$ and energy cone variables as inputs, while the parameters of the function are kept at their original values. The distribution for this recomputed Fisher discriminant output can be compared between continuum-subtracted on-resonance data and $B \bar{B}$ simulation, in a similar way to that of the yield studies described in Section 4.2, and the conclusions applied to the photon analysis.

The selection criteria used in creating the $\pi^{0}$ sample strongly parallel the standard requirements of the photon analysis. After the QED continuum rejection criteria have been applied (Section 3.2), $\pi^{0}$ candidates are reconstructed by combining any highenergy photon $\left(E_{\gamma}^{*}>1.0 \mathrm{GeV}\right)$ with any other good photon in the event and keeping only those combinations that would fail the $\pi^{0}$ veto (the same energy and mass requirements as detailed in Section 3.3.3). When more than one combination exists for a given high-energy photon, the combination with the highest-energy secondary photon is selected. This happens for well under one-tenth of the $\pi^{0}$ candidates after all the following selection criteria have been applied: 
- All photon quality and shower shape and isolation criteria of the standard analysis (Sections 3.3.1 and 3.3.2) on the primary (higher $E^{*}$ ) photon. The primary photon must also survive the $\eta$ veto (Section 3.3.3).

- The same second moment and lateral moment criteria on the secondary photon.

- $2.0<E_{\pi^{0}}^{*}<2.7 \mathrm{GeV}$ and $R_{2}^{*}<0.55$.

- All lepton tag criteria as in Section 3.7. but with the requirement on the lepton direction defined with respect to the c.m.momentum of the reconstructed $\pi^{0}$ candidate.

- $N_{\text {mult }} \geq 4.5$ (Section 3.9) .

The resulting $\pi^{0}$ sample is very clean: in the Monte Carlo simulation $97.5 \%$ of the selected primary photons are from true $\pi^{0}$ mesons. Moreover, for $95 \%$ of the events with true $\pi^{0}$ mesons, the tag lepton originates from the other $B$ meson. Therefore, except for the differences in the energy spectrum $\left(E_{\pi^{0}}^{*}\right.$ distribution is different from the $E_{\gamma}^{*}$ distribution), this sample really mimics the $B \rightarrow X_{s} \gamma$ signal sample.

A comparison of the distributions for the recomputed Fisher discriminant output in the $\pi^{0}$ sample is shown in figure 6.1. The agreement in the general features is excellent between the data and the simulation. To quantify the small differences, the histograms are fit to Crystal Ball functions [68. The simulated values for the peak position (labeled "mean" in the figure) and the width of the Gaussian part are consistent with the data. In the data sample, the tail constitutes $2 \%$ more of the total distribution than in the simulation.

It is reasonable to assume that the small difference in the tail is an artifact of the off-resonance data subtraction. In contrast to the photon energy, which is scaled for off-resonance events (Section 5.1), the Fisher discriminant has no corrections that would account for the low beam energy of the off-resonance data. Hence, some small discrepancies in the subtraction are to be expected, especially considering the strong correlation between the Fisher discriminant and the photon energy.

This assumption about the origin of the slightly larger tail in data can be independently tested by a comparison of the regular Fisher discriminant for the continuum 

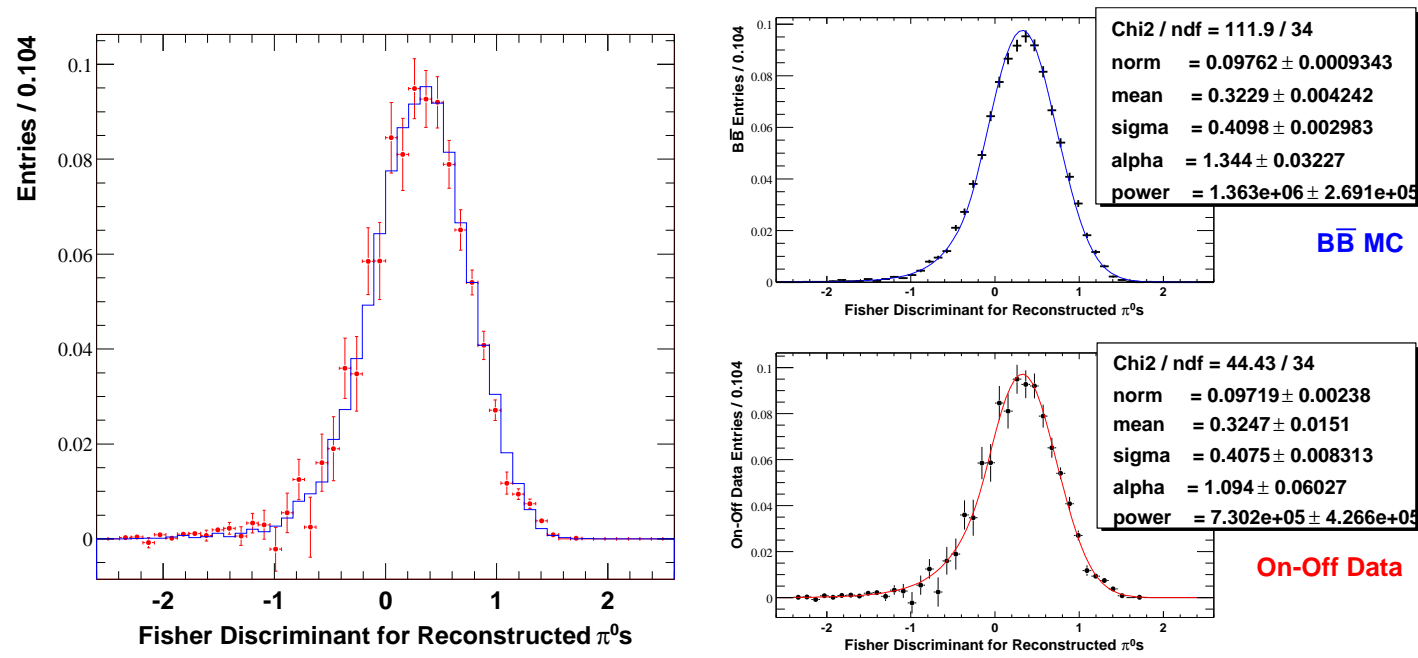

Figure 6.1: Distributions for the Fisher discriminant output recomputed in $\pi^{0}$ the control sample, normalized to unit area. The left plot compares the off-resonance subtracted on-resonance data (red points) with the $B \bar{B}$ simulation (blue line). The right plots show Crystal Ball functions 68 fitted to the Monte Carlo simulation (top) and data (bottom) distributions.

simulation and the off-resonance data. Figure 6.2 shows that the continuum MC distribution has a larger low-end tail compared to the data. Since the continuum MC sample is generated at the energy of the on-resonance data, this observation strongly supports the assumption. Moreover, the comparison suggests that the feature observed is perhaps due to a small systematic shift in the distribution, rather than just a discrepancy in the low-end tail.

In order to determine the size of the systematic uncertainty, a study is done of the dependence of the signal efficiency on a possible systematic shift in discriminant outputs between data and simulation in the $\pi^{0}$ control sample. Figure 6.3 shows how such a systematic shift would change the simulated efficiency of the Fisher discriminant criterion for the $B \rightarrow X_{s} \gamma$ signal. Since no statistically significant shift is observed in the control sample, the uncertainty on the difference between the peak positions of data and of Monte Carlo (0.0157) is taken as a conservative estimate of 


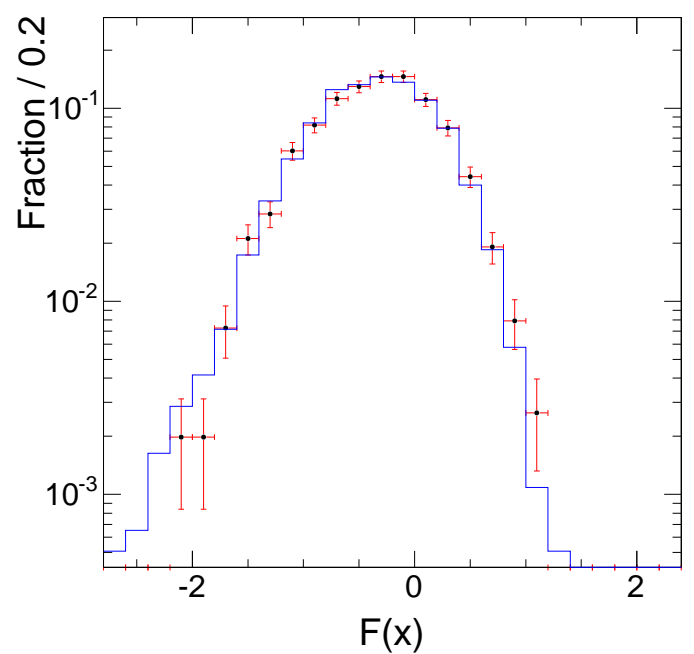

Figure 6.2: Distributions for the regular Fisher discriminant output for continuum simulation (blue histogram) and off-resonance data (red data points), normalized to unit area. The plot is made for a sample of events that satisfy the standard selection criteria of Chapter 3, with two exceptions that reduces the impact of the non-simulated QED component of the continuum backgrounds: no $\pi^{0} / \eta$ vetoes applied and $N_{m u l t} \geq 6.0$.

a possible shift. A shift of this size would result in a $3.0 \%$ systematic error.

It is important to emphasize that any differences in the Fisher discriminant shape would also affect the amount of $B \bar{B}$ background subtracted. However, the analysis is robust against such effects, since the correction factors for the $\pi^{0}$ and $\eta$ components of the $B \bar{B}$ background are derived after the selection criterion on the Fisher discriminant is applied. Thus a systematic problem in the modelling of the discriminant would be mostly absorbed into these correction factors.

As a final check, an estimate is made of the impact of taking the small difference in the low-end tail by itself and not as consequence of an overall shift. In the standard photon analysis, the efficiency of the $F(\boldsymbol{x})>0.575$ requirement on the signal sample is $(50.21 \pm 0.58) \%$, if all the other selection criteria are applied first. The $\pi^{0}$ control sample has a lower average Fisher discriminant output than the signal photon sample, so the same efficiency is obtained for a lower value of the minimum accepted disciminant output: $(50.21 \pm 0.34) \%$ of the total distribution is above 0.305 , as determined 


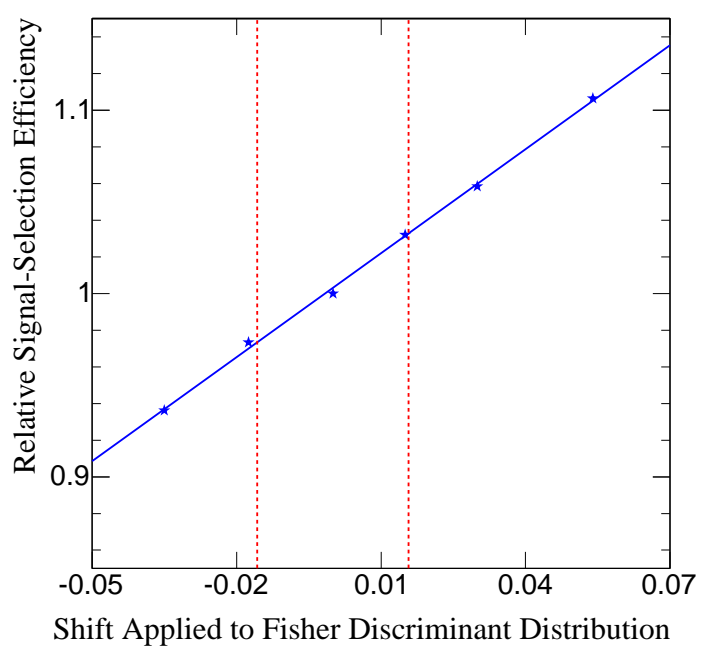

Figure 6.3: Relative change in the overall efficiency of the selection criteria on the $B \rightarrow X_{s} \gamma$ signal events as the Fisher discriminant distribution is systematically shifted. The fitted line, with a slope of 1.8906 , is obtained from linear regression $(\mathrm{R}=0.9987)$. A shift of 0.0157 would result in a $3.0 \%$ change in the signal efficiency.

by integrating the Crystal Ball function fitted to the Monte Carlo distribution. On the other hand the fraction of the integral above 0.305 is $(48.86 \pm 1.14) \%$ for the fitted function to $\pi^{0}$ data sample. If the tail of the photon data were to behave like the $\pi^{0}$ sample, this would correspond to a $(2.7 \pm 2.4) \%$ bias on the signal efficiency, covered by the $3.0 \%$ uncertainty already assigned.

\subsubsection{Altering the Fragmentation Model}

A complementary approach is to directly check how alterations in the $X_{s}$ fragmentation model change the efficiency of the selection criteria on the signal MC samples. Although this is a systematic check of all the selection criteria together, it is likely that any sensitivity to fragmentation is directly related to the event topology requirements. Hence, it is described in this subsection.

The signal MC simulation of the $X_{s}$ fragmentation is based on Jetset. Unfortunately the distributions of both the multiplicity and the ratio of the numbers of 
neutral to charged pions among the $X_{s}$ fragmentation products differ significantly for data and simulation [28]. As mentioned in Section [1.9, this is a significant source of systematic uncertainty for semi-inclusive analyses which rely on reconstructing the $X_{s}$ system. A key advantage of the fully inclusive measurement is that any sensitivity to the fragmentation model arises solely from the sensitivity of the selection efficiency on these quantities.

Most of the variables used in the event selection are unlikely to exhibit any dependence on the $X_{s}$ fragmentation, since they do not rely on any information from the $X_{s}$ system. Among all the selection variables, the only ones that contain some $X_{s}$ information are the event topology, the missing energy and the effective multiplicity. Since the dependence of the $N_{\text {mult }}$ requirement on $X_{s}$ multiplicity has already been studied in Section 3.9 this section focuses on the $R_{2}^{*}$, the Fisher discriminant and the missing energy variables.

\begin{tabular}{|r|r|c|c|c|c|c|}
\hline \multirow{4}{*}{ Mult } & \multicolumn{6}{|c|}{$E_{\gamma}^{*}$ Interval $(\mathrm{GeV})$} \\
\cline { 2 - 7 } & \multicolumn{2}{|c|}{$2.0-2.2$} & \multicolumn{2}{|c|}{$2.2-2.4$} & \multicolumn{2}{|c|}{$2.4-2.7$} \\
\cline { 2 - 7 } & Evts & Eff. (\%) & Evts & Eff. (\%) & Evts & Eff. (\%) \\
\hline 2 & 5621 & $1.81 \pm 0.18$ & 11743 & $2.55 \pm 0.15$ & 13959 & $3.18 \pm 0.15$ \\
3 & 16522 & $1.86 \pm 0.11$ & 30988 & $2.85 \pm 0.10$ & 28098 & $3.44 \pm 0.11$ \\
4 & 21589 & $1.91 \pm 0.09$ & 26001 & $2.74 \pm 0.10$ & 12483 & $3.20 \pm 0.16$ \\
5 & 20786 & $1.85 \pm 0.09$ & 16360 & $2.80 \pm 0.13$ & 4548 & $2.92 \pm 0.26$ \\
$\geq 6$ & 29924 & $1.64 \pm 0.07$ & 11957 & $2.38 \pm 0.14$ & 1612 & $2.30 \pm 0.38$ \\
\hline All & 94442 & $1.80 \pm 0.04$ & 97049 & $2.72 \pm 0.05$ & 60700 & $3.26 \pm 0.07$ \\
\hline
\end{tabular}

Table 6.4: Dependence of the combined efficiency of event topology and lepton tagging criteria on the $X_{s}$ final-state multiplicity, as measured in three intervals of $E_{\gamma}^{*}$ in the flat Monte Carlo signal samples. As described in Section 2.7, these samples are generated to have a flat $E_{\gamma}$ distribution (in the $B$-meson rest frame), but fragmented with the usual Jetset parameterizations for $X_{s}$. The efficiency values are given with respect to the events, whose number are listed in "Evts" columns, that have already satisfied the preselection and photon selection criteria.

Table 6.4 shows the combined efficiency of the event topology and the lepton tagging criteria, as a function of the $X_{s}$ multiplicity, which is defined as the total number of charged and neutral kaons and pions (excluding pions from $K_{S}^{0}$ decays) 


\begin{tabular}{|c|r|c|}
\hline$r=n_{\pi^{0}} / n_{\pi^{ \pm}}$ & Events & Efficiency $(\%)$ \\
\hline$r=0$ & 27711 & $3.23 \pm 0.11$ \\
$0.0<r \leq 0.5$ & 16931 & $2.72 \pm 0.13$ \\
$0.5<r \leq 1.0$ & 19667 & $2.97 \pm 0.13$ \\
$1.0<r<\infty$ & 5613 & $2.76 \pm 0.23$ \\
$n_{\pi^{ \pm}}=0$ & 11548 & $2.85 \pm 0.16$ \\
\hline Total & 81470 & $2.97 \pm 0.06$ \\
\hline
\end{tabular}

Table 6.5: The number of events and the combined efficiency of the event topology and lepton tagging criteria on neutral-to-charged-pion-multiplicity ratio for the default (KN480) Monte Carlo simulation. Pions from $K_{S}^{0}$ decay are not counted. The efficiencies apply to events that have already satisfied the preselection, photon selection and $2.0<E_{\gamma}^{*}<2.7 \mathrm{GeV}$ requirements.

and $\eta$ and $\eta^{\prime}$ mesons that decay to $\gamma \gamma$. Since the efficiency is known to be strongly dependent on $E_{\gamma}^{*}$ (Section 3.10), and the average multiplicity decreases slowly with $E_{\gamma}^{*}$, the table is partitioned into three energy intervals, to decouple benign energy dependence effects from the real fragmentation problems.

Comparisons of data and simulation performed for the BABAR semi-inclusive analysis [101] can be used to estimate correction factors of approximately $0.395,1.11,1.43$, 1.00 and 0.81 , respectively, for $\mathrm{MC}$ events with $X_{s}$ multiplicities of $2,3,4,5$ and $\geq 6$. Following the lead of Reference [101, these corrections are taken to be independent of hadronic mass or photon energy. When the correction factors are applied as weights to the bins in table 6.4 the overall efficiencies in the three energy intervals shift by $+1.1 \%,+1.0 \%$ and $+0.7 \%$ relative to their original values. In the light of these results, a fractional systematic uncertainty of $1.0 \%$ is assigned to this effect.

Table 6.5 lists the efficiency vs. $n_{\pi^{0}} / n_{\pi^{ \pm}}$, the ratio of the numbers of neutral and charged pions produced from the fragmentation of the $X_{s}$ system. Here no clear trend is observed as a function of increasing ratio, except that the efficiency for states with no neutral pions is a factor of about 1.13 larger than the others. The semiinclusive analysis [101] finds, on average, that the data have relatively more events with $\pi^{0}$ mesons than predicted by the Jetset model. Among the final-state categories studied in that analysis, those with total $X_{s}$ multiplicity $n_{X_{s}} \leq 4$ and $n_{\pi^{0}} \leq 2$ show that about $5 \%$ of the simulated events with $n_{\pi^{0}}=0$ should actually be corrected to 
have $\pi^{0}$ mesons. While information about events with higher $n_{X_{s}}$ or higher $n_{\pi^{0}}$ is not readily available, the effect of transferring $20 \%$ of the events without a $\pi^{0}$ out of that row in Table 6.5 is considered conservative enough to estimate the size of any systematic biases. The result would be a $1.0 \%$ fractional reduction in the overall signal efficiency. This change is taken as a systematic uncertainty.

Combining the two effects in quadrature, a $1.4 \%$ systematic uncertainty is assigned for fragmentation effects.

\subsection{Lepton Tagging}

Deficiencies in the simulation of lepton production, reconstruction or identification have direct impacts both on the selection efficiency of tagging criteria on signal events and on the amount of $B \bar{B}$ background events to be subtracted from data. To account for these deficiencies, various corrections, as briefly mentioned in the earlier chapters, are applied to the Monte Carlo simulation on an event-by-event basis. The details of the corrections and the associated systematic uncertainties are discussed in this section, as organized into the four subsections. The first three subsections describe the corrections and their effects on the signal selection efficiency (i.e. the efficiency of the lepton tagging criteria on signal events):

Subsection 6.3.1 discusses the correction applied to each simulated charged particle to correct particle identification predictions.

Subsection 6.3.2 describes the correction applied to the simulation of $B \rightarrow X_{c} \ell \nu$ decays, which is the largest contributor to the tagging leptons.

Subsection 6.3.3 is a summary of the systematic uncertainty on the signal selection efficiency due to sources of tagging leptons other than $B \rightarrow X_{c} \ell \nu$ decays.

The last part of this section (Subsection 6.3.4) summarizes how the corrections affect the amount of $B \bar{B}$ background to be subtracted and the increase in the uncertainty associated with subtraction. 


\subsubsection{Corrections to Lepton Identification}

As mentioned in Section 3.7.1 the simulation is not satisfactory in predicting particle identification efficiencies and misidentification rates. To remedy this problem, the efficiency and purity of the lepton selection criteria, as measured from independent data control samples, are compiled into look-up tables binned in particles species, charge, momentum and polar angle [61, 63, 92, 93]. The simulated PID prediction for every charged particle in each Monte Carlo event is subsequently overwritten randomly, by using the look-up tables to determine the probability of success. The validity of this method is checked by comparison to earlier measurements of the total tagging efficiency in $B$-mixing data 94 .

The uncertainty in this correction procedure is computed by shifting all entries in the tables coherently by up to \pm 2 standard deviations of the statistical errors of the PID efficiency measurements. Figure 6.4 shows that a shift of one standard deviation results in a $2.2 \%$ change in the lepton tagging efficiency for signal events, which is assigned as the systematic uncertainty.

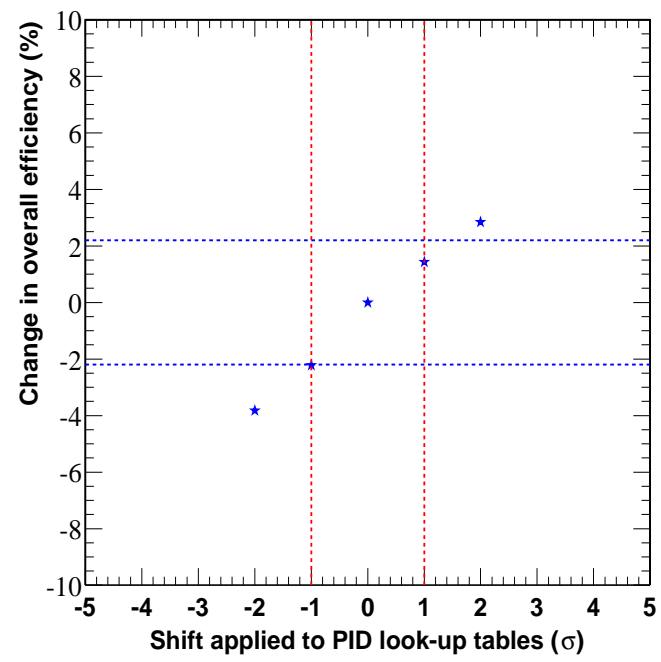

Figure 6.4: The change in selection efficiency for $B \rightarrow X_{s} \gamma$ signal simulation vs. shifts in the particle identification efficiency. The PID efficiencies and misidentification rates are changed in phase by $\pm 1 \sigma$ and $\pm 2 \sigma$ to obtain the points plotted. 


\subsubsection{Corrections on the Semileptonic $B$ Decays with Charm}

Virtually all of the tagging leptons originate from semileptonic $B$ decays, and mainly from $B \rightarrow X_{c} \ell \nu$. Unfortunately, significant discrepancies have been identified between the data and the Monte Carlo generation of these decays [95, 96]. To account for these discrepancies, corrections are applied to the signal and $B \bar{B}$ Monte Carlo events as briefly mentioned in the earlier chapters (Sections 3.10 and 4.7). These corrections are detailed here and related systematic errors on signal selection efficiency are discussed.

The corrections are applied in two steps. The first step is the adjustment of the $B \rightarrow D^{*}$ form factor parameters $\left(R_{1}, R_{2}\right.$ and $\left.\rho^{2}\right)$ for the $B \rightarrow D^{*} \ell \nu$ decay so that they agree with the recently measured values [97. This decay has the largest branching fraction among the $B \rightarrow X_{c} \ell \nu$ decays and contributes the largest fraction of the lepton tags used. The second step is a rescaling of the simulated yields from four contributors to the $X_{c}: D, D^{*}, D^{* *}$ (higher mass resonances) and non-resonant $D^{(*)} \pi$. The rescaling factors are obtained from fits to the inclusive electron spectrum in continuum subtracted data for $p_{e}^{*}>1.1 \mathrm{GeV} / c^{2}$ 95. Table 6.6 lists these rescaling factors along with the default and corrected form factor parameters.

The application of the corrections increases the lepton spectra for c.m.-momenta above $1.1 \mathrm{GeV} / c^{2}$. The enhancement is mainly due to the increase in the $B \rightarrow D \ell \nu$ yields and the form factor adjustments for the $B \rightarrow D^{*} \ell \nu$ component. As a result, the overall efficiency of the tagging criteria on the signal events increases by a factor of $1.079 \pm 0.004$, independent of the energy of the signal photon, $E_{\gamma}^{*}$. Here the uncertainty is only due to the statistics of the flat signal MC samples on which the correction procedure is applied.

In order to compute the systematic uncertainties associated with the corrections, the results of the following studies are added in quadrature:

- The branching-fraction scale factors are varied by one standard deviation of the statistical uncertainties given in Table 6.6 and the results are combined using 


\begin{tabular}{|l|c|c|c|}
\hline \multirow{2}{*}{ Quantity } & \multicolumn{3}{|c|}{ Value } \\
\cline { 2 - 4 } & Default & Adjusted & Alternative factors \\
\hline \hline Form factor ratio $R_{1}$ & 1.18 & $1.328 \pm 0.055 \pm 0.035$ & - \\
Form factor ratio $R_{2}$ & 0.72 & $0.920 \pm 0.044 \pm 0.024$ & - \\
Form factor slope $\rho^{2}$ & 0.90 & $0.769 \pm 0.039 \pm 0.037$ & - \\
\hline $\mathcal{B}\left(D^{*} \ell \nu\right)$ scale factor & 1.00 & $1.00 \pm 0.01$ & 1.16 \\
$\mathcal{B}(D \ell \nu)$ scale factor & 1.00 & $1.42 \pm 0.10$ & 1.08 \\
$\mathcal{B}\left(D^{* *} \ell \nu\right)$ scale factor & 1.00 & $1.58 \pm 0.06$ & 0.94 \\
$\mathcal{B}\left(D^{(*)} \pi \ell \nu\right)$ scale factor & 1.00 & $0.00 \pm 0.04$ & 0.24 \\
\hline
\end{tabular}

Table 6.6: Corrections to the decay parameters for $B \rightarrow X_{c} \ell \nu$ decays [95, 97]. The first three quantities are the $B \rightarrow D^{*}$ form factor parameters. The default values used in the BABAR MC generator are based on an earlier measurement by the CLEO Collaboration [98. The adjusted values are given with statistical and systematic errors. The remaining four quantities are the branching-fraction scale factors relative to what is used in the MC generator. They are given with statistical errors only. The rightmost column gives alternative scale factors derived from an independent measurement that does not apply the form factor adjustments [96].

the observed correlations among the different components [99] :

$$
\begin{aligned}
\rho_{D-D^{*}} & =-0.55, \quad \rho_{D-D^{(*)} \pi}=0.05, \\
\rho_{D-D^{* *}} & =-0.75, \rho_{D^{*}-D^{(*)} \pi}=-0.03 \\
\rho_{D^{*}-D^{* *}} & =0.78, \rho_{D^{(*)} \pi-D^{* *}}=-0.04
\end{aligned},
$$

The observed change in the correction to the signal efficiency is 0.012 .

- The systematic uncertainties on the branching-fraction scale factors are dominated by the MC determination of the selection efficiencies in the inclusive electron spectrum measurement. The technique used to estimate the uncertainty is to repeat the measurement for six alternative sets of selection criteria and compare the results [95]. When the alternative corrections from these additional measurements, which differ by a few percent from those listed in Table 6.6, are applied, the sum-in-quadrature of the observed changes in the correction to the signal efficiency is 0.030 . 
- The form factor uncertainties are given in Table 6.6. The form factor parameters are varied by one standard deviation of the combined statistical and systematic errors and the results are combined using the observed correlations among $R_{1}$, $R_{2}$ and $\rho^{2}$ [97] :

$$
\rho_{R_{1}-R_{2}}=-0.58, \rho_{R_{1}-\rho^{2}}=+0.47 \quad, \quad \rho_{R_{2}-\rho^{2}}=-0.79 .
$$

The observed change in the correction to the signal efficiency is 0.006 .

Combining all these uncertainties gives a total uncertainty of 0.033 on the semileptonic$B$-decay correction of 1.079 . This corresponds to a relative uncertainty of $3.0 \%$ on the signal efficiency.

Finally, a cross check can be performed with the use of an alternative set of correction factors, which are listed in the rightmost column of table 6.6. These alternate corrections have been derived with no adjustments to the $B \rightarrow D^{*}$ form factor parameters [96, so the branching-fraction scale factors are significantly different. The overall correction factor to the signal efficiency with these scale factors is 1.057 , consistent with $1.079 \pm 0.033$.

\subsubsection{Other Sources of Tag Leptons}

Around $5 \%$ of tag leptons arise from processes other than $B \rightarrow X_{c} \ell \nu$. Over $95 \%$ of these come from the following sources: decays of charmed mesons, $J / \psi$ mesons or $\tau$ leptons, charmless semileptonic $B$ decays, and misidentified hadrons. No corrections are made for these processes, but a systematic uncertainty is assigned to the modeling of the rates of production and decay of these particles in the simulation. The relative error on each of the $D^{0}, D^{ \pm}, D_{s}^{+}, J / \psi$ and $\tau$ components is taken as the sum-inquadrature of the fractional error on the inclusive $B$ meson branching fraction to particle being studied (i.e. $\mathcal{B}\left(B \rightarrow D^{0} X\right)$, where $X$ is anything), and the fractional error on inclusive branching fraction of that particle to leptons (i.e. $\mathcal{B}\left(D^{0} \rightarrow \ell^{+} X\right)$ ). These errors on the branching fractions are from the Particle Data Group [4]. For the $B \rightarrow X_{u} \ell \nu$ component, the relative error from an earlier BABAR branching fraction measurement is used [100]. 
The total fractional uncertainty is $8.5 \%$ on this small component of lepton tags, which translates to an uncertainty of $0.4 \%$ on the signal efficiency. The uncertainty related to the modeling of lepton candidates from hadron misidentification is not included in this number, since it is already covered in the PID systematics of Section 6.3 .1 .

\subsubsection{Lepton Corrections on Subtracted $B \bar{B}$ Background}

The corrections and uncertainties described in the first three parts of this section are applied to the $B \bar{B}$ simulation as well. Among the three, only the $B \rightarrow X_{c} \ell \nu$ corrections have significant effects on the extraction of the $B \rightarrow X_{s} \gamma$ signal, since the discrepancies related to the other two are absorbed into the computation of the $\pi^{0} / \eta$ correction factors of Section 4.2 and the diluted uncertainties are very small compared to the uncertainties on the various $B \bar{B}$ corrections.

The semileptonic- $B$-decay corrections are applied to the $B \bar{B}$ Monte Carlo simulation the same way they are applied to the signal simulation. The $B \rightarrow D^{*}$ form factor parameters are adjusted and the events are reweighted using the branchingfraction scale factors. The only difference is the application of the corrections to the electron component of the $B \bar{B}$ background twice: Once for the $B$-meson that yields the background electron and another time for the $B$-meson that yields the tag lepton. The overall correction on the $B \bar{B}$ background is 1.077 , consistent with what has been observed for the signal MC events. (The corrections in bins of $E_{\gamma}^{*}$ are listed under $\alpha_{\text {semi }}$ column in Table 4.22.)

However, a further adjustment is necessary for the $B \bar{B}$ events. The $\pi^{0} / \eta$ correction factors (Section 4.2) absorb some of the semileptonic- $B$-decay corrections as well, since they have been computed without the semileptonic corrections, i.e. the lack of corrections to the lepton tags reduces the normalization of $B \bar{B}$ events, thus increasing the $\pi^{0} / \eta$ correction. Therefore if both corrections are applied simultaneously, the $B \bar{B}$ background will be overcorrected. On the other hand, simply not applying the semileptonic corrections to the $\pi^{0} / \eta$ component of the $B \bar{B}$ background is not a viable option, since the lepton tag requirements that are used in the selection of $\pi^{0}(\eta)$ 
samples (Section 4.2.1) are different from the regular analysis: the minimum electron (muon) c.m.-momentum requirement has been lowered to $1.0(1.1) \mathrm{GeV} / c$ for the $\pi^{0} / \eta$ analysis. At these lower momenta the magnitude of the semileptonic correction is much smaller than the average value of 1.077 mentioned above, mainly as a consequence of the zero weight given to the non-resonant, $D^{(*)} \pi$, component, which has a lepton spectrum peaked at lower values of $p_{l}^{*}$.

To resolve this situation, it is essential to determine the contribution of the semileptonic- $B$-decay discrepancy in the computed value of the $\pi^{0} / \eta$ corrections. A simple way of doing this is to apply the semileptonic corrections to the $B \bar{B}$ MC samples used in the $\pi^{0} / \eta$ fits and measure how much they affect the normalization of the samples. This yields semileptonic correction factors of $1.025 \pm 0.001$ and $1.029 \pm 0.001$ for $\pi^{0}$ and $\eta$ samples, respectively, where the error is statistical. The systematic error on these factors is 0.033 , which is derived in an identical manner to the uncertainty on the signal efficiency from this source, as described earlier in Subsection 6.3.2. The semileptonic corrections have no significant dependence on $m_{\gamma \gamma}$ or $E_{\pi^{0} / \eta}^{*}$. Therefore, all $\pi^{0}(\eta)$ corrections should be reduced by a factor of $1 / 1.025(1 / 1.029)$ to account for neglecting the semileptonic corrections in their derivation. This scaling of the $\pi^{0} / \eta$ corrections is done when they are applied to the $B \bar{B} \mathrm{MC}$ events (Section 4.7); that is, the results quoted in Section 4.2.4 do not include this additional factor, but the values in Table 4.22 do.

Finally, it is important to realize that the uncertainty in the semileptonic corrections for the $B \bar{B}$ background and the signal are fully correlated. The effects of this correlation will be discussed in Section 6.5.

\subsection{Data Sample Normalization}

There are several small systematic effects associated with the determination of the sample sizes listed in Table 2.1. First, the on-resonance data has an uncertainty of $1.1 \%$ on the total number of $B \bar{B}$ events. Second, the integrated luminosity of the off-resonance data has a $0.6 \%$-uncertainty relative to the integrated luminosity of the on-resonance data; however, given the relative amount of off-resonance data to 
expected signal in any relevant $E_{\gamma}^{*}$ range, this translates to an effect of order $0.3 \%$ on signal. Added in quadrature, the net uncertainty is $1.1 \%$.

\subsection{Simulation Based $B \bar{B}$ Subtraction}

The systematic uncertainty on the $B \bar{B}$ background is taken to be the uncertainty on the overall correction factor applied to the $B \bar{B}$ Monte Carlo sample, in quadrature with the uncertainty due to limited $B \bar{B}$ Monte Carlo statistics. Taking the correlations between the uncertainties among various bins (Table 4.23) into account, the uncertainty on the number of $B \bar{B}$ background events has been computed as listed in Table 4.21. As an example, in the $E_{\gamma}^{*}$ range of 2.0 to $2.7 \mathrm{GeV}$, an uncertainty of $7.9 \%$ can be read from this table. Table 6.7 shows the breakdown of this uncertainty into its sources.

\begin{tabular}{|l|c|}
\hline Component & Systematic (\%) \\
\hline$\pi^{0}$ & 6.6 \\
$\eta$ & 2.6 \\
$\omega$ & 0.5 \\
$\eta^{\prime}$ & 0.9 \\
$\bar{n} / \bar{p}$ & 0.1 \\
$e^{ \pm}$ & 0.4 \\
$\pi^{0}(\eta)$ veto & 1.7 \\
$B \rightarrow X_{c} \ell \nu$ & 1.0 \\
Light-quark FSR & 0.04 \\
MC statistics & 2.8 \\
\hline Total & 7.9 \\
\hline
\end{tabular}

Table 6.7: The systematic uncertainties on the $B \bar{B}$ background in the range $2.0<E_{\gamma}^{*}<2.7 \mathrm{GeV}$, expressed as a percentage of the $B \bar{B}$ yield.

Obviously the fractional effect of this uncertainty on the extracted branching fraction is $\Delta \mathrm{B} / \mathrm{S}$, where $\mathrm{S}$ is the signal yield after the subtraction, and $\mathrm{B} \pm \Delta \mathrm{B}$ is the number of $B \bar{B}$ background events subtracted.

There is, however, one small complication. As briefly mentioned at the end of 
Section 6.3.4, the uncertainties in the semileptonic- $B$-decay corrections to the signal and $B \bar{B}$ backgrounds are correlated. In fact, they reinforce: if the semi-leptonic correction increases, the number of $B \bar{B}$ events, to be subtracted increases, so the signal yield decreases. At the same time, a larger correction implies a larger computed signal selection efficiency, and that would result in a smaller value for the branching fraction. Therefore these contributions to the overall systematic error must be added linearly. Given the systematic uncertainty of 3.0\% for signal (Section 6.3.2) and $1.02 \%$ for $B \bar{B}$ (Table 6.7), the net uncertainty on the measured branching fraction due to semileptonic- $B$-decay corrections is $(3.0+1.02 \mathrm{~B} / \mathrm{S}) \%$. Since the first term is already accounted for in Section 6.3.2, it is convenient to increase the $B \bar{B}$ background error such that when added in quadrature with to the $3.0 \%$ uncertainty related to the signal, the total error still remains the same. The total $B \bar{B}$ uncertainty given in Table 6.1 reflects this additional term, therefore the rows on that table can simply be added in quadrature to obtain the total systematic uncertainty.

\subsection{Signal Monte Carlo Simulation}

While the arbitrariness in the assumed signal model in the extraction of the total branching fraction introduces model-dependence uncertainties, these will be addressed in the next chapter. The uncertainties discussed in this section relate to the effects after a signal model has been been selected.

The first such effect is simply the systematic uncertainty introduced due to the statistics of the available signal Monte Carlo samples. With the larger flat Monte Carlo used in the computation of the signal efficiencies, this systematic error is about $1.1 \%$.

The signal model, as described in Section 1.8, is composed of a mixture of $B \rightarrow$ $K^{*} \gamma$ and $B \rightarrow X_{s} \gamma$ with a sharp boundary at $m_{\text {cutoff }}=1.1 \mathrm{GeV} / c^{2}$. In reality this boundary is more of a smooth transition. To take this into account, an uncertainty is computed by applying the Kagan and Neubert prescription with the mass cutoff varied between 1.0 and $1.2 \mathrm{GeV} / c^{2}$. The result for the 2.0 to $2.7 \mathrm{GeV} E_{\gamma}^{*}$ range is an efficiency change (from the default $m_{\text {cutoff }}$ of $1.1 \mathrm{GeV}$ ) of $+0.14 \% /-0.28 \%$. The results 
for the other energy ranges are consistent with these values to within $0.01 \%$. It is not surprising that these systematic variations are small, because such a variation is sensitive only to the difference in the selection efficiency for the $K^{*} \gamma$ component and the highest- $E_{\gamma}^{*}$ region of the $X_{s}$ component, and that difference is small. 


\section{Chapter 7}

\section{Results}

The determination of the inclusive branching fraction for $B \rightarrow X_{s} \gamma$ and the HQE parameters is the last step of the analysis. This step offers new challenges because of various effects that are dependent on the signal model. In particular, the selection efficiency in bins of photon energy shows a significant model-dependence, which requires a special bootstrapping method to reduce. The selection efficiencies are needed to extract the moments of the photon energy spectrum, which are used to determine which signal model describes the data best; and a model is necessary to determine the selection efficiencies. This circular dependency is described and resolved in Section [7.1. The other important challenge is the translation of the moments from the c.m. frame to the rest frame of the $B$-meson. This is discussed in Section [7.3. Finally, fits are performed to the measured quantities and HQE parameters are extracted (Section $[7.5)$.

\subsection{Model Dependence of Selection Efficiency}

The signal selection efficiency varies as a function of the energy of the signal photon as discussed in Section 3.10. Since different signal models have different predictions for the photon energy spectrum, the overall efficiency for the integrated signal range of 1.9 to $2.7 \mathrm{GeV}$ is expected to be dependent on the assumed model. To reduce this dependence, the extraction of the total signal could be carried out in $100 \mathrm{MeV}$ bins of 
$E_{\gamma}^{*}$ : the variation of the energy distribution within each bin is simply much smaller than the variation in the whole energy spectrum.

However, this simple approach is not as effective as it seems to be. Table 7.1 gives the signal efficiency in bins of $E_{\gamma}^{*}$ for a few different signal models, normalized to the efficiency for the default (KN480) model. The model dependence of the efficiency in low-energy bins is comparable to the model dependence in the integrated $E_{\gamma}^{*}$ range.

\begin{tabular}{|l|c|c|c|c|c|c|c|c|c|}
\hline$E_{\gamma}^{*}$ Bin & 1.9 & 2.0 & 2.1 & 2.2 & 2.3 & 2.4 & 2.5 & 2.6 & sig \\
\hline KN465 & 0.927 & 0.913 & 0.951 & 0.953 & 0.971 & 0.973 & 0.992 & 0.993 & 0.909 \\
\hline LNP05 & 0.930 & 0.931 & 0.964 & 0.957 & 0.965 & 0.953 & 0.985 & 0.983 & 0.904 \\
\hline BBU03 & 0.949 & 0.953 & 0.978 & 0.964 & 0.966 & 0.952 & 0.983 & 0.980 & 0.917 \\
\hline
\end{tabular}

Table 7.1: The ratios of the overall signal efficiencies for a few alternative signal models to that for the default (KN480) model in bins of reconstructed $E_{\gamma}^{*}$. The bins are labeled by their low edges in $\mathrm{GeV}$; the sig column corresponds to the $1.9<E_{\gamma}^{*}<2.7 \mathrm{GeV}$ range.

The reason for this large variation in $100 \mathrm{MeV}$ bins is subtle. Each bin of reconstructed $E_{\gamma}^{*}$ is populated by photons of varying true $E_{\gamma}^{*}$. As an example, one can consider a low-lying bin in reconstructed $E_{\gamma}^{*}$ like $2.0-2.1 \mathrm{GeV}$. Since the spectrum is strongly rising with energy in this region, a disproportionate number of the events in this bin are due to feeddown from higher values of true $E_{\gamma}^{*}$ mismeasured because of the calorimeter energy resolution. Much of the dependence of the selection efficiency on $E_{\gamma}^{*}$ (Figure [3.13) is due to effects related to the true value of the photon energy rather than to its reconstructed value. Thus the selection efficiency for the feeddown events in the 2.0-to- $2.1 \mathrm{GeV}$ bin is higher than the efficiency of the native events in this bin. The average energy shift (from true to reconstructed energy) is dependent on the underlying spectral shape, so different signal models will have different fractions of high-true- $E_{\gamma}^{*}$ photons populating any given reconstructed- $E_{\gamma}^{*}$ bin. This is demonstrated in Table 7.2 which shows the mean value of the true $E_{\gamma}^{*}$ in bins of the reconstructed $E_{\gamma}^{*}$.

Unfortunately, the model uncertainty that would be introduced by the selection of an arbitrary model is very large: the single-bin efficiency $(1.9-2.7 \mathrm{GeV})$ shows almost 30\% variation among the various models considered for systematic studies 


\begin{tabular}{|c|c|c|c|c|c|c|c|c|c|}
\hline Reco. $E_{\gamma}^{*}$ Bin & 1.85 & 1.95 & 2.05 & 2.15 & 2.25 & 2.35 & 2.45 & 2.55 & 2.65 \\
\hline$\left\langle E_{\text {true }}^{*}\right\rangle_{\mathrm{KN} 465}$ & 1.962 & 2.039 & 2.123 & 2.208 & 2.292 & 2.377 & 2.460 & 2.543 & 2.620 \\
$\left\langle E_{\text {true }}^{*}\right\rangle_{\mathrm{KN} 480}$ & 2.014 & 2.076 & 2.148 & 2.225 & 2.304 & 2.385 & 2.465 & 2.547 & 2.624 \\
\hline
\end{tabular}

Table 7.2: The mean value of true $E_{\gamma}^{*}(\mathrm{in} \mathrm{GeV})$ in bins of reconstructed $E_{\gamma}^{*}$ for the KN465 and KN480 models, right after the skim selection criteria are applied. The bins are labeled by their central energies in $\mathrm{GeV}$.

(Appendix $\mathrm{A}$ ). Apart from the fact that such a large uncertainty would defy the logic of the whole precision measurement, there appears to be the more serious problem: the arbitrariness of which model to choose to extract the central value for the measurement.

The solution to this problem is to use the data itself to choose the proper model, i.e. a bootstrapping method. In order to do this, a quantity is needed that can be extracted from the data with little dependence on the assumed selection efficiency, but itself contains information that can be used to determine the proper value of the selection efficiency. Since the bin-by-bin efficiency is almost a linear function of energy (Figure 3.12), one such quantity is the mean value of the photon energy.

Table 7.3 shows the truncated mean value of the reconstructed- $E_{\gamma}^{*}$ distribution in the range 2.0 to $2.7 \mathrm{GeV}$ for the four different parameterizations of the $\mathrm{KN}$ model used for systematic studies. Also tabulated are the single-bin efficiency values computed using each model for three different choices of $E_{\gamma}^{*}$ range. In Figure 7.1 the 2.0-to-2.7 GeV single-bin efficiency is plotted as a function of the truncated mean energy for these four parameterizations along with the models detailed in Appendix $\AA$. The relationship between the single-bin efficiency and the mean energy is remarkably linear, regardless of the underlying signal model used, or the particular parameterization of a given model. (The deviation from linearity is less than $0.5 \%$ for the 2.0-to- $2.7 \mathrm{GeV}$ range and less than $2.0 \%$ for the 1.9 -to- $2.7 \mathrm{GeV}$ range. This is added as a tiny additional systematic uncertainty on the final figures.)

The problem of determining the most appropriate efficiency value is therefore translated into the measurement of the mean energy and bootstrapping. Using the efficiency figures for the KN465 model, the truncated $E_{\gamma}^{*}$ mean for data in the 


\begin{tabular}{|l|c|c|c|c|}
\hline & Mean $E_{\gamma}^{*}$ & \multicolumn{3}{|c|}{ Efficiency $(\%)$ for $E_{\gamma}^{*}$ range } \\
\cline { 3 - 5 } Model & $(\mathrm{GeV})$ & $1.9-2.7 \mathrm{GeV}$ & $2.0-2.7 \mathrm{GeV}$ & $2.1-2.7 \mathrm{GeV}$ \\
\hline KN455 & 2.3033 & 1.578 & 1.657 & 1.753 \\
KN460 & 2.3112 & 1.618 & 1.691 & 1.782 \\
KN465 & 2.3202 & 1.664 & 1.731 & 1.814 \\
KN480 & 2.3531 & 1.830 & 1.876 & 1.935 \\
\hline
\end{tabular}

Table 7.3: Predictions for the truncated mean value of the reconstructed photon energy, $\left\langle E_{\gamma}^{*}\right\rangle$ and the overall selection efficiency (including acceptance) for the four parameterizations of the Kagan-Neubert (KN) model. The mean energies are presented for the $E_{\gamma}^{*}$ range of 2.0 to $2.7 \mathrm{GeV}$, whereas the efficiencies are given for three different energy ranges.

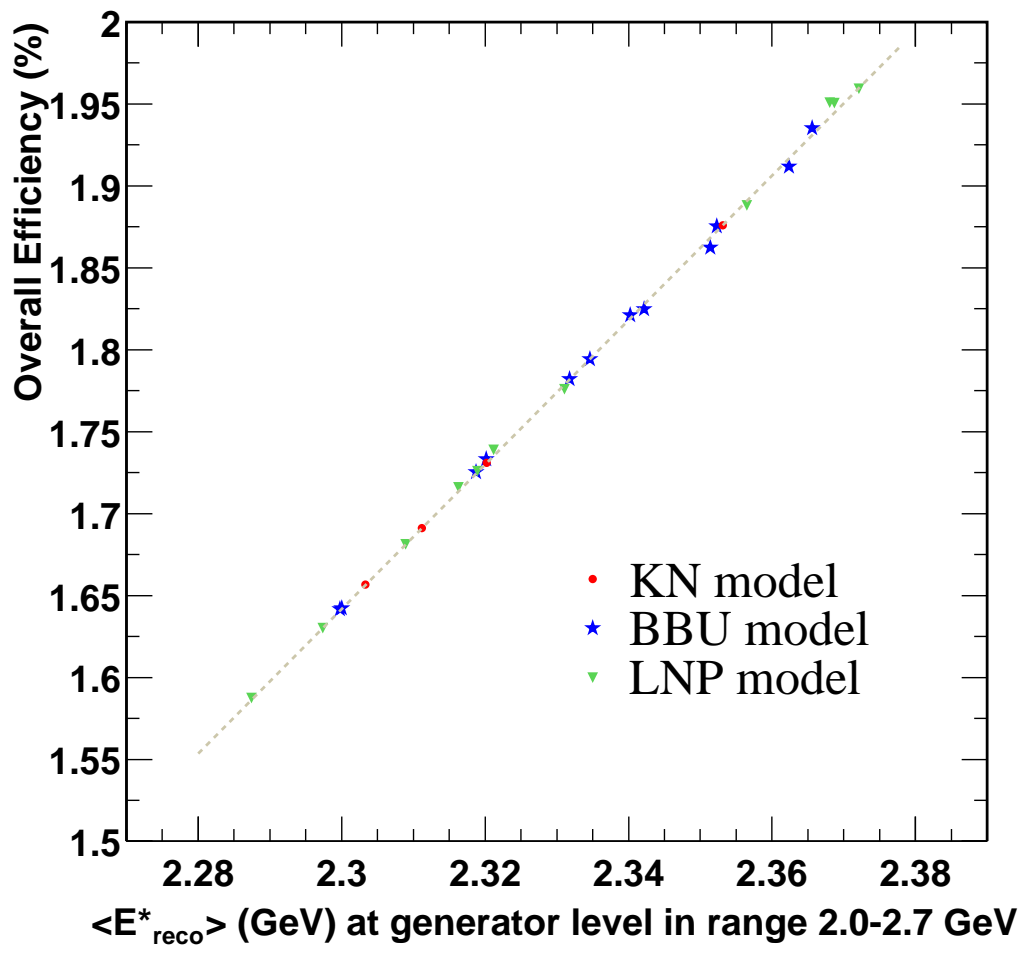

Figure 7.1: Single-bin signal efficiency vs. mean reconstructed photon energy, $\left\langle E_{\gamma}^{*}\right\rangle$, for $2.0<E_{\gamma}^{*}<2.7 \mathrm{GeV}$, from Monte Carlo simulation. Shown are points for the four KN models listed in Table 7.3 and for the BBU and LNP model parameterizations given in Appendix A 
2.0-to- $2.7 \mathrm{GeV}$ range is measured to be $(2.3045 \pm 0.0158 \pm 0.0083 \pm 0.0062) \mathrm{GeV}$, where the first error is statistical, the second is due to $B \bar{B}$ subtraction systematics, and the third due to photon energy scale systematics. Given the $\pm 0.5 \%$ uncertainty assigned in Section 6.1.1, the value of $6.2 \mathrm{MeV}$ might appear small for the photon energy scale systematic. However, since the mean is measured in a truncated range, the effect is subdued. Other systematic effects associated with photon energy measurement (Table 6.2) are largely independent of $E_{\gamma}^{*}$, and hence do not affect the mean.

Comparison with the signal models in Table 7.3 indicates that the central value of $2.3045 \mathrm{GeV}$ falls between the mean values for KN455 and KN460 models. If the KN460 or KN455 efficiency figures are used instead of KN465, the mean value for the data is a few $\mathrm{MeV}$ lower, 2.3035 or $2.3027 \mathrm{GeV}$, respectively. The KN460 efficiency figures are chosen to extract the partial branching fractions and the above-mentioned uncertainties in the mean measurement, which quadratically add up to $18.9 \mathrm{MeV}$, are translated into the uncertainty in the branching fraction, using the linear relation in Figure 7.1 .

Finally, there is a small additional uncertainty on the measured mean that needs to be addressed. The bootstrapping method relies on the assumption that this quantity can be measured without much dependence on the particular model used in its computation. However, as mentioned in the last paragraph, using different $\mathrm{KN}$ parameterizations change the measured data mean by a few $\mathrm{MeV}$. To assess the uncertainty associated with this, among all the models shown on Figure 7.1, the ones that predict a mean value closer to the nominal (KN460) value than its measurement uncertainty are identified. There are thirteen such models (KN455, KN465, five parameterizations of the $\mathrm{BBU}$ and six of the LNP models), which predict a mean within 18.9 MeV of $2.3035 \mathrm{GeV}$. When the efficiency figures from these thirteen models are used instead of the KN460 efficiencies, the measured data mean varies by ${ }_{-3.3}^{+6.8} \mathrm{MeV}$. To account for this small variation, $6.8 \mathrm{MeV}$ is added in quadrature to $18.9 \mathrm{MeV}$. 


\subsection{Partial Branching Fractions in $\Upsilon(4 S)$ Frame}

Given the event yields and the selection efficiency, the partial branching fractions in a particular energy range, $\Delta E_{\gamma}$, can be computed as:

$$
\mathcal{B}\left(B \rightarrow X \gamma, E_{\gamma} \text { in } \Delta E_{\gamma}\right)=\frac{\text { Number of events in } \Delta E_{\gamma}}{2 n_{B \bar{B}} \times \epsilon_{\mathrm{KN} 460}^{\text {in } \Delta E_{\gamma}}}
$$

Table 7.4 lists the partial branching fractions in four overlapping ranges of reconstructed $E_{\gamma}^{*}$, using the single-bin efficiencies computed for those ranges using the KN460 model. The systematic uncertainty on the listed numbers include all the effects discussed in Chapter [6] and the model-dependent-efficiency errors refer to the effects mentioned in the previous section.

\begin{tabular}{|c|c|c|}
\hline$E_{\gamma}^{*}(\mathrm{GeV})$ & Partial Branching Fraction & Mean $E_{\gamma}^{*}(\mathrm{GeV})$ \\
\hline $1.9-2.7$ & $3.64 \pm 0.29 \pm 0.33 \pm 0.24$ & $2.2696 \pm 0.0253 \pm 0.0151 \pm 0.0067 \pm 0.0070$ \\
$2.0-2.7$ & $3.32 \pm 0.26 \pm 0.28 \pm 0.17$ & $2.3035 \pm 0.0155 \pm 0.0083 \pm 0.0060 \pm 0.0068$ \\
$2.1-2.7$ & $2.84 \pm 0.23 \pm 0.23 \pm 0.12$ & $2.3501 \pm 0.0135 \pm 0.0050 \pm 0.0054 \pm 0.0043$ \\
$2.2-2.7$ & $2.23 \pm 0.19 \pm 0.18 \pm 0.06$ & $2.4116 \pm 0.0121 \pm 0.0029 \pm 0.0044 \pm 0.0021$ \\
\hline
\end{tabular}

Table 7.4: Partial branching fractions for $B \rightarrow X \gamma$ (with statistical, systematic and model-dependent efficiency errors) and truncated means (with statistical, BB systematic, energy scale systematic and model-dependent efficiency errors) in four different reconstructed- $E_{\gamma}^{*}$ ranges.

Also tabulated in the same table are the truncated mean $E_{\gamma}^{*}$ values for each range. Taking into account the correlations according to Table 4.23, the statistical and systematic uncertainties are computed from the uncertainties on the efficiency-corrected signal yields $N_{i}$, where $i$ labels the energy bin with midpoint $E_{i}$, using:

$$
\frac{\partial\left\langle E_{\gamma}^{*}\right\rangle}{\partial N_{i}}=\frac{E_{i}-\left\langle E_{\gamma}^{*}\right\rangle}{\Sigma N_{i}}
$$

Figure 7.2 shows the $E_{\gamma}^{*}$ spectrum obtained by correcting the raw spectrum by KN460 bin-by-bin efficiencies. The model-dependence uncertainties on the efficiencies 
in individual bins are not constrained by the bootstrapping technique, so a conservative approach is taken: the uncertainty assigned to a given bin is equal to the maximum efficiency deviation observed for that bin among all the models considered for systematic studies (Table 1.1 and Appendix (A). These uncertainties, the other efficiency systematics, and parts of the $B \bar{B}$ background systematics are highly correlated among the bins.

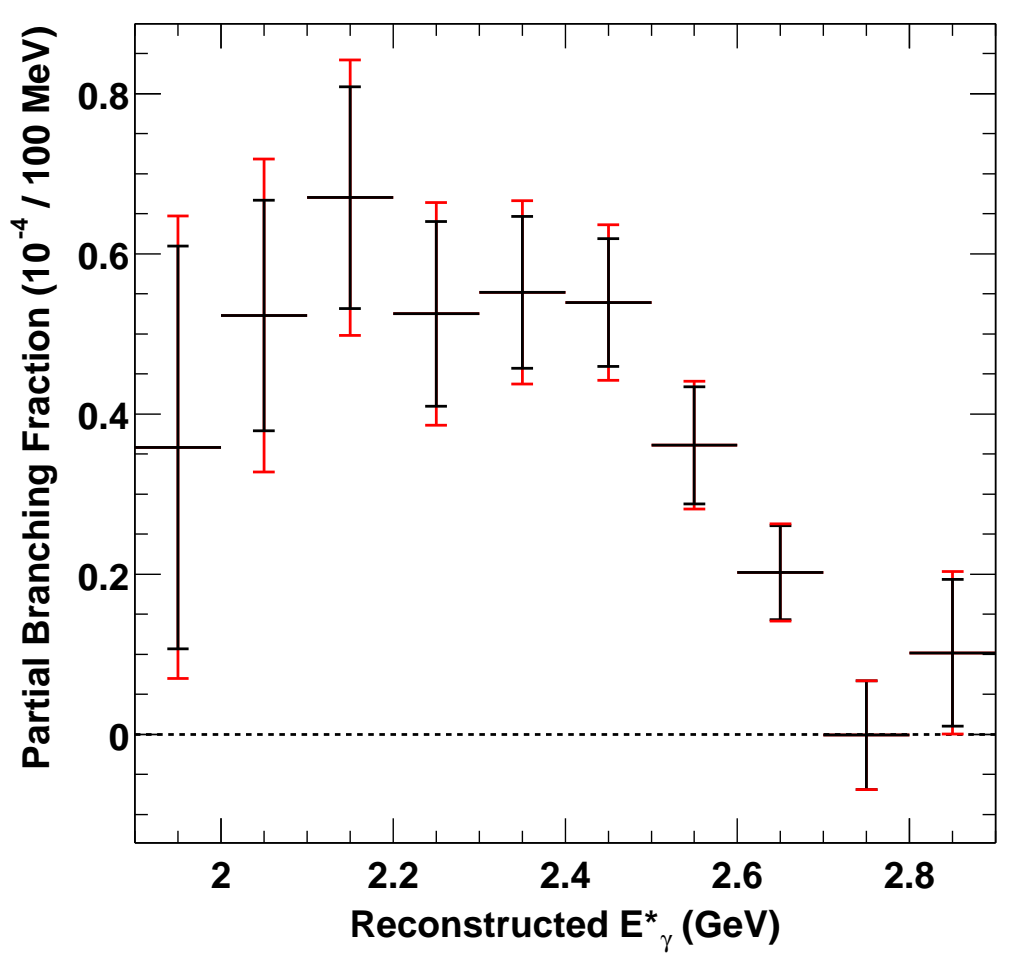

Figure 7.2: Efficiency-corrected reconstructed- $E_{\gamma}^{*}$ distribution, in the originally-blinded range of $E_{\gamma}^{*}$. The inner error bars are statistical only. The outer error bars include systematic and model-dependence uncertainties in quadrature. 


\subsection{Truncated Moments in the $B$-Meson Rest Frame}

Since the theoretical calculations for the moments of photon energy spectrum are performed in the rest frame of the $B$-meson, the moments of the measured $E_{\gamma}^{*}$ spectrum need to be translated. This translation must account for the following three effects:

- Doppler shift due to the motion of the $B$ meson in the $\Upsilon(4 S)$ frame.

- Calorimeter smearing due to EMC resolution.

- Smearing at the energy cutoffs. The minimum reconstructed energy requirement in the the c.m. frame corresponds to a gradual turning-on for the true $E_{\gamma}$, and not a sharp cutoff.

Each of the three effects can be taken into account by a simple additive correction. However, despite its simplicity, such a correction is mathematically well motivated. If the energy spectrum in the $B$-meson rest frame is given by a function $f_{t h}\left(E^{\prime}\right)$ and the smearing effects can be represented by resolution function $R\left(E-E^{\prime}\right)$, then the smeared spectrum $f_{\text {reco }}(E)$ can be expressed as a convolution integral, $f_{\text {reco }}=f_{\text {th }} \otimes R$. A well known property of the convolution operation is that the first moment of the resulting function is the sum of the first moments of the input functions [102. The same statement also applies to the centralized second moments, $\left\langle(E-\langle E\rangle)^{2}\right\rangle$, which will be referred to simply as the second moments.

Therefore the moments of the measured spectrum are related to the moments of the true spectrum by a correction given by the moments of the resolution function, which can easily be determined from the signal Monte Carlo simulation. Despite being measured in binned distributions rather than continuous functions, these determined corrections provide excellent predictions in Monte Carlo tests.

The last effect to take into account is the application of the requirements on the reconstructed $E_{\gamma}^{*}$, while the theoretical computations are for similar cutoffs on true $E_{\gamma}$ in the $B$-meson rest frame. In order to estimate the size of this effect, the measured $E_{\gamma}^{*}$ truncated moments, after they have been corrected using the moments of the resolution function, are compared to the true- $E_{\gamma}$ truncated moments. Table 7.5 gives an example of this procedure. 


\begin{tabular}{|c|c|c|}
\hline Quantity & Requirement & Value $(\mathrm{GeV})$ \\
\hline$\left\langle\operatorname{Reco} . E_{\gamma}^{*}\right\rangle$ & $1.9<$ Reco. $E_{\gamma}^{*}<2.7 \mathrm{GeV}$ & 2.311 \\
\hline$-\left\langle R_{\text {Doppler }+ \text { EMC }}\right\rangle$ & $1.9<$ Reco. $E_{\gamma}^{*}<2.7 \mathrm{GeV}$ & 0.018 \\
\hline Predicted $\left\langle E_{\gamma}\right\rangle$ & $1.9<$ Reco. $E_{\gamma}^{*}<2.7 \mathrm{GeV}$ & 2.329 \\
\hline Actual $\left\langle E_{\gamma}\right\rangle$ & True $E_{\gamma}>1.9 \mathrm{GeV}$ & 2.323 \\
\hline$\Delta_{\text {cut }}$ correction & & -0.006 \\
\hline
\end{tabular}

Table 7.5: Computation of $\Delta_{\text {cut }}$ correction to the measured 1.9-2.7 GeV moment, using the KN460 signal simulation. The truncated first moment of the reconstructed photon energy $\left(E_{\gamma}^{*}\right)$ spectrum in the c.m. frame, in the range $1.9<E_{\gamma}^{*}<2.7 \mathrm{GeV}$, is initially corrected for the Doppler and EMC smearing effects (the negative of the sum of first moments of the Doppler and EMC resolution functions, also computed with the $1.9<E_{\gamma}^{*}<2.7 \mathrm{GeV}$ requirement applied), and then compared to the first moment of the true photon energy $\left(E_{\gamma}\right)$ distribution in the $B$-meson rest frame, with a requirement of true $E_{\gamma}>1.9 \mathrm{GeV}$.

It is essential to note that these corrections are model-dependent. The resolution function has some dependence on the energy (Figure 2.71) and thus its moments depend weakly on the signal Monte Carlo spectrum used in its extraction. The energy-cutoff correction, $\Delta_{c u t}$, also depends on the shape of the assumed spectrum. In order to account for this dependence, the total correction, $\Delta_{\Upsilon \rightarrow B}$, is computed using the KN and BBU models considered for systematic studies (Table 1.1 and Appendix A.1), and the average of the maximum and minimum is taken as the central value of the correction, with an error equal to half of the difference between the two extrema. Table 17.6 gives these corrections and the final corrected truncated first moments in the $B$-meson rest frame.

The measured and corrected second moments of the photon energy distribution are given in Table [7.7. As for the first moments, the measurement is initially performed for the reconstructed $E_{\gamma}^{*}$ spectrum, with values computed by assigning all events in a $100-\mathrm{MeV}$ bin to the center of that bin. The uncertainties on these measured values are computed from the uncertainties on the efficiency-corrected signal yields $N_{i}$, where $i$ 


\begin{tabular}{|c|c|c||c|}
\hline $\begin{array}{c}E_{\min } \\
(\mathrm{GeV})\end{array}$ & $\begin{array}{c}\left\langle E_{\gamma}^{*}\right\rangle \pm \text { stat } \pm \text { sys } \pm \text { model } \\
(\mathrm{GeV}) \text { for } E_{\min }<E_{\gamma}^{*}<2.7 \mathrm{GeV}\end{array}$ & $\begin{array}{c}\Delta_{\Upsilon \rightarrow B} \pm \text { model } \\
(\mathrm{GeV})\end{array}$ & $\begin{array}{c}\left\langle E_{\gamma}\right\rangle \pm \text { stat } \pm \text { sys } \pm \text { model } \\
(\mathrm{GeV}) \text { for } E_{\min }<E_{\gamma}\end{array}$ \\
\hline 1.9 & $2.270 \pm 0.025 \pm 0.017 \pm 0.005$ & $0.018 \pm 0.007$ & $2.288 \pm 0.025 \pm 0.017 \pm 0.012$ \\
2.0 & $2.304 \pm 0.016 \pm 0.010 \pm 0.005$ & $0.012 \pm 0.007$ & $2.316 \pm 0.016 \pm 0.010 \pm 0.012$ \\
2.1 & $2.350 \pm 0.014 \pm 0.007 \pm 0.003$ & $0.005 \pm 0.007$ & $2.355 \pm 0.014 \pm 0.007 \pm 0.010$ \\
2.2 & $2.412 \pm 0.012 \pm 0.005 \pm 0.002$ & $-0.005 \pm 0.006$ & $2.407 \pm 0.012 \pm 0.005 \pm 0.008$ \\
\hline
\end{tabular}

Table 7.6: The measured first moments of the photon spectrum in various overlapping energy ranges, labeled by the minimum energy, $E_{\min }$. The moments are given both for the reconstructed $E_{\gamma}^{*}$ (as in Table 7.4) and for the corrected $E_{\gamma}$ in the $B$-meson rest frame. Also listed are the correction factors, $\Delta_{\Upsilon \rightarrow B}$, that are used to translate between the two quantities. The model-dependence errors on $\left\langle E_{\gamma}^{*}\right\rangle$ are correlated with those on $\Delta_{Y \rightarrow B}$, so these are added linearly.

labels the energy bin with midpoint $E_{i}$, taking

$$
\frac{\partial \operatorname{var}\left(E_{\gamma}^{*}\right)}{\partial N_{i}}=\frac{\left(E_{i}-\left\langle E_{\gamma}^{*}\right\rangle\right)^{2}-\operatorname{var}\left(E_{\gamma}^{*}\right)}{\Sigma N_{i}},
$$

if the bin $i$ is in the truncated range, or zero otherwise. The systematic uncertainties in the measured values arise entirely from the $B \bar{B}$ background subtraction, with binto-bin correlations taken into account using Table 4.23. The $E_{\gamma}^{*}$ second moments have been computed with the same variation of efficiency models used for the first moments; this variation is less than $0.0002 \mathrm{GeV}^{2}$, which is ignored.

\begin{tabular}{|c|c|c||c|}
\hline $\begin{array}{c}E_{\min } \\
(\mathrm{GeV})\end{array}$ & $\begin{array}{c}\text { var }\left(E_{\gamma}^{*}\right) \pm \text { stat } \pm \text { sys }\left(\mathrm{GeV}^{2}\right) \\
\text { for } E_{\min }<E_{\gamma}^{*}<2.7 \mathrm{GeV}\end{array}$ & $\begin{array}{c}\Delta_{\Upsilon \rightarrow B} \pm \text { sys } \pm \text { model } \\
\left(\mathrm{GeV}^{2}\right)\end{array}$ & $\begin{array}{c}\operatorname{var}\left(E_{\gamma}\right) \pm \text { stat } \pm \text { sys } \pm \text { model } \\
\left(\mathrm{GeV}^{2}\right) \text { for } E_{\min }<E_{\gamma}\end{array}$ \\
\hline 1.9 & $0.0403 \pm 0.0049 \pm 0.0022$ & $-0.0075 \pm 0.0005 \pm 0.0025$ & $0.0328 \pm 0.0049 \pm 0.0023 \pm 0.0025$ \\
2.0 & $0.0326 \pm 0.0026 \pm 0.0009$ & $-0.0060 \pm 0.0004 \pm 0.0020$ & $0.0266 \pm 0.0026 \pm 0.0010 \pm 0.0020$ \\
2.1 & $0.0246 \pm 0.0019 \pm 0.0005$ & $-0.0055 \pm 0.0003 \pm 0.0015$ & $0.0191 \pm 0.0019 \pm 0.0006 \pm 0.0015$ \\
2.2 & $0.0161 \pm 0.0014 \pm 0.0003$ & $-0.0045 \pm 0.0002 \pm 0.0005$ & $0.0116 \pm 0.0014 \pm 0.0004 \pm 0.0005$ \\
\hline
\end{tabular}

Table 7.7: The measured second moments (variances) of the photon spectrum in various energy ranges, labeled by the minimum energy, $E_{\min }$. The variances are given both for the reconstructed $E_{\gamma}^{*}$ and for the corrected $E_{\gamma}$ in the $B$-meson rest frame. Also listed are the correction factors, $\Delta_{\Upsilon \rightarrow B}$, that are used to translate between the two quantities. 
Using the same technique described for the first moments, $\Delta_{\Upsilon \rightarrow B}$ corrections and their model-dependence errors are computed also for the second moments. However, an additional systematic uncertainty, found to be negligible for the first moments, is assigned to account for the observed data-simulation differences in EMC resolution (Section 6.1.1). The Monte Carlo representation of the calorimeter resolution is replaced by a Novosibirsk function, whose tail parameters are varied according to the fits to the VCS data and Monte Carlo samples. The resulting changes in the corrections are taken as the systematic uncertainty (Table 7.7).

Finally, for both the first and second moments, the effect of using 50-MeVbins rather than 100-MeVbins have been assessed. While the central values change slightly, the effect is more-than-three times smaller than the statistical errors for every measured quantity.

\subsection{Correlations}

In order to extract HQE parameters, a fit to the measured moments needs to take account of the correlations. With the three different kinds of uncertainties calculated, covariance matrices for each of them need to be computed. Starting from the

efficiency-corrected event yields, $N_{i}$, and their covariance matrix $V_{i j}(N)$, the covariance between two derived quantities $x$ and $y$ is

$$
V_{x y}=\sum_{i} \sum_{j} \frac{\partial x}{\partial N_{i}} \frac{\partial y}{\partial N_{j}} V_{i j}(N),
$$

where for the quantities of interest the derivatives are given in Equations [7.2 and [7.3. Here it is important to note that $\partial x / \partial N_{k}=0$ unless bin $k$ is within the energy interval used in defining the variable $x$. The corresponding correlations are given by $C_{x y} \equiv V_{x y} / \sqrt{V_{x x} V_{y y}}$.

The computation of the correlation matrix for the statistical errors is a straightforward application of the above equations, since the underlying matrix $V_{i j}$ is diagonal. The results are given in Table 7.8. It is essential to point out that the eight quantities are not independent. In particular, given the two moments for the highest energy cut 
and the number of events in each of the three lowest energy bins, one can compute all the other moments - so there are only five independent quantities.

\begin{tabular}{|cc|cccccccc|}
\hline Moment & & $\mathrm{M}$ & $\mathrm{M}$ & $\mathrm{M}$ & $\mathrm{M}$ & $\mathrm{V}$ & $\mathrm{V}$ & $\mathrm{V}$ & $\mathrm{V}$ \\
& Min. $E_{\gamma}$ & 1.9 & 2.0 & 2.1 & 2.2 & 1.9 & 2.0 & 2.1 & 2.2 \\
\hline $\mathrm{M}$ & 1.9 & 1.0000 & 0.5172 & 0.3548 & 0.2265 & -0.6110 & 0.0077 & 0.0821 & 0.1008 \\
$\mathrm{M}$ & 2.0 & & 1.0000 & 0.6838 & 0.4326 & 0.2285 & -0.0008 & 0.1375 & 0.1645 \\
$\mathrm{M}$ & 2.1 & & & 1.0000 & 0.6260 & 0.4220 & 0.5660 & 0.1650 & 0.1884 \\
$\mathrm{M}$ & 2.2 & & & & 1.0000 & 0.4528 & 0.7568 & 0.7383 & 0.2113 \\
$\mathrm{~V}$ & 1.9 & & & & & 1.0000 & 0.4626 & 0.3486 & 0.2520 \\
$\mathrm{~V}$ & 2.0 & & & & & & 1.0000 & 0.6966 & 0.4887 \\
$\mathrm{~V}$ & 2.1 & & & & & & & 1.0000 & 0.6709 \\
$\mathrm{~V}$ & 2.2 & & & & & & & & 1.0000 \\
\hline
\end{tabular}

Table 7.8: Correlation matrix for statistical errors on the truncated first (M) and second $(\mathrm{V})$ moments (four each, for different minimum $E_{\gamma}$ in $\mathrm{GeV}$ ).

The systematic uncertainties have three contributions. The first is from the uncertainty associated with the $B \bar{B}$ background subtraction. In order to compute this contribution to the total covariance matrix, the covariance matrix given in Table 4.23 is used in Equation 7.4. The second contribution is from the energy-scale uncertainty, which is important for the first moments (Table [7.4), but which has an insigificant effect for the second moments. Therefore, within the four-dimensional space of the first moments, a fully-correlated four-by-four matrix is added with element $k l$ equal to the product of the energy-scale errors for the $k^{\text {th }}$ and $l^{\text {th }}$ first moments, as given in Table 7.4. The remaining three quadrants of the eight-by-eight covariance matrix have no contribution from this effect. The third contribution is from the calorimeter resolution uncertainty, which is barely significant for the second moments, but negligible for the first moments. This contibution is treated in an analogous fashion to the energy-scale contribution, this time within the four-dimensional space of the second moments. Finally, after the full covariance matrix has been constructed by the addition of the three components, the correlation matrix is computed (Table [7.9).

The computation of the correlations among the model-dependence uncertainties is less straightforward. The first moments have two kinds of model-dependence uncertainties, one from the dependence of the selection efficiency (Section 17.1) and the 


\begin{tabular}{|rr|cccccccc|}
\hline Moment & & $\mathrm{M}$ & $\mathrm{M}$ & $\mathrm{M}$ & $\mathrm{M}$ & $\mathrm{V}$ & $\mathrm{V}$ & $\mathrm{V}$ & $\mathrm{V}$ \\
& Min. $E_{\gamma}$ & 1.9 & 2.0 & 2.1 & 2.2 & 1.9 & 2.0 & 2.1 & 2.2 \\
\hline $\mathrm{M}$ & 1.9 & 1.0000 & 0.7841 & 0.6076 & 0.4622 & -0.7552 & -0.3862 & -0.1723 & -0.0942 \\
$\mathrm{M}$ & 2.0 & & 1.0000 & 0.8007 & 0.6311 & -0.2515 & -0.4753 & -0.2298 & -0.1451 \\
$\mathrm{M}$ & 2.1 & & & 1.0000 & 0.7886 & -0.0320 & 0.0202 & -0.2903 & -0.2115 \\
$\mathrm{M}$ & 2.2 & & & & 1.0000 & 0.0489 & 0.1850 & 0.2061 & -0.1001 \\
$\mathrm{~V}$ & 1.9 & & & & & 1.0000 & 0.4473 & 0.2219 & 0.1436 \\
$\mathrm{~V}$ & 2.0 & & & & & & 1.0000 & 0.5003 & 0.3342 \\
$\mathrm{~V}$ & 2.1 & & & & & & & 1.0000 & 0.6592 \\
$\mathrm{~V}$ & 2.2 & & & & & & & & 1.0000 \\
\hline
\end{tabular}

Table 7.9: Correlation matrix for systematic errors on the truncated first (M) and second $(\mathrm{V})$ moments (four each, for different minimum $E_{\gamma}$ in $\mathrm{GeV}$ ).

other from the dependence of the $\Delta_{\Upsilon \rightarrow B}$ corrections (Section 7.3). On the other hand, the efficiency-related uncertainty is negligible for the second moments. The treatment of these two kinds of model-dependence effects are also different.

Therefore, in order to estimate a covariance matrix, the models are treated a statistical ensemble. First, for each of $N_{\text {models }}=16$ models (four different parameterizations of the $\mathrm{KN}$ model and twelve of the BBU model), indexed $\alpha$, a $\Delta_{\text {eff }}^{\alpha}$ is computed as the difference between the value of a given moment measured using the efficiencies predicted by the $\alpha^{\text {th }}$ model and the value of that moment measured using the efficiencies predicted by the nominal KN460 model. Then this quantity is added to $\Delta_{\Upsilon \rightarrow B}^{\alpha}$ to arrive at a total correction:

$$
\Delta_{\text {total }}^{\alpha} \equiv \Delta_{\Upsilon \rightarrow B}^{\alpha}+\Delta_{e f f}^{\alpha} .
$$

Then the mean value of the total correction can be computed, along with the covariance between moments $x$ and $y$ :

$$
\begin{gathered}
\left\langle\Delta_{\text {total }}\right\rangle=\frac{1}{N_{\text {models }}} \sum_{\alpha} \Delta_{\text {total }}^{\alpha}, \\
V_{x y}=\frac{1}{N_{\text {models }}} \sum_{\alpha}\left(\Delta_{\text {total }}^{\alpha}(x)-\left\langle\Delta_{\text {total }}(x)\right\rangle\right) \cdot\left(\Delta_{\text {total }}^{\alpha}(y)-\left\langle\Delta_{\text {total }}(y)\right\rangle\right) .
\end{gathered}
$$


The correlation matrix can then be computed, as given in Table [7.10. While this method yields smaller uncertainties than the earlier conservative approach, it is used only in estimating the correlation matrix.

\begin{tabular}{|rr|cccccccc|}
\hline Moment & & $\mathrm{M}$ & $\mathrm{M}$ & $\mathrm{M}$ & $\mathrm{M}$ & $\mathrm{V}$ & $\mathrm{V}$ & $\mathrm{V}$ & $\mathrm{V}$ \\
& Min. $E_{\gamma}$ & 1.9 & 2.0 & 2.1 & 2.2 & 1.9 & 2.0 & 2.1 & 2.2 \\
\hline $\mathrm{M}$ & 1.9 & 1.0000 & 0.9267 & 0.9486 & 0.8252 & -0.9057 & -0.9223 & -0.9234 & -0.7983 \\
$\mathrm{M}$ & 2.0 & & 1.0000 & 0.9576 & 0.8669 & -0.7452 & -0.8183 & -0.8087 & -0.8960 \\
$\mathrm{M}$ & 2.1 & & & 1.0000 & 0.9540 & -0.7390 & -0.7889 & -0.7843 & -0.7752 \\
$\mathrm{M}$ & 2.2 & & & & 1.0000 & -0.5378 & -0.5857 & -0.5788 & -0.6023 \\
$\mathrm{~V}$ & 1.9 & & & & & 1.0000 & 0.9650 & 0.9824 & 0.6983 \\
$\mathrm{~V}$ & 2.0 & & & & & & 1.0000 & 0.9810 & 0.8035 \\
$\mathrm{~V}$ & 2.1 & & & & & & & 1.0000 & 0.8057 \\
$\mathrm{~V}$ & 2.2 & & & & & & & & 1.0000 \\
\hline
\end{tabular}

Table 7.10: Correlation matrix for model-dependence errors on the truncated first (M) and second (V) moments (four each, for different minimum $E_{\gamma}$ in $\left.\mathrm{GeV}\right)$.

\subsection{Extraction of HQE Parameters}

Using the obtained correlation matrices, it is possible to extract the parameters of the heavy quark expansion (HQE) from fits to the moments. This has been performed in the kinetic scheme [103]. The fit minimizes the $\chi^{2}$ defined by:

$$
\chi^{2}=\left(\vec{M}_{e x p}-\vec{M}_{H Q E}\right)^{T} C_{t o t}^{-1}\left(\vec{M}_{e x p}-\vec{M}_{H Q E}\right)
$$

where $\vec{M}_{\text {exp }}$ represents the moment measurements included in the fit and $\vec{M}_{H Q E}$ stands for their kinetic-scheme HQE prediction. $C_{t o t}=C_{\exp }+C_{t h e o}$ is the sum of the experimental and theoretical covariance matrices. The construction of the $C_{\text {theo }}$ matrix include variations in the coupling constant $\alpha_{S}(0.22 \pm 0.04)$, the HQE parameters $\mu_{\pi}^{2}\left(0.4 \mathrm{GeV}^{2} \pm 20 \%\right), \mu_{G}^{2}\left(0.35 \mathrm{GeV}^{2} \pm 20 \%\right), \rho_{D}^{3}\left(0.2 \mathrm{GeV}^{3} \pm 30 \%\right)$ and $\rho_{L S}^{3}\left(-0.15 \mathrm{GeV}^{3} \pm 30 \%\right)$. Additional theory errors have also been assigned on the masses of the heavy quarks $( \pm 20 \mathrm{MeV})$ and the bias corrections $( \pm 30 \%)$ predicted in the BBU model [25]. Finally half of the difference observed by using one or the 
other of the two different ansätze for the heavy-quark distribution function is linearly added as an extra theoretical uncertainty.

The fit to the first moments measured for $E_{\gamma}>1.9 \mathrm{GeV}$ and $E_{\gamma}>2.0 \mathrm{GeV}$ and the second moment measured for $E_{\gamma}>1.9 \mathrm{GeV}$ yields:

$$
m_{b}=(4.45 \pm 0.16) \mathrm{GeV} / c^{2} \quad \mu_{\pi}^{2}=(0.65 \pm 0.29) \mathrm{GeV}^{2}
$$

If the theoretical errors are not taken into account, the central value of the fit stays consistent, but the uncertainties are smaller:

$$
m_{b}=\left(4.46_{-0.10}^{+0.12}\right) \mathrm{GeV} / c^{2} \quad \mu_{\pi}^{2}=(0.64 \pm 0.20) \mathrm{GeV}^{2}
$$

The error ellipses from fits to various combinations are shown in Figure 7.3. The results from the different combinations are consistent with each other.
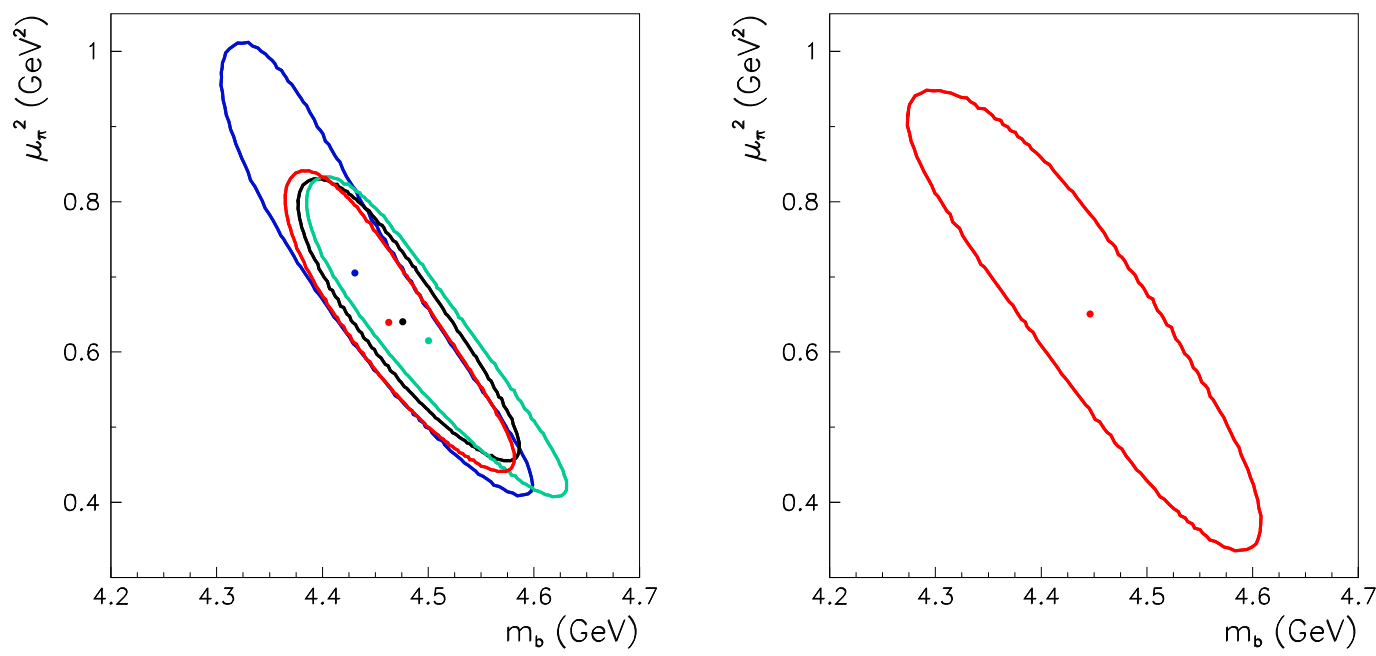

Figure 7.3: HQE parameters $m_{b}$ and $\mu_{\pi}^{2}$ from fits to the moments in the kinetic scheme [103]. Fit results are shown for different combinations of moments without the theory errors (left) and for the chosen combination with the theory errors (right). Blue, black and green ellipses show the fits to first and second moments with minimum photon energy of 1.9, 2.0 and $2.1 \mathrm{GeV}$, respectively. The red ellipse shows the fit to first moments for $E_{\gamma}>1.9 \mathrm{GeV}$ and $E_{\gamma}>2.0 \mathrm{GeV}$ and the second moment for $E_{\gamma}>1.9 \mathrm{GeV}$. 


\section{Chapter 8}

\section{Interpretation of Results}

\subsection{Photon Spectrum}

The photon spectrum for the inclusive decay $B \rightarrow X_{s} \gamma$, in the rest frame of the $\Upsilon(4 S)$ meson, is presented in Figure 7.2. This spectrum represents the sum of photons from $B \rightarrow X_{s} \gamma$ and $B \rightarrow X_{d} \gamma$ decays, but the contribution from the latter is expected to be only a few percent of the total. The measurement uncertainties in each energy bin are dominated by statistical errors, a result of the sizeable continuum background subtraction. The branching fraction, integrated in the interval from 1.9 to $2.7 \mathrm{GeV}$ is:

$\mathcal{B}(B \rightarrow X \gamma)^{1.9<E_{\gamma}^{*}<2.7 \mathrm{GeV}}=(3.64 \pm 0.29$ (stat) $\pm 0.33($ syst $) \pm 0.24($ model $)) \times 10^{-4}$

where the stated errors represent the statistical and the experimental systematic errors, and the uncertainty in the dependence of the detection efficiency on the assumed shape of the spectrum.

\subsection{Moments of the Photon Spectrum}

The first and second moments of the photon spectrum, fully-corrected for experimental resolution and translated from the rest frame of the $\Upsilon(4 S)$ resonance to that 
of the $B$-meson, are given in Table 8.1 as a function of the minimum photon energy. A comparison of these moments and earlier measurements with the prediction of non-perturbative QCD calculations is shown in Figure 8.1. It should be emphasized that the two measurements from BABAR (the sum-of-exclusive-modes and this fully-inclusive) are essentially independent from each other; there is almost no overlap in either statistical or systematic errors. The QCD calculations are based on heavy quark expansion (HQE) in the kinetic scheme by Benson, Bigi and Uraltsev (BBU) [25]. The predictions are computed using HQE parameters derived from $B A B A R$ measurements of moments of the hadronic mass and lepton energy spectra in $B \rightarrow X_{c} e \nu$ decays [107].

\begin{tabular}{|c|c|c|}
\hline $\begin{array}{c}E_{\min } \\
(\mathrm{GeV})\end{array}$ & $\begin{array}{c}\left\langle E_{\gamma}\right\rangle \pm \text { stat } \pm \text { sys } \pm \text { model } \\
(\mathrm{GeV}) \text { for } E_{\gamma}>E_{\min }\end{array}$ & $\begin{array}{c}\left\langle\left(E_{\gamma}-\left\langle E_{\gamma}\right\rangle\right)^{2}\right\rangle \pm \text { stat } \pm \text { sys } \pm \text { model } \\
\left(\mathrm{GeV}^{2}\right) \text { for } E_{\gamma}>E_{\min }\end{array}$ \\
\hline 1.9 & $2.288 \pm 0.025 \pm 0.017 \pm 0.012$ & $0.0328 \pm 0.0049 \pm 0.0023 \pm 0.0025$ \\
2.0 & $2.316 \pm 0.016 \pm 0.010 \pm 0.012$ & $0.0266 \pm 0.0026 \pm 0.0010 \pm 0.0020$ \\
2.1 & $2.355 \pm 0.014 \pm 0.007 \pm 0.010$ & $0.0191 \pm 0.0019 \pm 0.0006 \pm 0.0015$ \\
2.2 & $2.407 \pm 0.012 \pm 0.005 \pm 0.008$ & $0.0116 \pm 0.0014 \pm 0.0004 \pm 0.0005$ \\
\hline
\end{tabular}

Table 8.1: The measured first and centralized-second moments of the photon energy spectrum as a function of the minimum energy, $E_{m i n}$, required.

The measured moments are in good agreement with the earlier measurements and with theory predictions. The total uncertainties, particularly for the second moment measurement, are significantly smaller than CLEO and the BABAR sum-of-exclusive measurements. An extrapolation suggests that if the signal region were extended down to $1.8 \mathrm{GeV}$, the uncertainties would be comparable to those of the Belle measurement, even though the present analysis is based on a dataset that is approximately 1.75 times smaller than the Belle sample, thus indicating the improvement in background suppression by the lepton tagging.

From a fit of the predictions of BBU to the measured moments, the HQE parameters (in the kinetic scheme) have been extracted:

$$
m_{b}=\left(4.46_{-0.10}^{+0.12}\right) \mathrm{GeV} / c^{2} \quad \text { and } \quad \mu_{\pi}^{2}=(0.64 \pm 0.20) \mathrm{GeV}^{2}
$$



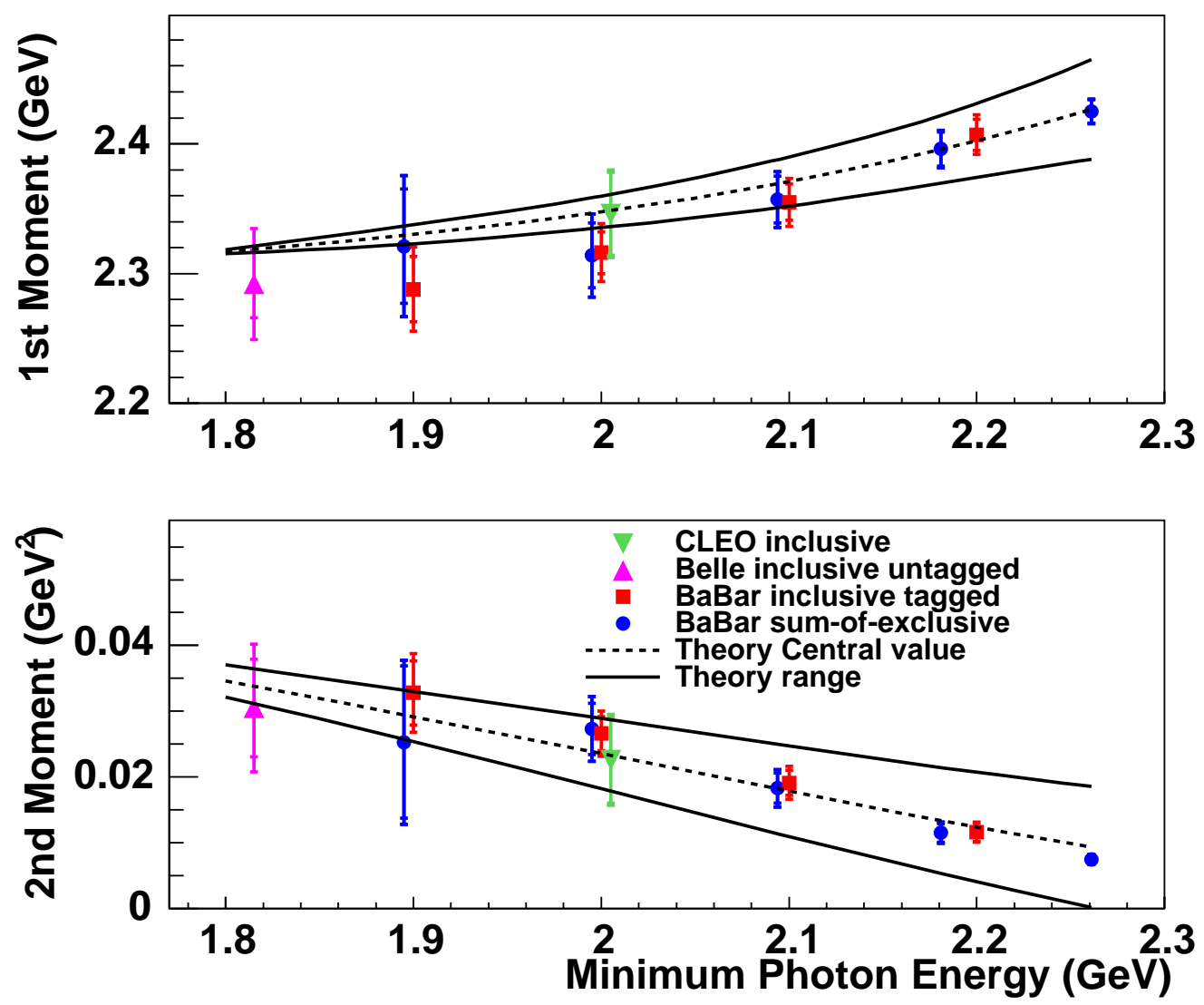

Figure 8.1: Comparison of the measured first and centralized-second moments with earlier results from the CLEO Collaboration [104], the Belle Collaboration [105] and the BABAR sum-of-exclusive-modes measurement [106]. The heavy quark expansion predictions are also plotted. 
These values are consistent with earlier measurements and the errors are comparable.

\subsection{Total Branching Fraction}

Using the extracted HQE parameters, the measured spectrum above $1.9 \mathrm{GeV}$ can be extrapolated to the lower limit of the spectrum. To account for the inclusive $B \rightarrow X_{d} \gamma$ contribution, the extrapolated value is reduced by $(4.0 \pm 1.6) \%$, since this $B \rightarrow X_{d} \gamma$ contribution would scale as $\left|V_{t d} / V_{t s}\right|^{2}$. After this adjustment the total $B \rightarrow X_{s} \gamma$ branching fraction is:

$$
\mathcal{B}\left(B \rightarrow X_{s} \gamma\right)^{E_{\gamma}>1.6 \mathrm{GeV}}=(4.05 \pm 0.32(\text { stat }) \pm 0.38(\text { syst }) \pm 0.29(\text { model })) \times 10^{-4}
$$

This total branching fraction is in agreement with the earlier measurements (Figure 8.2) and with the next-to-leading-order Standard Model calculation:

$$
\mathcal{B}\left(B \rightarrow X_{s} \gamma\right)^{E_{\gamma}>1.6 \mathrm{GeV}}=\left(\left.3.61_{-0.40}^{+0.24}\right|_{\frac{m_{c}}{m_{b}}} \pm 0.02_{\mathrm{CKM}} \pm 0.24_{\text {param. }} \pm 0.14_{\text {scale }}\right) \times 10^{-4}
$$

The new world average, including the results of this experiment, implies important constraints on various extensions of the Standard Model. While the constraints on the supersymmetric extensions are strongly dependent on the parameters of the particular models [17], the good agreement between the measurement and the Standard Model prediction implies less parameter-dependent constraints on simpler extensions. One such extension is the type-II two-Higgs-doublet model. The new world average, $\mathcal{B}\left(B \rightarrow X_{s} \gamma\right)^{E_{\gamma}>1.6 \mathrm{GeV}}=(3.55 \pm 0.26) \times 10^{-4}$, corresponds to a minimum mass of $620 \mathrm{GeV}$ (at $90 \%$ confidence level) for the charged Higgs boson predicted in this model [108].

\subsection{Future Prospects}

A significant advantage of the technique presented here is the scaling of the systematic errors, which are dominated by the subtraction of the background from $B$-meson decays, in almost the same way as the statistical errors scale with the number of 
BaBar

fully inclusive

BaBar

sum-of-exclusive

CLEO

mixed technique

BELLE

fully inclusive

ALEPH

sum-of-exclusive

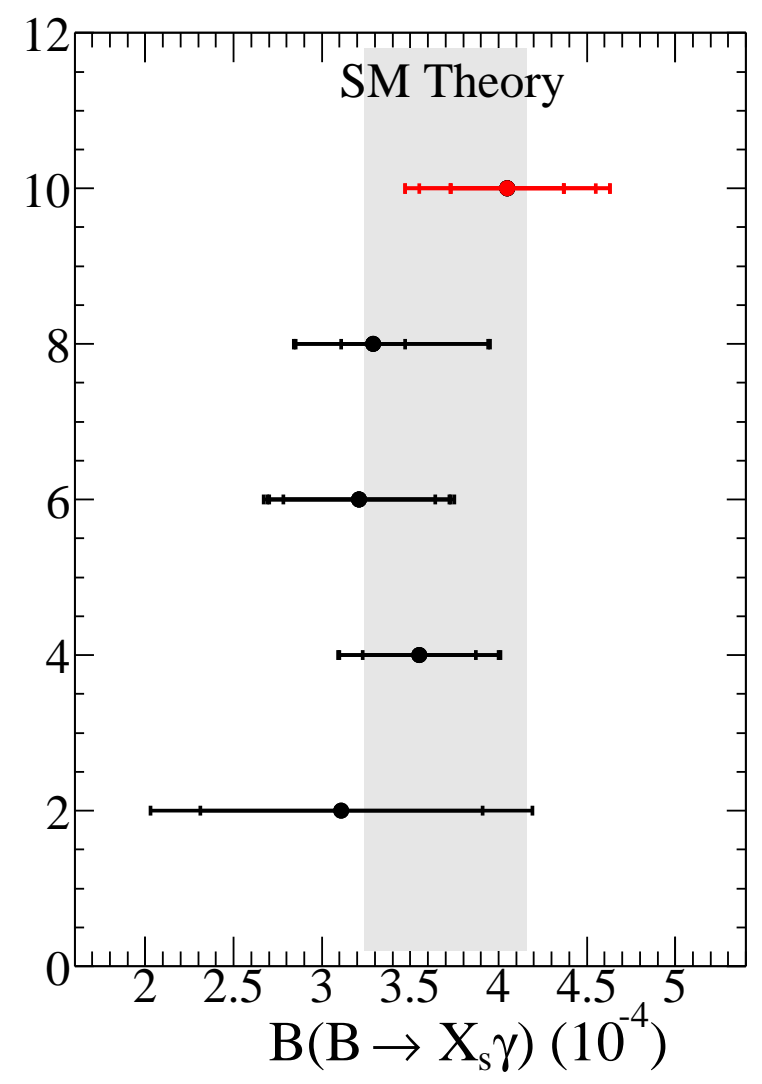

Figure 8.2: Comparison of the total branching fraction (for $E_{\gamma}>1.6 \mathrm{GeV}$ ) with the Standard Model prediction and with the earlier results from the ALEPH Collaboration [31], the CLEO Collaboration [104], the Belle Collaboration [105] and the BABAR sum-of-exclusive-modes measurement [106].

\begin{tabular}{|l|l|}
\hline Experiment & \multicolumn{1}{|c|}{$\mathcal{B}\left(B \rightarrow X_{s} \gamma\right) \times 10^{-4}$} \\
\hline This measurement & $4.05 \pm 0.32($ stat $) \pm 0.38($ syst $) \pm 0.29(t h)$ \\
BABAR sum-of-exclusive [106] & $3.29 \pm 0.18(\text { stat })_{-0.40}^{+0.62}(\text { syst })_{-0.23}^{+0.44}(t h)$ \\
CLEO mixed technique [104] & $3.21 \pm 0.43($ stat $) \pm 0.27(\text { syst })_{-0.10}^{+0.18}(t h)$ \\
BELLE fully inclusive [105] & $3.55 \pm 0.32($ stat $) \pm 0.31(\text { syst })_{-0.07}^{+0.11}($ th $)$ \\
ALEPH sum-of-exclusive [31] & $3.11 \pm 0.80($ stat $) \pm 0.72($ syst $)$ \\
\hline World Average & $3.55 \pm 0.26$ \\
\hline
\end{tabular}

Table 8.2: Comparison of the total branching fraction (for $E_{\gamma}>1.6 \mathrm{GeV}$ ) with other measurements. 
events. With the BABAR detector continuing to accumulate data at an increasing rate, the current data sample is expected to grow by an order of magnitude. Therefore both the statistical and systematic errors are likely to be improved by a factor of three or more.

The errors associated with the extrapolation calculations will similarly improve, as the fits to the HQE parameters reach higher precision. Furthermore, the dependence of the detection criteria on the assumed spectrum can be significantly decreased by defining a new optimization criterion that reduces the dependence of the selection efficiency on the photon energy.

With the precise measurement of the photon spectrum, much improved tests of the non-perturbative QED calculations will be possible and this will lead to significant improvements in the determination of $\left|V_{u b}\right|$.

The next-to-next-to-leading-logarithm calculation for the total branching is expected to be completed in the next two years. This is likely to reduce the theoretical uncertainty by a factor of two. With this in hand, the test of the Standard Model predictions will become much more sensitive. 


\section{Appendix A}

\section{Signal Model Parameters}

\section{A.1 The Kinetic Scheme}

The Benson-Bigi-Uraltsev calculation has five input parameters, which are set at the scale $\mu=1 \mathrm{GeV}$ that separates long and short-distance QCD effects, and hence are not directly comparable with the parameters of the Kagan and Neubert calculation. The first of these parameters, $m_{b}(\mu)$, is the running $b$-quark mass in this kinetic scheme. The next two, $\mu_{\pi}^{2}(\mu)$ and $\mu_{G}^{2}(\mu)$, which enter the heavy quark expansion for the spectrum moments at order $\mathcal{O}\left(1 / m_{b}^{2}\right)$, are the matrix elements for the squared $b$-quark momentum operator, and for the chromomagnetic operator, as discussed in Section [1.6] Finally, the two remaining parameters, $\rho_{D}^{3}(\mu)$ and $\rho_{L S}^{3}(\mu)$, are the expectation values of the Darwin and spin-orbit (also called convection current) dimension-six operators, which enter the expansion at third order in $1 / m_{b}$. These operators, shown as the third and forth operators in Equation 1.8 are the exact QCD counterparts of the more familiar operators of the same name from nonrelativistic quantum mechanics and atomic physics [20, with the Darwin operator arising due to the $b$-quark moving in a spatially varying background gluon field.

For systematic studies, the values of the first three parameters are varied about the central values extracted from the BABAR fit to the hadronic-mass and lepton-energy distributions in semileptonic $B$-meson decays [107], whereas the values of the two $\rho$ parameters are kept constant at their extracted values. Table A.1 summarizes the 


\begin{tabular}{|c|c|c|c|c|}
\hline Model & $m_{b}\left(\mathrm{GeV} / c^{2}\right)$ & $\mu_{\pi}^{2}\left(\mathrm{GeV}^{2}\right)$ & $\mu_{G}^{2}\left(\mathrm{GeV}^{2}\right)$ & $f_{K^{*}}$ \\
\hline BBU01 & 4.60 & 0.45 & 0.35 & 0.1115 \\
BBU02 & 4.60 & 0.45 & 0.27 & 0.1191 \\
BBU03 & 4.60 & 0.35 & 0.35 & 0.0604 \\
BBU04 & 4.60 & 0.55 & 0.35 & 0.1605 \\
BBU05 & 4.60 & 0.65 & 0.35 & 0.2051 \\
BBU06 & 4.50 & 0.45 & 0.35 & 0.0478 \\
BBU07 & 4.70 & 0.45 & 0.35 & 0.2150 \\
BBU08 & 4.45 & 0.55 & 0.27 & 0.0601 \\
BBU09 & 4.55 & 0.45 & 0.27 & 0.0803 \\
BBU10 & 4.65 & 0.35 & 0.27 & 0.1061 \\
BBU11 & 4.70 & 0.30 & 0.27 & 0.1205 \\
BBU12 & 4.75 & 0.25 & 0.27 & 0.1346 \\
\hline
\end{tabular}

Table A.1: The input parameters to the Benson-Bigi-Uraltsev $B \rightarrow X_{s} \gamma$ calculations [25], $m_{b}, \mu_{\pi}^{2}$ and $\mu_{G}^{2}$, used in the weighting of the flat Monte Carlo samples. The untabulated input parameters are kept constant: $\rho_{D}^{3}=0.2 \mathrm{GeV}^{3}$ and $\rho_{L S}^{3}=-0.09 \mathrm{GeV}^{3}$. Also tabulated is the $K^{*}(892) \gamma$ fraction predicted for a given set of input parameters, i.e., the integral of the distribution below $m_{\text {cutoff }}=1.1 \mathrm{GeV}$ normalized to the total.

various combinations of the values used for all the parameters. There are two types of variations considered. In the first type (BBU01-07), $m_{b}, \mu_{\pi}^{2}$ and $\mu_{G}^{2}$ are varied one-byone independently. For the combinations BBU08-12, $\mu_{\pi}^{2}$ is decreased with increasing $m_{b}$; such a correlation is considered to have a better physics motivation.

The variations in the parameters are conservatively large. Variations of $m_{b}$ and $\mu_{\pi}^{2}$ have been limited mainly to avoid $K^{*}$ fractions, $f_{K^{*}}$, more than about a factor of two from measured values (table A.1). The two $\mu_{G}^{2}$ values used are based on the BABAR fit [107] and $B$ - $B^{*}$ mass splitting [109. The dependence of the moments to this parameter is quite weak. A theoretical bound, $\mu_{\pi}^{2}>\mu_{G}^{2}$ [109], is observed to the extent possible within the tables provided by Benson [110].

These parameters fix the mean and the variance of the heavy quark distribution function in the BBU model. However, that still leaves some arbitrariness in the exact form of this function, which in turn determines the size of the bias corrections that need to be introduced to account for the experimental cut on the minimum photon 
energy. Reference [25] introduces two classes of heavy-quark distribution functions to estimate the theoretical uncertainty due to this arbitrariness. In the BBU weighting of the flat Monte Carlo samples for the determination of the experimental efficiencies, just one of the two, $F_{1}\left(k_{+}\right)$, is used, since the other yields very similar results for the values of $m_{b}$ and $\mu_{\pi}^{2}$ from Reference [107, but exhibits unphysical behaviour when $m_{b}$ and $\mu_{\pi}^{2}$ are high.

\section{A.2 The Shape Function Scheme}

The calculations of Lange, Neubert and Paz [26] rely upon QCD factorization to separate the problem into quantities which have clean meanings at different mass scales. The non-perturbative hadronic structure is embodied in the shape function; in this shape function scheme, it is renormalized at an intermediate scale $\mu_{i} \sim \sqrt{m_{B} \Lambda_{Q C D}}$. The computation is done to next-to-leading-order precision. The HQET parameters $m_{b} \equiv m_{B}-\bar{\Lambda}$ and $\mu_{\pi}^{2}$ are easily defined in terms of truncated moments of the shape function, and hence in this form depend on both $\mu_{i}$ and the factorization scale, the latter corresponding to the maximum photon energy $\left(E_{\gamma}\right)$ range accessible to experiments. But the authors convert these values to parameters depending only on a single scale, which they choose to be $\mu=1.5 \mathrm{GeV}$.

Computations in this theory require a parameterized input form for the shape function; this will allow predictions to be fit to the data to extract HQET parameters. The authors provide three parameterizations, called "exponential" (the default), "Gaussian" and "hyperbolic". Each involves two parameters, $b$ and $\Lambda$, but these parameters have no fundamental significance, nor are those for one functional form related to those for another form. The important quantities are the single-scale $m_{b}$ and $\mu_{\pi}^{2}$, which can be computed for each specific shape function.

For systematic studies, eleven particular parameterizations for the exponential shape function are selected to weight of the flat Monte Carlo sample. These are listed in table A.2. which shows the input parameters, the consequent HQET parameters (at $\mu=1.5 \mathrm{GeV}$ ), and the $K^{*}(892) \gamma$ fraction for each model. The latter fractions have been computed in exactly the same way as for the KN and BBU-based models, 
and the range of parameter space covered has again been limited by a requirement to keep the $K^{*} \gamma$ fractions reasonable.

\begin{tabular}{|c|c|c|c|c|c|}
\hline Model & $b$ & $\Lambda(\mathrm{GeV})$ & $m_{b}(\mathrm{GeV})$ & $\mu_{\pi}^{2}\left(\mathrm{GeV}^{2}\right)$ & $f_{K^{*}}$ \\
\hline LNP01 & 4.000 & 0.900 & 4.472 & 0.290 & 0.0386 \\
LNP02 & 2.750 & 0.900 & 4.520 & 0.365 & 0.0906 \\
LNP03 & 2.750 & 1.000 & 4.468 & 0.396 & 0.0753 \\
LNP04 & 5.000 & 0.700 & 4.624 & 0.129 & 0.0470 \\
LNP05 & 4.000 & 0.750 & 4.588 & 0.213 & 0.0634 \\
LNP06 & 3.000 & 0.800 & 4.572 & 0.304 & 0.0961 \\
LNP07 & 2.000 & 0.850 & 4.595 & 0.405 & 0.1649 \\
LNP08 & 5.000 & 0.550 & 4.766 & 0.038 & 0.1021 \\
LNP09 & 3.750 & 0.550 & 4.767 & 0.096 & 0.1462 \\
LNP10 & 2.500 & 0.550 & 4.775 & 0.188 & 0.2380 \\
LNP11 & 2.000 & 0.650 & 4.711 & 0.307 & 0.2310 \\
\hline
\end{tabular}

Table A.2: The parameters, $b$ and $\Lambda$, of the "exponential" shape functions in the Lange-Neubert-Paz $B \rightarrow X_{s} \gamma$ calculations [26] used to weight of the flat Monte Carlo samples. Also shown are the implied values of $m_{b}$ and $\mu_{\pi}^{2}$, which do not have the same meanings in this shape function scheme as in the kinetic scheme of BBU [25]. Also tabulated is the $K^{*}(892) \gamma$ fraction predicted for a given set of input parameters, i.e., the integral of the distribution below $m_{\text {cutoff }}=1.1 \mathrm{GeV}$ normalized to the total (the integral with $E_{\gamma}>1.6 \mathrm{GeV}$ ). 


\section{Appendix B}

\section{VCS Samples}

Virtual Compton Scattering (VCS) samples are used as a clean source of photons for various systematic studies. These events have the same final state, $e^{+} e^{-} \gamma$, as radiative Bhabha processes, but, instead of the photon being emitted from one of the external lepton lines, it is characterized by the elastic scattering of one electron from a quasi-real photon emitted by the other electron. The latter electron continues close to the beam line, with a momentum distribution strongly and narrowly peaked along the beam line; while for large scattering angles the first electron and the photon can appear at opposite azimuths in the detector. Radiative corrections do not significantly change the peaking behavior. Because of the simple kinematics, the energies of the detected particles can be predicted from their angles. Hence VCS events provide a clean sample of known-energy photons in an energy range appropriate for the $B \rightarrow X_{s} \gamma$ analysis. These photons can be used to compare data and simulation for resolution, shower shape and other properties of the detected photons.

The VCS event selection requires exactly one charged and one neutral particle candidate exist in each event, since the electron emitting the photon falls outside of the detector acceptance. Uncalibrated energy of the photon candidate is required to be at least $1.4 \mathrm{GeV}$ in the laboratory frame. A veto on polar acollinearity rejects contamination from Bhabha events with hard Bremsstrahlung. Some additional kinematic requirements are used to clean the sample further [111]. Finally, the calibrated energy of the photons in the c.m. frame is required to be in the range $2.0<E_{\gamma}^{*}<2.7 \mathrm{GeV}$. 

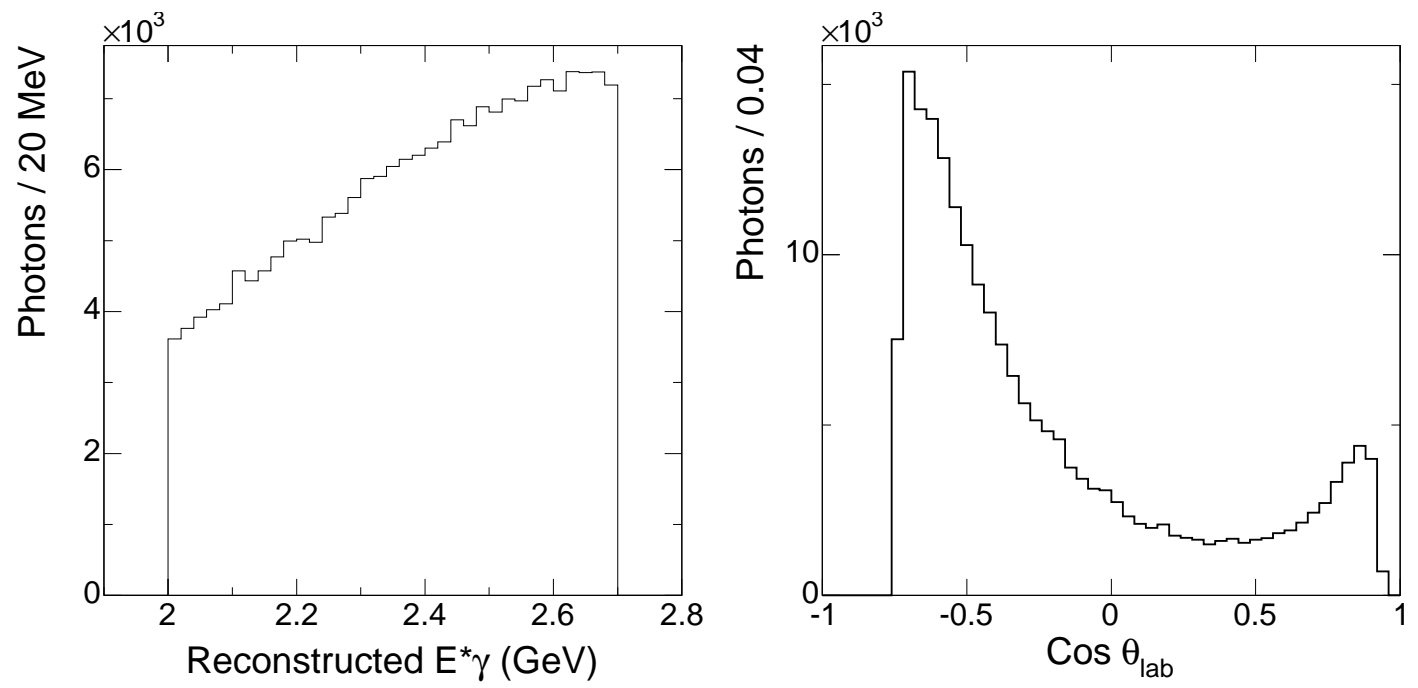

Figure B.1: Distributions for the c.m.-frame energy (left) and laboratoryframe polar angle (right) for photons from the VCS data sample.

About 200 thousand VCS photons, chosen randomly from a subset of the data collected in September 2001, are compared to a small sample of approximately 2400 simulated photons from Monte Carlo VCS events created using a generator initially developed for the SND experiment [112]. For the detector simulation the regular BABAR GEANT4 framework is used. Since the distribution of VCS photons in lab polar angle and $E_{\gamma}^{*}$ are different from those expected for high-energy photons in the $B \rightarrow X_{s} \gamma$ signal B.1, the events are reweighted first in lab polar angle, then in $E_{\gamma}^{*}$ to match the respective distributions from the $B \rightarrow X_{s} \gamma$ signal samples. 


\section{Bibliography}

[1] A discussion of the arbitrariness of the free parameters of the Standard Model is given in R. N. Cahn, Rev. Mod. Phys. 68, 951 (1996).

[2] B. A. Campbell and P. J. O'Donnell, Phys. Rev. D 25, 1989 (1982).

[3] J. L. Hewett, Top Ten Models Constrained by $b \rightarrow s \gamma$, Proceedings of the $21^{\text {st }}$ SLAC Summer Institute on Particle Physics: Spin Structure in High Energy Processes, SLAC-R-444 463 (1993).

[4] S. Eidelman et al., Phys. Lett. B 592, 1 (2004).

[5] M. Shifman, Quark-Hadron Duality, hep-ph/0009131.

[6] A. J. Buras, Weak Hamiltonian, CP Violation and Rare Decays, hep-ph/9806471

[7] T. Hurth, Rev. Mod. Phys. 75, 1159 (2003).

[8] K. Adel and Y. P. Yao, Phys. Rev. D 49, 4945 (1994) C. Greub and T. Hurth, Phys. Rev. D 56, 2934 (1997); A. J. Buras et al., Nucl. Phys. B 517, 353 (1998); M. Ciuchini et al., Nucl. Phys. B 527, 21 (1998); C. Bobeth et al., Nucl. Phys. B 574, 291 (2000).

[9] G. Altarelli et al., Nucl. Phys. B 187, 461 (1981); A. J. Buras and P. H. Weisz, Nucl. Phys. B 333, 66 (1990); A. J. Buras et al., Nucl. Phys. B 370, 69 (1992); Nucl. Phys. B 375, 501 (1992); Nucl. Phys. B 400, 37 (1993); M. Ciuchini et al., Phys. Lett. B 301, 263 (1993); Nucl. Phys. B 415, 403 (1994); M. Misiak and M. Münz, Phys. Lett. B 344, 308 (1995); 
K. G. Chetyrkin et al., Phys. Lett. B 400, 206 (1997); Phys. Lett. B 425, 414(E) (1998); Nucl. Phys. B 518, 473 (1998); Nucl. Phys. B 520, 279 (1998); P. Gambino et al., Nucl. Phys. B 673, 238 (2003).

[10] C. Greub et al., Phys. Lett. B 380, 385 (1996); Phys. Rev. D 54, 3350 (1996); A. J. Buras et al., Nucl. Phys. B 611, 488 (2001).

[11] A. Czarnecki and W. J. Marciano, Phys. Lett. B 81, 277 (1998); K. Baranowski and M. Misiak, Phys. Lett. B 481, 410 (2000); P. Gambino and U. Haisch, JHEP 0009, 001 (2000); JHEP 0110, 020 (2001); A. Strumia, Nucl. Phys. B 532, 28 (1998).

[12] The branching fraction is mostly quoted with a minimum energy of $1.6 \mathrm{GeV}$ in the $B$-meson rest frame. With this cutoff, the mass of the final $X_{s}$ state is constrained to be smaller than $m_{\eta_{c}}+m_{K}$ and hence real $c \bar{c}$ intermediate states might occur only in cascade decays $\left(B \rightarrow Y_{c \bar{c}} X_{s}\right.$ followed by $\left.Y_{c \bar{c}} \rightarrow X \gamma\right)$, which are treated as backgrounds. With this choice, non-perturbative corrections to the theoretical decay rate are considered to be under better control.

A. J. Buras and M. Misiak, Acta. Phys. Polon. B33, 2597 (2002), T. Hurth et al., Nucl. Phys. B 704, 56 (2005).

[13] H. M. Asatrian et al., Phys. Lett. B 619, 322 (2005).

[14] M. Drees, An Introduction to Supersymmetry, hep-ph/9611409; H. Murayama, Supersymmetry Phenomenology, hep-ph/0002232.

[15] K. Lane, Technicolor 2000, hep-ph/0007304 C. T. Hill and E. H. Simmons, Phys. Rep. 381, 235 (2003).

[16] ATLAS Collaboration, Detector and Physics Performance Technical Design Report, Volume II, CERN/LHCC 99-15 (1999); CMS Collaboration, S. Abdullin et al., J. Phys. G 28469 (2002).

[17] H. Baer et al., JHEP 0306, 054 (2003). 
[18] M. Ciuchini et al., Nucl. Phys. B 534, 3 (1998); C. Bobeth et al., Nucl. Phys. B 567, 153 (2000); G. Degrassi et al., JHEP 0012, 009 (2000); M. Carena et al., Phys. Lett. B 499, 141 (2001); D. A. Demir and K. A. Olive, Phys. Rev. D 65, 034007 (2002).

[19] M. Shifman, Lectures on Heavy Quarks in Quantum Chromodynamics, extended version of the lectures given at Theoretical Advanced Study Institute QCD and Beyond, University of Colorado, Boulder, Colorado, hep-ph/9510377; I. Bigi et al., Ann. Rev. Nucl. Part. Sci. 47591 (1997); N. Uraltsev, Topics in the Heavy Quark Expansion, published in M. Shifman (ed.), At the Frontier of Particle Physics - Handbook of QCD - Boris Ioffe Festschrift, World Scientific, Singapore (2001).

[20] J.D. Bjorken and S.D. Drell, Relativistic Quantum Mechanics, McGraw-Hill, New York (1964).

[21] A. Ali and C. Greub, Phys. Lett. B 361, 146 (1995).

[22] M. Neubert, Phys. Rev. D 49, 4623 (1994) Phys. Rev. D 49, 3392 (1994).

[23] A. Kagan and M. Neubert, Euro. Phys. J. 7, 5 (1999).

[24] The Kagan and Neubert calculation actually conforms to the heavy quark effective theory (HQET), an alternative approach to heavy quark expansion. The HQET uses the fields $h(x)=\frac{1+\gamma_{0}}{2} \tilde{Q}(x)$, instead of the $\varphi_{Q}(x)$ fields defined in Equation [1.9. Up to order $1 / m_{Q}$, the interpretation of the results from HQET are the same as HQE. A description of the differences is given in Reference [19]. A review of HQET is given in: A. F. Falk, The Heavy Quark Expansion of QCD, Proceedings of the $24^{\text {th }}$ SLAC Summer Institute on Particle Physics: The Strong Interaction, From Hadrons to Partons, SLAC-R-508 43 (1996).

[25] D. Benson, I. Bigi and N. Uraltsev, Nucl. Phys. B 710, 371 (2005).

[26] B. O. Lange, M. Neubert and G. Paz, Phys. Rev. D 72, 073006 (2005), This work builds on the paper of S. W. Bosch et al., Nucl. Phys. B 699, 335 (2004). 
[27] Veseli and Olsson predict that various $K^{*}$ resonances account for $(37 \pm 14) \%$ of the total $B \rightarrow X_{s} \gamma$ decay rate. Apart from the $K^{*}(892) \gamma$, so far five resonant final states have been observed: $K_{2}^{*}(1430) \gamma, K_{1}(1270) \gamma, K \phi \gamma, K \eta \gamma, \Lambda \bar{p} \gamma$.

S. Veseli and M.G. Olsson, Phys. Lett. B 367, 309 (1996); Belle Collaboration, S. Nishida et al., Phys. Rev. Lett. 89, 231801 (2002); BABAR Collaboration, B. Aubert et al., Phys. Rev. D 70, 091105 (2004); Belle Collaboration, A. Drutskoy et al., Phys. Rev. Lett. 92, 051801 (2004); Belle Collaboration, H. Yang et al., Phys. Rev. Lett. 94, 111802 (2005); Belle Collaboration, S. Nishida et al., Phys. Lett. B 610, 23 (2005); Belle Collaboration, K. Abe et al., Observation of $B^{+} \rightarrow p \bar{\Lambda} \gamma$, contributed to the $32^{\text {nd }}$ International Conference on High-Energy Physics, 2004, Beijing, China, hep-ex/0409009.

[28] BABAR Collaboration, B. Aubert et al., $B \rightarrow X_{s} \gamma$ Using a Sum of Exclusive Modes, contributed to the $31^{\text {st }}$ International Conference on High-Energy Physics, Amsterdam, The Netherlands, SLAC-PUB-9308 (2002).

[29] BABAR Collaboration, B. Aubert et al., Phys. Rev. D 70, 112006 (2004).

[30] CLEO Collaboration, R. Ammar et al., Phys. Rev. Lett. 71, 674 (1993).

[31] ALEPH Collaboration, R. Barate et al., Phys. Lett. B 429, 169 (1998).

[32] S. Stone, $B$ Phenomenology, presented at the $55^{\text {th }}$ Scottish Universities Summer School in Physics on Heavy Flavor Physics, Scotland, 2001, hep-ph/0112008.

[33] BABAR Collaboration, B. Aubert et al., Nucl. Instrum. Methods A.479, 1 (2002).

[34] For $\Upsilon(4 S), \Gamma_{n o n-B \bar{B}} / \Gamma_{\text {Total }}<0.04$ at $95 \%$ confidence level. CLEO Collaboration, B. Barish et al., Phys. Rev. Lett. 76, 1570 (1996).

[35] W. Kozanecki et al., AIP Conf. Proc. 693, 235 (2003); A. Kulikov et al., SLAC-PUB-10886 (2004).

[36] PEP-II records, as of July 31, 2004, http://www.slac.stanford.edu/grp/ad/ pep-ii/stats/PEPII_lum_record.pdf 
[37] M. Bruinsma et al., Status and Prospects of the BABAR SVT, presented at Vertex 2004 Workshop, to appear in Nucl. Instrum. Methods A.

[38] K. Hagiwara et al., Phys. Rev. D 66, 010001 (2002),

While the exact value of the Molière radius for CsI crystals is inconsequential for the calibrations of the EMC, it is interesting to note that the more recent version of the Review of Particle Physics [4] lists a lower value, $3.5 \mathrm{~cm}$. The difference is due to the recomputation of the value of the critical energy used to relate the radiation length to the Molière radius. Actually this recomputation has not been performed consistently and the value will be corrected to $3.57 \mathrm{~cm}$ in the next edition of the Review.

C. Woody, private communication (2005).

[39] Cylinderical RPC layers differ in the details of construction and the choice of materials, but are based on the same concept. For more details refer to [33].

[40] J. Va'vra, BaBar RPCs and related topics, presented at NuMI NOvA Experiment Detector Workshop, 2003, Stanford, CA, http://www-nova.fnal.gov/ workshops/stanford03/transparencies/vavra_RPC_summary_2003_talk.pdf

[41] S. Jadach et al., hep-ph/9608412; Comput. Phys. Commun. 70, 305 (1992); Phys. Rev. D 40, 3582 (1989).

[42] S. Jadach and Z. Wạs, Comput. Phys. Commun. 85, 453 (1995).

[43] A. Ryd and D. Lange, Evtgen Documentation, BABAR Internal Analysis Document 522 (2003).

[44] T. Sjöstrand, Comput. Phys. Commun. 82, 74 (1994).

[45] E. Barberio and Z. Wạs, Comput. Phys. Commun. 79, 291 (1994).

[46] Geant4 Collaboration, S. Agostinelli et al., Nucl. Instrum. Methods A.506, 250 (2003). 
[47] The BABAR GHIT truth-matching technique is used, as detailed in:

R. Dubitzky, Recipe for using GHit based matching in micro analyses, 2002, http://www.slac.stanford.edu/ dubitzky/GHit_matcher.html;

The mismatch fraction for this technique is believed to be very low, since even for its predecessor, the mismatch ratio was about $1 \%$ for the soft pions in $D^{*} \rightarrow$ $D^{0} \pi_{\text {soft }}$ :

F. Simonetto, A study of the MC truth associator for soft pions from $D^{*}$ decays, BABAR Internal Analysis Document 523 (2002).

[48] The cross-sections for the Monte Carlo samples listed in Table 2.1 have 2-3\% uncertainty. However this uncertainty is not important for the measurement, since the continuum backgrounds are subtracted using the off-resonance data and the generic $B \bar{B}$ backgrounds are scaled using the total number of $B \bar{B}$ events instead of the integrated luminosity.

O. Igonkina, private communication (2005); N. J. P. Berger, private communication (2005).

[49] R. Gamet et al., Luminosity Measurement for the Run 1 and Run 2 data sample using Release 10 and SP4 Simulation, BABAR Internal Analysis Document 535 (2003).

[50] C. Hearty, Measurement of the Number of $\Upsilon(4 S)$ Mesons Produced in Run 1 (B Counting), BABAR Internal Analysis Document 134 (2001).

[51] In the measurement of $n_{B \bar{B}}$, it is assumed that $\Upsilon(4 S)$ mesons decay exclusively to $B \bar{B}$ pairs. A small hypothetical $q \bar{q}$ contribution of $1 \%$, i.e. $\mathcal{B}(\Upsilon(4 S) \rightarrow q \bar{q})=$ 0.01 , would imply that $n_{B \bar{B}}$ is overestimated by $0.74 \%$.

C. Hearty, Impact of $\Upsilon(4 S) \rightarrow q \bar{q}$ on $B$ counting, 2004, http://babarhn.slac.stanford.edu:5090/HyperNews/get/luminosity/106.html

[52] M. Bona et al., Report of the Tracking Efficiency Task Force, BABAR Internal Analysis Document 324 (2002). 
[53] T. Brandt, Likelihood Based Electron Identification, BABAR Internal Analysis Document 391 (2002).

[54] The requirement on the minimum number of crystals is mainly for removing certain signals that were caused by a bus glitch in the EMC read-out modules during the first year of data taking.

J. Coleman, Bizarre EMC events, 2003, http://babar-hn.slac.stanford.edu:5090/ $\mathrm{hn} / \mathrm{aux} / \mathrm{coleman} / \mathrm{pi0s} / \mathrm{ph} /$ eventDisplay/

[55] ARGUS Collaboration refers to the lateral moment as the fractional width, which is perhaps more appropriate, since it is a dimensionless measure of the shower shape.

A. Drescher et al., ARGUS Collaboration, Nucl. Instrum. Methods A.237, 464 (1985).

[56] BABAR Collaboration, B. Aubert et al., Determination of the Branching Fraction for Inclusive Decays $B \rightarrow X_{s} \gamma$, contributed to the $31^{\text {st }}$ International Conference on High-Energy Physics, Amsterdam, The Netherlands, SLAC-PUB-9301(2002).

[57] G. Fox and S. Wolfram, Phys. Rev. Lett. 41, 1581 (1978).

[58] R. A. Fisher, Ann. Eugenics 7, 179 (1936).

[59] Cornelius++, the $\mathrm{C}++$ implementation of Cornelius, is used for the computation of the Fisher discriminant.

A. Gaidot et al., Cornelius: Multi-Purpose Fitting Scheme, BABAR Internal Tagging Note 10 (1997).

[60] It has been reported that regardless of how tight the momentum criterion is, the efficiency of requiring a lepton in signal events cannot exceed the squareroot of the same quantity in continuum background events. Although this statement is probably correct, the additional requirements on the tag provide an extra order of magnitude suppression of the background, proving the method clearly advantageous. 
A. Limosani, $B \rightarrow X_{s} \gamma$ photon energy spectrum from Belle, presented at CKM 2005 Workshop on the Unitarity Triangle, San Diego, CA, http://ckm2005. ucsd.edu/WG/WG2/wed3/limosani-WG2_3.pdf

[61] Electron Identification Analysis Working Group, U. Langenegger (ed.), CutBased Electron Identification, BABAR Internal Analysis Document 90 (2001).

[62] R. Sinkus and T. Voss, Nucl. Instrum. Methods A.391, 360 (1997).

[63] Muon Identification Analysis Working Group, F. Fabozzi (ed.) and L. Lista (ed.), Muon Identification in the BABAR Experiment, BABAR Internal Analysis Document 60 (2000).

[64] Muon candidates that satisfy the VeryLoose requirements of the BABAR PidKaonSMSSelector are vetoed. This selector identifies a track as a kaon if and only if $l_{K} \geq l_{p}$ and $l_{k}>r_{\pi} l_{\pi}$, where $l_{K, \pi, p}$ is the likelihood of the track for being a kaon, pion or proton, and $r_{\pi}$ is a likelihood ratio that depends on the track's momentum: $r_{\pi}= \begin{cases}1 & \text { if } p \leq 3 \mathrm{GeV} / c . \\ 2 & \text { if } p>3 \mathrm{GeV} / c .\end{cases}$

The kaon identification efficiency of this selector about $80 \%$ in the momentum range $0.6<p<4.5 \mathrm{GeV} / c$, with $5-10 \%$ misidentification rate for pions. Details on the computation of the likelihood and the performance can be found in: G. Mancinelli, S. Spanier, Kaon Selection at the BABAR Experiment, BABAR Internal Analysis Document 116 (2001);

S. Spanier, Tuning of the PidKaonSMSSelector for 2001, http://babarhn.slac.stanford.edu:5090/HyperNews/RETIRED/get/partID/329.html

[65] BABAR Collaboration, B. Aubert et al., Phys. Rev. Lett. 94, 221803 (2005).

[66] The decision to use independent flat MC samples for the determination of the efficiency figures was validated, when a small defect in the generator for KN480 events was later discovered. The defect has an about $0.6 \%$ effect on the signal efficiencies.

J. Libby, Bug in KN generator, 2004, http://babar-hn.slac.stanford.edu:5090/ HyperNews/get/RadPenguinAnal/556.html 
[67] The linear scaling of the c.m. momentum of a hadron that carries an appreciable fraction of the total energy in the event is motivated by a comparison of the differential hadron production cross section in the BABAR off-resonance data to measurements from three other experiments running at different c.m. energies [76]. While there are some substantial deviations from scaling, a more sophisticated correction is not needed for the high-energy photon candidates from hadron decays in this analysis, since the effect is already at the level of few $\mathrm{MeV}$. Moreover, the linear scaling is also copacetic for ISR photons. The differential cross section of ISR events, $d \sigma / d x$, where $x \equiv 2 E_{\gamma}^{*} / \sqrt{s}$, has an approximately $\alpha / s$ dependence on the cm energy, $\sqrt{s}$, and thus is not significantly different in on-resonance and off-resonance data.

O. Buchmüller et al., Initial State Radiation: event selection, 1C fit, ISR luminosity, BABAR Internal Analysis Document 558 (2003).

[68] The Crystal Ball function, commonly used to model processes with radiative energy losses, is defined as:

$$
f(x ; C, \mu, \sigma, \alpha, n)=C \cdot \begin{cases}A \times\left(n / \alpha-\alpha+\frac{\mu-x}{\sigma}\right)^{-n} & \text { for } x \leq \mu-\sigma \alpha \\ \exp \left(-\frac{(x-\mu)^{2}}{2 \sigma^{2}}\right) & \text { for } x>\mu-\sigma \alpha\end{cases}
$$

where $A \equiv(n / \alpha)^{n} \exp \left(-\alpha^{2} / 2\right)$. $C$ is a normalization factor, $\mu$ and $\sigma$ are the peak position and width of the Gaussian portion of the function, the join parameter $\alpha$ specifies the number of $\sigma$ s below $\mu$ at which the function changes to the power function and $n$ is the exponent of the power function. The function and its first derivative are continuous at the transition.

J.E. Gaiser, Charmonium Spectroscopy from Radiative Decays of the $J / \psi$ and $\psi$, Ph.D. Thesis, SLAC-R-255 (1982).

[69] A. Gaidot et al., Measurement of Inclusive $\eta$ and $\omega$ Momentum Spectra and Multiplicities at BABAR", BABAR Internal Analysis Document 163 (2001).

[70] A. Hicheur and D. Boutigny, Study of the inclusive and semi-exclusive $\eta^{\prime}$ spectrum, BABAR Internal Analysis Document 179 (2001). 
Various problems later identified with the inclusive measurement in this analysis were accounted for after private communication with A. Hicheur (2003).

[71] J. Back, Track-cluster matching for the electromagnetic calorimeter using Detector Model, BABAR Internal Note 440 (1998).

J. Back, Track-Bump Matching Study for Real Data, 2000, http://www.slac.stanford.edu/BFROOT/www/Detector/Calorimeter/software/ trkmatch/data/trkmatch.html

[72] A.M. Eisner, Level 3 Bhabha Filters (2000), http://www.slac.stanford.edu/ BFROOT/www/Detector/Trigger/software/L3TFilters/filters_bhabha.ps

[73] T. Brandt, Measurement of the Inclusive Electron Spectrum, Moments and Branching Fraction for Semileptonic $B$ Meson Decays, BABAR Internal Analysis Document 636 (2003).

[74] G. Cavoto and G. Raven, Measurement of the absolute tracking efficiency for high momentum tracks, BABAR Internal Analysis Document 61 (2000).

[75] H. Fesefeldt, Simulation of Hadronic Showers, Physics and Application, RWTH Aachen report PITHA 85/2 (1985).

[76] T. Hadig et al., Production of Charged $\pi^{ \pm}, K^{ \pm}$and $p / \bar{p}$ in $e^{+} e^{-}$Annihilations at $\sqrt{s}=10.54 \mathrm{GeV}$ and in Hadronic $\Upsilon(4 S)$ Decays, BABAR Internal Analysis Document 610 (2003).

[77] D. Bailey, Checking Basic Characteristics of Hadronic Shower Parameterizations in GHEISHA / GEANT4, presented at ATLAS GEANT4 Workshop in 2000, http://www.physics.utoronto.ca/ dbailey/ATLAS_GEANT4_4Dec2000/

[78] The selection of antiprotons that are used in the calorimeter response studies rely mainly on kinematic constraints on the $\bar{\Lambda}$ decay. A loose PID likelihood requirement based on the detector response from all five subdetectors is also applied, but the calorimeter information used in the selection is unlikely to have a significant impact on the measurements. The purity of the sample is higher 
than $95 \%$.

M. Sokoloff, private communication (2003); BABAR Charged Particle Identification Analysis Working Group, Files and ntuples from BetaPidCalib, 2001, http://www.slac.stanford.edu/BFROOT/www/Physics/Tools/Pid/Ntuples/ ntuplesV2.html

[79] CLEO Collaboration, S. Anderson et al., Phys. Rev. Lett. 86, 2732 (2001).

[80] Z. Ligeti et al., Phys. Rev. D 60, 034019 (1999),

[81] K. Chetyrkin et al., Phys. Lett. B 400, 206 (1997); M. Misiak and M. Munz, Phys. Lett. B 344, 308 (1995).

[82] G. Altarelli et al., Nucl. Phys. B 208, 365 (1982).

[83] Z. Ligeti, private communication (2003).

[84] V. Lüth, private communication (2005); E. Richter-Was, Phys. Lett. B 303, 163 (1993).

[85] CLEO Collaboration, R. Balest et al., Phys. Rev. D 52, 2661 (1995) BABAR Collaboration, B. Aubert et al., Phys. Rev. D 67, 032002 (2003).

[86] C. Hearty, private communication (2005).

[87] BABAR Radiative Penguin Analysis Working Group, D. Best et al., High Energy Photons at BABAR, BABAR Internal Analysis Document 201 (2001).

[88] F. Bellini et al., Comparison between Monte Carlo and Data of the $\pi^{0}$ detection and reconstruction efficiency using $\tau$ 1-on-1 decays, BABAR Internal Analysis Document 378 (2003).

[89] F. Di Lodovico et al., Neutral (pi0s, gamma) Efficiencies, 2003, http://www.slac.stanford.edu/BFROOT/www/Physics/Analysis/AWG/ Neutrals/validation/results/results.html 
[90] C. L. Brown, An Inclusive Measurement of the Branching Fraction for $B \rightarrow X_{s} \gamma$ Using the Recoil of Fully Reconstructed $B$ Meson Decays, Ph.D. Thesis, Royal Holloway, University of London (2005).

[91] H.A. Tanaka, Radiative Decays of the $B$ Meson, Ph.D. Thesis, SLAC-R-604 (2002).

[92] Charged kaon samples are taken from the decay chain $D^{*} \rightarrow D^{0} \pi_{\text {soft }}, D^{0} \rightarrow K \pi$. G. Mancinelli, Control Samples of Pions and Kaons and Kaon ID, presented at the BABAR Physics/Analysis Meeting, 1999, Padova, Italy, http://www.slac.stanford.edu/BFROOT/www/Physics/Tools/Pid/Meetings/ Padova/mancinelli.ps

[93] The default BABAR PID-killing look-up tables for pions in software release-10 are based on $D^{*}$ control samples. These have been replaced with tables generated from kinematically selected 3-1 prong $\tau^{+} \tau^{-}$events, after private communication with D. Aston. and T. Moore (2004).

M. Serra, Pion Sample from $\tau$-decay for PID studies, presented at the BABAR Physics/Analysis Meeting, 1999, Padova, Italy, http://www.slac.stanford.edu/ BFROOT/www/Physics/Tools/Pid/Control/PiK/taus/padovaM.pdf

[94] J. Beringer et al., B Tagging in BABAR: Status for the $2001 \sin 2 \beta$ Publications, BABAR Internal Analysis Document 119 (2002).

[95] A. Bukin et al., Measurement of the inclusive electron spectrum in charmless semileptonic $B$ decays near the kinematic endpoint, $B A B A R$ Internal Analysis Document 755 version 12 (2004).

[96] D. Fortin et al., Mesurement of $\left|V_{u b}\right|$ from inclusive semileptonic $B$ decays using $E_{l}^{*}$ and $q^{2}, B A B A R$ Internal Analysis Document 664 (2004).

[97] BABAR Collaboration, B. Aubert et al., Measurement of the $B \rightarrow D^{*}$ Form Factors in the Semileptonic Decay $\bar{B}^{0} \rightarrow D^{*+} \ell^{-} \bar{\nu}$, submitted to $32^{\text {nd }}$ International Conference on High-Energy Physics, 2004, Beijing, China, hep-ex/0409047. 
[98] CLEO Collaboration, J.E. Duboscq et al., Phys. Rev. Lett. 76, 3898 (1996).

[99] V. Lüth, private communication (2004).

[100] BABAR Collaboration, B. Aubert et al., Phys. Rev. Lett. 92, 071802 (2004).

[101] D. Bard et al., $b \rightarrow s \gamma$ branching fraction and energy spectrum measurement in RUN1 + RUN2 using the sum of exclusive modes, BABAR Internal Analysis Document 768 version 5 (2004).

[102] Starting with the definition for the convolution, it is straightforward to show that $\left\langle E\left(f_{t h} \otimes R\right)\right\rangle=\left\langle E\left(f_{t h}\right)\right\rangle+\langle E(R)\rangle$ and likewise for the variances.

E. W. Weisstein, CRC Concise Encyclopedia of Mathematics, 2nd. edition, CRC Press, 2002.

[103] H. Flächer, private communication (2005); O. Buchmüller and H. Flächer, hep-ph/0507253.

The same fitting technique has also been used in Reference [107].

[104] CLEO Collaboration, S. Chen et al., Phys. Rev. Lett. 87, 251807 (2001).

[105] Belle Collaboration, P. Koppenburg et al., Phys. Rev. Lett. 93, 061803 (2004)

[106] BABARCollaboration, B. Aubert et al., Phys. Rev. D 72, 052004 (2005).

[107] BABAR Collaboration, B. Aubert et al., Phys. Rev. Lett. 93, 011803 (2004).

[108] Type-II two-Higgs-doublet model proposes two Higgs doublet fields, one responsible for generating the masses of up-type quarks and the other for the masses of down-type quarks and charged leptons [3]. The constraint obtained from the $B \rightarrow X_{s} \gamma$ measurement is constant for $\tan \beta \gtrsim 1$, where $\tan \beta$ is the ratio of the vacuum expectation values of the two Higgs fields. Constraints for $\tan \beta \lesssim 1$ are stronger, but this region is disfavored by theory.

The computation for the actual mass limit has been performed using a Monte Carlo simulation program provided by R. Faccini in private communication (2005). The program is based on calculations of P. Gambino and M. Misiak, 
Nucl. Phys. B 611, 338 (2001). The additional 10\% theoretical uncertainty recently described in M. Neubert, Euro. Phys. J. 40, 165 (2005), has not been taken into account.

[109] N. Uraltsev, Phys. Lett. B 545, 337 (2002).

[110] D. Benson, private communications with the BABAR radiative penguin group (2005).

[111] H. Flächer and O. Buchmüller, An EMC Cluster Energy Calibration for Photons using VCS Events, 2004, unpublished; H. Flächer, private communication (2005).

[112] V.B. Golubev, V.N. Ivanchenko, Virtual Compton Scattering at BABAR, BABAR Internal Analysis Document 10 (2000). 